\title{
Experimental and Numerical Study of Inhaler Spray Characterization (Size Distributions and Velocity)
}

\author{
by \\ Abubaker Alatrash \\ A Thesis submitted to the \\ Faculty of Graduate Studies and Research \\ in partial fulfillment of the requirements for the degree of \\ Doctor of Philosophy \\ in
}

Mechanical Engineering

Ottawa-Carleton Institute for Mechanical and Aerospace Engineering

Department of Mechanical Engineering

Carleton University,

Ottawa, Ontario, Canada

(C) Copyright

Abubaker Alatrash , 2018 


\begin{abstract}
Particle size and velocity are two of the most significant factors that impact the deposition of pressurized metered-dose inhaler (pMDI) sprays in the mouth cavity. pMDIs are prominently used around the world in the treatment of patients suffering from a variety of lung diseases such as asthma and chronic obstructive pulmonary disease. Moreover, Soft Mist Inhalers (SMIs) are a new-generation of propellant free Inhalers. The first delivery inhalation spray system of this kind is the Spiriva Respimat inhaler, which has its advantages, such as better medication delivery to the lungs.
\end{abstract}

In the current study, particle velocity and size distribution are measured at three different locations along the centerline of the pMDI and the SMI spray using Phase Doppler Anemometry (PDA). pMDIs from four different pharmaceutical companies are tested, each using salbutamol sulfate as the medication. The SMI is tested using tiotropium bromide monohydrate as the active medication.

Measurements along the pMDI centerline (at 0, 75, and $100 \mathrm{~mm}$ downstream of the inhaler mouthpiece) showed that the spray velocities were bimodal in time for all four pMDI brands. Whereas, the velocity is almost constant for the SMI. Three probability density functions (PDFs) is tested, and the Rosin-Rammler PDF best fit the empirical spray size distribution data for all tested pMDIs. While log-normal PDF best fit the empirical data for SMI.

Particle size distribution for the Spiriva Respimat inhaler using the Anderson Cascade Impactor (ACI) is also assessed. The results indicate that a high percentage of inhaled medication was delivered to the lungs approximately $48 \%$, which is still quite significant when compared to common pMDI (about 8 to $20 \%$ ). 
Computational fluid dynamics, implementing mean-flow and turbulent tracking of particles, is used to determine the ability of the software ANSYS CFX package, to emulate the Ventolin HFA spray behavior. The results exhibit very good agreement between both numerical and experimental results. 


\section{Acknowledgments}

The first note of appreciation goes to my thesis supervisor Professor Matida for all his expert guidance, insight and valuable professional advice he has given me throughout my Ph.D. program. It has been a unique experience and a pleasure to work with him.

I would like to express my thanks and appreciation to Dr. Rym Mehri and Kenny Lee Slew for their help and support on the set-up and ACI system tests. Also, special thanks to the technical staff of the machine shop at the Mechanical and Aerospace Engineering Department at Carleton University who have aided with the construction of various testing rigs.

My appreciation and gratitude go to the other friends of the 6th floor Canal Building students, especially Dr. Rajab Legnain, Doma Hilewit, Frédéric Laforge, Phillip Drouillard, Dr. Tamer Beitelmal and Osama Hmood for sharing their conversation and ideas while I have been here, and providing a peaceful and enjoyable working environment

This thesis is dedicated to my family members who have always been there for me, specially my parents who sowed in me solid values which I have, together with my wife, brothers and sisters. Also, to the Alatrash family for their unconditional affection, encouragement, and support. 


\section{Contents}

List of Acronyms xiii

List of Symbols $\quad$ Xv

List of Symbols $\quad$ xvi

1 Background and Literature Review 1

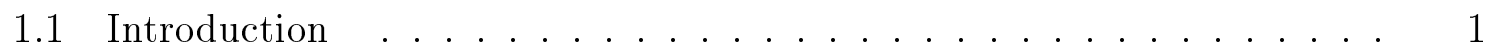

1.1.1 Thesis Statement ....................... 2

1.2 Pressurized Metered-Dose Inhaler $(\mathrm{pMDI}) \ldots \ldots \ldots$

1.2.1 pMDI Accessory Devices ............... 8

1.2.1.1 Add-On Devices . . . . . . . . . . 8

1.3 Soft Mist Inhalers (SMIs) . . . . . . . . . . . . 11

1.4 Characterization of pMDI Spray Velocities . . . . . . . . . . . 12

1.4.1 Experimental study ..................... 12

1.4 .2 Numerical Studies. . . . . . . . . . . . . . 19

2 Methods of Aerosol Particle Size Characterisation 24

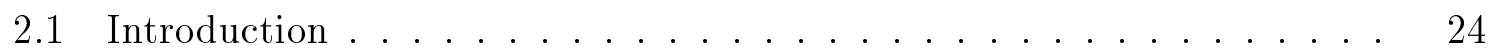

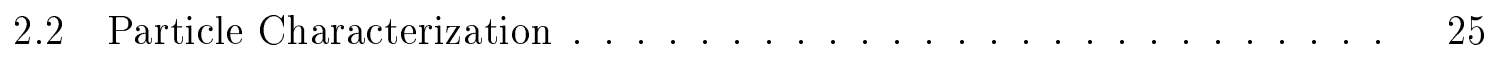

2.3 Andersen Cascade Impactor $(\mathrm{ACI}) \quad \ldots \ldots \ldots$. . . . . . . . 28 
2.4 Theory of Laser and Phase Doppler Anemometry (PDA) . . . . . . . 29

2.4.1 The Measurement Volume .............. 30

2.5 Laser Doppler Anemometry . . . . . . . . . . . . . . . . 30

2.5.1 Directional Ambiguity ............... 34

2.6 Phase Doppler Anemometry $(\mathrm{PDA}) \ldots \ldots . \ldots . \ldots . \ldots$

2.6.1 Principles of operation . . . . . . . . . . . 35

2.6.2 Light-scattering by particles .............. 36

2.6.3 Optical parameters of a Phase Doppler Measurement System . . 37

3 Experimental Setup and Procedure $\quad 42$

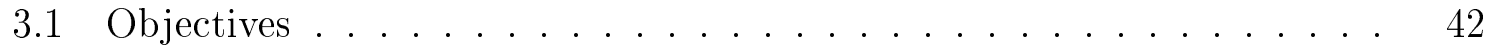

3.2 Characterization of Aerosol Velocity and Size Distribution by PDA . . 43

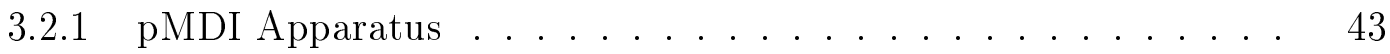

3.2.1.1 Custom Schmitt Trigger . . . . . . . . . 45

3.2.2 Spiriva Respimat Apparatus . . . . . . . . . . . 47

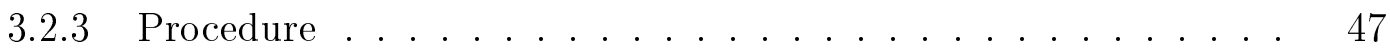

3.2.4 Summary of Process Diagram . . . . . . . . . . . 52

3.2.5 Selection of Instrument Parameters . . . . . . . . . . . . 54

3.2.5.1 Laser Power ................. 54

3.2.5.2 High Voltage (HV), and Signal to Noise Ratio (SNR) . 55

3.2.5.3 Transmitter Optic Aperture .......... 55

3.2.5.4 Receiver Optic Aperture ............ 56

3.2.5.5 Processor Limitations _........... 58

3.2.5.6 Parameter Selection Summary.......... 61

3.3 Spiriva Respimat Aerosol characterization using ACI (Andersen Cascade Impactor $\ldots \ldots \ldots$. . . . . . . . . . . . . 61

3.3.1 Experimental Apparatus ................... 61 
3.3.2 Experimental Procedure . . . . . . . . . . . . . . 63

3.3.3 Absorbance Measurement . . . . . . . . . . . . . . 63

3.4 CCD High Speed Camera _. . . . . . . . . . . . . . . 64

4 Numerical methods $\quad 66$

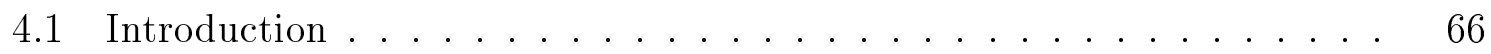

4.2 Geometry . . . . . . . . . . . . . . . . . . 66

4.3 Boundary conditions $\ldots \ldots \ldots \ldots \ldots \ldots \ldots \ldots$

4.4 Drug properties and spray characterisation . . . . . . . . . 68

4.5 Single phase $($ air) flow $\ldots \ldots \ldots$. . . . . . . . . . . 69

4.6 Lagrangian particle tracking (particulate phase) . . . . . . . . . 73

4.7 Grid Convergence studies . . . . . . . . . . . . . . . . 76

4.7.1 Grid and Domain Convergence Studies Results . . . . . . . 78

5 Results and Discussions $\quad 82$

5.1 Introduction . . . . . . . . . . . . . . . 82

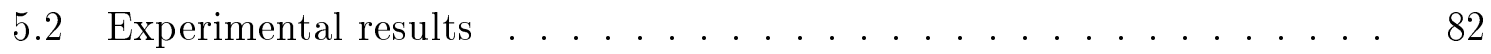

5.2 .1 Temporal analysis of inhalers spray . . . . . . . . . . 82

5.2.1.1 Velocity analysis of the inhaler spray . . . . . . 83

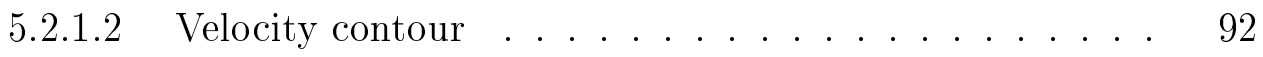

5.2.1.3 Particle diameter analysis of the inhaler spray . . . . 94

5.2 .2 Spatial analysis of the inhaler spray $\ldots \ldots \ldots \ldots$

5.2.2.1 Velocity analysis of the pMDI spray _. . . . . . . 103

5.2.2.2 Particle diameter analysis of the inhaler spray . . . . . 107

5.2 .3 Particle size distributions . . . . . . . . . . . . . . . 113

5.2.4 Aerosol Characterization using ACI . . . . . . . . . . 120

5.2.4.1 Data and Statistical Analyses . . . . . . . . . . 120

5.2.4.2 Particle size distribution . . . . . . . . . . . . . . 121 
5.2.4.3 Aerosol deposition . . . . . . . . . . . . . . . . . 124

5.2 .5 Error and Uncertainty . . . . . . . . . . . . . 125

5.2.5.1 Errors in measurements for PDA system . . . . . . 127

5.2.5.2 Errors in measurements for particle size and deposition using ACI . . . . . . . . . . . . . . . . . . . 129

5.3 High Speed Camera results . . . . . . . . . . . . . . . 130

5.3.1 Spray Distance and Duration for Spiriva Respimat inhaler . . . 130

5.3 .2 Respimat SMI versus pMDI . . . . . . . . . . . . . 130

5.4 Numerical results . . . . . . . . . . . . . . . . . . . . 133

6 Conclusions and Recommendations 139

6.1 Summary of results . . . . . . . . . . . . . . . . . . 139

6.2 Summary of contributions . . . . . . . . . . . . . . 142

6.3 Recommendations for future work . . . . . . . . . . . . . . 143

7 Uncertainty in measurments $\quad 159$

7.1 Errors in measurements for PDA system . . . . . . . . . . . . 159

7.2 Errors in measurements for particle size and deposition using ACI . . 163 


\section{List of Figures}

1.1 Photographs of medical devices for drug delivery. . . . . . . . . . 3

1.2 Components and internal structure of pMDI. . . . . . . . . . 6

1.3 Photographs of Add-On devices (Spacers). . . . . . . . . . . . . 9

1.4 Add-on devices: ODAPT soft mist adapter (left) and EcoMask facemask with ODAPT adapter (right). . . . . . . . . . . . . 10

1.5 The Respimat softmist inhaler. . . . . . . . . . . . . . . . 11

1.6 Droplet velocity in the center of the mouthpiece exit plane. Adapted

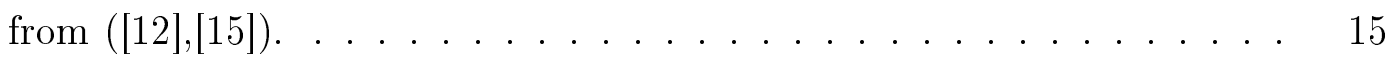

1.7 Temporal evolution of the spray velocity as emitted from a Ventolinbrand pMDI. Adapted from $[7] . \ldots \ldots \ldots \ldots$

1.8 The velocity vector flow-field and selected radial (vertical) profiles of axial velocity for three-time delay actuations into the quiescent air. adapted

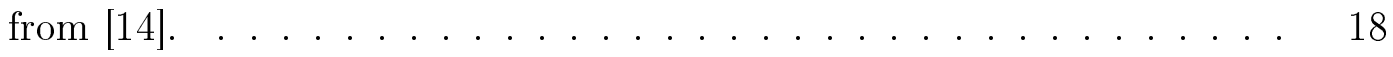

1.9 Schematic of the three factors of the pMDI actuator orifice. adapted from $[44] . \ldots \ldots \ldots \ldots \ldots \ldots$

2.1 Schematic diagram of Cascade Impactor.Adapted from [64] . . . . . 26

2.2 Inertial impaction of particles and airflow in ACI. . . . . . . . . . 26

2.3 ACI stage, consisting of sieve (left) and the respective collection plate

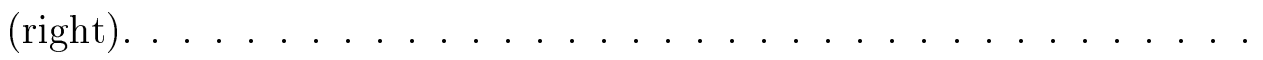


2.4 Assembled Andersen Cascade Impactor. . . . . . . . . . . . . . . . 29

2.5 Measurement volume from intersecting coherent laser beams. . . . . . . 31

2.6 The formation of the measurement volume. Adapted from [72] . . . . . 31

2.7 Block diagram of Laser Doppler Anemometry system. Adapted from [72]. 32

2.8 Modern two beam LDA configuration. Adapted from [79]. . . . . . . . 34

2.9 Light scattering by droplet of water, where $n_{r e l}=1.33$ and $d_{p} \gg \lambda_{b}$. Adapted from $[83] . \ldots \ldots \ldots \ldots$. . . . . . . . . . . . . 37

2.10 Optical arrangement of a two detector PDA system. Adapted from [81]. 38

2.11 The Optical parameters of a PDA set-up. Adapted from [81] . . . . . . 39

2.12 The phase shift between two detectors and the $2 \pi$ ambiguity. Adapted

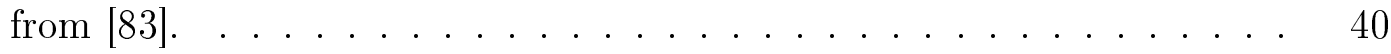

2.13 Modern PDA configuration. Adapted from [79]. . . . . . . . . . . . 41

2.14 Extension of the diameter range in a three-detector PDA system. Adapted from $[83] . \ldots \ldots \ldots \ldots \ldots \ldots \ldots$

3.1 Actuation mechanism of the pMDI and the mounting apparatus. . . . 44

3.2 Photograph of the moulded replica of the pMDI casing. . . . . . . . 45

3.3 Schmitt trigger and monostable vibrator circuit. . . . . . . . . . 46

3.4 The mounting apparatus of the Spiriva Respimat. . . . . . . . . . 48

3.5 Experimental measurement locations. . . . . . . . . . . . . 50

3.6 PDA system and experimental apparatus. . . . . . . . . . . 51

3.7 Experimental process diagram. . . . . . . . . . . . . 53

3.8 Droplet diameter dependency on incident laser power. . . . . . . . . . 54

3.9 Droplet velocity dependency on incident laser power. . . . . . . . . 55

3.10 The mechanism of the slit aperture for Gaussian intensity distributed measurement volumes. Adapted from [78]. . . . . . . . . . . 57

3.11 Data coupling analysis. . . . . . . . . . . . . . . . . . 59 
3.12 Schematic of the experimental setup used for Repimat. . . . . . . . . . 62

3.13 Schematic of high-speed camera setup. . . . . . . . . . 65

4.1 The testbox representation. . . . . . . . . . . . 67

4.2 Geometry made in ICEM by ANSYS and the mesh intensity. . . . . . . 68

4.3 Areas of interest for grid convergence studies. . . . . . . . . . 78

4.4 Velocity along the $\mathrm{x}$-axis for tested geometries. . . . . . . . . . 80

5.1 Experimental measurement locations. . . . . . . . . . . . 84

5.2 Raw data for the particle velocities for five actuations for each inhaler at $x=0 \mathrm{~mm} \ldots \ldots \ldots \ldots \ldots \ldots$

5.3 Raw data for the particle velocities for five actuations for each inhaler at $x=75 \mathrm{~mm} \ldots \ldots \ldots \ldots \ldots$

5.4 Raw data for the particle velocities for five actuations for each inhaler at $x=100 \mathrm{~mm} \ldots \ldots \ldots \ldots \ldots \ldots$

5.5 Temporal evolution of the measured spray velocity for each inhaler at

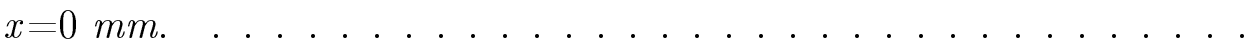

5.6 Raw data for the particle velocities for five actuations for Spiriva Respi-

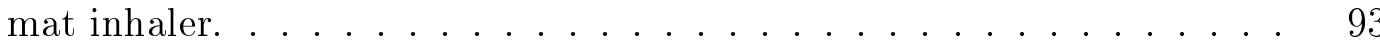

5.7 Ventolin HFA inhaler velocity contour plot from PDA measurement at $x=0 \mathrm{~mm} \ldots \ldots \ldots \ldots \ldots \ldots$

5.8 Novo-Salbutamol HFA inhaler velocity contour plot from PDA measurement at $x=0 \mathrm{~mm} \ldots \ldots \ldots \ldots \ldots$

5.9 Airomir HFA inhaler velocity contour plot from PDA measurement at $x=0 \mathrm{~mm} \ldots \ldots \ldots \ldots \ldots \ldots$

5.10 Apo-Salvent CFC Free inhaler velocity contour plot from PDA measurement at $x=0 \mathrm{~mm} \ldots \ldots \ldots \ldots \ldots$ 
5.11 Raw data for the particle size for five actuations for each inhaler at $x=0$

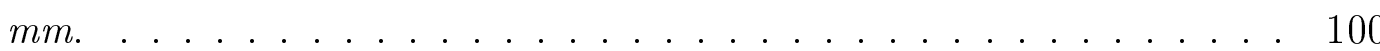

5.12 Raw data for the particle size for five actuations for each inhaler at $x=75$ $m m \ldots \ldots \ldots \ldots \ldots \ldots \ldots 10 \ldots \ldots \ldots \ldots \ldots \ldots \ldots$

5.13 Raw data for the particle size for five actuations for each inhaler at $x=100$

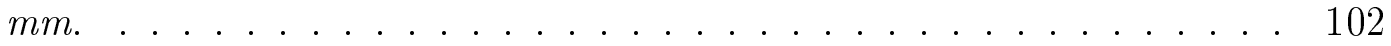

5.14 Raw data for the particle size for five actuations for Spiriva Respimat inhaler. . . . . . . . . . . . . . . . . 104

5.15 Velocity spatial analysis for pMDI inhalers. . . . . . . . . . 106

5.16 Velocity spatial analysis for Spiriva Respimat inhaler. . . . . . . . . 108

$5.17 D_{10}$ profiles for particles diameter spatial analysis. . . . . . . . . . 111

$5.18 D_{30}$, and $D_{\text {v50 }}$ profiles for particles diameter spatial analysis. . . . . . 112

5.19 Particle diameter spatial analysis for Spiriva Respimat inhaler. . . . . . 113

5.20 Histogram of the experimental data of particles diameter with curve fitting of probability distribution functions at $0(\mathrm{~mm}) \ldots \ldots . \ldots 116$

5.21 The particles diameter distributions of six time intervals for each inhaler

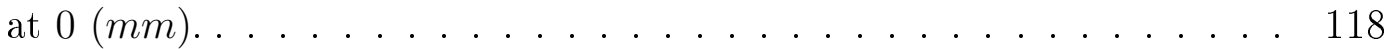

5.22 Histogram of the experimental data of particles diameter with curve fitting of PDFs for Spiriva Respimat inhaler. . . . . . . . . . . . 119

5.23 The particles diameter log-normal PDF of six-time intervals for Spiriva

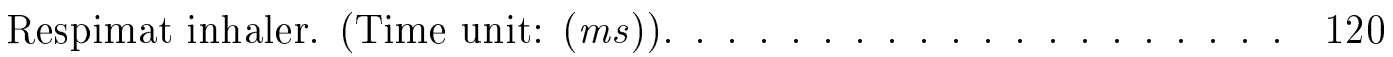

5.24 Aerodynamic particle size distribution under normal and humid conditions.122

5.25 Aerodynamic particle size distribution for both locations $75 \mathrm{~mm}$ and 100

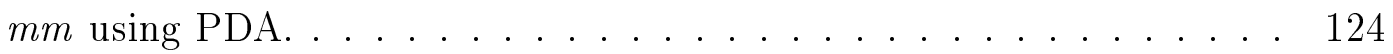

5.26 Percent deposition of medication at two relative humidity levels of (a)

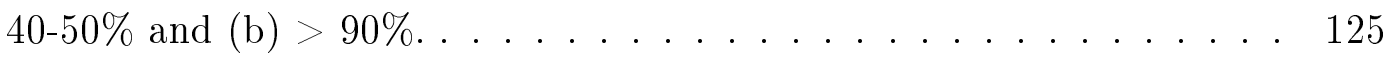

5.27 Distance travelled by the spray as a function of time. . . . . . . . . . 131 
5.28 Spray characteristics of Respimat SMI versus Ventolin HFA pMDI. . . 134

5.29 Velocity magnitude contours of the air in the domain. . . . . . . . . 135

5.30 Velocity magnitude contours of the particle in the domain. . . . . . . 137

5.31 Spray velocity comparison numerical against experimental. . . . . . . 138 


\section{List of Tables}

3.1 Maximum droplet size vs mask selection. . . . . . . . . . . 58

3.2 Summary of instrument parameter selection. . . . . . . . . . 60

3.3 Controlled parameters used in the study. . . . . . . . . . . 61

4.1 Parameters used in the simulation. . . . . . . . . . . . . . 69

4.2 Coefficients of the SST model. . . . . . . . . . . . . . . . . 73

4.3 Testboxes dimension.. . . . . . . . . . . . . . . . 77

5.1 Mean particle velocity measured at 0,75 , and $100 \mathrm{~mm}$ away from the origin along the centerline. (Units: $m / s$ ). . . . . . . . . . 105

5.2 Mean particle velocity measured at $-18.5,0,75$, and $100 \mathrm{~mm}$ away from the origin along the centerline Spiriva Respimat inhaler. (Units: $\mathrm{m} / \mathrm{s}$ ). 107

5.3 Particle size measurement for the four tested MDI inhalers $D_{10}, D_{30}$, and $D_{\mathrm{v} 50}:($ Units $\mu m) . \ldots \ldots \ldots \ldots \ldots \ldots$

5.4 Particle size measurement for the four Spiriva Respimat inhaler $D_{10}, D_{30}$, and $D_{\mathrm{v} 50}:($ Units $\mu m) \ldots \ldots \ldots \ldots \ldots \ldots \ldots \ldots \ldots \ldots \ldots \ldots \ldots \ldots$

5.5 Optimum parameters for three selected PDFs for tested pMDIs. . . . . 115

5.6 Optimum parameters for three selected PDFs for Spiriva Respimat inhaler.119

5.7 Particle size distributions of aerosol from Spiriva Respimat inhaler under normal and humid conditions. . . . . . . . . . . . . . . . 122

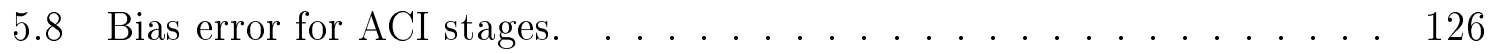


5.9 Measurement errors for particle size using PDA system for tested inhalers at $x=0(\mathrm{~mm})$ at $95 \%$ level of confidence. . . . . . . . . 128

5.10 Measurement errors for particle velocity using PDA system for tested inhalers at $x=0(\mathrm{~mm})$ at $95 \%$ level of confidence. . . . . . . . 129

5.11 Measurement errors for particle size and velocity using PDA system for tested inhalers at $x=0(\mathrm{~mm})$ at $95 \%$ level of confidence. . . . . . .

5.12 Measurement errors for particle size using ACI system under normal humidity $(40-50 \% \mathrm{RH})$ at $95 \%$ level of confidence. . . . . . . . . .

5.13 Measurement errors for deposition on ACI system under normal humidity $(40-50 \% \mathrm{RH})$ at $95 \%$ level of confidence . . . . . . . . . 132

7.1 Measurement errors for particle size using PDA system for tested inhalers $75(\mathrm{~mm})$ at $95 \%$ level of confidence. . . . . . . . . . . . .

7.2 Measurement errors for particle size using PDA system for tested inhalers $100(\mathrm{~mm})$ at $95 \%$ level of confidence. . . . . . . . . . 160

7.3 Measurement errors for particle velocity using PDA system for tested inhalers $75(\mathrm{~mm})$ at $95 \%$ level of confidence. . . . . . . . . . .

7.4 Measurement errors for particle velocity using PDA system for tested inhalers $100(\mathrm{~mm})$ at $95 \%$ level of confidence. . . . . . . . . . . .

7.5 Measurement errors for particle diameter using PDA system for tested Spiriva Respimat inhaler at $95 \%$ level of confidence. . . . . . . . . . . . 161

7.6 Measurement errors for particle velocity using PDA system for tested Spiriva Respimat inhaler at $95 \%$ level of confidence. . . . . . . . . . . . 162

7.7 Measurement errors for particle size using ACI system under $>90 \% \mathrm{RH}$ at $95 \%$ level of confidence. . . . . . . . . . . . . . . .

7.8 Measurement errors for deposition on ACI system under $>90 \% \mathrm{RH}$ at $95 \%$ level of confidence. . . . . . . . . . . . . . . 164 


\section{List of Acronyms}

\begin{tabular}{ll}
\hline \hline Acronym & Definition \\
\hline MDI & Metered dose inhaler \\
pMDI & Pressurized metered dose inhaler \\
SMI & Soft mist inhalers \\
DPI & Dry powder inhalers \\
COPD & Chronic obstructive pulmonary disease \\
CFC & Chlorouorocarbon \\
HFA & Hydrofluoroalkane \\
VHC & Valve holding chamber \\
DF & Phase Doppler Anemometry \\
PDA & Deposition fraction \\
LDA & Laser Doppler Anemometry \\
ACI & Anderson Cascade Impactor \\
PIV & Particle Image Velocimetry \\
VNA & Vortex nozzle actuator \\
MT & Mouth-throat \\
CAG & Capillary aerosol generation \\
LLS & Laser Light Scattering \\
SLPM & Standard liters per minute \\
CFD & Computational fluid dynamics \\
IP & Induction port \\
TTL & Transistor-Transistor Logic \\
BNC & Bayonet Neill-Concelman Connector \\
HV & High voltage \\
SNR & Signal to noise ratio \\
RH & Relative humidity \\
URANS & Unsteady Reynolds-Averaged Navier- Stokes \\
SST & Shear Stress Transport \\
LES & Large Eddy-simulation \\
PDF & Probability Density Functions \\
SD & Ptandard deviation \\
PSD &
\end{tabular}




\begin{tabular}{ll}
\hline \hline Acronym & Definition \\
\hline MMAD & Mass median aerodynamic diameter \\
GSD & Geometric standard deviation \\
UV & Ultra violet \\
CFL & Courant number \\
fps & Frame per second
\end{tabular}

xiv 


\section{List of Symbols}

\begin{tabular}{|c|c|c|}
\hline Symbol & Description & Units \\
\hline$D_{10}$ & Mean diameter & $\mu m$ \\
\hline$D_{30}$ & Volume mean diameter & $\mu m$ \\
\hline$D_{V 50}$ & Volume median diameter & $\mu m$ \\
\hline$f_{r}$ & Incident beam velocity & $m / s$ \\
\hline$f_{D}$ & Doppler frequency & $H z$ \\
\hline$f_{o}$ & Bragg cell shifted frequency & $H z$ \\
\hline$n_{\text {rel }}$ & Relative refraction index & - \\
\hline$V_{p}$ & Particle velocity & $\mathrm{m} / \mathrm{s}$ \\
\hline$x$ & Axial distance from pMDI tip & $m m$ \\
\hline$r$ & Radial distance from pMDI axis & $m m$ \\
\hline$d_{p}$ & Particle diameter & $\mu m$ \\
\hline$R_{e x t}$ & External timing resistance & $K \Omega$ \\
\hline$C_{\text {ext }}$ & External timing capacitor & $p F$ \\
\hline$t_{w}$ & Output pulse width & $n s$ \\
\hline$t$ & Time & $s$ \\
\hline$P$ & Time-averaged pressure & $P a$ \\
\hline g & Gravitational acceleration & $m / s^{2}$ \\
\hline$R e_{p}$ & Particle Reynolds number & - \\
\hline$\triangle t$ & Time step & $s$ \\
\hline$\triangle x$ & Element size & $m$ \\
\hline Directional & Definition & Units \\
\hline $\mathrm{U}, \mathrm{V}, \mathrm{W}$ & Directional velocities & $\mathrm{m} / \mathrm{s}$ \\
\hline $\mathrm{u}, \mathrm{v}, \mathrm{w}$ & Instantaneous directional velocities & $\mathrm{m} / \mathrm{s}$ \\
\hline $\mathrm{x}, \mathrm{y}, \mathrm{z}$ & Direction along axis & $m$ \\
\hline $\mathrm{i}, \mathrm{j}, \mathrm{k}$ & Directional subscripts & - \\
\hline
\end{tabular}




\begin{tabular}{lll}
\hline Greek symbols & Definition & Units \\
\hline$\lambda_{b}$ & Incident beam wavelength & $\mathrm{nm}$ \\
$\phi$ & Beam intersection angle & $\mathrm{rad}$ \\
$\theta$ & Scattering Angle & $\mathrm{rad}$ \\
$\psi$ & Inclination Angle & $\mathrm{rad}$ \\
$\Phi$ & Phase difference & $\mathrm{rad}$ \\
$\beta$ & Detector geometrical factor & - \\
$\mu$ & Turbulent viscosity & $\mathrm{m}^{2} / \mathrm{s}^{2}$ \\
$\alpha$ & Angle of spray & $\mathrm{degrees}$ \\
$\sigma_{3}$ & SST Constant & - \\
$\alpha_{3}$ & SST Constant & - \\
$\beta_{3}$ & SST Constant & - \\
$\rho$ & Density & $\mathrm{kg} / \mathrm{m}^{3}$ \\
$\delta_{i j}$ & Kronecker delta & - \\
$k$ & Turbulent kinetic energy & $\mathrm{m}^{2} / \mathrm{s}^{2}$ \\
$\varepsilon$ & Dissipation & - \\
$\nu$ & Viscosity & $\mathrm{m}^{2} / \mathrm{s}$ \\
$\varpi$ & Turbulent frequency & $1 / \mathrm{s}$
\end{tabular}




\section{Chapter 1}

\section{Background and Literature Review}

\subsection{Introduction}

Respiratory illnesses such as asthma, chronic obstructive pulmonary disease (COPD), tuberculosis (TB), and cystic fibrosis are all debilitating diseases. It was estimated that over 3.0 million Canadians were touched by these illnesses in 2007. In addition, other respiratory diseases like influenza, pneumonia, bronchiolitis, respiratory distress syndrome and sleep apnea also influence children and seniors [1]. The Canadian Lung Association stated that $10 \%$ of people may have a respiratory illness requiring treatment at a certain stage of their lives. Also, it was reported in 2011 by the Public Health Agency of Canada that there were more than 2.4 million (8.4\%) canadians, aged 12 years and over, who were living with asthma between the years 2009 and 2010 [2]. In addition, it's being reported by Canadian Health Measures Survey (CHMS) that $12 \%$ of Canadians 35 to 79 years of age had a measured airflow obstruction consistent with COPD in the years between 2012 to 2015 [3].

Well known techniques for delivering aerosol medicines to patients include the use of pressurized metered-dose inhalers (pMDIs), soft mist inhalers (SMIs), dry powder inhalers (DPIs) and nebulizers (Figure 1.1). These are frequently recommended for treat- 
ing traditional lung diseases, particularly asthma and chronic obstructive pulmonary disease (COPD) [4]. Such devices are of high interest when it comes to the delivery of medication, using the lungs as a gateway [5].

Dry powder inhalers (DPIs) are becoming a widespread substitution to traditional delivery devices used for asthma. These kind of devices depend only on the force of the inhalation for delivering the medication to the lungs [6]. To use the device, patients must trigger a lever and then inhale from the mouthpiece. This process eliminates the requirement for coordination between actuation and inhalation.

Nebulizers are used by pumping air through a liquid medication. This process produces an aerosol consisting of a fine mist of medication droplets [7]. The aerosolized medication is inhaled and will deposit into the lung. The nebulizer may be used with a mouthpiece or a mask. To acquire the proper dosage of medication, the drug must deposit at the lower airways of the respiratory system [8]. Schuepp et al. [6] showed that the lung deposition of medication using nebulizers ranges from $0.5 \%$ to $12 \%$. Due to large particle sizes, the majority of the medication deposition is in the oropharynx and upper airways. Nebulizers do not require breathing coordination, as opposed to pMDIs. In this case, the patients only inhale and exhale naturally until the medication dosage is delivered.

This chapter will present the background information regarding currently used pMDI and SMI devices for delivering aerosolized medication to the lungs.

\subsubsection{Thesis Statement}

The goal of this thesis is to investigate and characterize both, experimentally and numerically the particle size distribution as well as the particle velocity at multiple locations downstream of pMDI and SMI. For this purpose, different inhalers are tested to investigate differences in particle size generation, distribution and velocity. In this case, four different commercially available pressurized Metered Dose Inhalers are tested, 


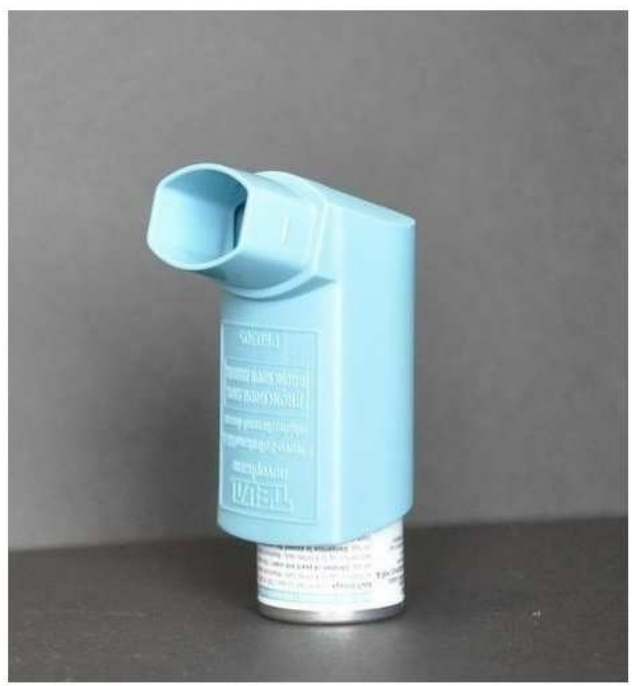

a)pMDI

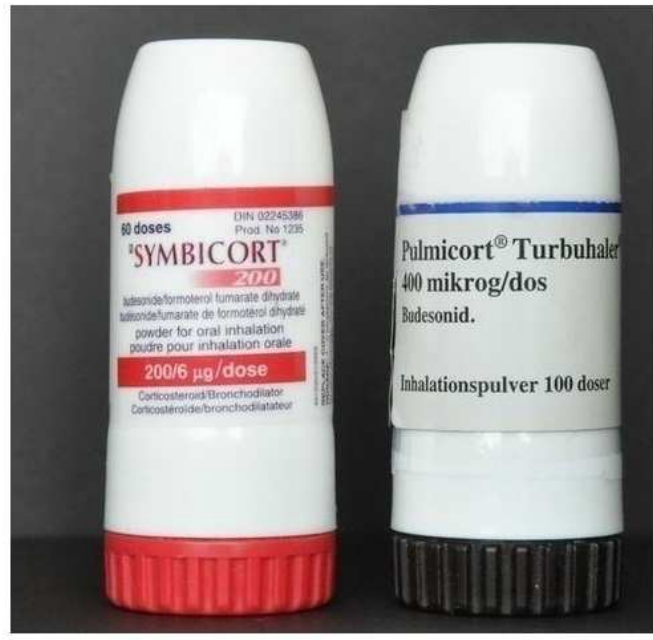

c) DPI

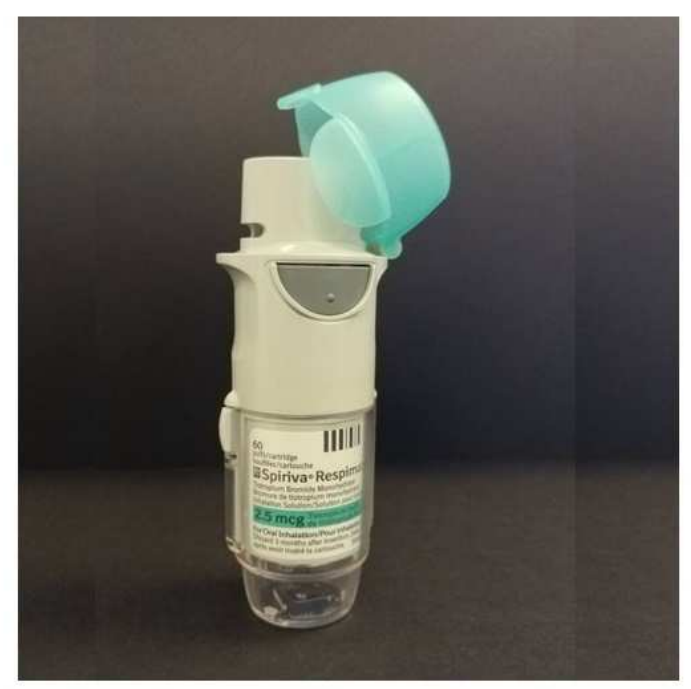

b) SMI

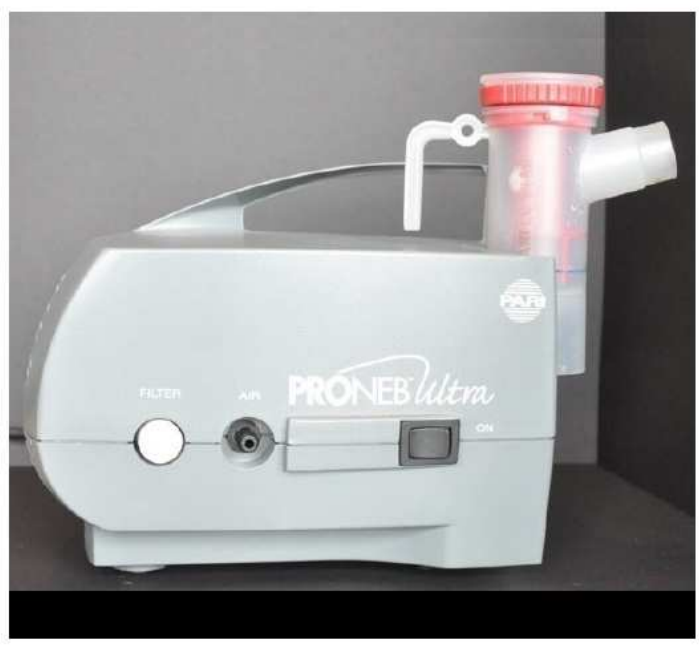

d) Nebulizer

Figure 1.1: Photographs of medical devices for drug delivery. 
namely: Apo-Salvent CFC Free, Airomir HFA, Novo- Salbutamol HFA, Ventolin HFA, and a new generation of Soft Mist Inhaler (Spiriva Respimat SMI). All pMDI inhalers are tested using the same medication (salbutamol sulfate with hydrofluoroalkane (HFA) based suspensions).

Experimentally, particle sizes and particle velocities are determined using Phase Doppler Anemometry (PDA). This system measures the particle velocities as they pass through the measurement volume using the theory of a differential Doppler process when an interaction occurs between (1) the intersection of two incident monochromatic laser beams (2) a moving particle. Particle size can also be measured using the principle of inertial impaction.

An Anderson Cascade Impactor (ACI) consists of a system of 8 functional stages that filters the particles based on their aerodynamic size, by causing the particles to impact and adhere to stage surfaces due to the specific velocity of the particle. Experimental data such as the particle size distribution and velocity are used as boundary conditions to numerically solve for the spray.The CFD (ANSYS CFX) simulations are performed by using the Unsteady Reynolds Averaged Navier Stokes equations (URANS), along with Lagrangian particle tracking and shear stress transport turbulence models to predict the pMDI behaviour.

With this study, the fine particle fraction (with a mass median diameter less than $5 \mu \mathrm{m})$ that is delivered to the patient and deposits in the lower airways can be determined and compared for the different inhalers being tested. Determining the fine particle fraction for the different types of the inhalers can help determine the medication losses in the patient mouth cavity and the oropharynx.

Flow and particle dynamics in pMDIs were extensively investigated in previous studies $([9-16])$. However, many patients complain about the medication's taste in their mouth after the inhalation, which is due to the deflection in the spray - a phenomenon that is not fully understood. Hence, in this study, particle size and velocity measure- 
ments are taken at several locations providing a more in depth analysis of the flow of pMDI inhalers. Consequently, a deep study is conducted to investigate the deflection in the spray for the tested pMDIs. A velocity contour on a cross-sectional area is taken at the edge of the mouthpiece and multiple locations on the vertical axis. The results are believed to be the first in the filed. In addition, the analysis on the particle distribution at several time intervals is carried out to investigate the particle size release with time which is a first in the area.

Secondly, particle size distribution for the Spiriva Respimat inhaler using ACI, for two levels of relative humidity are assessed. Thus, the aim of this study is to investigate the particle size distribution and the aerosol deposition to the lungs and compare it against the medication delivery by pMDI.

Finally, a numerical simulation is verified experimentally to predict experimental data obtained with the PDA thus, ensuring the proper implementation of the experimental data and assessing the capabilities of ANSYS CFX software to predict the pMDI spray behaviour in both single phase (air) and particulate domains. This work provides

a framework for the simulation of spray flows of different inhalers as well as for those who seek to improve current and future inhaler designs.

\section{$1.2 \quad$ Pressurized Metered-Dose Inhaler (pMDI)}

pMDI devices deliver a short burst of aerosolized medication, which is inhaled into the lungs. pMDIs have rapidly gained recognition since their introduction in the second half of the last century. Millions of patients around the world currently use pMDIs [17]. As a result of their effectiveness and simplicity of use, pMDIs are considered to be the most widely prescribed aerosol delivery system [18]. A pMDI (Figure 1.2) typically consists of three parts: the canister, the actuator and the metering valve [19]. It has a pressurized metal canister usually made from aluminum (including a medication and propellant) in a plastic sleeve with a mouthpiece. The propellants used are usually 
chlorofluorocarbons (CFCs) or hydrofluoroalkanes (HFAs). CFCs deplete the ozone layer and were associated with increased health risks, including cataracts, therefore, this substance has been prohibited according to the terms of an international agreement called the Montreal Protocol [8].

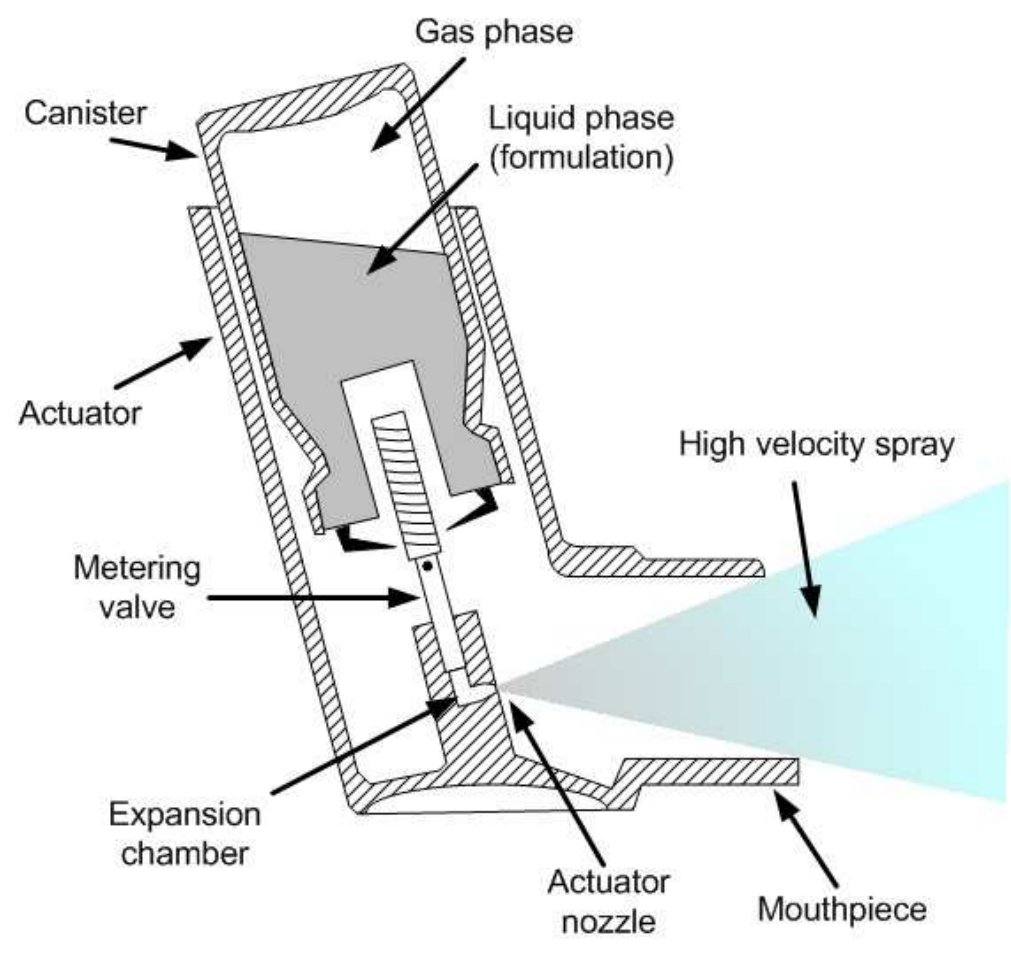

Figure 1.2: Components and internal structure of pMDI.

For some pMDI designs, the canister position is reversed, and then is appended to a plastic actuator. Therefore, controlled dose variations of aerosol can be generated when the canister is pressed into the actuator. Also, regarding the propellant many manufacturers employed an approach which consisted of using ethanol to enhance the solubility of the surfactants. Schering-Plough developed a formulation of salbutamol containing HFA-134a, oleic acid, and ethanol. Similarly, a formulation including beclomethasone dipropionate soluble in HFA-134a with ethanol was developed and provides a significantly smaller droplet size than that of CFC pMDI (mass median diameter of 1 versus $4 \mu m$, respectively) at a lower spray velocity. As a result of this technique, the lung 
deposition was increased by more than $50 \%$ of the inhaled dose. This formulation provides accurate quantification of the dose and prevents having to shake the inhaler prior to use, as is generally recommended for suspension formulations. Nevertheless, chemical disintegration is a major issue for drugs in solution. To reduce the evaporation of propellant, fluids like glycerol and polyethylene glycol, which are low-vapor-pressure liquids, are used in small quantities. Therefore, a different taste is generally found in new devices compared to old ones. This difference is a result of variations in the excipients, elastomers, and propellants. The spray discharged from the actuator of HFA pMDIs has a lower impact force and a smaller plume which leads to a softer and warmer feel inside the mouth of the patient [18].

The energy used for dispersion of the medication in these delivery systems is gained from the high vapor pressure propellant. pMDIs are inexpensive, simple to use, and effective. However, the pMDI spray releases large propellant droplets moving at a velocity of over $30 \mathrm{~m} / \mathrm{s}$, which instantly impact the oropharyngeal wall, which leads to about $80 \%$ losses in the emitted dose, as has been reported for a pMDI being used correctly [20], with only about 10-20\% of the drug deposited in the lungs [21]. pMDIs are adequate, ligh, portable, multi dose, and can be stored in any orientation without leakage, for the duration of the canister life; pMDIs dependably provide consistent dosing [22].

Despite all of the above advantages, pMDIs have some limitations such as inappropriate use of the device by numerous patients. Roughly $50 \%$ of adult patients and a higher percentage of children use pMDIs incorrectly, resulting in delivering little or no medication to the lungs [23]. Inconsistent dosing can be considered as an enormous limitation of the technology. This comprises the impact of hand-breath asynchrony, excessive inspiratory flow velocity, nose-breathing and the cold-Freon effect. The latter may happen in the event the cold aerosol plume reaches the oropharynx, thus the patient stops the inhalation [24,25]. 
Regardless of that, the current pMDI design stays a part of everyday life for many patients. As stated in previous collected data for regularly prescribed, short-acting bronchodilator devices; sales in 16 European countries in the period between 2002 and 2008 for retail pharmacy exhibited that pMDIs were the most prescribed devices to treat its related diseases [26]. In addition, through the last quarter of 2011, the participation of pMDIs in retail sales of inhalers was more than 55\% (some 25.4 million units) in the five largest European countries (France, Germany, Italy, Spain, and the UK), which was in comparison higher than non-refillable and refillable DPIs, and breath-actuated MDIs [27].

In terms of efficacy, the aerosol device must deliver medication to the lower respiratory tract. Consequently, particles emitted from the aerosol device must have a mass median aerodynamic diameter generally ranging from $0.5 \mu \mathrm{m}$ to $4.5 \mu \mathrm{m}$ for ensuring proper inhalation and deposition in the airways. Patients must inhale the aerosol with a deep and slow breath for optimal aerosol deposition in the airways. Good sedimentation of the medication particles requires the patient to breath-hold during the inhalation of the medicine [28].

\subsection{1 pMDI Accessory Devices}

Reasonably low inspiratory flow is required for effective deposition of aerosols from pMDIs, as well as deep inhalation and a breath hold. An infant's capability to efficiently use an aerosol device increases as he gets older. For patients with difficulty synchronizing actuation and breath, accessory devices can reduce mouth deposition and increase airway deposition. [24], [29], and [25].

\subsubsection{Add-On Devices}

Add-on devices include spacers and valved holding chambers (VHC). The former 
are devices adding additional volume for capturing aerosol from a pMDI by expanding the distance between the pMDI nozzle and the oropharynx, although coordination of actuation is needed with inhalation.

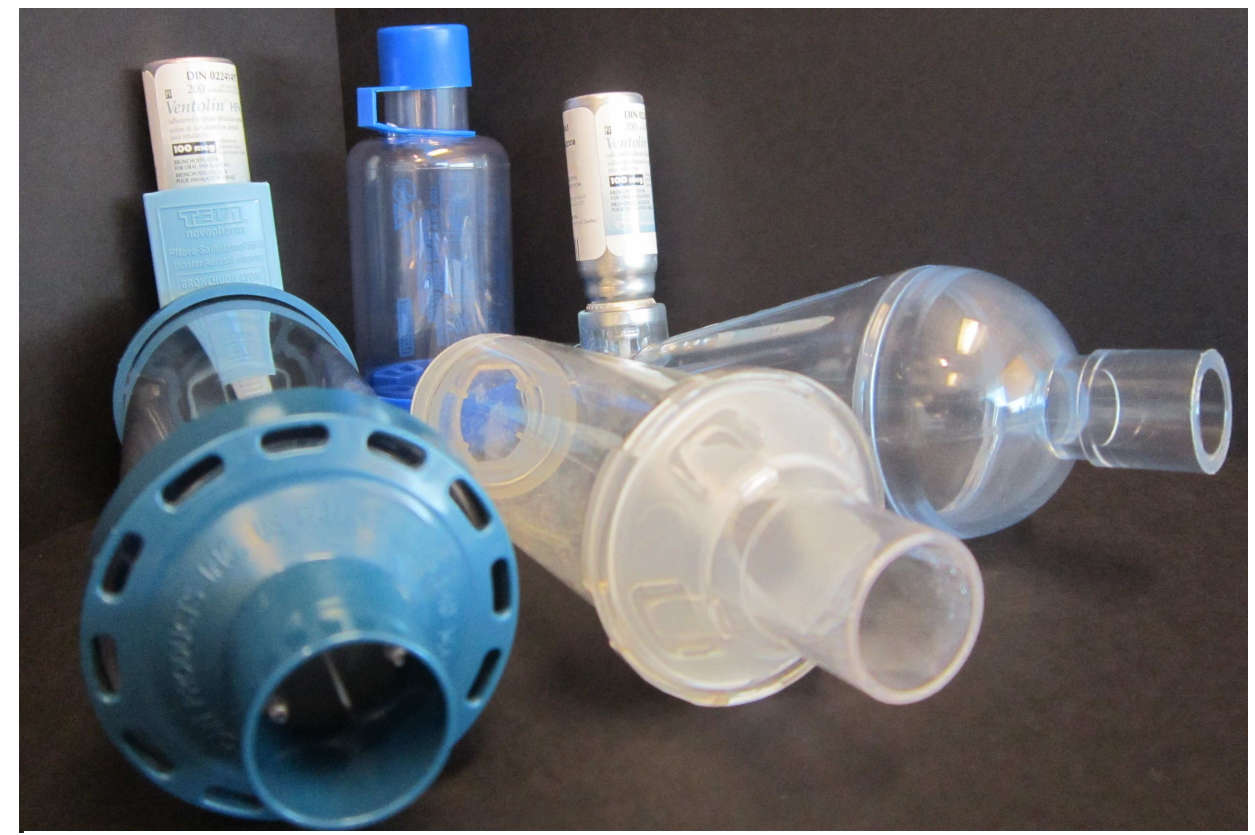

Figure 1.3: Photographs of Add-On devices (Spacers).

Most spacers reduce oral deposition and provide limited protection against poor hand-breath coordination. Particle characteristic, such as the size of particles reaching the patient, are affected by the spacer shape and size [22]. In such devices aerosol mechanics are governed by the equations of motion, droplet evaporation and the NavierStokes equations governing the fluid motion of a turbulent gas jet composed of the propellant and air actuated in those types of devices [30].

VHCs are spacers that comprise a one-way, low-resistance valve that allows the aerosol spray to remain within the device and lets the particles slowly settle until the patient inhalation effort opens the valve, VHCs are partially breath-actuated. Advantages of VHCs include decreasing the overall size of the spray particles (as the larger particles collide with chamber walls), revoking the cold-Freon effect, and leading to an improved coordination with aspiratory flow [31]. Some delay is allowed by the design 
of the holding chamber and therefore, the dose that is emitted from the pMDI into the chamber should be inhaled very soon after it has been discharged; for each inhalation only one single actuation should be released into the chamber [32] and [33].

Modification may be required for VHC designs for the new HFA pMDIs. For instance, it becomes clear that some HFA formulations generate a slower particle velocity, smaller particle size, and a higher aerosol temperature. The quality of the aerosol spray could possibly be influenced by these changes [34].

Most of children under 4 years of age lack the coordination necessary to use their inhaler devices [35]. Generally, it is recommended that young children use their inhalers with a spacer device and face mask. Figure 1.4 shows the ODAPT soft mist adapter and the EcoMask facemask. The ODAPT soft mist adapter (McArthur Medical Sales Inc., Rockton, ON) was designed to deliver inhaled medication via pMDIs and Respimat SMIs to patients requiring a facemask or tracheostomy application.

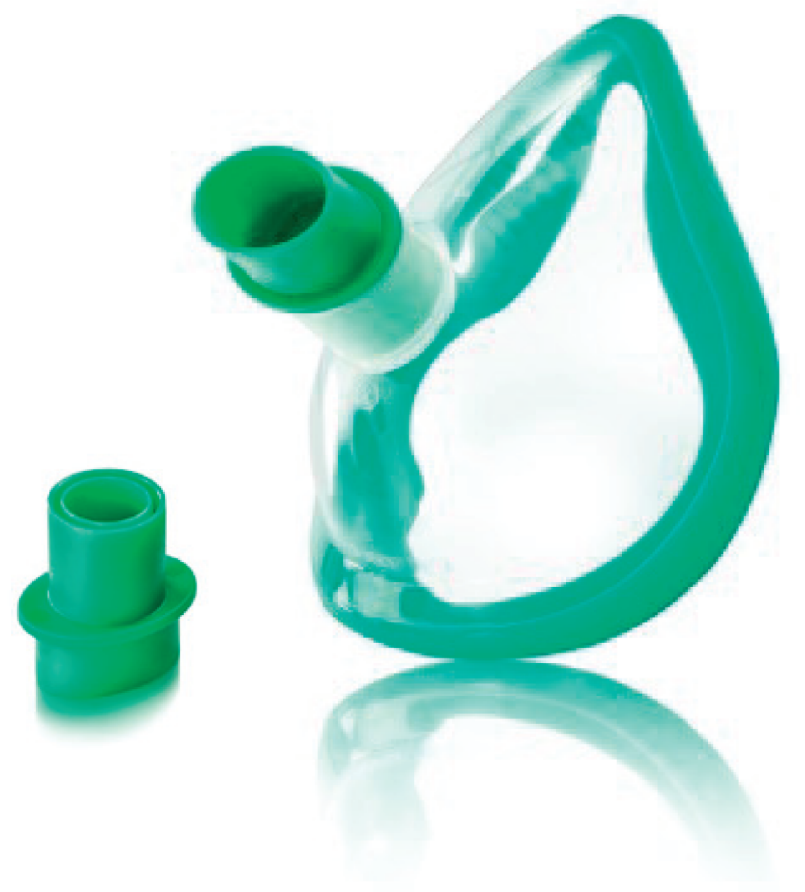

Figure 1.4: Add-on devices: ODAPT soft mist adapter (left) and EcoMask facemask with ODAPT adapter (right). 


\subsection{Soft Mist Inhalers (SMIs)}

Soft Mist Inhalers (SMIs) are a new-generation of propellant free inhalers. This type of inhalation device is classified under nebulizer as it is used to produce droplets, which are suitable for inhalation, by transformation of an aqueous liquid solution to liquid aerosol particles [36]. The first delivery inhalation spray system of this kind was the Spiriva Respimat inhaler (Figure 1.5 Boehringer Ingelheims, Germany) in 2004 [36-39]. Spiriva Respimat inhaler has its advantages when compared to pMDIs such as portability, compactness, high lung deposition and instead of comprising of a propellant it contains a compressed spring inside the housing, which powers the device [37,38]. To actuate the device the patient has to twist the base of the inhaler. This process will meter out a dose and compress the spring, as mentioned earlier, to provide the energy source to deliver the medication. To deliver the dose the patient must press the doserelease button. This will force the medication through a complex nozzle configuration to generate a respirable aerosol [38]. Each actuation delivers $2.5 \mu \mathrm{g}$ of tiotropium bromide monohydrate which is the active medication of the Spiriva Respimat inhaler.
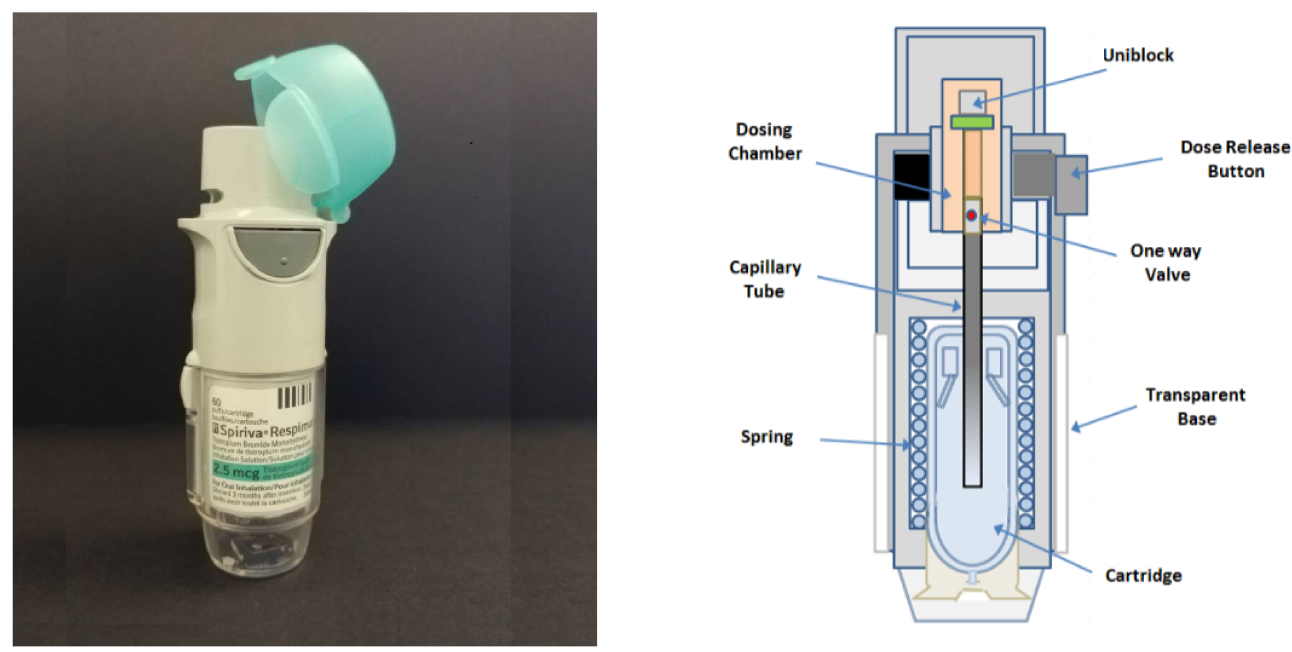

Figure 1.5: The Respimat softmist inhaler. 


\subsection{Characterization of pMDI Spray Velocities}

\subsubsection{Experimental study}

There is not an abundance of in-depth research on the spray generation mechanism of the pMDI, regardless of its beneficial implication and therapeutic consequences [10, 30]. Also, the real spray from a pMDI must be correctly characterized prior to any experimental or numerical simulations. Some internal and external spray characteristics in the far-field $(x / D \geq 50)$ have been documented in a number of studies.

The first report of a flow visualization study was performed by Versteeg and Hargrave [9], regarding the transient flow and primary atomisation procedure of a pharmaceutical MDI; highlighting its complexities. Also, Versteeg and co-workers [10], used visualization techniques and reported that there was a circular flow regime along with a vapour core and an unsteady wall film composed of a foamy liquid inside the expansion chamber of the rectangular model, which was in agreement with their first study. Their visualization study demonstrated that the flow in the expansion chamber is heterogeneous with some flash boiling and bubble formation. Furthermore, production of the primary spray formation mainly takes place close to the spray orifice exit.

Wigley et al. [11], performed Phase-Doppler Anemometry (PDA) on an HFA-227propelled placebo and active formulations. The experimental plane of measurement was taken at an $x / D$ of 6.2 relative to the nozzle orifice, to find the axial and radial velocity components, the arithmetic and Sauter mean diameter profiles, which is the ratio of volume to surface area of a spherical droplet to model the particles of interest in a complete spray [40]. The spray characteristics in the near-orifice region illustrate that a highly pre-atomised spray with a typical mean droplet size $D_{10}$ between 2 and $5 \mu m$ arises from the actuator orifice. Also, it was shown that the spray exhibited a complex temporal behavior which included a significant transient movement normal to the main spray direction. 
Most of the comprehensive research on pMDI devices to measure the velocity of the particles at discrete points in the flux field have been performed by Dunbar and co-workers $[12,13,16]$. A theoretical and experimental analysis of pMDI spray characteristics has been made possible by utilizing Phase Doppler Particle analysis. As a result of this measuring technique, droplet velocity, size (assuming spherical droplets) and concentration data have been successfully presented. This work was done during the search for suitable and convenient propellant that would replace chlorofluorocarbonbased propellants being used at the time. For this reason, the work was completed using custom-manufactured canisters containing only an HFA-placebo formulation.

An important aspect of this work is the comparison of the measured spray velocity with numerically-simulated spray velocities. The measured and simulated velocity profile at the center of the mouthpiece, in the mouthpiece plane, is reproduced in Figure 1.6 .

In carrying out this research, a droplet model, where the gas phase and the dispersed liquid phase are illustrated individually. Also, to model turbulent dispersion of the droplets the eddy interaction type of Gosman and Ioannides [41] was employed. The high-Reynolds number $k-\varepsilon$ turbulence model of Launder and Spalding [42] was also utilized for the continuous gas phase. The computational domain that was used was a two-dimensional axisymmetric structured grid composed of 70 nodes in the stream-wise direction and 25 nodes in the vertical direction. The findings of the experimental results illustrated two local velocity maxima in time, whereas the numerical results provided only one. It was assumed that the extreme spray density in the near-orifice area of the spray was the main origin of the error. In contrast, Phase Doppler Anemometry measurements of a placebo pMDI with just HFA-227 propellant performed by Wigley [11], clearly showed that a second velocity maxima in time is the same as the experimental results of Dunbar and co-workers.

Versteeg et al. [15] used three types of techniques to characterize the spray plume 
issued from Presspart $19 \mathrm{ml}$ canisters (Presspart, Blackburn, UK), in areas near the spray orifice and away downstream. The techniques used were PDA, high-speed imaging and particle image velocimetry (PIV). The high-speed imaging captured the overall characteristics of the spray plume and the cone angle was measured at four-time intervals $50,100,150$, and $200 \mathrm{~ms}$. It showed that the angle was $60^{\circ}$ at $50 \mathrm{~ms}$ with an extremely dense spray. Spray density in the core reduced with time until the core angle reached $20^{\circ}$ at time $150-200 \mathrm{~ms}$ in the near orifice region. At the beginning of the far-field region, the cone angle was around $20^{\circ}$ and declined to $10^{\circ}$ through the final stages of the spray event.

At $25 \mathrm{~mm}$ from the spray orifice the PDA temporal velocity profile at the spray centerline displayed a high initial velocity of $36 \mathrm{~m} / \mathrm{s}$, then it decreased sharply to a minimum near a time of $20-40 \mathrm{~ms}$. The velocity slowly raised to a maximum peak around $100 \mathrm{~ms}$ and afterward declined gradually to the end of the spray event as presented in Figure 1.6. In addition, the velocities measured by both of PDA and PIV systems were in close agreement during the spray event except after $200 \mathrm{~ms}$, where PIV did not give any velocity information. The droplet size $D_{10}$ measured by the PDA at the same location was found to be around $2-4 \mu \mathrm{m}$.

Kakade et al. [43], investigated their novel pMDI actuator system; the vortex nozzle actuator (VNA). Measurements were performed with Laser Doppler Anemometry configuration along with a Phase Doppler Anemometry system (PDA), which is an optical diagnostics, laser-based, high-speed video imaging system used to measure actuationaveraged velocities of the pMDI spray. In that study a comparison was made between VNA measurements against the conventional pMDI actuator which is the Proventilbrand salbutamol pMDI. The results show that velocities of the novel design were much lower than conventional pMDI actuators. Moreover, for the conventional pMDI actuator in the domain between 3 and $7 \mathrm{~mm}$ below the horizontal plane, which stretched about $2.5 \mathrm{~mm}$ left and right of the central vertical (sagittal) plane, there was a peak 


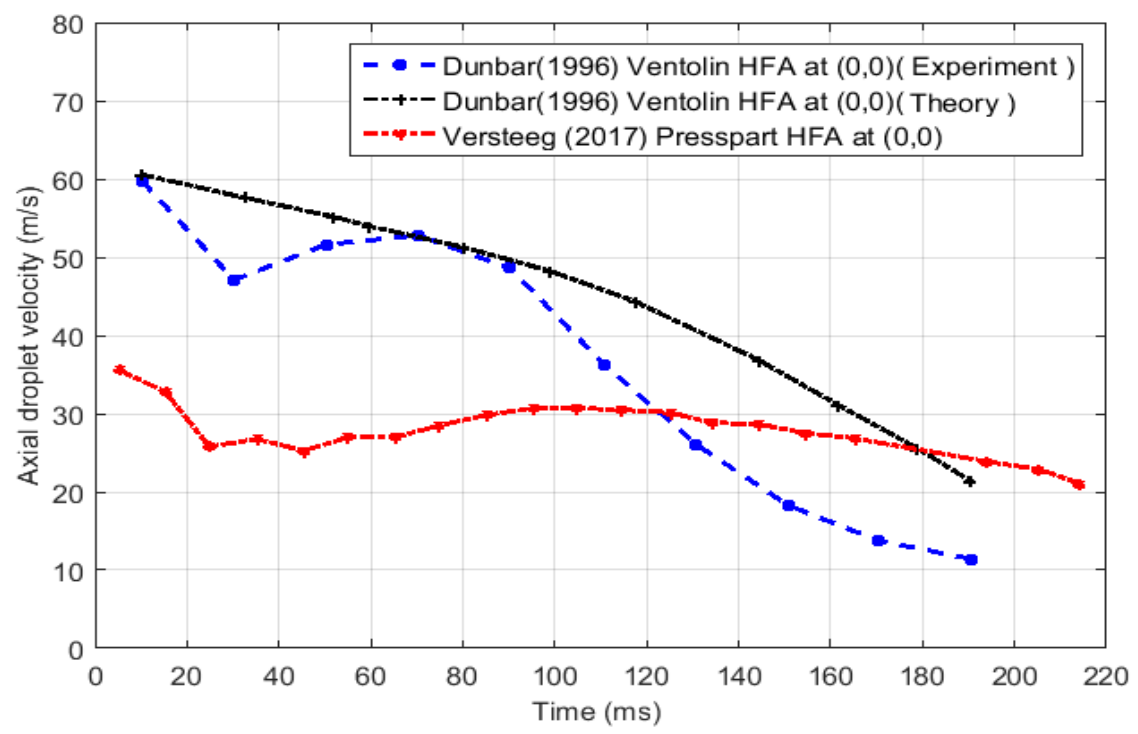

Figure 1.6: Droplet velocity in the center of the mouthpiece exit plane. Adapted from $([12],[15])$.

actuation averaged velocity of $13.5 \mathrm{~m} / \mathrm{s}$.

Crosland et al. [14] used a PIV system to measure the spray particle velocity in a 20 vs. $16 \mathrm{~mm}$ window at the exit of the salbutamol sulfate pMDI mouthpiece, after a pneumatically controlled actuation. Through the spray event measurements were made and were ensemble-averaged at nine varied times ranging from 1.5 to $100 \mathrm{~ms}$. It was found that the spray was extremely unsteady, and had strong velocity gradients in the radial direction. In the first $100 \mathrm{~ms}$ of the transient spray event, the direction and cone angle of the spray were unsteady as well, varying between $6^{\circ}$ and $8^{\circ}$ respectively. This study and its results are comparable to the results that were obtained by Wigley [11], as well as experimentally (but not numerically) by Dunbar [12] as can be seen in Figure 1.7. The results are also in agreement with the velocity maps which were demonstrated by Kakade [43] in the placement of the peak velocity below the horizontal (xz) plane.

However, Crosland used a PIV system to visualize this event. The velocity vector flow-field and selected radial profiles of axial velocity were plotted for three time delays for actuation into quiescent air; this can be seen very clearly in Figure 1.8, where it 
shows the deflection in the pMDI spray.

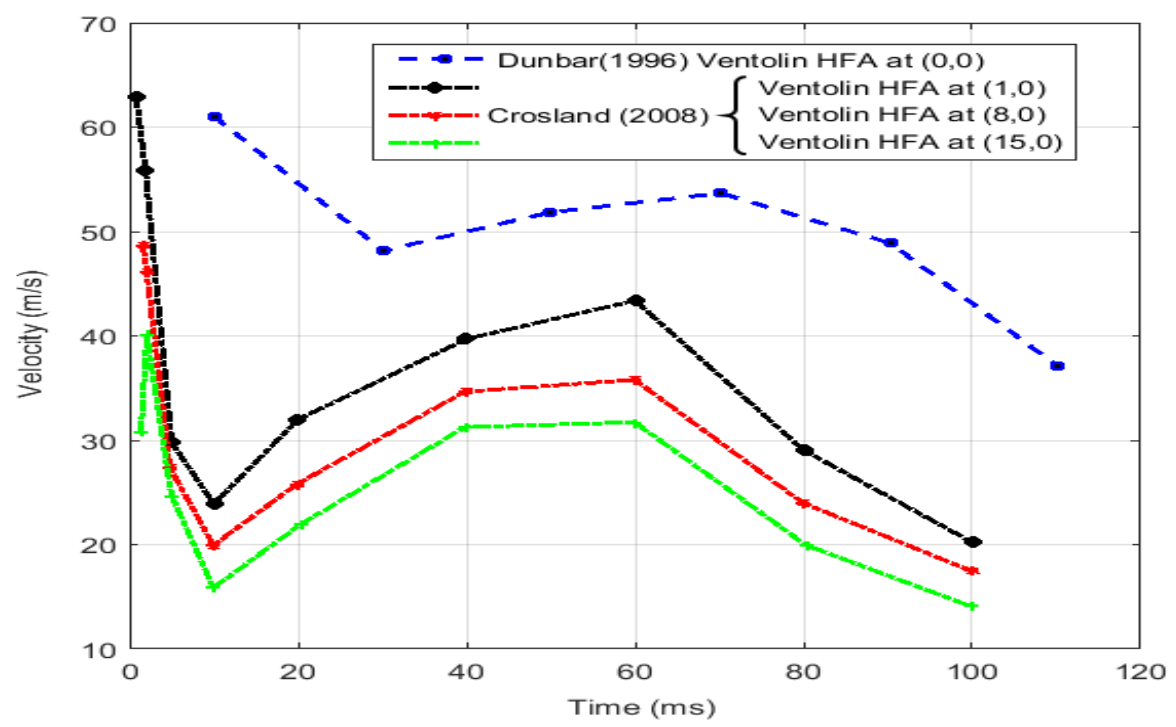

Figure 1.7: Temporal evolution of the spray velocity as emitted from a Ventolin-brand pMDI. Adapted from [7].

Smyth et al. [44] carried out an experimental research to classify the device factors that have an impact on both particle size profiles and spray pattern as shown in Figure 1.9. The selected actuator features were the orifice diameter, the expansion chamber depth and orifice length. The orifice diameters were 0.25 and $0.31 \mathrm{~mm}$, expansion chamber depth diameters were 7.55 and $8.15 \mathrm{~mm}$, and orifice lengths were 0.7 and 0.9 $m m$. Custom-built actuators were used to limit the influence of actuation on the results. Their analyses were conducted through a laser light sheet illumination method for spray patterns and laser diffraction for particle size analysis. In their findings, not only spray patterns were considerably affected by the actuator orifice length, sump depth, and orifice diameter, but also particle size was significantly influenced. In addition, they concluded that those factors did not contribute independently to particle size distributions and that there were significant interactions between them.

Liu et al. [17] carried out a research by using PDA to examine the instantaneous velocity of the discharged droplets from pMDIs in addition to the corresponding droplet 
size distribution by using a Laser Light Scattering (LLS) system. That study compares those characteristics for pMDIs of both formulations of CFC and HFA. The measurements were made at two discrete points $30 \mathrm{~mm}$ and $60 \mathrm{~mm}$ from the front of the pMDI mouthpiece. The mean droplet velocities that were found at those two testing distances were in the range of 5.4 to $20.1 \mathrm{~m} / \mathrm{s}$ and 4.7 to $11.7 \mathrm{~m} / \mathrm{s}$, respectively.

For all tested pMDIs, it was found that when the distance increased there was a decline in the droplet velocity; with CFC formulations showing a larger decrease than HFA formulations. Mixed results were found when comparing HFA and CFC MDI products, as the droplet size appears to be influenced more by the valve and orifice design than by the propellant. Droplet sizes $\left(D_{10}, D_{30}\right.$, and $\left.D_{50}\right)$ for all tested pMDIs were statistically different from each other. The droplet size parameters measured by both PDA and LLS were smaller at $60 \mathrm{~mm}$ than those measured at $30 \mathrm{~mm}$. Furthermore, their findings were in good agreement with those of Dunbar $[12,13,16]$.

Pitcairn et al. [45] carried out a study between different medications including Respimat and pMDI. The Respimat contained a budesonide solution and the pMDI comprised a beclomethasone dipropionate. The objectives of that study was to find the deposition pattern within the lungs. The mean percentage dosage of steroid deposited was $52 \%$ for Respimat and $9 \%$ for the pMDI. In addition, it was concluded that lower doses of drugs delivered by the Respimat would provide equal efficacy to higher doses delivered through the pMDI [46]. That result is due to the large percentage of the droplets emitted by Respimat that fall into the fine particle fraction classification (droplets with a diameter less than $5.8 \mu m$ ) $[47,48]$.

Hochrainer et al. [49] conducted an investigation to find the spray velocity and spray duration, using Respimat ${ }^{\circledR}$ SMI and a variety of chlorofluorocarbon $(\mathrm{CFC})$ and hydrofluoroalkane (HFA) pMDIs. The aerosol mean velocity was measured by a video recording technique which was taken at $100 \mathrm{~mm}$ away from the nozzle. The determination of the spray duration was performed via three approaches: video recording, laser 


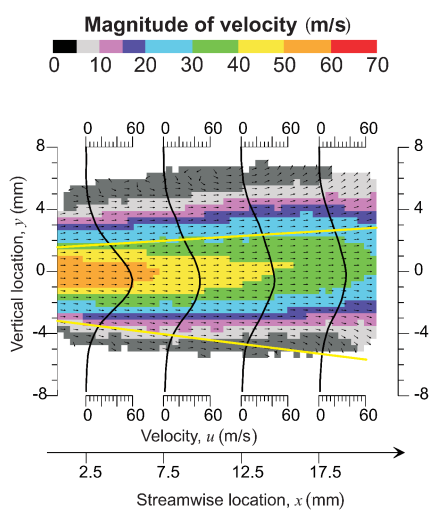

(a) At $2 \mathrm{~ms}$ after actuation.

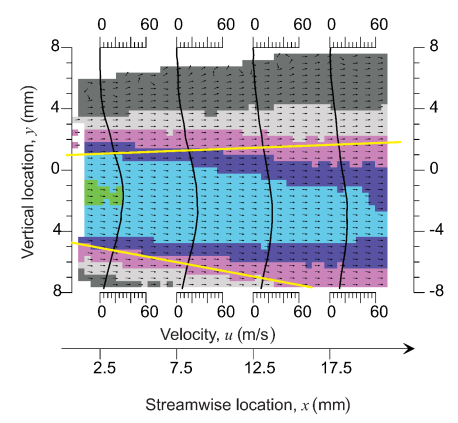

(b) At $10 \mathrm{~ms}$ after actuation..

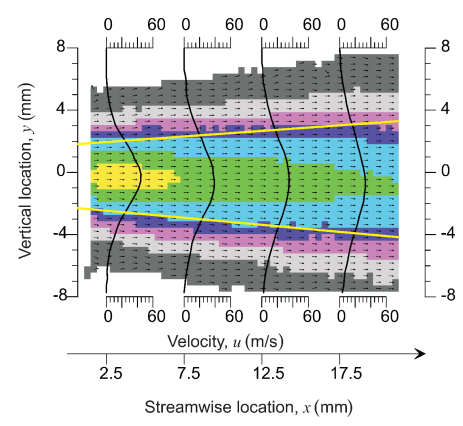

(c) At $40 \mathrm{~ms}$ after actuation.

Figure 1.8: The velocity vector flow-field and selected radial (vertical) profiles of axial velocity for three-time delay actuations into the quiescent air. adapted from [14]. 


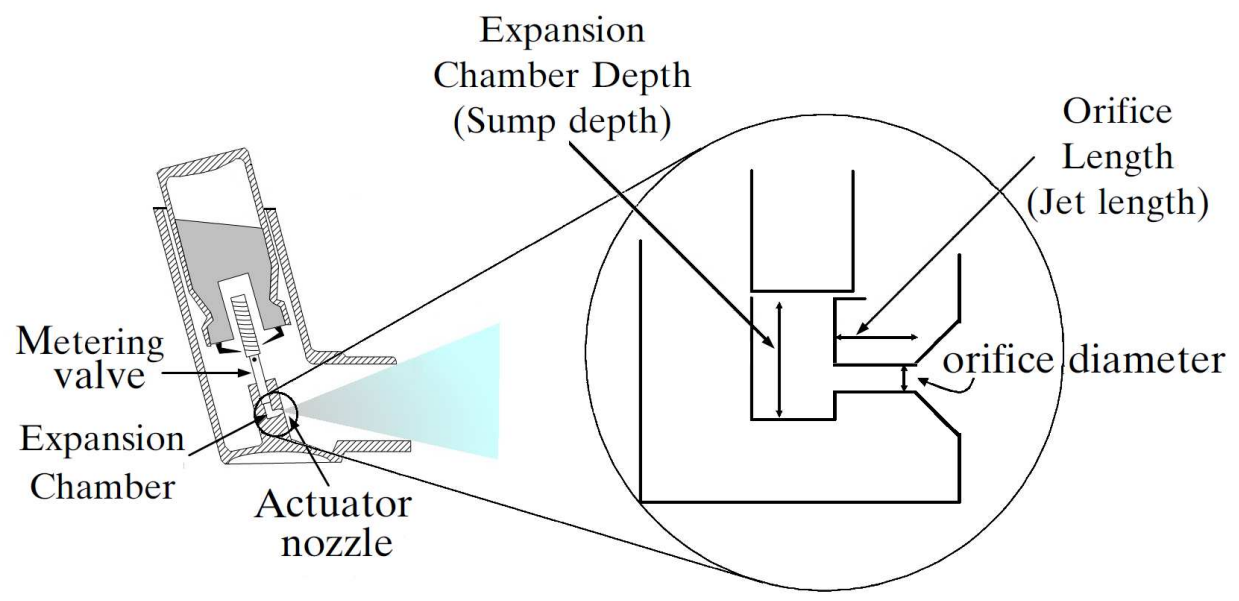

Figure 1.9: Schematic of the three factors of the pMDI actuator orifice. adapted from [44].

light diffraction and rotating disc. However, the evaluation of the spray duration of Respimat SMI with other pMDI inhalers was achieved by the Video recording method. Their findings for the mean velocity of the Respimat SMI was $0.8 \mathrm{~m} / \mathrm{s}$, whereas, it was in the range between 2.0 to $8.4 \mathrm{~m} / \mathrm{s}$ for the pMDIs. The mean duration for the spray cloud was $1.5 s$ for the Respimat SMI, and 0.15-0.36 $s$ for the pMDI inhalers.

\subsubsection{Numerical Studies}

The use of computational fluid dynamics (CFD) to model air flow in addition to the transport and deposition of aerosols in the extrathoracic airway, plays a very important part in prediction of the behavior and the response of the system which is being investigated. Recently, many attempts have been performed by a number of researchers to use CFD analysis to explore the effects of some factors and conditions affecting pMDIs. Most research has been executed through the particle transport problem; partially decoupled in such a way that in steady-flow the simulation of the air-flow and subsequently tracking the simulated release of particles contained by the resolved fluid 
domain. This procedure was performed by Heenan et al. [50], Farkas et al. [51], and Jin et al. [52]. Also, in the study of unsteady breathing flow, by employing breathing waveforms to represent three different inhalation patterns, Takano et al. [53] used this method to simulate the flow-field and particle deposition. In their study, the slip boundary condition was set to zero for the released particles. On one side, in such a process, the release of particles with more appropriate initial conditions would benefit the modeling of pMDI sprays. On the other hand, there would be some limitations due to the insufficiency of elaborated data to efficiently model the time-dependent velocity boundary conditions of the pMDI spray.

In their study, Heenan et al. [54] carried out a research on the air flow in an idealized human oropharynx in both, experimental and numerical simulations. The study was carried out for three inlet velocity profiles with small different inlet velocities, and each at volume flow rates of 15,30 and $90 \mathrm{l} / \mathrm{min}$. These profiles were utilized to find their effect on the resulting flow field. Their finding was that the velocity profiles near the oropharynx varied from $3 \%$ to $6 \%$ of the ideal plug flow profile at that point, which could be presumed as a result of the effects of a transient boundary condition, would be much more significant.

Most comprehensive work on the numerical simulation of the pMDI flow was conducted by Kleinstreuer et al. [55]. Most previous simulation studies were performed by releasing droplets into a previously calculated flow-field, however, this study was on simulated, droplet breakup and evaporation. They used Reynolds-Averaged Navier-Stokes with a low-Reynolds number $k-\omega$ turbulence model as parameters in the simulation. A generic spacer, followed by a simulated human upper airway for the flow was created as a numeric model. The simulation carried out via HFA-propellant properties using a nozzle diameter of $0.25 \mathrm{~mm}$, and with CFC-propellant properties using two nozzle diameters of $0.25 \mathrm{~mm}$ and $0.50 \mathrm{~mm}$. Results showed that simulated lung deposition accomplished using the HFA-propelled pMDI with the smaller orifice diameter by $46.6 \%$, 
whereas, it was $23.2 \%$ for CFC-propelled pMDI with the same nozzle diameter. Increasing the diameter for CFC-propelled pMDI reduced the simulated lung deposition to $5.2 \%$ only.

Longest et al. [56] used in-vitro experiments and a previously developed CFD model to determine the effects of spray aerosol generation time on transport and deposition in a standard induction port (IP) and a more realistic mouth-throat (MT) geometry. The investigation studied capillary aerosol generation (CAG) which acted as a representative system in which spray momentum was expected to considerably impact deposition. To examine the depositions (in both the sectional and the total) in the IP and MT geometries, a flow rate of $25 \mathrm{mg} / \mathrm{s}$ for aerosol generation times of 1,2 , and $4 \mathrm{~s}$ were selected. Their finding was a noticeable reduction in the deposition fraction in the IP and MT geometries by approximately 60 and $33 \%$, respectively, when the generation time of the CAG system was raised from 1 to $4 \mathrm{~s}$. Moreover, the CFD predictions of deposition fraction were found to be in good agreement with the in-vitro results for all times considered in both the IP and MT geometries.

Al-Hegagi [57], studied the deposition of salbutamol sulphate aerosol spray from Ventolin ${ }^{\circledR}$ pMDI experimentally and numerically in three types of add-on spacer devices, which were OptiHaler, OptiChamber and PocketChamber, for flow rates of 30, 45 and $60 \mathrm{~L} / \mathrm{min}$. Salbutamol sulphate deposition was analyzed by a spectrophotometer. The experimental results demonstrated that the smaller OptiHaler and PocketChamber acquired a greater amount of drug deposition in the spacer, whereas the comparatively large volume OptiChamber presented less drug deposition in the spacer and more drug available for inhalation. It also showed that the drug deposition in the lower half of the OptiChamber was higher than the upper half. Furthermore, RANS equations and Eddy Interaction models (for the particulate phase) together with the Shear Stress Transport (SST) turbulence model were used in the numerical simulation to define the effect of the spray angle on the deposition of Salbutamol Sulphate in the OptiChamber spacer. 
The results showed higher drug deposition in the lower half of the OptiChamber than the upper half (using a $6^{\circ}$ downward spray angle and $30 \mathrm{~L} / \mathrm{min}$ flow rate).

Oliveira et al. [58] carried out a numerical simulation on Ventolin ${ }^{\circledR}$ propellant HFA 134a, with particles diameters ranging from $1.22 \mu m$ to $49.5 \mu m$ using an $8^{\circ}$ spray angle in a Rosin-Rammler distribution. The parameters were tested in Fluent ${ }^{T M}$ (v6.3.26), for a flow rate of $1 e^{-6} \mathrm{~kg} / \mathrm{s}$. Their remarks were that the selected parameters produced a simulated spray very similar and in agreement to the experimental values found in previous experimental studies. In addition, the air behavior had a predictable response. The results showed that the particles reduce their injection velocity from $40 \mathrm{~m} / \mathrm{s}$ to the air velocity of $0.05 \mathrm{~m} / \mathrm{s}$ in a very short time. Moreover, air velocity increased, as well as a perturbation of the air velocity field. This effect is due to the momentum transferred from the fast moving droplets into the main flow. This effect is only observed in the vicinity of the spray.

In a recent study Ogrodnik et al. [59], determined medication (salbutamol sulfate) deposition within both a large and a small-volume spacer (the large-volume spacer used was the $750 \mathrm{ml}$ volume Volumatic spacer, and a $218 \mathrm{ml}$ volume OptiChamber for the small-volume spacer), through an experimental and numerical analysis. The experiments were conducted with the same technique used by Al-Hegagi [57] through an application of spectrophotometry. Computational fluid dynamics was used to calculate the numerical deposition. This was carried out by implementing mean flow and turbulent tracking of particles using unsteady Reynolds-Averaged Navier-Stokes (URANS) equations with a shear stress transport turbulence model. Their finding was that the deposition of the medication was to be greater in the lower half as opposed to the upper half of both spacers experimentally and numerically because of a downward spray angle. In addition, the numerical simulation results showed that inertial impaction is the most likely method of deposition for the Volumatic spacer, and turbulence is more likely to cause deposition in the OptiChamber spacer. 
Longest et al. [38], carried both computational fluid dynamics (CFD) model and in vitro experiments, to characterize the transport and deposition of an aerosol released from the Respimat SMI inhaler. The Deposition of the Respimat medication was studied in the inhaler mouthpiece (MP), a standard induction port (IP), and mouth-throat (MT) geometry at a fixed inhalation flow rate of $30 \mathrm{l} / \mathrm{min}$. Laser diffraction method was used in the experiments to measure the initial polydisperse particle size distribution. The results showed that the numerical predictions of deposition fractions were in a good agreement with in vitro results, for both IP and MT geometries. MP has most of the medication loss (deposition) and ranged from 27 to $29 \%$ as measured by the experimental work. This loss in the MP was as a result of recirculating flow pattern that surrounded the aerosol spray and transported a large number of small droplets, as reveled in the CFD simulation. In addition, results from CFD simulation showed that both IP and MT geometries had deposition of $4.2 \%$ and $7.4 \%$ respectively, which is comparatively low to the experimental data. 


\section{Chapter 2}

\section{Methods of Aerosol Particle Size Characterisation}

\section{$2.1 \quad$ Introduction}

Droplet or particle size is the most significant factor that impacts the deposition of aerosols in the lungs [49]. Therefore, to have a good understanding and meaningful interpretation of the particle size data, it is important to be aware of the principles, theory and the methods of estimating particle sizes [60]. To measure the particle size of aerosols there are a wide variety of techniques that have been used. Generally, these methods result in the measure of a particular diameter that may be defined according to the principle that underlines the measurement [61].

The aerodynamic diameter of a particle is a diameter of a unit-density $\left(1 \mathrm{~g} / \mathrm{cm}^{3}\right)$, sphere comprising of the same settling velocity as the particle, normally measured in air. This parameter includes factors such as the particle shape, density, and physical size, which reflect on the aerodynamic behavior of the particle. While a dynamic parameter, it can generally be linked with aerosol deposition; specifically with the deposition occurring in the lung [62]. 
Particles that are the same size are seldom found in aerosols, therefore the mean size is typically used to describe an observation of a distribution of sizes. This observed data can be fitted by the statistical approximation of a distribution. Frequently, when plotting a number of particles in a size range against the logarithm of the particle diameters, the distribution exhibits a normal (Gaussian) trend.

The following sections focus on the principles of characterisation of pharmaceutical aerosols by use of Cascade Impactor or Laser and Phase Doppler Anemometry.

\subsection{Particle Characterization}

There are a variety of apparatuses available on the market to measure fine particles. However, the cascade impactor is the preferred option used to characterize active pharmaceutical ingredient by the pharmaceutical industry and described in the United States Pharmacopeia, European Pharmacopeia, and British Pharmacopeia [63]. Its principle is based on a mixture of gas and the particles which is then directed through the impactor nozzle. As illustrated in Figure 2.1 (for one stage), the jet streamlines curve sharply as a result of the plate that is placed close to the nozzle. Depending on the particles' inertia, the larger particles will depart from the streamlines, and collected due to their impingement on the plate; others with small inertia are driven away by the gas stream to the next stage, as displayed in Figure 2.2. To increase the jet velocity, the nozzle diameter is made gradually smaller from one stage to the next. Therefore, particles with different sizes can be collected and separated on each stage consecutively [64].

Particle characterization using cascade impactors are time-consuming (cleaning, drying, assembling, testing, and analyzing of medication deposition on each plate). PDA (Phase Doppler Anemometry) represents an alternative to cascade impactors, despite its high cost [65].

As mentioned earlier, the particle size of a pharmaceutical aerosol is a significant 


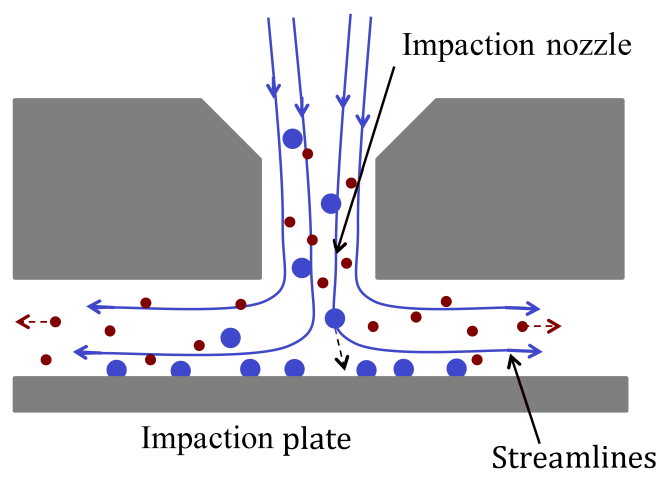

Figure 2.1: Schematic diagram of Cascade Impactor.Adapted from [64]

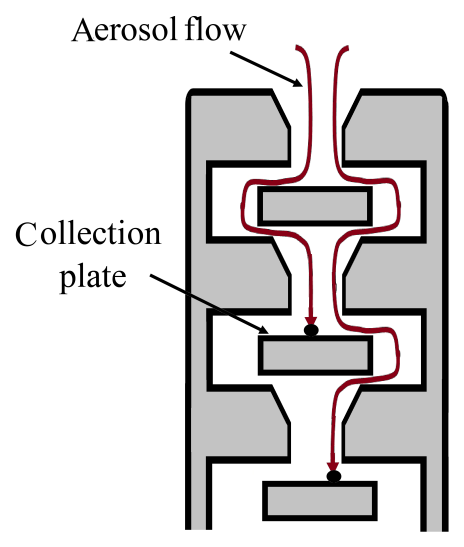

Figure 2.2: Inertial impaction of particles and airflow in ACI. 
parameter, since the patient can only benefit from fine particles that are delivered to the lungs [66]. Particle size is defined by several parameters including the arithmetic mean diameter $\left(D_{10}\right)$, the volumetric mean diameter $\left(D_{30}\right)$, and volume-based median diameter $\left(D_{\mathrm{v} 50}\right)$.

The arithmetic mean diameter, $D_{10}$, is the simple average diameter of all the collected particles in a spray, and is gained through the following equation [67]:

$$
D_{10}=\frac{\sum_{i=1}^{p} n_{i} D_{i}}{\sum_{i=1}^{p} n_{i}}
$$

Where, $n_{i}$ means the (number-based) frequency of occurrence of particles in size class $i$, having a mean diameter $D_{i}$, and $p$ the total number of particles.

The volume mean diameter $D_{30}$ is calculated from the mean of the particle volumes and is determined by [67]:

$$
D_{30}=\sqrt[3]{\frac{\sum_{i=1}^{p} n_{i} D_{i}^{3}}{\sum_{i=1}^{p} n_{i}}}
$$

Within an aerosol, fifty percent of its particles, and of both its volume, and mass, is contained equally above and below what are termed the volume median diameter $D_{\mathrm{v} 50}(\mathrm{VMD})$ and mass median diameter (MMD). Where particle density is consistent throughout the aerosol, VMD and MMD are the same [68] [69].

Also, the moving average is a method used to get an overall understanding of the trends in a data set which can be employed for both averaging over time or averaging over data. That for a given sequence $\{a\}_{i=1}^{N}$, an $n$-moving average is a new sequence $\left\{s_{i}\right\}_{i=1}^{N-n+1}$ defined from the $\left\{a_{i}\right\}$ by taking the arithmetic mean of subsequences of $n-$ terms $[70,71]$,

$$
s_{i}=\frac{1}{n} \sum_{j=i}^{i+n-1} a_{j}
$$




\subsection{Andersen Cascade Impactor (ACI)}

The ACI consists of a system of 8 functional stages that will filter the particles based on their aerodynamic size. These stages are designated as stage 0 to stage 7 , sorted from the largest to smallest particle size bin. Each stage consists of two parts, the stage itself and a collection plate that is placed under the stage, as displayed in Figure 2.3. The sieve of stage 0 consists of the largest holes of the apparatus and each successive sieve consists of increasingly smaller holes with the smallest ones located on stage 7 . When the ACI is assembled, the stages are placed one on top of another, starting with the filter for the largest particles at the top and the smallest at the bottom. It also includes a level F, which acts to properly seal level 6, and a $90^{\circ}$ elbow (induction port) that is connected to level 0 . The whole system is then connected to a vacuum pump that draws air at a required flow rate. Figure 2.4 displays the assembled ACI.

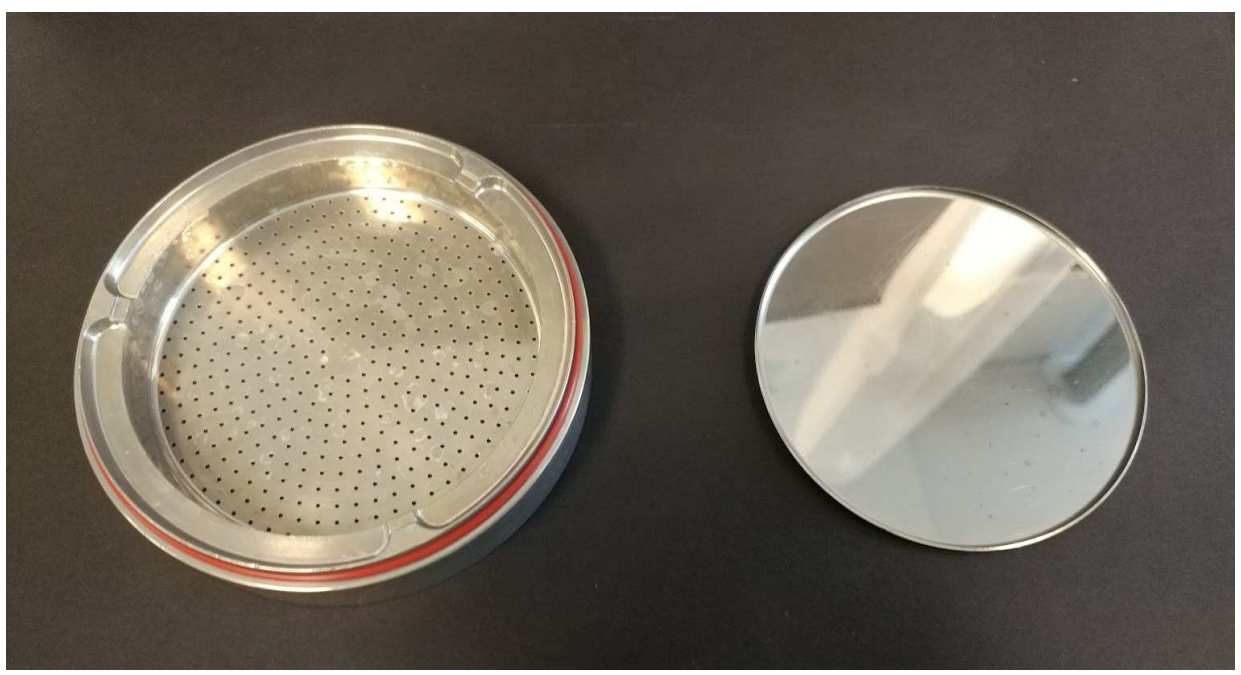

Figure 2.3: ACI stage, consisting of sieve (left) and the respective collection plate (right). 


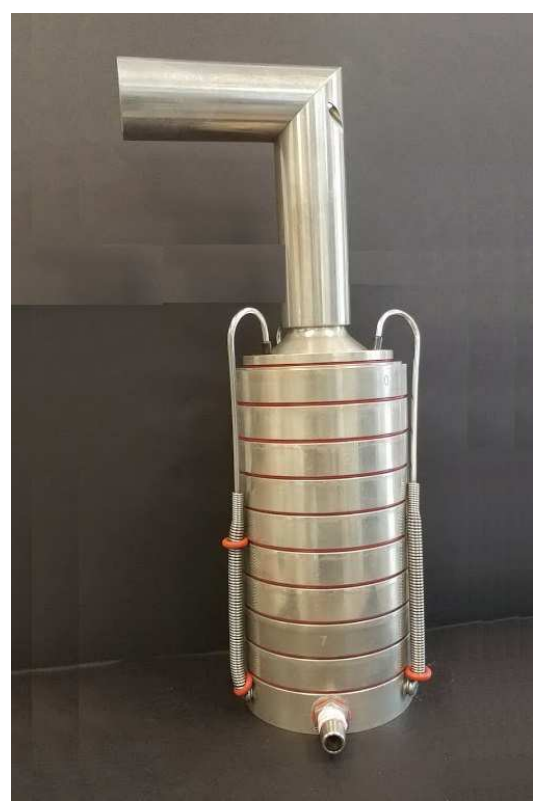

Figure 2.4: Assembled Andersen Cascade Impactor.

\subsection{Theory of Laser and Phase Doppler Anemometry (PDA)}

The measurement technique of Phase Doppler Anemometry has been used for decades. This technique was introduced in the mid-1960s, although it was only in the 1970s that it came into prevalent use and since became a well established optical technique for the measurements of particle velocities [72]. In 1975, Durst and Zaré were the first researchers to performed a successful PDA fluid measurement experiment, in which they found the velocity and size distribution of suspended fluid particles simultaneously in an effective way [73].

PDA uses a point measurement, which is also a non-intrusive fluid measurement technique used to characterize the size and the velocity of individual spherical particles. This system is widely used as a technique for fluid dynamic investigations in gases and liquids or other types of jets, in order to characterize the aerosols. The development of the PDA was based on the Laser Doppler Anemometry (LDA) and therefore the PDA 
can be considered as an extension of this. The essential principle of the Laser Doppler Anemometry (LDA) is to measure the velocity of seeded tracer particles carried by the flow. If the size of these particles is within the range of measurements, their velocity is presumed to be that of the stream and the LDA provides the measurements of the local instantaneous velocity, the mean velocity and the turbulent quantities for these particles.

\subsubsection{The Measurement Volume}

The measurement volume is the region of fringes that is formed by the intersection of two coherent monochromatic light beams, as illustrated in Figure 2.5. The particle velocity and size statistic is contained in the scattered field of light when particles cross the measurement volume due to the Doppler Effect [74].

Moreover, an important parameter of the LDA system is the size of the measurement volume, as it defines the spatial resolution of this technique. Figure 2.6 shows that the measurement volume is an ellipsoid due to the Gaussian intensity distribution of the beams in all three dimensions. The dimensions of the measurement volume are defined by the light intensity distribution of the fringe pattern [72].

\subsection{Laser Doppler Anemometry}

The LDA system is considered non-intrusive and direction sensitive. Its principles make it very appropriate for application in a number of different fields, for instance medical applications and spray diagnostics. The advantages of this technique are mainly the non-intrusive measurement, high spatial and temporal resolution, there is no necessity for calibration and it has the ability to measure reversing flows [75]. 


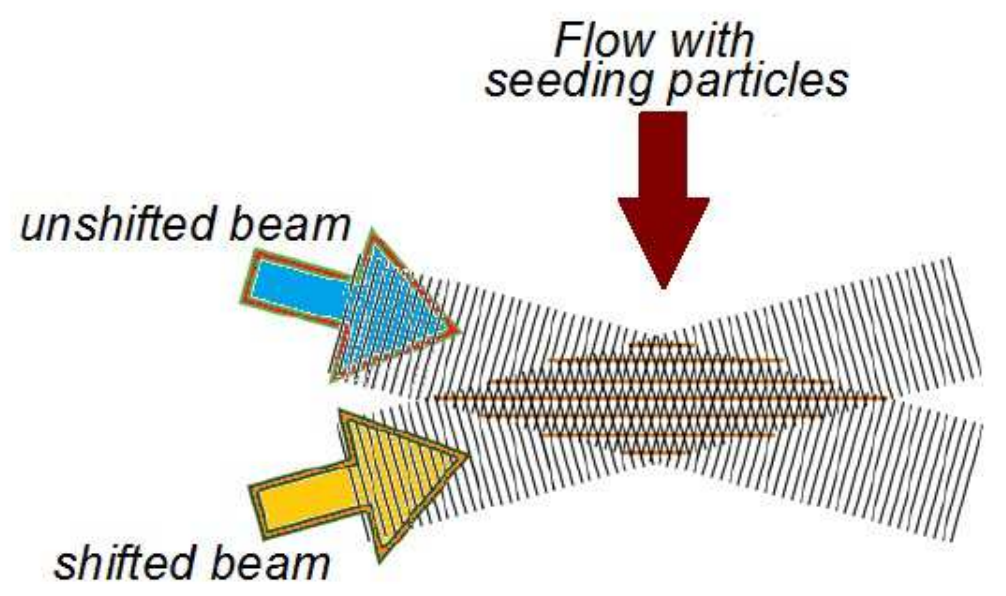

Figure 2.5: Measurement volume from intersecting coherent laser beams.

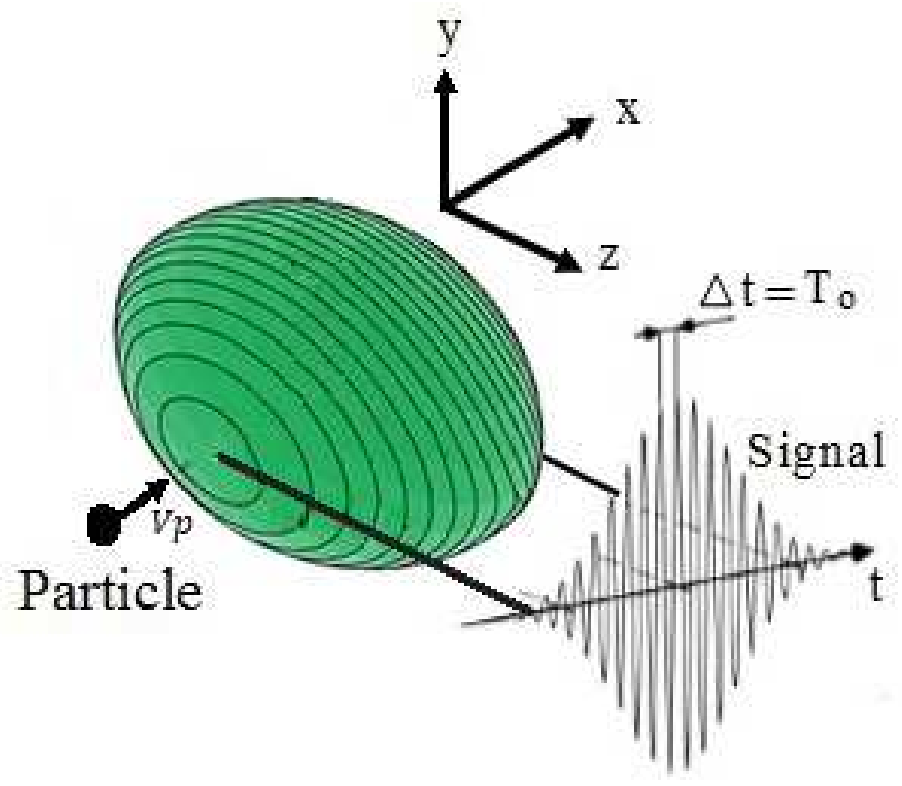

Figure 2.6: The formation of the measurement volume. Adapted from [72]. 
The light source that is used for this technique is a mono-chromatic laser light. This light is passed through a beam splitter, which is a mirror. This process will split the laser into two beams of equal intensity with different frequencies: one un-shifted, while the other is shifted. This process is done by passing the beam through a Bragg cell, which is composed of an electro mechanical transducer driven by an oscillator. A schematic diagram of a Laser Doppler Anemometry system can be seen in Figure 2.7.

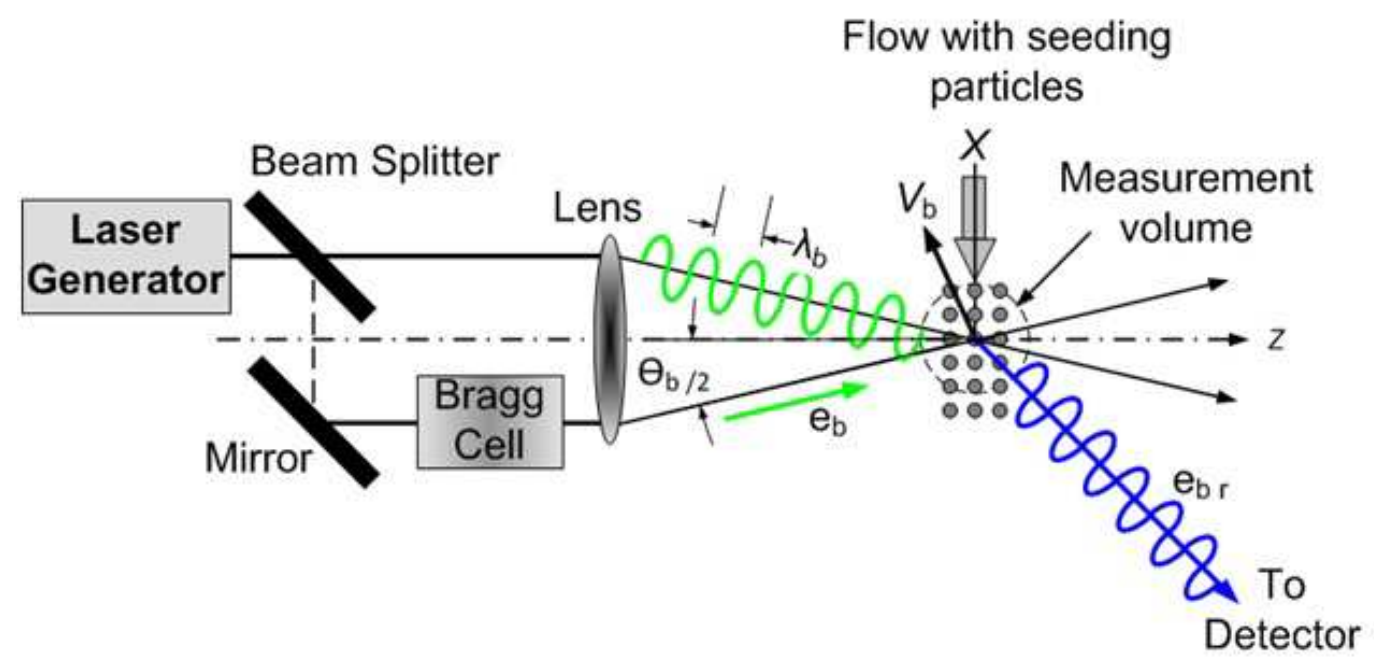

Figure 2.7: Block diagram of Laser Doppler Anemometry system. Adapted from [72].

The beams are then focused into the optical fibers that are attached to a probe (the transmitter). In the transmitter, the parallel beams that exit from the probe intersect in the measurement volume. The light that is scattered by the particles passing through the interference region is then collected by the receiving lens and is focused on the photo-detector. The interference of the two crossing beams in the measurement volume and the interference of the two scattering waves on the detector are contained in the scattered field due to the Doppler Effect [72].

As a particle passes through the measurement volume, a collision occurs with the incident light beams. The particle's velocity is then measured by using the Doppler Effect theory. These beams are described by a wavelength of $\lambda_{b}$ and velocity $e_{b}$, and the moving particle which has a velocity $V_{p}$. The light then scatters and is emitted 
in all directions at a variety of different angles. If a receiver, which is in this case the photodetector, is placed at an angle $\theta_{r}$, then the emitted scattered light from the particle to the photodetector will have a velocity of $e_{p r}$. This emitted light generates a sinusoidal signal with a frequency $f_{r}$. The frequency of the scattered light can be expressed using the following equation:

$$
f_{r}=f_{b}+\frac{V_{p}\left(e_{p r}-e_{b}\right)}{\lambda_{b}}
$$

This equation is valid for only one light beam. However, in recent LDA configurations the system uses the two intersecting incident beams, which allows the Doppler frequency, $f_{D}$, to be independent from the detector position [74]. Figure 2.8 illustrates a modern LDA arrangement, where $e_{1}$ and $e_{2}$ are the incident coherent beams that intersect with angle of of $\phi$. Therefore, the frequencies of the scattered lights are different and they can be expressed as follows:

$$
\begin{gathered}
f_{1}=f_{b 1}+\frac{V_{p}\left(e_{p r}-e_{1}\right)}{\lambda_{b}} \\
f_{2}=f_{b 2}+\frac{V_{p}\left(e_{p r}-e_{2}\right)}{\lambda_{b}}
\end{gathered}
$$

where, $f_{b 1}$ and $f_{b 2}$ are the frequencies of the incident beams.

The particle velocity calculation is based on the plane wave theory, that where any particle crosses the measurement volume, the scattered light intensity is strongest in the bright fringes and weakest in the dark fringes. The scattered light is measured, producing a modulation signal with a Doppler shift, which is directly proportional to the particle velocity component perpendicular to the angular bisector of the incident beams as [76]: 


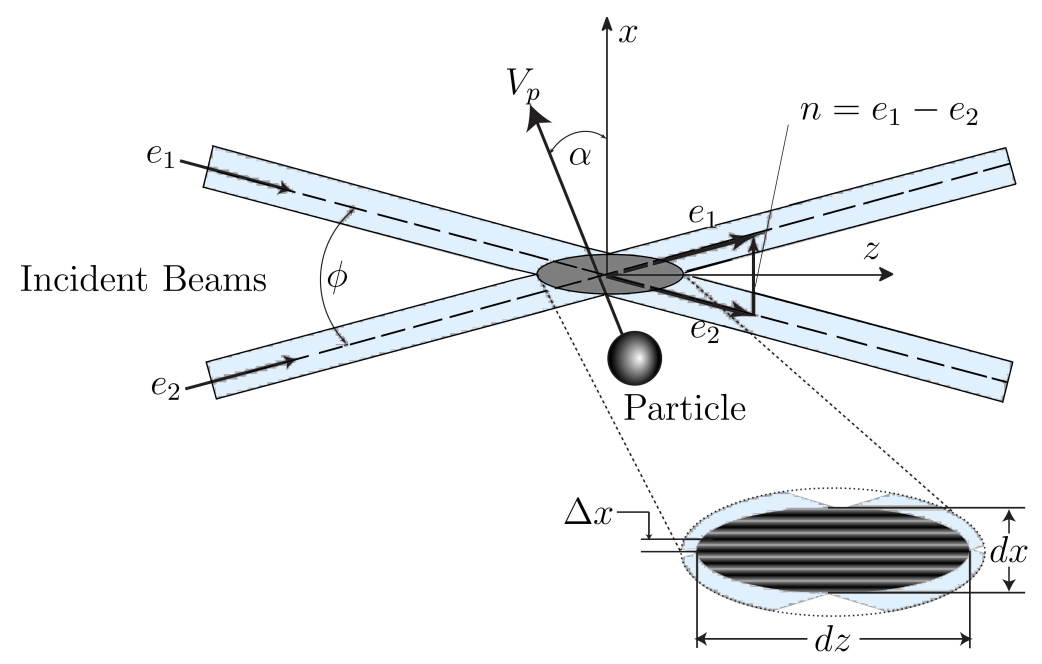

Figure 2.8: Modern two beam LDA configuration. Adapted from [79].

$$
\begin{gathered}
f_{D}=f_{2}-f_{1} \\
f_{D}=\frac{2 \sin \left(\frac{\phi}{2}\right) v_{p \perp}}{\lambda_{b}} \Longleftrightarrow v_{p \perp}=\frac{1}{2} \frac{f_{D} \lambda_{b}}{\sin \left(\frac{\phi}{2}\right)}
\end{gathered}
$$

In LDA system, photomultipliers transform the fluctuations in the scattering intensity into an electrical signal of Doppler pulses. Then it passes to a signal processor to be filtered and amplified, and the Doppler shift is obtained from spectral analysis of the processed digital signal using Fast Fourier Transforms (FFT). Thus, the particle velocity component can be calculated using Equation (2.7).

\subsubsection{Directional Ambiguity}

One disadvantage can be found in the LDA system. The directional ambiguity arises when the system is measuring the frequency of the light intensity variation, which arises due to a particle crossing a series of equally spaced fringes. This phenomenon 
only happens in two cases. First, when a particle crosses from bottom to top with a velocity $-v_{x}$, it will generate an identical signal as a particle that crosses from top to bottom with a velocity $+v_{x}$, thus there is ambiguity in the direction of the measured velocity. Second, if the particle remains steady in the measurement volume, a Doppler burst signal will not be produced. In order to avoid these incidents from occurring, a frequency shift $f_{o}$ should be introduced in the path of one of the laser beams by the Bragg cell. For the un-shifted beam, the fringe pattern will move at a constant speed in the direction of the frequency shift $f_{o}$. The particle moving with the fringes will generate a Doppler burst signal of a higher frequency, while the particle moving against the fringes will generate a Doppler burst signal of a lower frequency. The shifted Doppler burst frequency will now be:

$$
f_{D}=\left|f_{o}+\frac{2 \sin \left(\frac{\phi}{2}\right)}{\lambda_{b}} v_{x}\right|
$$

\subsection{Phase Doppler Anemometry (PDA)}

Phase Doppler Anemometry (PDA) expands the capabilities of the LDA to the measurement of particle velocity as well as its size. Besides the velocity, the PDA can measure a particle size simultaneously within the range of $0.5 \mu \mathrm{m}$ to $2.5 \mathrm{~mm}$ [77]. In addition, this determination can be gained at specific points in the sprays. This method works specifically on single particles, thus allowing a high spatial resolution to be obtained with very high accuracy [78].

\subsubsection{Principles of operation}

The Phase Doppler measurement technique is based on the mechanism of light scattering by particles. This scattering mechanism comprises the reflection, refraction and 
diffraction. The Mie theory provides a complete and exact mathematical description of light scattering by small and spherical particles, which is based on the change of the incident planar wave into a sum of spherical waves over the particle. In order to find the solution, the wave equation must first be solved. A solution for the spherical waves is obtained by principle of superposition [79]. This method is based on a solution of Maxwell's equations, which can be found in the literature [80].

\subsubsection{Light-scattering by particles}

As mentioned previously, the Phase Doppler measurement technique is based on the mechanism of light scattering by particles. The most significant scattering modes of the PDA system are first-order reflection, along with first and second order refraction. There are also higher orders of refraction but due to the low intensity of light at these higher orders, they are rarely used. The most substantial factor in the refraction mode is the relative refractive index, $n_{\text {rel }}=n_{\text {particle }} / n_{\text {medium }}$, which is the ratio of the particle's refractive index and the refractive index of the surrounding medium.

The fundamental principles of reflection and refraction of the light by spherical particles are illustrated in Figure 2.9, where various modes of light scattering are shown from a water droplet with $n_{r e l}=1.33$ and $d_{p} \gg \lambda_{b}$. As the incident ray interacts with the particle at point $\mathrm{A}$, the beam is partially reflected at angle $\phi_{o}$, and the other fraction of the incident ray penetrates the droplet and then escapes at point B with angle $\phi_{1}$. This process is known as the first order refraction. At point $\mathrm{B}$ an internal refraction occurs, where the other fraction of the beam is reflected and escapes at point $\mathrm{C}$ with angle $\phi_{2}$. This is known as a second order refraction. 


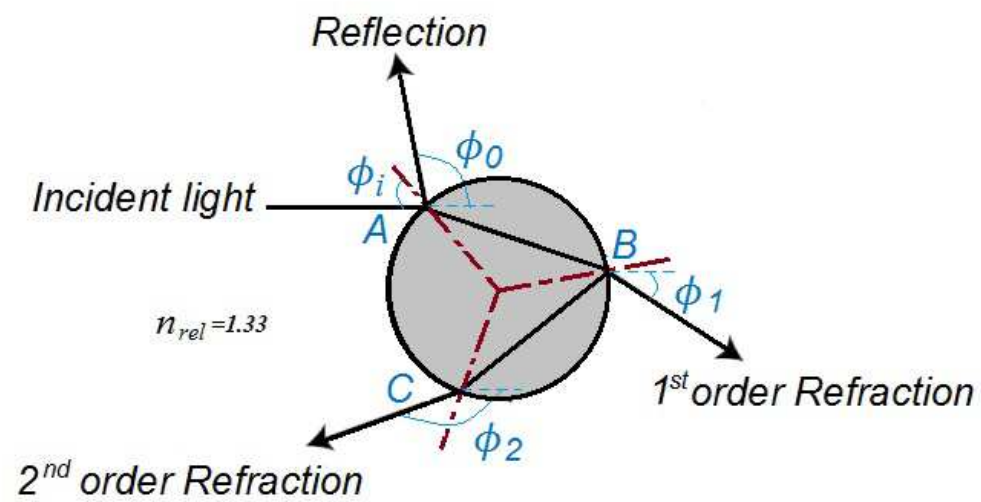

Figure 2.9: Light scattering by droplet of water, where $n_{r e l}=1.33$ and $d_{p} \gg \lambda_{b}$. Adapted from [83].

\subsubsection{Optical parameters of a Phase Doppler Measurement Sys- tem}

A typical optical arrangement of a two detector PDA system is shown in Figure 2.10. The principle of velocity measurement is the same as the one used in LDA. The velocity is determined by analyzing the frequency shift of light that is scattered by a particle flowing through the interference volume of at least two intersecting laser beams. Also, the determination of the droplet size is achieved by measuring the phase shift of the light encoded in the spatial variation of the fringes reaching the two detectors after traveling paths of different lengths through the droplets, as illustrated by cases A and B in Figure 2.12.

The phase shift is measured by two detectors, each of which examines the incoming light or the scattered light at spatially distinct portion of the collection lens. Furthermore, as in the configuration of modern PDA systems, there is more than one photo detector employed within a common housing. The phase difference between the signals generated by different detectors can therefore be measured. The phase difference is 


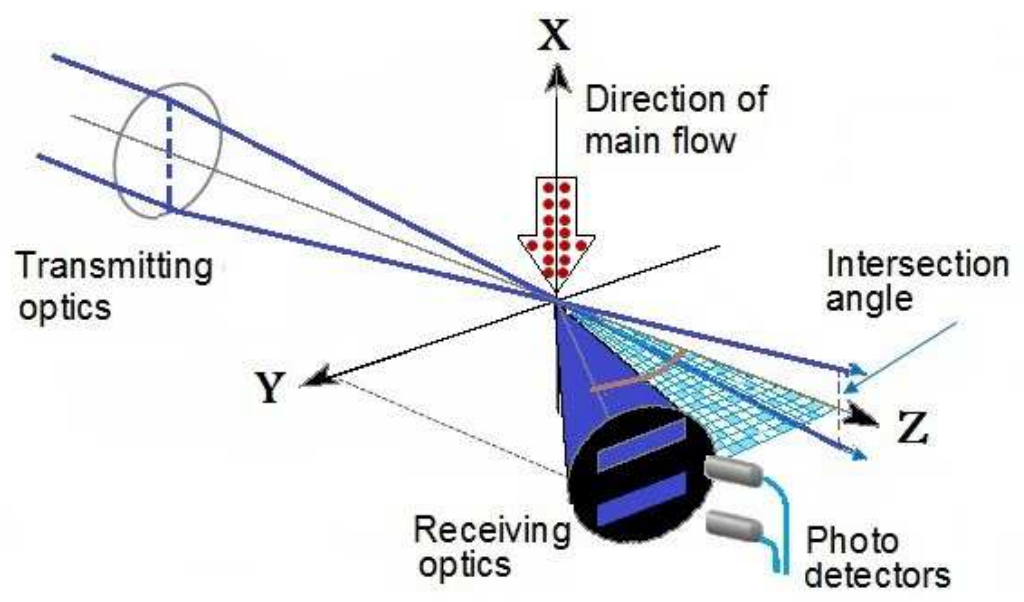

Figure 2.10: Optical arrangement of a two detector PDA system. Adapted from [81].

solely a function of the particle size. The phase at detector $i$ can be defined using the following expression:

$$
\Phi_{i}=\frac{\pi}{\lambda_{b}} D \beta_{i}
$$

Where $D$ is the particle diameter and $\beta_{i}$ is a detector geometrical factor which is a function of its spatial position, the scattering mode and the relative refraction index $n_{\text {rel }}$.

The phase difference, $\Phi$, is proportional to the size of the spherical particle. This relationship can be determined by the following expressions in two cases [81]:

a. Scattering is dominated by reflection:

$$
\Phi=\frac{2 \pi D}{\lambda_{b}} \frac{\sin \theta \sin \phi}{\sqrt{2(1-\cos \theta \cos \phi \cos \psi)}}
$$

b. Scattering is dominated by refraction:

$$
\Phi=\frac{2 \pi D}{\lambda_{b}} \frac{n_{r e l} \sin \theta \sin \phi}{\sqrt{2(1+\cos \theta \cos \phi \cos \psi)\left(1+n_{r e l}^{2}-n_{r e l} \sqrt{2(1+\cos \theta \cos \phi \cos \psi))}\right.}}
$$

Where: $n_{\text {rel }}=n_{\text {particle }} / n_{\text {medium }}$ refers to the relative refraction index. 
Figure 2.11 demonstrates the optical parameters of a PDA set-up, where the two incident beams intersect in the $x-y$ plane under the intersection angle $\phi$, the receiving systems are positioned in the $x-z$ plane at the scattering angle $\theta$ and the detectors are tilted against the $y-z$ plane under the elevation angle $\psi$. Since the phase difference $\Phi$ can be measured, and the wavelength $\lambda_{b}$, angles $\phi, \theta, \psi$ and relative refractive index $n_{r e l}$ are usually known, the particle diameter $D$ can therefore be calculated by using either (2.14) or (2.15) [78].

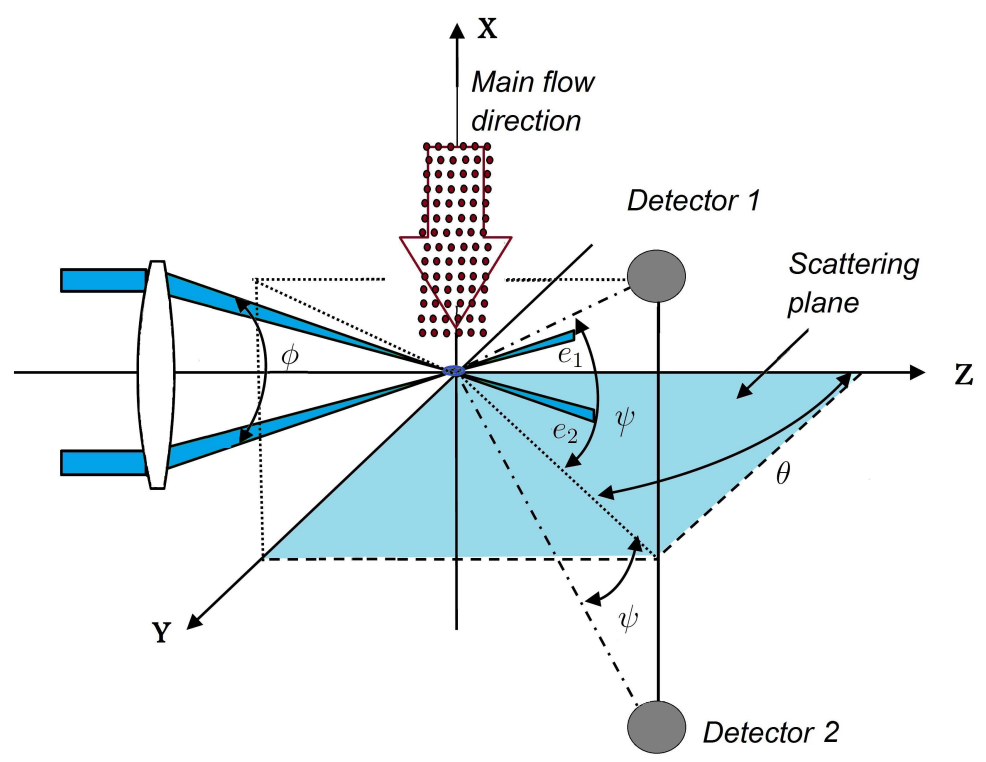

Figure 2.11: The Optical parameters of a PDA set-up. Adapted from [81].

A common problem appears if only two detectors are used in a case, where the phase difference angle, $\Phi$, is larger than $2 \pi \mathrm{rad}$, or $360^{\circ}$. In this case, an ambiguity in the measurement of the particle size occurs.. This phenomenon is called the " $2 \pi$ ambiguity". As shown in Figure $2.12 \mathrm{~B}$ and $\mathrm{C}$, the generation of the phase difference $\Phi$, for the small particle, which has a velocity $V_{2}$, would be the same as for the large particle, which has a velocity $V_{3}$. For example, in case $\mathrm{C}$ of Figure 2.8, if a large particle that causes a phase shift of $420^{\circ}$, it would be classified as a small particle with a phase 
shift of $60^{\circ}$ and the measured droplet diameter is $D_{3}^{\prime}$ which arises instead of the correct $D_{3}$. Their respective phase differences $\Phi$ are exactly out of phase by one period.

In order to avoid the ambiguity in the phase shift measurement and to diminish the measurement errors, a third detector, located close to one of the other two detectors, is typically employed in the PDA system, which is shown in Figure 2.13. As a result of such a configuration, a reduction in the phase shift of the signals between detector 1 and detector 3 is necessary. Thus, there is a reduction in the gradient of the phasediameter diagram, as illustrated in Figure 2.14. The phase shift, measured with $D_{1} / D_{3}$, determines the location of the particle's diameter in the measureable sections $(2 \pi$ jumps) of $D_{1} / D_{2}$. In addition, by using this technique a precise determination of the droplet diameter is gained with a large diameter range. An increase of the accuracy and high resolution are also obtained [78].
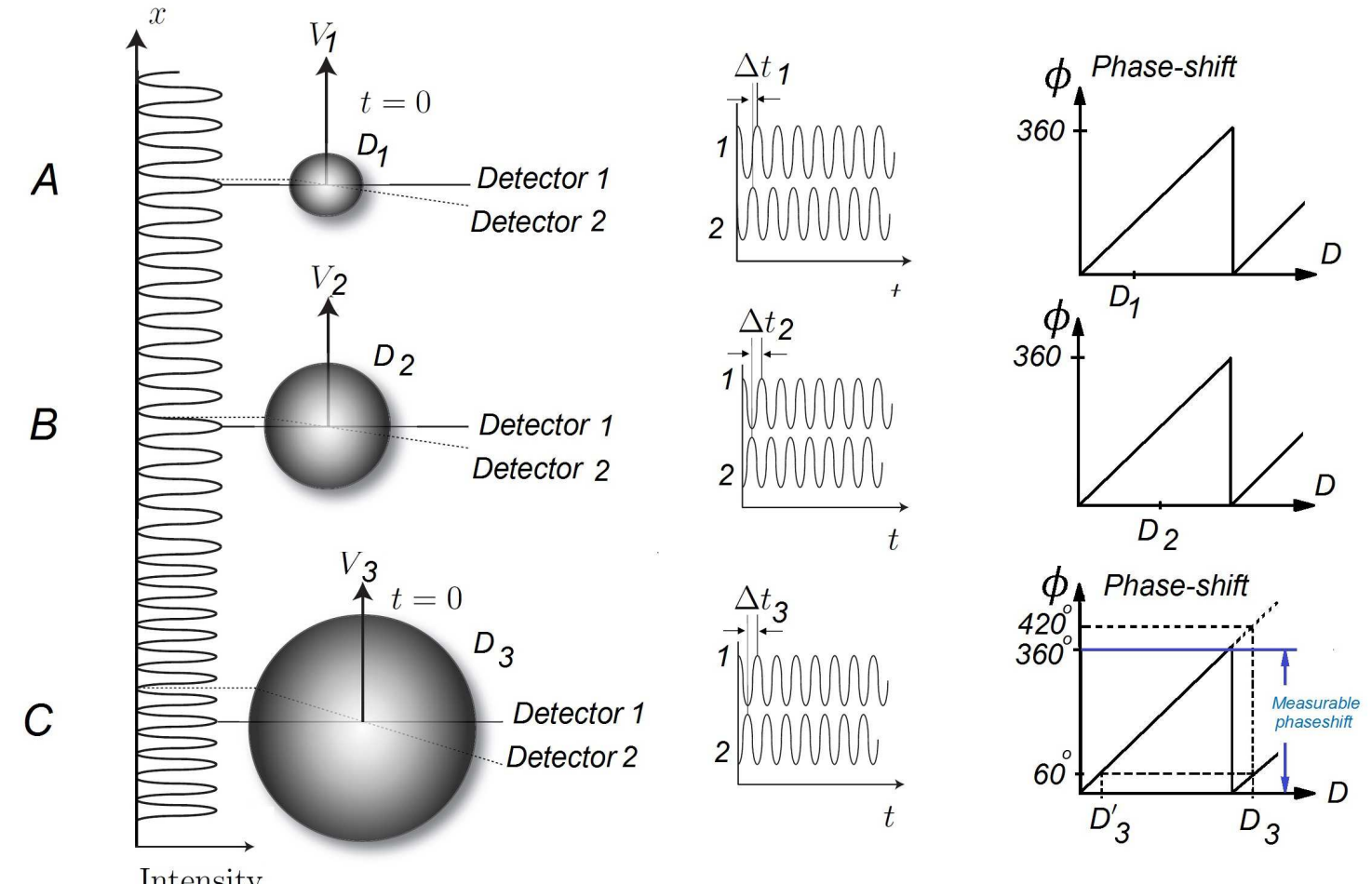

Figure 2.12: The phase shift between two detectors and the $2 \pi$ ambiguity. Adapted from [83]. 


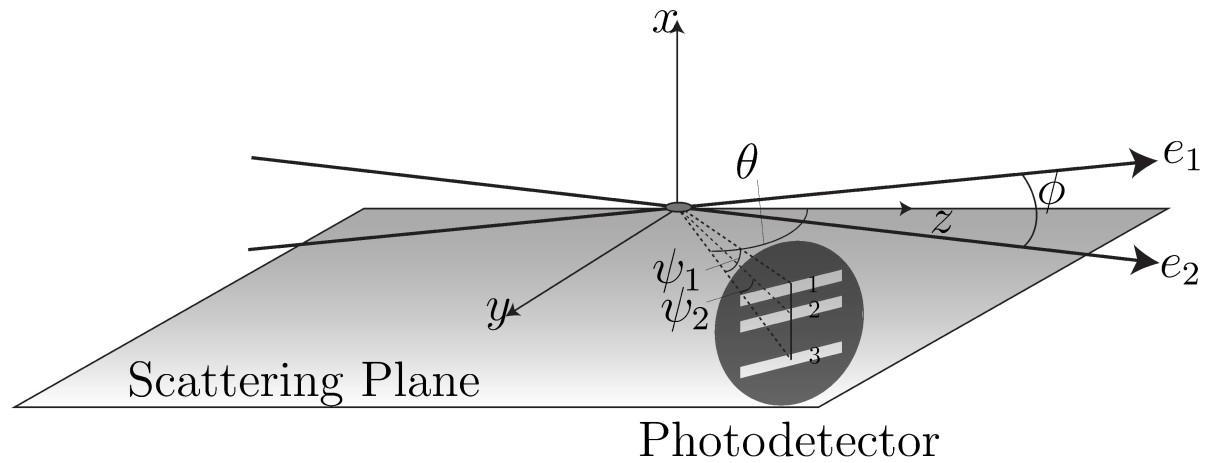

Figure 2.13: Modern PDA configuration. Adapted from [79].

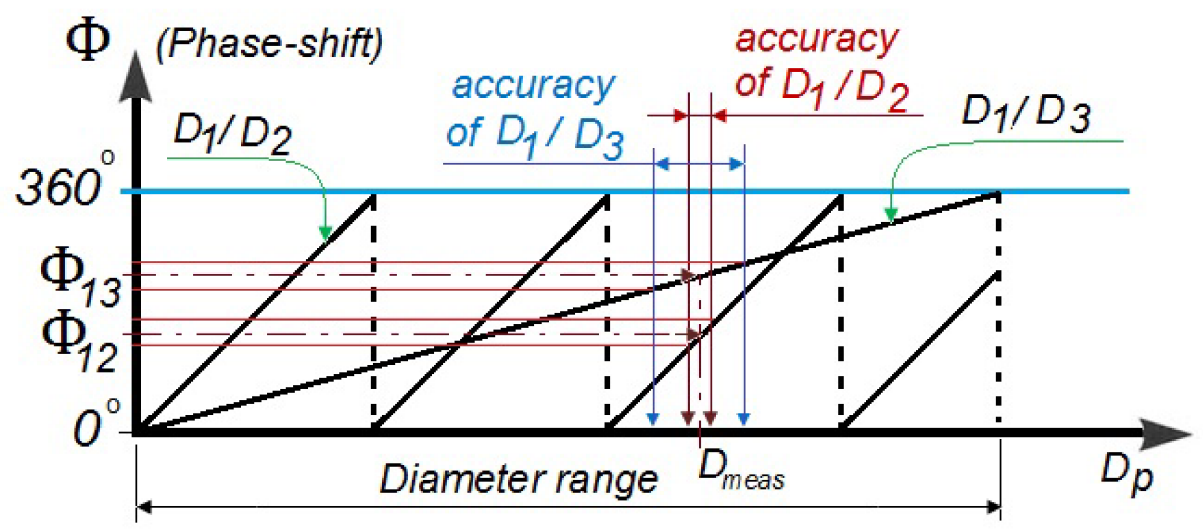

Figure 2.14: Extension of the diameter range in a three-detector PDA system. Adapted from [83]. 


\section{Chapter 3}

\section{Experimental Setup and Procedure}

\section{$3.1 \quad$ Objectives}

The main objective in the design and setup of the experimental apparatus, described in this section, is to make repeatable measurements of the spray issued from a pMDI and the Respimat by the PDA. As multiple measurements under similar conditions are required, the pMDI and the Spiriva Respimat inhaler is installed in a fixed position, to allow multiple actuations from a fixed reference position. The PDA system must also be installed and aligned in such a way, as to allow accurate measurement and reduce the uncertainty in the measurements. 


\subsection{Characterization of Aerosol Velocity and Size Dis- tribution by PDA}

\subsection{1 pMDI Apparatus}

A pneumatic cylinder mounted on a steel box beam is used to actuate the pMDI. The benefit of using this process is that the pMDI is activated consistently with constant speed and force. The cylinder is actuated by compressed air at a pressure of 3.5 bar, which is released into the cylinder by a solenoid valve to which, power is delivered via a mechanical relay (switch). Using such a controller will avoid the requirement for direct user interaction with the device for the triggering process, as can be seen in Figure 3.1.

In order to ensure that the pMDI will not move during the triggering process or between trials, the pMDI mount is bolted to an optical table. Also, to support the pMDI firmly, a negative moulded replica of the pMDI casing is manufactured in-house using Fillers (Bondo Automotive Body Fillers) as shown in Figure 3.2. This mould is afterwards glued to a $25 \mathrm{~mm}$ x $25 \mathrm{~mm}$ x $220 \mathrm{~mm}$ steel box beam. One end of the box beam is cut at an angle of $15^{\circ} \pm 0.25^{\circ}$ and welded to an $80 \mathrm{~mm} \times 80 \mathrm{~mm} \times 3.18 \mathrm{~mm}$ steel plate. To ensure accuracy, the manufacturing and the cutting of the angle is performed using HAAS VF2 CNC computer numerical control machine. The box beam and the steel plate are painted black to reduce the laser reflection. It is important for the apparatus to sit atop a rigid structure in order to minimize the vibration that might be introduced via the pneumatic system. In that light, the steel plate is bolted to the optical table. 


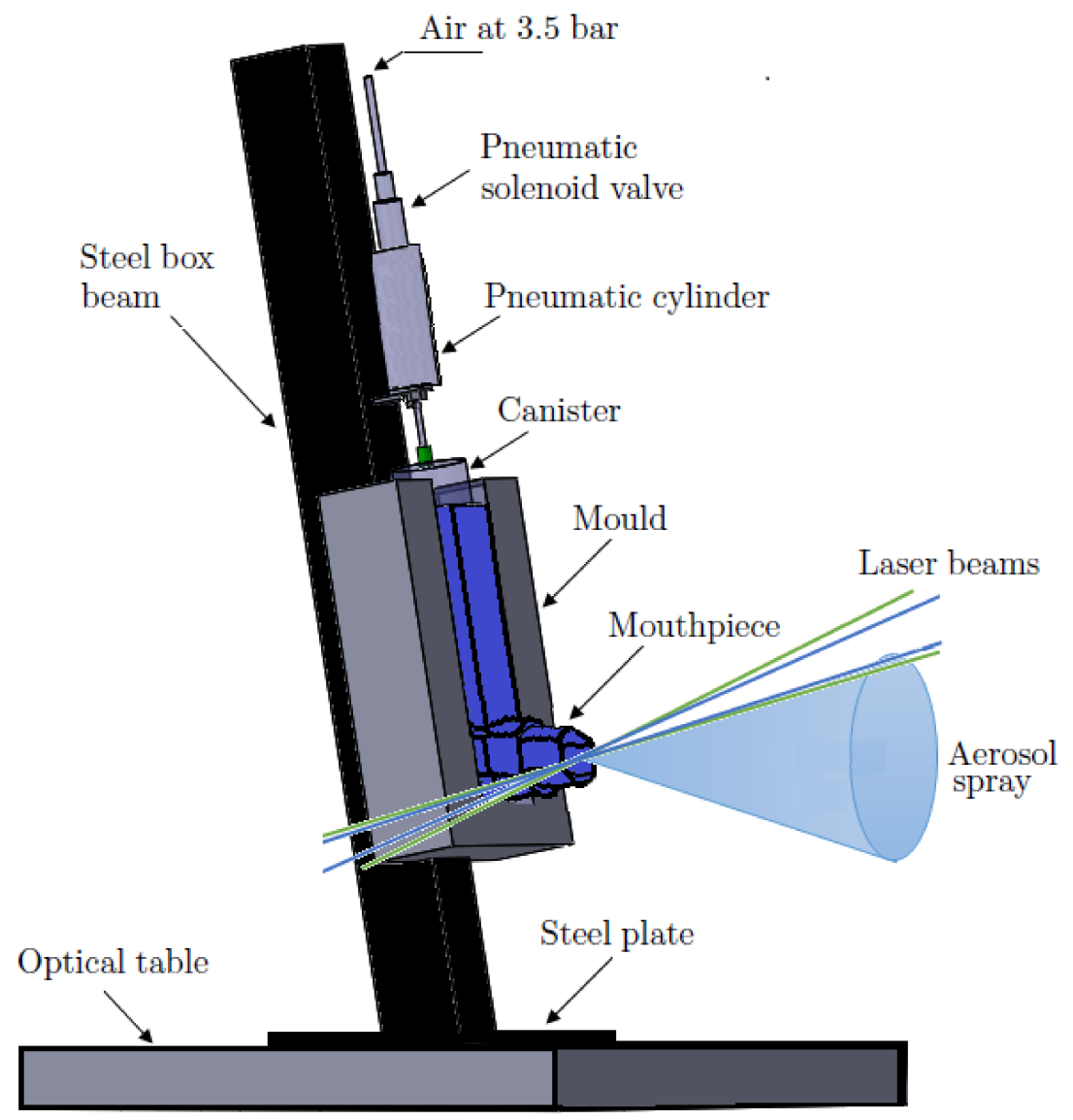

Figure 3.1: Actuation mechanism of the pMDI and the mounting apparatus. 


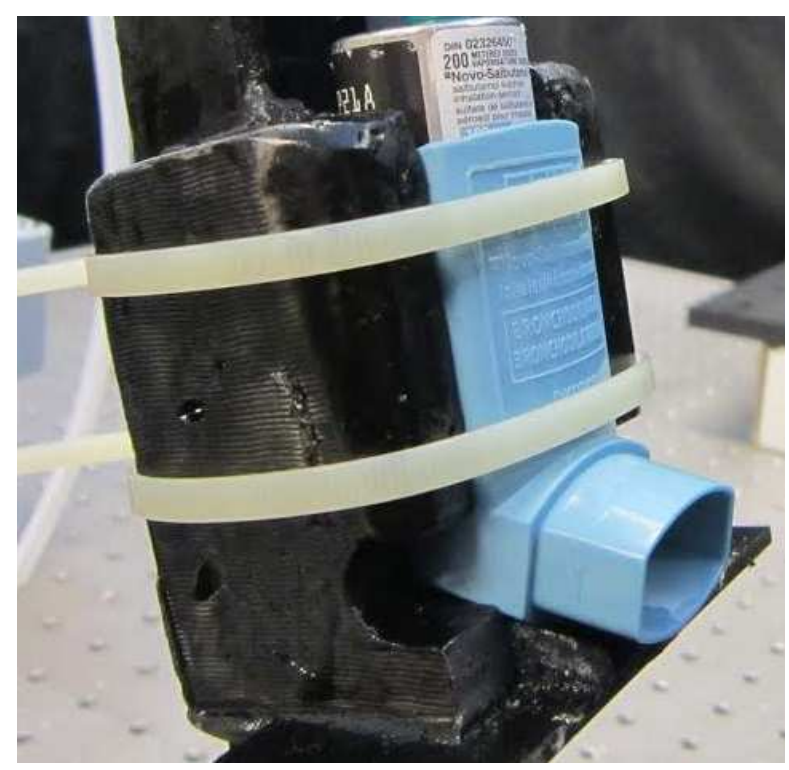

Figure 3.2: Photograph of the moulded replica of the pMDI casing.

\subsubsection{Custom Schmitt Trigger}

A Schmitt trigger is a comparator application with one input and one output. Under operation, the signal output can either be high or low, meaning that the output state depends on the input level, and will change only as input crosses a pre-defined threshold. If the output happens to be low, the output will not change to high unless the input signal goes above a certain upper threshold limit. Likewise, if the output happens to be high, the output will not change to low until the input signal goes below the certain lower threshold limit.

A Schmitt trigger circuit has been designed and constructed to synchronize the PDA system to the actuation event, by sending a TTL high pulse to the PDA system. In addition, this circuit compounds several sets of actuation data into a single master file in the BSA software, which can then be averaged. This information is necessary to characterize the spray in a time resolved manner. The trigger works as comparator, thus when the control switch is toggled a voltage signal of $5 \mathrm{~V}$ is generated from the pneumatic solenoid valve during the actuation procedure, which means that the pneu- 
matic solenoid valve is fully opened. The comparator is configured such that at $4.02 \mathrm{~V}$, at the instant where the control switch toggles, the trigger output changes from TTL low to high. This TTL pulse is sent via a BNC cable to the PDA system. This trigger allows multiple actuation events to be superimposed and can later be time averaged during post-processing.

The trigger circuit is composed of one 741 operational amplifier, one transistor and 5 resistors as illustrated in Figure 3.3. The generated signal transmits to the amplifier when the pneumatic solenoid valve is opened, where it compares the signal to a threshold voltage of $4.02 \mathrm{~V}$. Immediately after the pneumatic solenoid valve voltage surpasses the 4.02 $\mathrm{V}$ threshold, the output of the amplifier saturates the transistor, which switches the output from +5 to $0 \mathrm{~V}$.

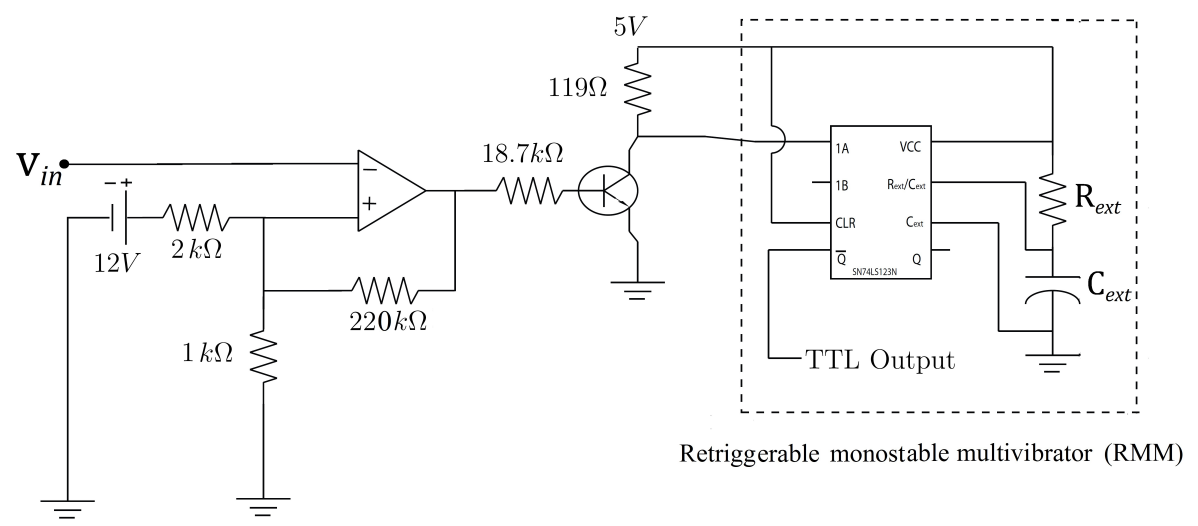

Figure 3.3: Schmitt trigger and monostable vibrator circuit.

It is found that there is a delay in the pneumatic cylinder response that could affect the data acquisition time. In order to prevent this from happening, a monostable multivibrator SN74LS123 chip set is used. This chip will keep a rising or falling edge signal high or low for an extended period of time. From Figure 3.3, the $C_{\text {ext }}$ and $R_{\text {ext }}$ values have been selected to provide a pulse width of $4000 \mathrm{~ms}$, which is longer than the actuation period of the spray. The pulse duration is calculated using Equation 3.1 
below.

$$
t_{w}=k \times R_{e x t} \times C_{e x t}
$$

Where : $k$ is a multiplifying factor equal to 0.45 .

$R_{\text {ext }}$ is an external timing resistance in $K \Omega$.

$C_{\text {ext }}$ is an external timing capacitor in $p F$.

$t_{w}$ is the output pulse width in $n s$.

\subsubsection{Spiriva Respimat Apparatus}

To support the Spiriva Respimat inhaler firmly, a negative shape of the space between the dose release button and safety catch, a replica of the Spiriva Respimat inhaler casing is manufactured using HAAS VF2 CNC (computer numerical control) machine. It is made of aluminium and the cap holder is used as support for the Spiriva Respimat inhaler by using a pin as a reference position in case the inhaler is changed. In order to ensure that the Spiriva Respimat inhaler will not move during the triggering process or between trials, the Spiriva Respimat inhaler mount is bolted to an optical table. This setup can be seen in Figure 3.4.

\subsubsection{Procedure}

The test procedure for the data acquisition at each measurement location is taken at ambient laboratory conditions after allowing some time for the apparatus to reach an equilibrated operating condition. Therefore, the average laboratory conditions for the temperature (the average room temperature is $22.5^{\circ} \pm 2 \mathrm{C}$ ) is recorded, as this condition might be affected by the heat generated from the laser system. The test is then be performed, to acquire the spray data by repeatedly actuating the device 


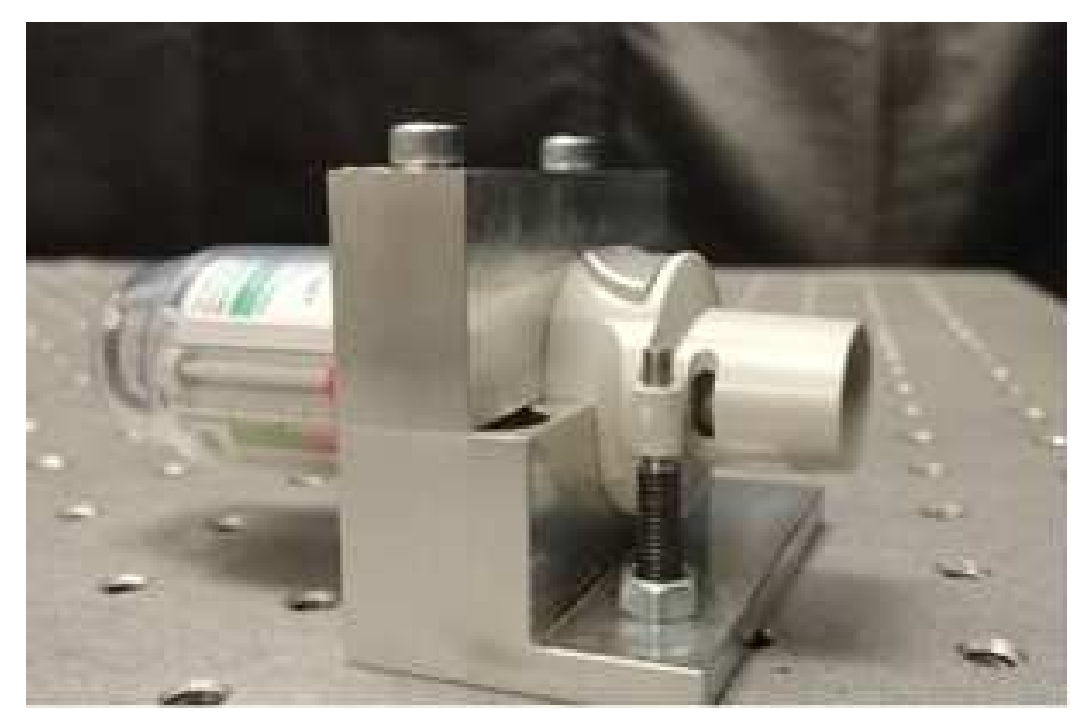

Figure 3.4: The mounting apparatus of the Spiriva Respimat.

at each measurement station. Between five and fifteen actuations are carried out at each station. A minimum number of five sprays has been chosen to accomplish a representative ensemble of mean measurement values.

In pMDIs measurements, to avoid the stem block from overcooling, a time period of approximately 10 seconds is allowed between each actuation, otherwise ice could form and that would block the actuator orifice. In addition, the pMDI canister is primed by first shaking the device and then actuating it once by releasing the spray in open air. This process is performed before administering the drug, as is recommended in the pMDI instruction leaflet.

To characterise the spray spatially, stations are selected to take discrete measurement at several locations in the flow field. The distance from the nozzle orifice to the mouthpiece edge of the all tested pMDIs is $25 \mathrm{~mm}$. Therefore, the mouthpiece edge is the origin of the measurements, and the measurements are taken at different increments of the axial distance from the origin. The first axial measurement location is defined as $0 \mathrm{~mm}$. The measurements stations positions are given in a cylindrical polar coordinate system $(x, r, \theta)$, which can define three spaced axial stations, with three radial locations 
intersected at an angle of $45^{\circ}$. Consequently, four cylindrical measurement planes are defined at each axial station, $0^{\circ}, 90^{\circ}, 45^{\circ}$ and $-45^{\circ}$. Figure 3.5 shows the details of these locations.

Regarding the Spiriva Respimat inhaler measurement, the distance from the nozzle orifice to the mouthpiece edge is $6.5 \mathrm{~mm}$. A single point measurement is taken at this location $(-18.5,0)$ for the Spiriva Respimat only. To compare all tested inhalers (pMDIs and SMI) at the same locations the distance of $25 \mathrm{~mm}$ from the nozzle orifice is set as the first of three axial stations selected for measurement defined as $x=0 \mathrm{~mm}$, and the measurements are taken at three different increments (axial distance) from the origin as in the pMDI test, which illustrated in Figure 3.5. In this case, the Spiriva Respimat inhaler is actuated manually and each test is taken as one measurement. Also, only two cylindrical measurement planes are defined at each axial station which are the vertical and the horizontal axes. In addition, a time period of approximately one minute is allowed between each actuation.

The PDA system that is used in this study is provided by Dantec Dynamics. This system is attached with a $2 D$ traversing support system which moves the transmitter and receiver; allowing multiple measurement points to be investigated without realigning the optics.

Figures 3.6 and 3.7 show the mentioned system, where the transmitting optics consist of a Spectra-Physics Stabilite 2017 laser which generates a multiline argon-ion laser beam with a maximum power output of roughly $2 W$. In the transmission case a quartz prism splits the incoming beam when it passes through transmission box into two lines of $514.5 \mathrm{~nm}$ and two lines of $488.0 \mathrm{~nm}$. By using fiber optics, both of the lines are connected to a FiberFlow $60 \mathrm{~mm}$ 2D transmitter. The receiving optics use a Hidense FiberPDA 57 X50 receiver.

The FiberPDA Box is coupled with the BSA P60 Flow and Particle Processor. Also, the FiberPDA box is connected to the receiving optics using optical fiber. In 

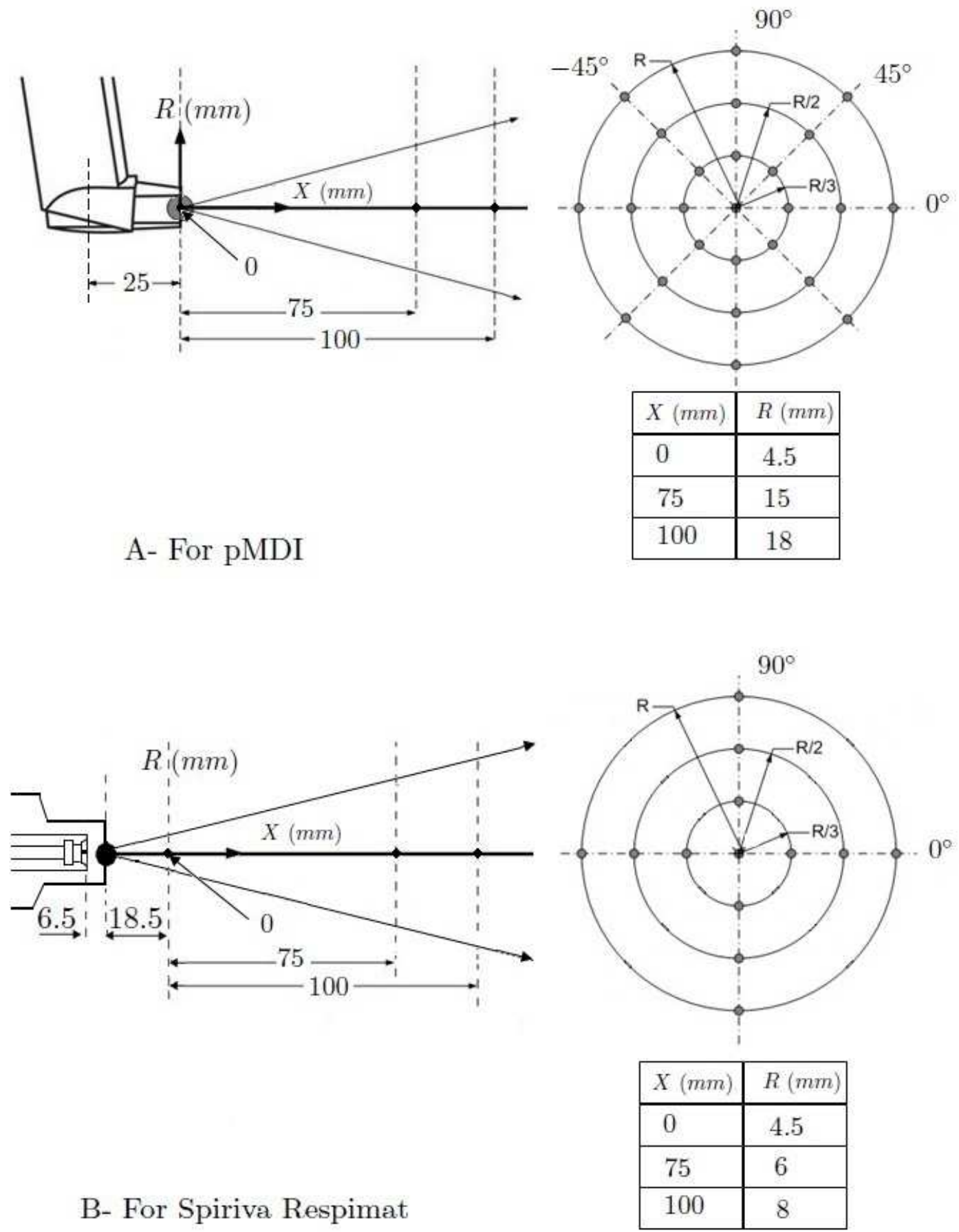

Figure 3.5: Experimental measurement locations. 


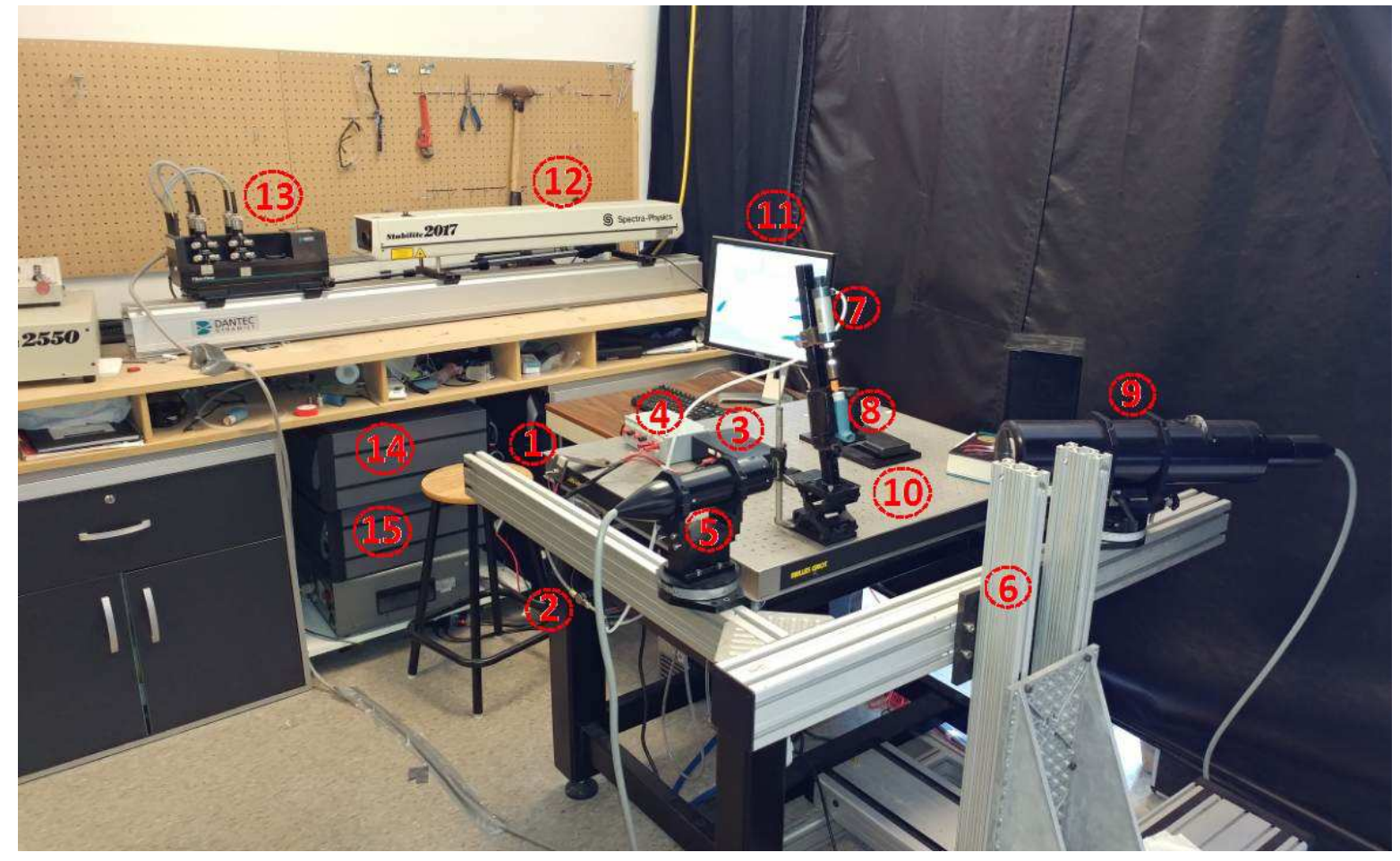

Figure 3.6: PDA system and experimental apparatus.

1. Control switch

2. Pneumatic solenoid valve

3. Power Supply

4. Integrated trigger circuit

5. $60 \mathrm{~mm} \mathrm{2D}$ Transmitting Probe

6. 2D Traverse System

7. Pneumatic cylinder

8. The pMDI
9. HiDense FiberPDA Receiver

10. Optical table

11. Desktop software

12. Laser generator

13. Beam splitter

14. Fiber PDA Detector

15. BSA P60 Processor 
order to convert the optical Doppler burst into a current, the FiberPDA box contains 3

photomultiplier tubes. Subsequently, the analysis of the frequency content of the signal is done via Fast Fourier Transforms (FFT) when the current is sent to the BSA P60 processor. The processor then sends the result files to the post-processing software on a desktop computer.

In Spiriva Respimat inhaler measurements only 1-PDA is used, instead of the 2-PDA mentioned system which is due to the weakness in the blue laser beam $(488.00 \mathrm{~nm})$. Therefore, the green laser beam of $514.50 \mathrm{~nm}$ of the 1-PDA measures the particle size in the vertical plane, then the transmitter is rotated by 90 degrees to measure the velocity in the horizontal plane.

\subsubsection{Summary of Process Diagram}

The experimental process diagram is illustrated in Figure 3.7. The procedure of the actuation is for the pMDI only, whereas the Spiriva Respimat inhaler is actuated manually not with the pneumatic cylinder. Therefore, the data is collected in the same sequence. The sequence of events leading to the acquisition of the data is as follows:

1. A control switch is toggled, delivering power to the pneumatic solenoid valve.

2. The pneumatic solenoid valve toggles, delivering air at 3.5 bar to the pneumatic cylinder.

3. The pneumatic cylinder extends, placing force on the pMDI canister.

4. The pMDI canister is depressed.

5. The FiberPDA detects the optical Doppler burst of spray droplets as they cross the measurement volume. 
6. The FiberPDA sends a current proportional to the burst intensity to the BSA processor.

7. The BSA processor analyzes the frequency content of the burst and calculates the droplet velocity and diameter as a function of time following the trigger signal.

8. The results are then sent to the BSA Flow Software.

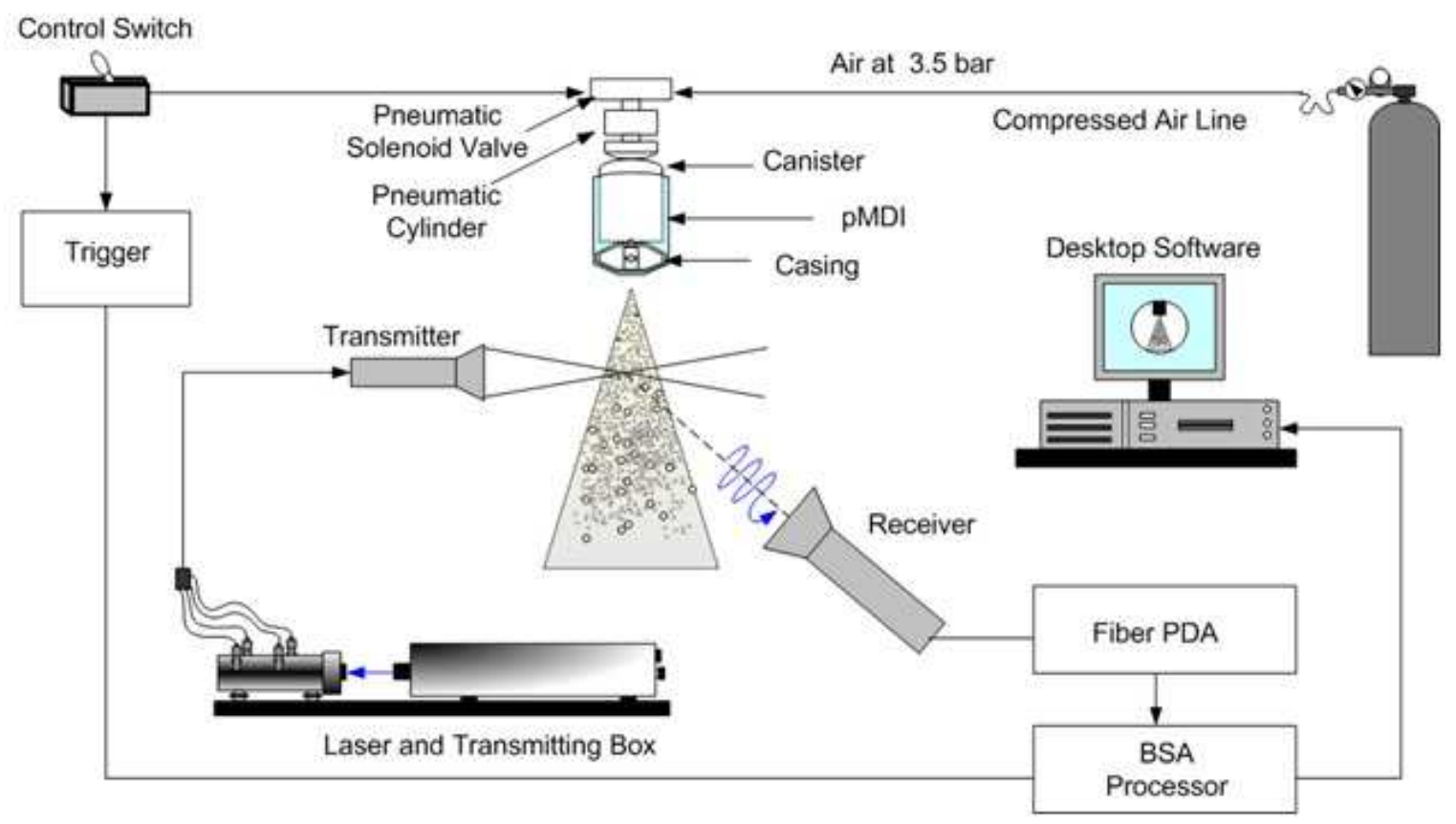

Figure 3.7: Experimental process diagram.

Once the data collection is completed, each spatial location from the PDA system is extracted from the BSA Flow Software as a text file, which is subsequently processed as an input to a MATLAB file to complete the data processing. Four commercial salbutamol sulfate pMDI drug products are tested in this study, namely: Apo-Salvent CFC Free, Airomir HFA, Novo- Salbutamol HFA, and Ventolin HFA. All of tested pMDI are HFA based suspensions. In addition to one Spiriva Respimat SMI used in this study, contains $2.5 \mu \mathrm{g}$ of tiotropium bromide monohydrate, per puff. 


\subsubsection{Selection of Instrument Parameters}

\subsubsection{Laser Power}

Due to a limitation of the laser's power source, the system's capability is restricted to a maximum of $2 \mathrm{~W}$. The accuracy of the measurement heavily depends on the laser power parameter. During the measurement, the beam attenuates through the spray droplet cloud that surrounds the measurement volume, which may cause some difficulties in the investigations. Also, in the low laser power region the small particles are not going to be detected. Furthermore, it was previously reported that at low laser power levels, the possibility of a biased high droplet size statistic is accrued [82]. Therefore, the laser power must be set such that the Doppler burst caused by droplets surpasses the burst threshold. In addition, the experiments are conducted at $0 \mathrm{~mm}$ distance from the mouthpiece axis, and the system is tested in the range of $0.1 W$ to $2 W$ in order to see the effect of these power parameters on the particles that are measured by the software for both droplet diameter and velocity. Figure 3.8 illustrates the dependency of the average droplet diameter on laser power for Novo- Salbutamol HFA. Similarly, the laser power effect on the average droplet velocity is showing in Figure 3.9.

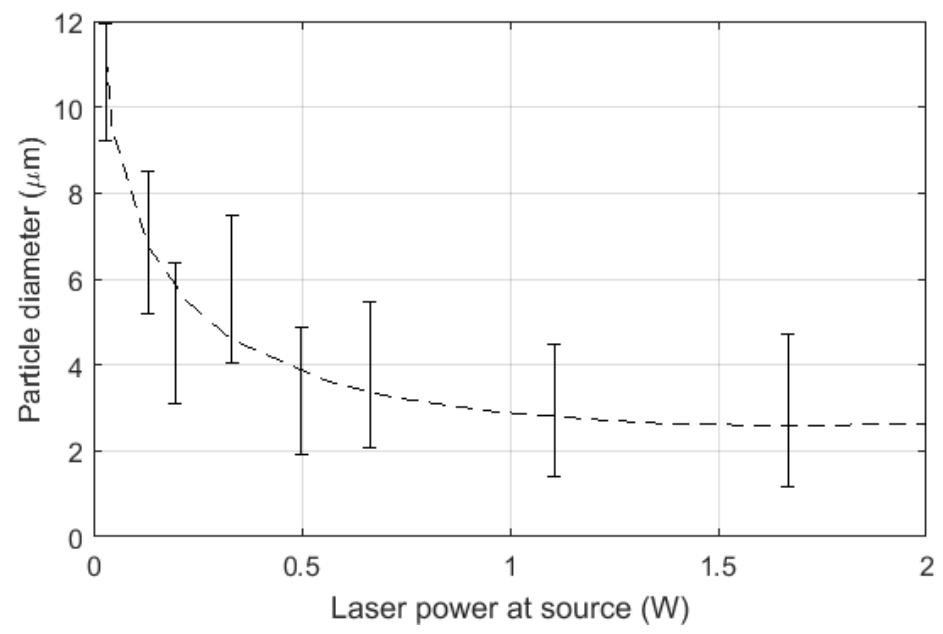

Figure 3.8: Droplet diameter dependency on incident laser power. 


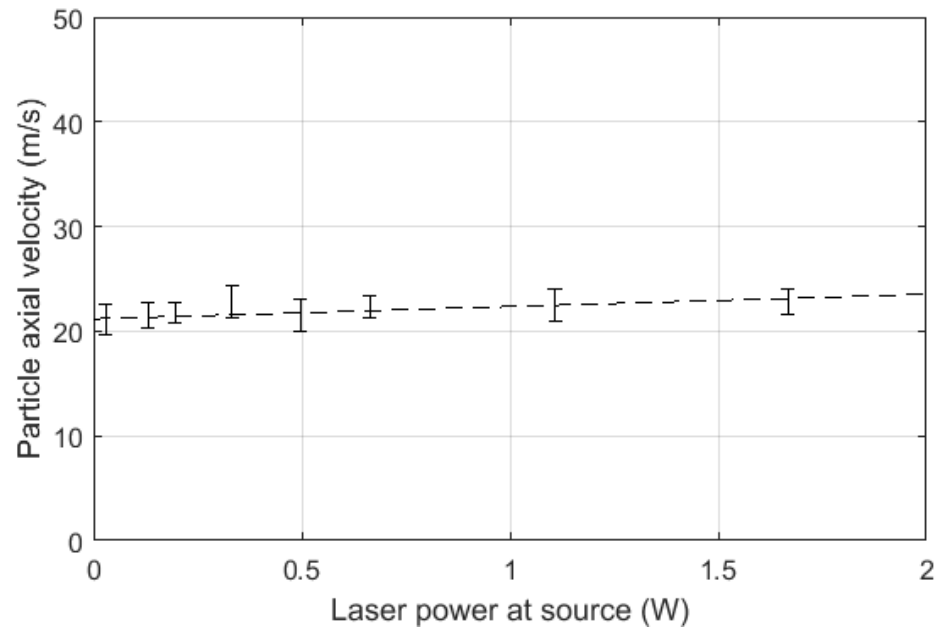

Figure 3.9: Droplet velocity dependency on incident laser power.

\subsubsection{High Voltage (HV), and Signal to Noise Ratio (SNR)}

Similarly, the HV affects the data acquired by the photodetector. In the present system, in each of the detectors, the $\mathrm{HV}$ is varied from $500 \mathrm{~V}$ up to $1400 \mathrm{~V}$. An increase of $\mathrm{HV}$ to very high levels will lead to the combination of unwanted noise to the signal. Consequently, the HV is usually set simultaneously with the signal to noise ratio (SNR). The data rate is the determining factor in this situation, as an increase in the data rate usually represents a better acquisition, unless too much noise is validated by droplets [82]. Another criterion that is used for the selection of the HV is the anode current, which must be similar for all of the detectors. Therefore, to find the best data rate and the overall time resolved velocity, a series of tests are carried out to determined the best HV and gain for the detectors. It is determined that the LDA1 channel required an $\mathrm{HV}$ of $1000 \mathrm{~V}$ with a gain of $24 \mathrm{~dB}$ and the LDA4 channel required an $\mathrm{HV}$ of $1200 \mathrm{~V}$ with a gain of $24 d B$ for optimal data acquisition conditions.

\subsubsection{Transmitter Optic Aperture}

The PDA measurement capabilities are determined by the components of the system. For instance, the location of the laser beams' intersection, and the range of droplet 
velocities and sizes can be determined by the PDA transmitter. Nevertheless, by changing parameters such as the focal length for the transmitting optics, the measurement volume also, can be altered. In this study, only one transmission optic is used with a $160 \mathrm{~mm}$ focal length lens (over the available $400 \mathrm{~mm}$ focal length lens), as the measurement volume must be kept at small as possible for dense sprays, and the $160 \mathrm{~mm}$ produces the smallest ellipsoid volume resulting in a measurement volume which has the largest energy density. The focal length of the $160 \mathrm{~mm}$ lens produces an intersection half angle $\phi / 2=6.772^{\circ}$. Knowing the incident beam diameter $d_{b}=0.0766 \mathrm{~mm}$, the size of the two ellipsoid volume is then approximated by Equation (3.2).

$$
\delta_{x}=\frac{d_{b}}{\sin \left(\frac{\phi}{2}\right)} \quad \delta_{z}=\frac{d_{b}}{\cos \left(\frac{\phi}{2}\right)} \quad \delta_{y} \simeq d_{b}
$$

The resulting ellipsoid volume is $\left(\delta_{x}=0.0782, \delta_{y}=0.0766\right.$, and $\left.\delta_{z}=0.658 \mathrm{~mm}\right)$.

\subsubsection{Receiver Optic Aperture}

The receiving optics used in this study employs a Hidense FiberPDA 57 X50 receiver. The optic is a Dantec receiver, which has an aperture slit located in front of its three photodetectors. In order to validate the Doppler burst, the PDA is restricted to a single particle in the measurement volume. Thus, to reduce this phenomenon in the high dense flow, a slit is employed by the PDA receiver, which then reduces the measurement volume in the $z$-direction. The controller of the slit can vary by $25,50,100$, and $200 \mu m$. In the case where there is more than one particle and their sizes are comparatively small to the measurement volume, they could trigger the Doppler burst threshold. The slit is graphically depicted in Figure 3.10, which shows the possibility of the measurement in dense sprays where the droplet proximity is high. The mechanism of the slit works as follows, the light gray ellipsoid indicates a measurement volume where several particles can be identified at time, $t$. The receiver is placed at an angle of $\theta=30^{\circ}$ and the first-order refraction is the dominant scattering mode of these particles. In which case, 
the first-order refraction of each particle is measured, except for droplet A which is impeded by the slit. The reflection of droplet B is also recognised, nevertheless, knowing the scattering properties discussed earlier and setting $\theta$ at $30^{\circ}$, the intensity of the Doppler burst of the reflected droplet is orders of magnitude weaker than the refracted light. The result will be the validation of droplet A only. However, as the aperture is reduced in size, there is a reduction in light collection efficiency and the laser power or signal gain should be increased to compensate, a small droplet may be well below the detection threshold. Therefore, an increase in laser power would decrease the validation rate and generate incorrect data.

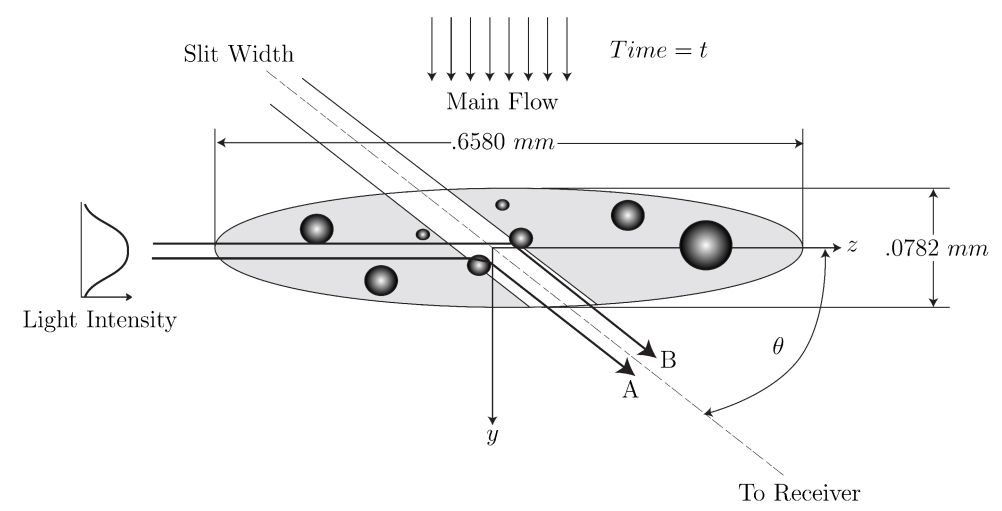

Figure 3.10: The mechanism of the slit aperture for Gaussian intensity distributed measurement volumes. Adapted from [78].

The azimuth angle $\psi_{i}$ between the three photodetectors inside the receiving optics is controlled by the focal length of the receiving optics, which also, controls the size range of the measured droplets. However, droplet size statistics measured using a PDA are not available for the pMDIs. Hence, the available $310 \mathrm{~mm}$ focal length receiving lens is used as the capable size range of this lens is from $0 \mu \mathrm{m}$ to $156.5 \mu \mathrm{m}$.

Additional, adjustment that enables a more precise control of the azimuth angle is the aperture mask positioned between the receiving lens and segmented lens within the receiver. For the optical configuration selected above, the size range can further be controlled by the mask to the values summarized in Table 3.1 [83]. 
Table 3.1: Maximum droplet size vs mask selection.

\begin{tabular}{|c|c|c|}
\hline Mask A & Mask B & Mask C \\
\hline $0.1-40.43 \mu \mathrm{m}$ & $0.1-65.66 \mu \mathrm{m}$ & $0.1-156.50 \mu \mathrm{m}$ \\
\hline
\end{tabular}

\subsubsection{Processor Limitations}

Due to the available lens that has a focal length of $160 \mathrm{~mm}$, the maximum measurable velocity bandwidth is limited to $62 \mathrm{~m} / \mathrm{s}$. It is found that the Ventolin HFA brand has a higher velocity than this. This would lead the processor to be upgraded to allow a larger measurement bandwidth. However, to expand the measurement bandwidth and solve the processor limitation it is necessary to adjust the digital filtering frequency. This frequency can be adjusted such that the central velocity is set to 32 $\mathrm{m} / \mathrm{s}$, thus measuring velocities from $0 \mathrm{~m} / \mathrm{s}$ to $62 \mathrm{~m} / \mathrm{s}$ as illustrated in Figure 3.10.a. This method is used for all brands. Just for the Ventolin HFA only, to measure the particles that have larger velocities, the measurement is repeated at the same station through adjusting the frequency such that the central velocity is now set to $93 \mathrm{~m} / \mathrm{s}$ to be able to measure large velocities from $62 \mathrm{~m} / \mathrm{s}$ to $134 \mathrm{~m} / \mathrm{s}$, as shown in Figure 3.10.b. Subsequently, the data can be superimposed and analyzed producing the whole emitted particles from the puffer; even those with high velocities that exceeded the processor's limitation. Figure 3.11.c demonstrates how the time resolved velocity data fits after 


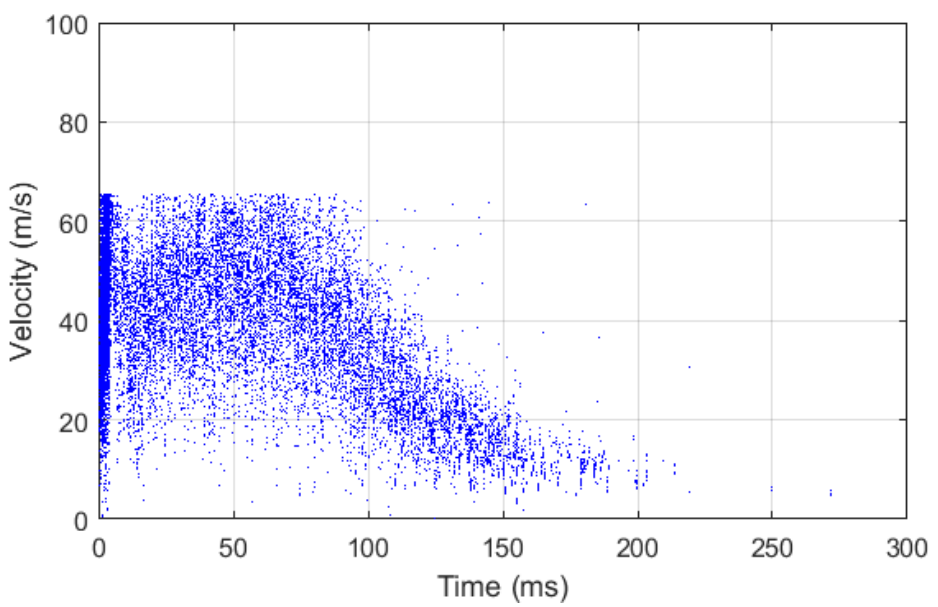

(a) Central velocity at $32 \mathrm{~m} / \mathrm{s}$.

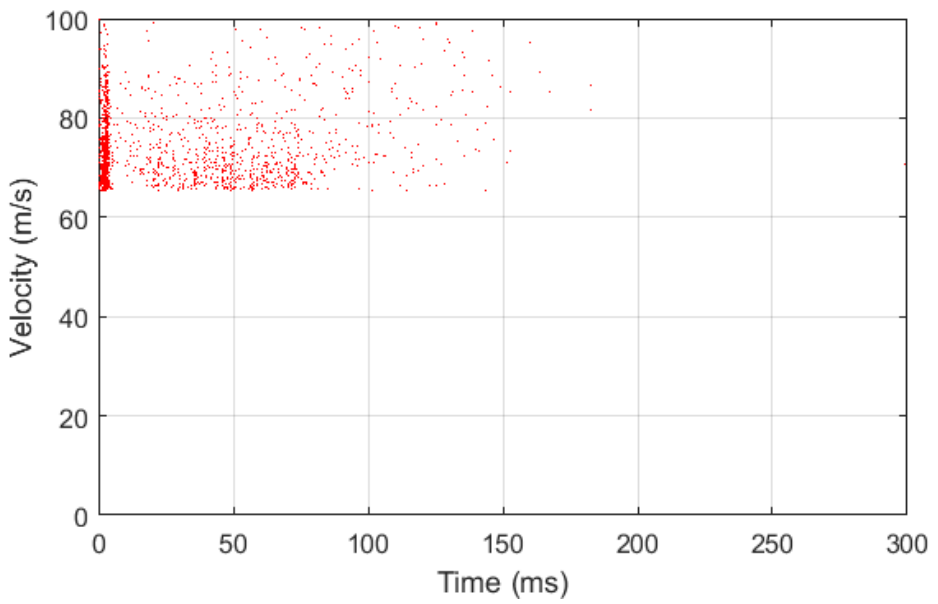

(b) Central velocity at $90 \mathrm{~m} / \mathrm{s}$.

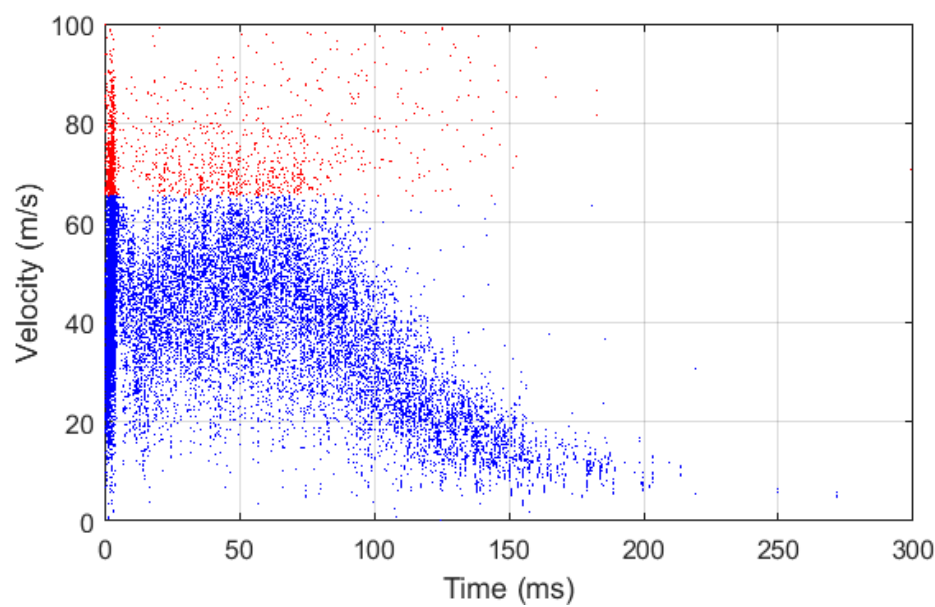

(c) Coupling data

Figure 3.11: Data coupling analysis. 
Table 3.2: Summary of instrument parameter selection.

\begin{tabular}{|c|c|c|c|c|}
\hline Parameter & LDA1 & LDA2 & PDA U1 & PDA U2 \\
\hline HV & 1000 & 1200 & 1000 & 1000 \\
\hline Signal gain & $24 d b$ & $24 d b$ & & \\
\hline Signal to noise ratio & $0 d b$ & $0 d b$ & & \\
\hline Level valadation & 2 & 2 & & \\
\hline Wavelenght $\left(\lambda_{b}\right)$ & & & $514.5 \mathrm{~nm}$ & $488.0 \mathrm{~nm}$ \\
\hline Transmitter focal length & & & $160.0 \mathrm{~mm}$ & $160.0 \mathrm{~mm}$ \\
\hline Receiver focal length (Photodetector) & & & $310.0 \mathrm{~mm}$ & $310.0 \mathrm{~mm}$ \\
\hline Beam Diameter & & & $1.35 \mathrm{~mm}$ & $1.35 \mathrm{~mm}$ \\
\hline Frequency shift & & & $40 \mathrm{M} \mathrm{Hz}$ & $40 \mathrm{M} \mathrm{Hz}$ \\
\hline Fring spacing & & & $38.0 z \mu m$ & $38.0 \mu \mathrm{m}$ \\
\hline Scattering angle & & & $30^{\circ}$ & $30^{\circ}$ \\
\hline Fring direction & & & Negative & Negative \\
\hline Scattering mode & & & Refraction & Refraction \\
\hline Aperture mask & & & Mask A & Mask A \\
\hline Spherical validation band & & & $20 \%$ & $20 \%$ \\
\hline Max detactable diamter & & & $40.43 \mu \mathrm{m}$ & $40.43 \mu \mathrm{m}$ \\
\hline Relative refraction index & & & 1.33 & 1.33 \\
\hline
\end{tabular}


combining both measurements using this technique.

\subsubsection{Parameter Selection Summary}

Table 3.2 shows a summary of all instrument parameters selected for this study.

\subsection{Spiriva Respimat Aerosol characterization using ACI (Andersen Cascade Impactor)}

\subsubsection{Experimental Apparatus}

Experiments are performed in both normal (40-50\% relative humidity $(\mathrm{RH})$ ) and humid ( $>90 \% \mathrm{RH}$ ) air to study the ACI aerosol deposition using commercially available Spiriva Respimat. Table 3.3 outlines the four test cases carried out in the current study.

Table 3.3: Controlled parameters used in the study.

\begin{tabular}{|c|c|c|}
\hline Test Case & Flow Rate $(L /$ min $)$ & Relative Humidity $(\%)$ \\
\hline 1 & 28.3 & $40-50$ \\
\hline 2 & 28.3 & $>90$ \\
\hline
\end{tabular}

Figure 3.12 shows a schematic of the experimental setup used in this study. As described in Table 3.3, for test cases 1 and 2, the inhaler is connected directly to the induction port (IP) simulating the upper airway of an 8-stage Andersen Cascade Impactor (ACI, stages 0 to 7) (MSP Corporation, Shoreview, MN), as illustrated in Figure 2.4.

As seen in Figure 3.12, the entire setup is placed in a sealed, temperature-andhumidity-controlled environment. The temperature is kept constant $\left(22 \pm 2^{\circ} \mathrm{C}\right)$ throughout the entire experiment and the humid environment is created using a Duracraft DCM200 2 Gallon Cool Mist Humidifier. A DHT22 temperature-humidity sensor 


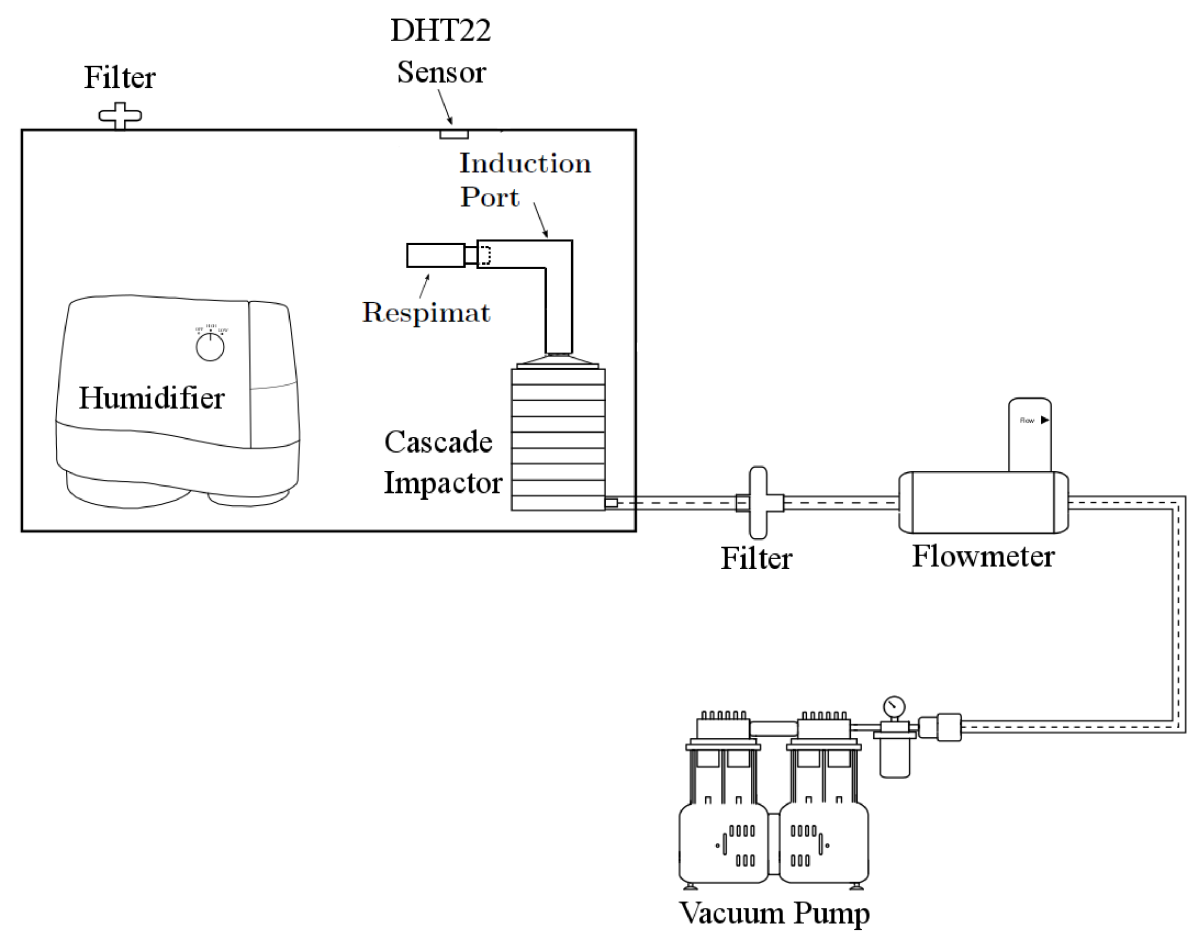

Figure 3.12: Schematic of the experimental setup used for Repimat.

(Adafruit Industries, LLC., New York, NY) connected to an Arduino UNO Rev3 (Arduino, LLC., Somerville, MA) is used to measure both the temperature and humidity of the environment. The DHT22 sensor is placed directly at the IP level of the ACI to measure the humidity of the air entering the system. To simulate the inspiratory flow rate of a healthy male subject, the method used by Alhegagi [57], and Ogrodnik et al.,[59] is utilized, through which the ACI is connected in series to a Vital Signs RespirGard II 303 bacterial/viral filter (Vital Signs, Inc., Englewood, CO), a Brooks Mass Flow Meter 5863S (Brooks Instrument, LLC., Hatfield, PA), and a Welch Dry Vacuum Pump 2585B (Welch-Ilmvac, Niles, IL). The filter is placed between the ACI and the flow meter to collect any unwanted particles leaving the ACI. The flow meter is connected to a National Instruments Data Acquisition USB-6009 device (National Instruments Corporation, Austin, TX) and the readings are recorded with LabVIEW software. The flow rate is monitored and maintained at $28.3 \mathrm{~L} / \mathrm{min}$. 


\subsubsection{Experimental Procedure}

Prior to each experiment, each device under test is washed with dish soap, rinsed with water, and allowed to air dry. The cascade impactor is assembled and the experiments are connected as described previously. The Spiriva Respimat inhaler is primed by releasing 5 puffs in open air for first time use, and loaded onto the respective device. For test case 2, the humidifier is turned on for 30 minutes to allow the relative humidity to reach a steady $98-99 \%$. The vacuum pump is then run at a flow rate of $28.3 \mathrm{~L} / \mathrm{min}$ for at least 15 minutes to allow the flow to settle before starting the experiment. Twenty actuations of the Respimat are used with a 30 second intervals between each actuation. The vacuum pump is left running for an additional 60 seconds to allow the medication to properly deposit on the plates of the Anderson Cascade Impactor (ACI).

The experimental setup is then disassembled and prepared for washing. The ACI deposition plates are placed into separate Petri dishes with $15 \mathrm{~mL}$ of distilled water and are shaken for 1 minute each. The IP is washed with $15 \mathrm{~mL}$ of distilled water. Each component is left in their respective solution for 2 hours to allow for a consistent dissolution of the medication.

\subsubsection{Absorbance Measurement}

The deposition of the tiotropium bromide monohydrate for each test case is then determined by measuring the absorbency of each wash solution using the Agilent 8453 UV-Visible Spectrophotometer (Agilent Technologies, Santa Clara, CA). A calibration curve is necessary to relate the measured absorbency to the concentration of tiotropium bromide monohydrate. To generate such curve, a stock solution of tiotropium bromide monohydrate and distilled water (using $25 \mathrm{~mL}$ ) is prepared from $13.9 \mathrm{mg}$ of pure tiotropium bromide monohydrate (Sigma Aldrich Canada, Oakville, Canada). The 
stock solution is then diluted to known concentrations and the absorbency of these standard solutions is obtained using the Agilent spectrophotometer. It is found that the tiotropium bromide monohydrate has an absorption wavelength of $237 \mathrm{~nm}$.

Prior to measuring the absorbency of the wash solutions, each cuvette is washed 3 times with distilled water and primed 3 times with $0.5 \mathrm{~mL}$ of the wash solution under test. At least 2 absorbency readings are taken for each sample of the wash solutions. The concentration of tiotropium bromide monohydrate could then be found using the calibration curve. The mass deposition and particle size distribution can therefore be calculated.

\subsection{CCD High Speed Camera}

To further study the spray, a high speed digital videography is captured using the A Nanosense MK 3 CCD high speed camera to obtain qualitative spray characteristics such as travel distance and spray duration. The camera features a 10,000 fps recording at $1280 \times 1024$ pixels with an internal memory of 2 GB. Using the support rig described in the previous section, the Respimat SMI is mounted about $10-15 \mathrm{~cm}$ in front of a black sheet of cardboard as background. Additionally, a light source is mounted a $30-40 \mathrm{~cm}$ above the spray region with the high-speed camera positioned about $2 m$ away, as shown in Figure 3.13. The video, which is recorded at $500 \mathrm{fps}$ for $2 s$, is then used to 1) study the spray distance and duration, as well as, 2) to compare the spray characteristics with a pMDI. 


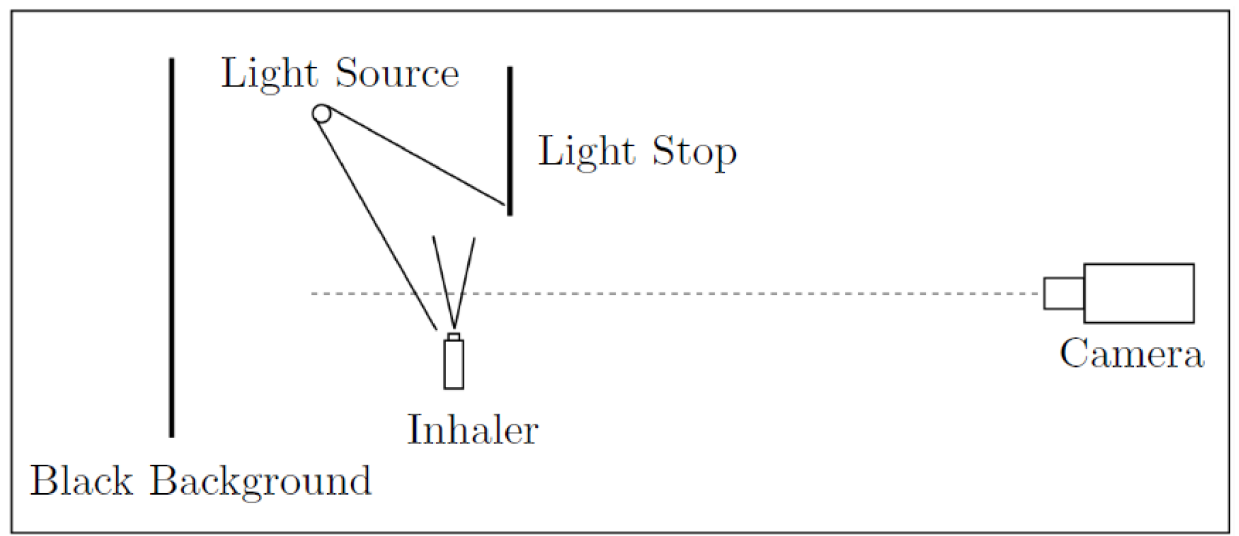

Figure 3.13: Schematic of high-speed camera setup. 


\section{Chapter 4}

\section{Numerical methods}

\subsection{Introduction}

Several previous studies have been implemented in a Computational Fluid Dynamics (CFD) software code to create a computational model for a pMDI spray. In this study, simulations are performed using ANSYS CFX. The puffer is connected to a simple geometry (called testbox) in order to generate a realistic approximation of the real-life environment of the system. This chapter presents an introduction for both the geometry that is used for the simulation and the theory that the software package, ANSYS CFX, uses to solve the unsteady Reynolds Averaged Navier-Stokes (URANS) equations.

\section{$4.2 \quad$ Geometry}

The testbox comprises of a simple cylindrical form with the dimensions of length $250 \mathrm{~mm}$ and diameter $400 \mathrm{~mm}$ representing a sample of a room environment as illustrated in Figure 4.1, where the puffer's mouthpiece is connected. The geometry of the Ventolin HFA inhaler is drawn using Creo Simulate software and then loaded into the ICEM CFD, a modeling and meshing software included in the ANSYS fluid flow simulation package. An unstructured mesh is created from the geometry and is created 
using tetrahedral elements as shown in Figure 4.2. In addition, a mesh intensity is placed inside the testbox and the puffer mouthpiece as shown in Figure 4.2. This is preformed on this region to capture details of the flow in this area.

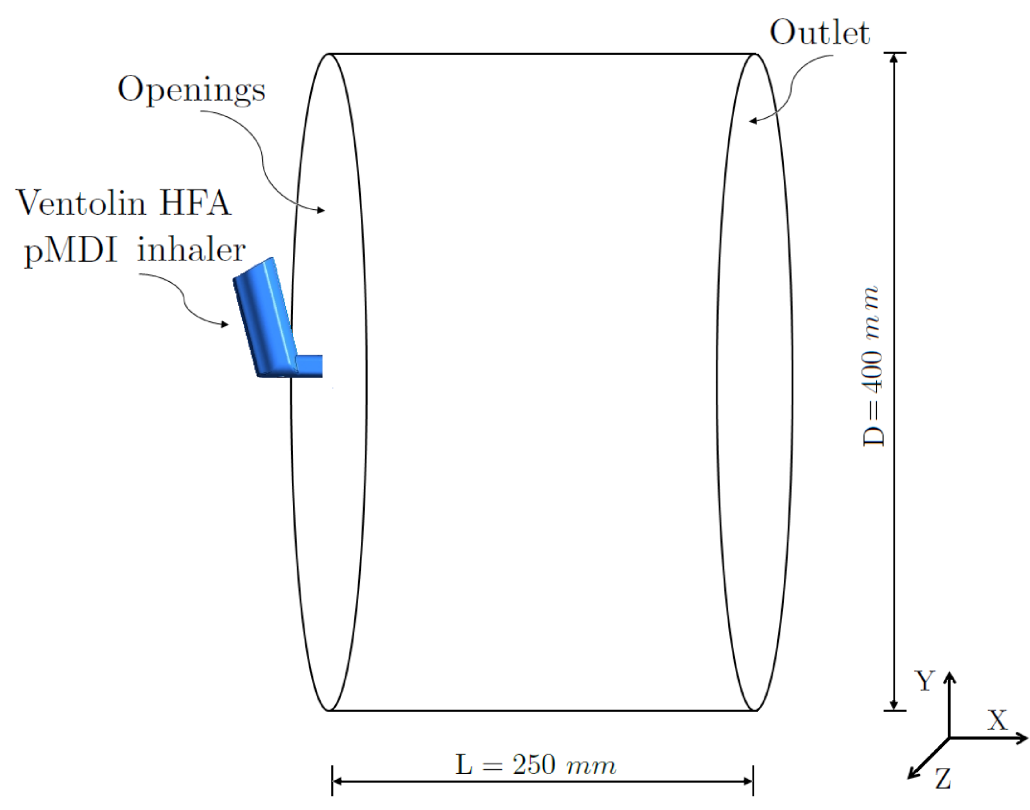

Figure 4.1: The testbox representation.

\subsection{Boundary conditions}

To present the nozzle inlet conditions and let the pMDI spray to deflect downward (spray axis to $-6^{\circ}$ and back to $0^{\circ}$ ) as described by Crosland [7]. The inlet is placed downstream of the actual nozzle, close to the actuator's cylindrical stem (on the surface of the expansion chamber at the nozzle location). Also, to obtain a more accurate mesh for the nozzle inlet, its diameter is increased to $1.49 \mathrm{~mm}$ from the actual nozzle diameter of $0.25 \mathrm{~mm}$. In order to demonstrate the deflection in the spray angle emitted from the actuator, Matida and Alhegagi [84] developed the following equation as a function of 


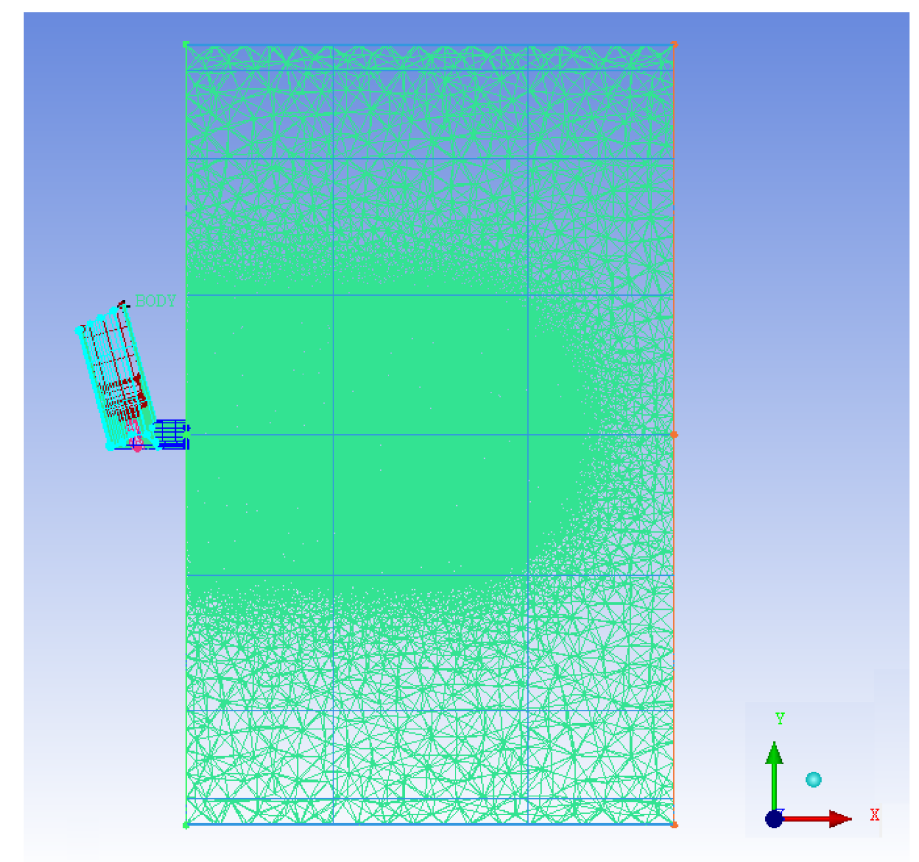

Figure 4.2: Geometry made in ICEM by ANSYS and the mesh intensity.

time, which could be stated as:

$$
\alpha=-\frac{1490.5}{t^{1.75}}\left[1-\exp \left(-0.000727 t^{2.50}\right)\right]
$$

The boundary conditions for the faces of the testbox are defined as openings, enabling the free motion of the air, as well as the spray particles. Only the outlet face is assumed as an outlet region with a zero-gradient static gauge pressure. Also, the opening space between the actuator and the canister at the top is assumed as an opening with a zero-gradient static gauge pressure region having a value of $0 \mathrm{~Pa}$. The pMDI actuator and canister boundaries are considered as 'No-slip wall', trapping all the particles that collide with them.

\subsection{Drug properties and spray characterisation}

The particle size data are measured using the PDA in the experimental stage, which is then used as inlet data for the simulation. The simulations are initialized by releasing 
10,000 particles into the compactional domain over the actuation time of each puffer from the nozzle inlet. Two way coupling is used to describe the particulate phase. Data is fitted to the probability distribution function known as the Rosin-Rammler distribution, as found in the experimental results, which will be presented later. The CFD solver accepts this distribution type by inserting its corresponding parameters, such as the Rosin-Rammler size, and power. Therefore, for each type of inhaler, its Rosin-Rammler size which is the mean diameter can be found in Table 5.3. The RosinRammler power $d$ can be calculated via the following equation [85]:

$$
R=\exp \left[-\left(\frac{d}{d_{e}}\right)^{k}\right]
$$

where $R$, the mass fraction, $d_{e}$, the mean diameter, and $k$, the spread parameter which also can be found in Table 5.4. In addition, the inlet velocity for the puffer is set to $80 \mathrm{~m} / \mathrm{s}$, as after the actuation the propellant will evaporate nearly immediately. This leaves only the medication to be inhaled, and travelling at around this velocity [7]. Table 4.1 summarizes all the parameters that are adopted in the simulation for this type of pMDI used in the study.

Table 4.1: Parameters used in the simulation.

\begin{tabular}{|c|c|c|c|}
\hline \multirow{2}{*}{ pMDI type } & \multicolumn{3}{|c|}{ parameter } \\
\cline { 2 - 4 } & $d_{e}(\mu m)$ & $k$ & $d(\mu m)$ \\
\hline Ventolin HFA & 3.750 & 1.315 & 8.637 \\
\hline
\end{tabular}

\subsection{Single phase (air) flow}

The simulations are carried out using ANSYS CFX. The governing equations for 
the mass and momentum conservation used are unsteady Reynolds-Averaged NavierStokes (URANS), in order to study the flow within the puffer and the testbox. For the momentum equations, the velocity at a point is considered as a sum of the mean and the fluctuating components as follows:

$$
u_{i}=\overline{U_{i}}+u_{i}^{\prime}
$$

The continuity equation, accounting for mass conservation can be express as:

$$
\frac{\partial \rho}{\partial t}+\frac{\partial \rho \overline{U_{i}}}{\partial x_{i}}=0
$$

where $\rho$, is the density of the fluid, and $t$, is time [86]. Therefore, the Reynolds averaged momentum equation can be written as:

$$
\frac{\partial\left(\rho \overline{U_{i}}\right)}{\partial t}+\frac{\partial\left(\rho \overline{U_{j} U_{i}}\right)}{\partial x_{j}}=-\frac{\partial \bar{P}}{\partial x_{j}}+\frac{\partial}{\partial x_{j}}\left(\mu\left(\frac{\partial \overline{U_{i}}}{\partial x_{j}}+\frac{\partial \overline{U_{j}}}{\partial x_{i}}\right)\right)+\rho g_{i}-\frac{\partial}{\partial x_{j}}\left(\rho \overline{u_{i}^{\prime} u_{j}^{\prime}}\right)
$$

Where $\mu$, is the dynamic viscosity, $\bar{P}$, is the time-averaged pressure, $g_{i}$, is the component of the gravitational acceleration in the direction of $x_{i}$, and the final term, $\left(\rho \overline{u_{i}^{\prime} u_{j}^{\prime}}\right)$ is known as the Reynolds stresses. The Shear Stress Transport (SST) turbulence model is a two-equation model that uses an extended Boussinesq relationship to relate the Reynolds stress terms in the URANS equations to the velocity gradient in the flow, which can then be given as [86];

$$
\left.-\rho \overline{u_{i}^{\prime} u_{j}^{\prime}}=\mu_{t}\left(\frac{\partial \overline{U_{i}}}{\partial x_{j}}+\frac{\partial \overline{U_{j}}}{\partial x_{i}}\right)\right)-\frac{2}{3} \rho k \delta_{i j}
$$

Where $\delta_{i j}$, is the Kronecker delta, given as; 


$$
\delta_{i j}= \begin{cases}1 & \text { when } i=j \\ 0 & \text { whwn } i \neq j\end{cases}
$$

Also, the term $k$, represents the turbulent kinetic energy per unit mass which is expressed as;

$$
k=\frac{1}{2}\left(\overline{u_{i}^{2}}\right)
$$

Where $i=1,2,3$. And the turbulent viscosity term $\mu_{t}$, is calculated from the following equation as;

$$
\mu_{t},=C_{\mu} \rho \frac{k^{2}}{\varepsilon}
$$

Where $C_{\mu}$, is a model constant, and $\varepsilon$, is the dissipation and calculated as $\varepsilon=\nu \overline{\frac{\partial u_{i}}{\partial x_{i}} \frac{\partial u_{j}}{\partial x_{j}}}$

The development SST model was as a result of the inability of both existing $\mathrm{k}-\varepsilon$ and $\mathrm{k}-\omega$ models to fully describe the behavior of the flow [87]. The $\mathrm{k}-\varepsilon$ model can capture turbulence accurately in a turbulent region, whereas the $\mathrm{k}-\omega$ turbulence model works best near the wall, inside the boundary layer. Therefore, the SST turbulence model is a hybrid turbulence solver which employs the $\mathrm{k}-\varepsilon$ and the $\mathrm{k}-\omega$ model. This is achieved by replacing the rate of viscous dissipation for the product of the turbulent frequency and the turbulent kinetic energy [86]. Thus, the governing transport equation for the turbulent kinetic energy per unit mass, $k$, in tensor form can be stated as:

$$
\frac{\partial(\rho k)}{\partial t}+\frac{\partial\left(\overline{U_{i}} \rho k\right)}{\partial x_{i}}=\frac{\partial}{\partial x_{i}}\left(\left(\mu+\frac{\mu_{t}}{\sigma_{k 3}}\right) \frac{\partial k}{\partial x_{i}}\right)+P_{k}-\beta^{\prime} \rho k \varpi
$$

where $P_{k}$, is the shear production of turbulence, $\varpi$, is the turbulence frequency, and $\sigma_{k 3}$, and are $\beta^{\prime}$ constants. The transport equation for the turbulent frequency, $\varpi$, in tensor form is then: 
$\frac{\partial(\rho \varpi)}{\partial t}+\frac{\partial\left(\overline{U_{i}} \rho \varpi\right)}{\partial x_{i}}=\frac{\partial}{\partial x_{i}}\left(\left(\mu+\frac{\mu_{t}}{\sigma_{\varpi 3}}\right) \frac{\partial \varpi}{\partial x_{i}}\right)+\left(1-F_{1}\right) 2 \rho \frac{1}{\sigma_{k 3} \varpi} \frac{\partial k}{\partial x_{i}} \frac{\partial \varpi}{\partial x_{i}}+\alpha_{3} \frac{\varpi}{k} P_{k}-\beta_{3} \rho \varpi^{2}$

where $\sigma_{\varpi 3}, \alpha_{3}$, and $\beta_{3}$ are all constants fitted to the SST model, and $F_{1}$, is a blending factor that is dependent on the region of flow [86]. The transport equations for turbulent kinetic energy and turbulent frequency both follow a similar form. The first term on the left-hand side of equation (4.10) and (4.11) is composed of a time rate of change of the transported variable, which is followed by a term defining the transport of the variable by convection. The right-hand side of the transport equations are composed of the transport of the variable by turbulent dissipation, the rate of production of the variable and the rate of dissipation of the variable [86].

In addition, Equations 4.10 and 4.11 are known as Baseline model. The turbulent viscosity in this case is defined as;

$$
\nu_{t}=\frac{\mu_{t}}{\rho}
$$

This model improves the representation of the flow, but it over predict the results of shear stress. However, the SST model is used to compensate this, where the turbulent viscosity is calculated in the following way, to better model the eddy-viscosity.

$$
\nu_{t}=\frac{a_{a} k}{\max \left(a_{1} \omega, S F_{2}\right)}
$$

The SST model is also introducing a blending function $F$ and $F_{2}$ to obtain a smooth transition between the $\mathrm{k}-\omega$ model near the wall and the $\mathrm{k}-\varepsilon$ far from it where $F_{1}$ is zero.

$$
F_{1}=\tanh \left(\arg _{1}^{4}\right)
$$


Where;

$$
\begin{gathered}
\arg _{1}=\min \left(\max \left(\frac{\sqrt{k}}{\beta^{*} \omega y}, \frac{500 \nu}{y^{2} \omega}\right) \frac{4 \rho k}{C D_{k \omega} \sigma_{\omega 2} y^{2}}\right) \\
C D_{k \omega}=\max \left(2 \rho \frac{1}{\sigma_{\omega 2} \omega} \nabla k \nabla \omega, 1.0 \times 10^{-10}\right)
\end{gathered}
$$

And for the blending function $F_{2}$ used for SST modelling;

$$
F_{2}=\tanh \left(\arg _{2}^{2}\right)
$$

Where;

$$
\arg _{2}=\max \left(\frac{2 \sqrt{k}}{\beta^{*} \omega y}, \frac{500 \nu}{y^{2} \omega}\right)
$$

where $y$ is the distance to the nearest wall and the model coefficients are shown in Table 4.2 .

Table 4.2: Coefficients of the SST model.

\begin{tabular}{|c|c|c|c|c|c|}
\hline$\sigma_{k 1}$ & $\sigma_{\omega 1}$ & $\beta_{1}$ & $a_{1}$ & $\beta^{*}$ & $\kappa$ \\
\hline 0.85 & 0.50 & 0.0750 & 0.31 & 0.09 & 0.41 \\
\hline
\end{tabular}

\subsection{Lagrangian particle tracking (particulate phase)}

Multiphase flow is modelled to provide an accurate simulation of the behaviour of salbutamol sulphate in the inhaler and its testbox. To initialize the simulations, Lagrangian particles (300,000 particles) are released into the domain over $180 \mathrm{~ms}$ (the actuation time of the Ventolin HFA puffer) from the nozzle inlet at $80 \mathrm{~m} / \mathrm{s}$. Two way 
coupling is used to describe the particulate phase, and the turbulence intensity of the kinetic energy at the inlet is kept at the software default by $5 \%$. Salbutamol sulphate is specified as a particle transport fluid with a Schiller Naumann drag model, used to model the drag around the particles. The Schiller Naumann drag model is ideal for small particle Reynolds numbers when fluid particles behave similar to solid particles and for flow around a single sphere [88].

That at viscous regime near the wall which for low particle Reynolds numbers, the drag coefficient for flow past spherical particles is computed analytically. The drag coefficient for a sphere in Stokes flow

$$
C_{D}=24 / R e \quad R e \ll 1
$$

For particles that have large Reynolds numbers, which are adequately large for inertial effects to dominate viscous effects (Newton's regime), the drag coefficient is given by the following equation that becomes independent of Reynolds number:

$$
C_{D}=0.44, \quad 1000 \leq R e \leq 1-2 \times 10^{5}
$$

In the transitional region between Stoke drag and Newtonian drag, where predicting analytical solution for a falling sphere is consequently complicated. Therefore, to overcome this issue an empirical expression is applied to calculate drag in this regime, which Schiller Naumann drag model.

$$
\begin{gathered}
F=C_{D} \rho_{f} \frac{U^{2}}{2} A \\
C_{D}=\frac{24}{R e}\left(1+0.15 R e^{0.687}\right)
\end{gathered}
$$

ANSYS CFX adjusts this to ensure the correct limiting behavior in the inertial 
regime by taking:

$$
C_{D}=\max \left(\frac{24}{R e}\left(1+0.15 R e^{0.687}\right), 0.44\right)
$$

ANSYS CFX has the functionality to perform Lagrangian particle tracking, ideal for particles with small diameters in multiphase flow [89,90]. The three dimensional Lagrangian equation states that;

$$
\frac{d u_{p, i}}{d t}=\frac{\left(u_{f, i}-u_{p, i}\right)}{\tau_{p}}-\mathrm{g}_{i}\left(1-\frac{\rho_{f}}{\rho_{p}}\right)
$$

Where $u_{p, i}$ is the particle velocity components $(\mathrm{m} / \mathrm{s}), u_{f, i}$ is the instantaneous fluid flow components at the particle location $\mathrm{m} / \mathrm{s}$, and $\mathrm{g}_{i}$ is the gravitational components $\mathrm{m} / \mathrm{s}^{2}$. The relaxation time of the particle can be expressed as;

$$
\tau_{p}=\frac{\rho_{p} d_{p}^{2}}{18 \mu \alpha}
$$

Where;

$$
\alpha=1+0.15 R e_{p}^{0.687}
$$

$R e_{p}$ is the particle Reynolds number.

When RANS equations are used, each particle has an instantaneous velocity divided into two components, mean and fluctuating;

$$
u_{f, i}=\bar{u}_{f, i}+u_{f, i}^{\prime}
$$

Turbulent tracking, also known as the random walk model, considers both the mean and fluctuating components of the instantaneous velocity. The inclusion of the fluctuating component results in particle dispersion in turbulent flow since particles can follow 
independent tracks at each injection point.

To model this turbulent particle dispersion, each particle is assumed to interact with eddies as it travels throughout the domain. Each eddy has a characteristic length, lifetime and velocity. When a particle interacts with an eddy, the fluctuating component of the instantaneous fluid velocity is assumed to be equal to the fluctuating velocity of the eddy. This causes the fluctuating velocity $u_{f, i}^{\prime}$ to dominate. A particle ends its interaction with an eddy if the lifetime of the eddy is exceeded or the particle travels the eddy length. At this point, the particle is assumed to enter another new eddy.

\subsection{Grid Convergence studies}

The main objective of the grid convergence study is to guarantee that the URANS simulations are spatially converged in such a way that they reliably captured the interactions of the flow inside the puffer and the testbox, though minimizing the required computational time for the simulation by removing the unnecessary mesh. Also, error and uncertainty can rise in CFD simulations, from both, the grid refinement and the geometry simplification which is also known as the physical error. The study is carried out by generating the geometry in ICEM CFD, then the number of elements is sufficient to be used in the simulation. This number should be appropriate and large enough to obtain results with a suitably sufficient amount of accuracy. The strategy also, allowed a reduction in the computational time that would otherwise be necessary for a complete simulation.

A spatial convergence analysis is performed using three different geometries with different dimensions in the testbox size as listed in Table 4.3 below.

Different grid sizes between 2,400,000 and 19,100,000 elements are used for the convergence, in respect of verifying that a grid size that will maintain the solution within one order of magnitude of the finest possible mesh. Therefore, the grid convergence is carried-out in a steady-state simulation, where an outlet velocity of $80 \mathrm{~m} / \mathrm{s}$ is calculated 
Table 4.3: Testboxes dimension..

\begin{tabular}{|c|c|c|}
\hline Geometry No. & Diameter $\mathrm{mm}$. & Length $\mathrm{mm}$. \\
\hline Geometry 1 G1 & 200 & 200 \\
\hline Geometry 2 G2 & 300 & 250 \\
\hline Geometry 3 G3 & 400 & 250 \\
\hline
\end{tabular}

at the nozzle, at a downwards angle of 6 for the Ventolin HFA puffer. To define the optimal mesh size, a characteristic of the simulation is designated and studied over the selected different mesh sizes. A grid is counted to be converged after the selected characteristic values converge, consequently, any addition in the mesh elements would give similar results, whereas the smaller sized mesh can be used without losing accuracy in results [91-93]. Therefore, to achieve this convergence study, the centre line velocity can be chosen as the criteria as in Hayase et al. [91]. The velocity along the centre line in the $\mathrm{x}-\mathrm{y}$ plane of the geometry is used to analyse the convergence, as illustrated in Figure 4.3. Consequently, by using this criteria it is clear how many elements are needed to generate accurate simulation results, by comparing the each successive set of results against each other for each grid size considered.

In terms of temporal analysis studies, an important parameter in CFD simulations is to know the time steps that need to accurately represent the flow within the tested domain, specifically in transient simulations to guarantee that the full behaviour of the flow is captured. Therefore, at the beginning of each iteration in the simulation, the time step should be appropriately small in order to ensure the stability of the numerical solution procedure. The time step limitations of a numerical solution procedure are typically stated in the dimensionless method of a Courant number which represents how the flow is moving through the mesh. The local Courant number is calculated in 


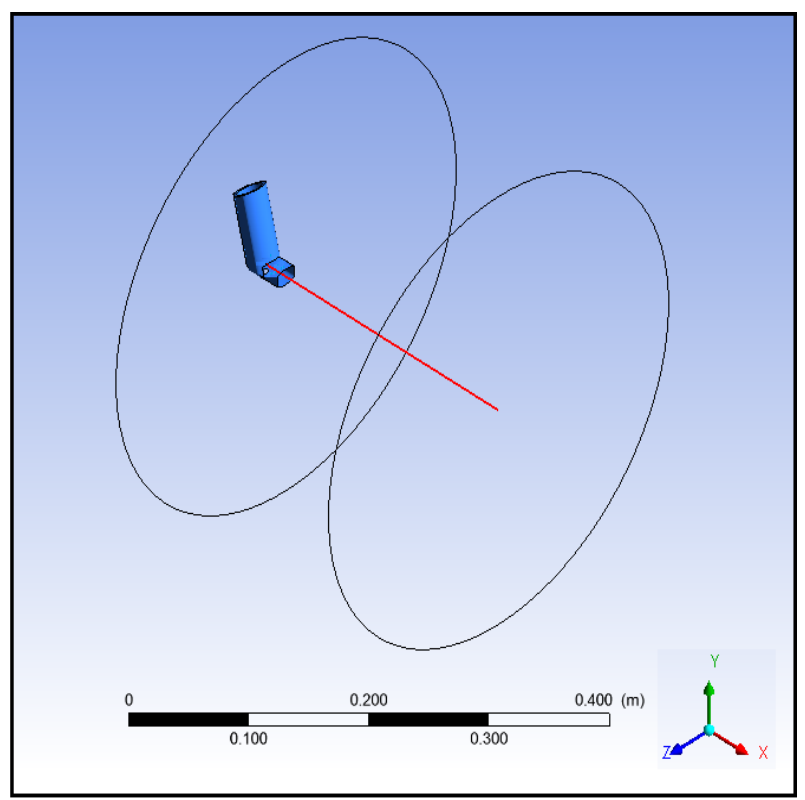

Figure 4.3: Areas of interest for grid convergence studies.

the following way:

$$
\text { Courant number }=\frac{U \triangle t}{\triangle x}
$$

Where $U$ the flow velocity, $\Delta t$ the time step size, and $\Delta x$ the element size in the direction of flow. Its interpretation that if the Courant number is greater than unity, means that the fluid flow transfers out of multiple cells in the domain at each time step, eventually resulting in a divergent solution. Otherwise, if the Courant number is equal or less than unity, this means that the fluid flow moves from one cell to the next within one time step. Time steps of $0.1 \mathrm{~ms}, 0.01 \mathrm{~ms}$, and $0.001 \mathrm{~ms}$ are used in the temporal convergence analysis, and time step cannot be increased without compromising the stability of the solution process. However, it is found from the analysis that using a small time step of $\Delta t=0.01 \mathrm{~ms}$ resulting in a maximum Courant number of CFL $=0.89$.

\subsubsection{Grid and Domain Convergence Studies Results}

A grid convergence study is conducted to guarantee that the results are accurate and independent of the mesh resolution. As discussed above, the domain consists of 
tetrahedral elements ranging from 2.4 million to 19.1 million tetrahedral elements. In addition, a domain convergence study was carried out using three sets of geometries labelled G1, G2 and G3 where the number of elements is increased in each geometry for studying a mesh convergence study in a steady state simulation.

Figure 4.4 shows the velocity distribution along the centerline as a function of the distance from the orifice for various domains and mesh sizes. As can be seen in Figure 4.4(a), domain G1 with 8.7 million elements produces close results to meshes with 12.3 million elements. Whereas, meshes with 2.5 million and 2.7 million elements produced much different results and would not be in a suitable size range for this simulation. Similarly, this was the case with increasing the element numbers in G2 as can be seen inFigure 4.4(b). In the third geometry G3 (Figure 4.4(c)), a mesh with 12.4 million elements would produce the best results but this mesh would require extensive computational time and power to accomplish. However, the results of this analysis are as follows.

From the spatial analysis, a comparison is made between the three geometries where G1 is composed of 8.7 million elements, G2 has 8.2 million elements and G3 has 7 million elements as illustrated in Figure 4.4(d). The G3 mesh with 7 million elements yields better results than the G1 and G2 having greater number of elements. Also, this mesh would demand a smaller amount of time to converge, and thus the third geometry with 7 million elements is considered sufficient for this analysis.

From the temporal analysis, within the tested time steps it is found that a time step of $0.01 \mathrm{~ms}$ is adequate and gains sufficient details from the flow. Whereas, for the $0.1 \mathrm{~ms}$ time steps is not fully capturing the results and $0.001 \mathrm{~ms}$ time steps require an inefficient amount of time to complete simulation. As mentioned earlier, to monitor the accuracy and stability, in a transient simulation study, careful application of Courant number is necessary. Therefore, the selected time steps of $0.01 \mathrm{~ms}$ averaged the Courant number consistently, allowing it to remain below one. In addition, from the analysis 


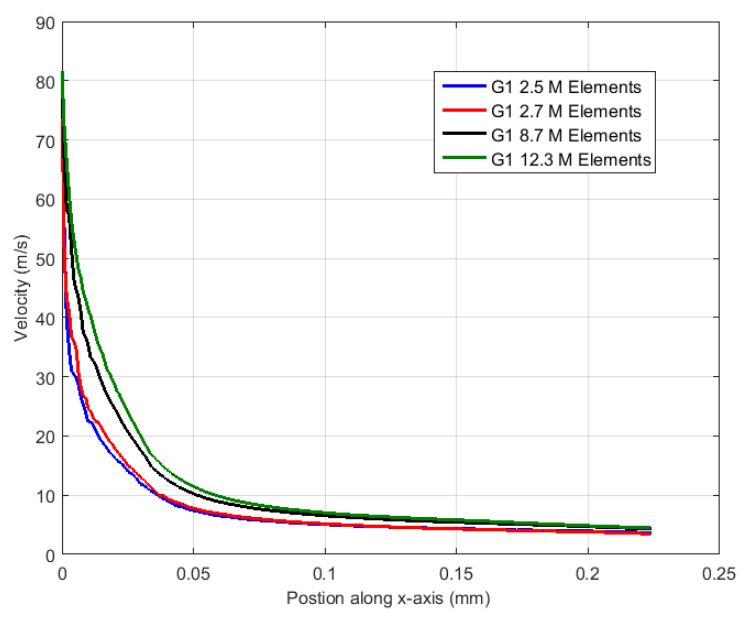

(a) Velocity along x-axis for G1.

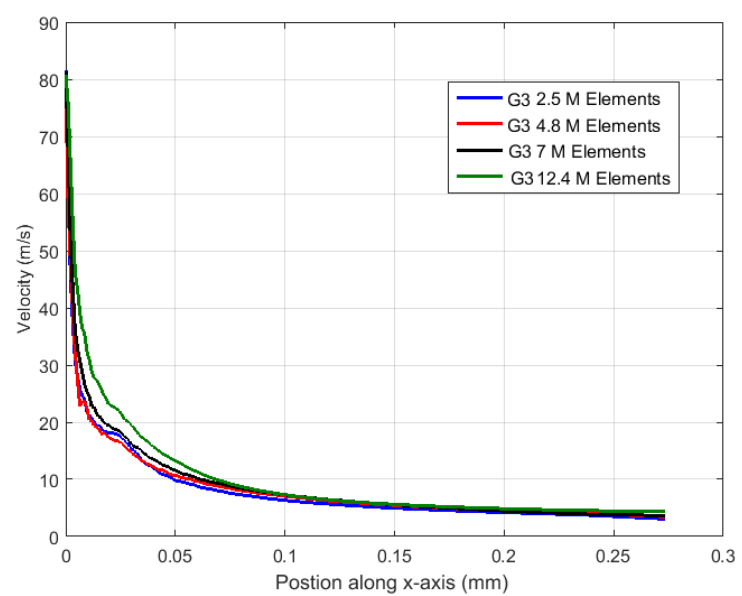

(c) Velocity along x-axis for G3.

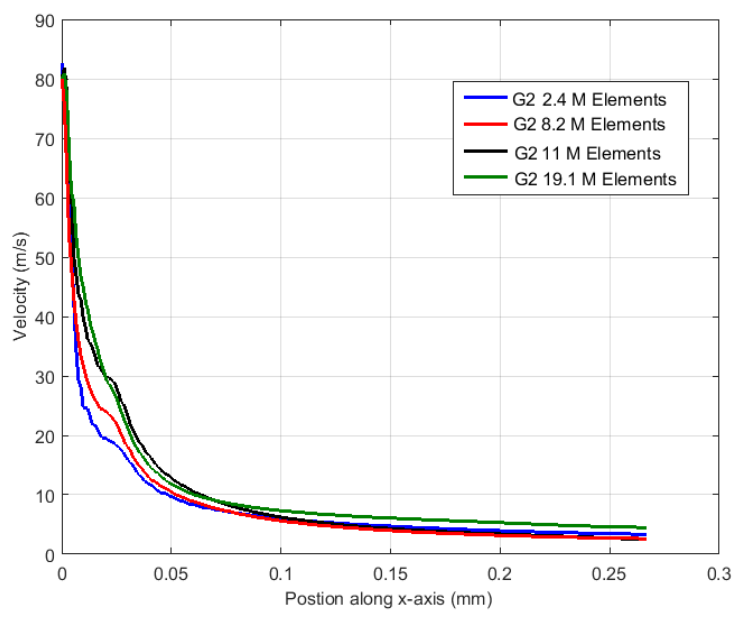

(b) Velocity along $\mathrm{x}$-axis for G2.

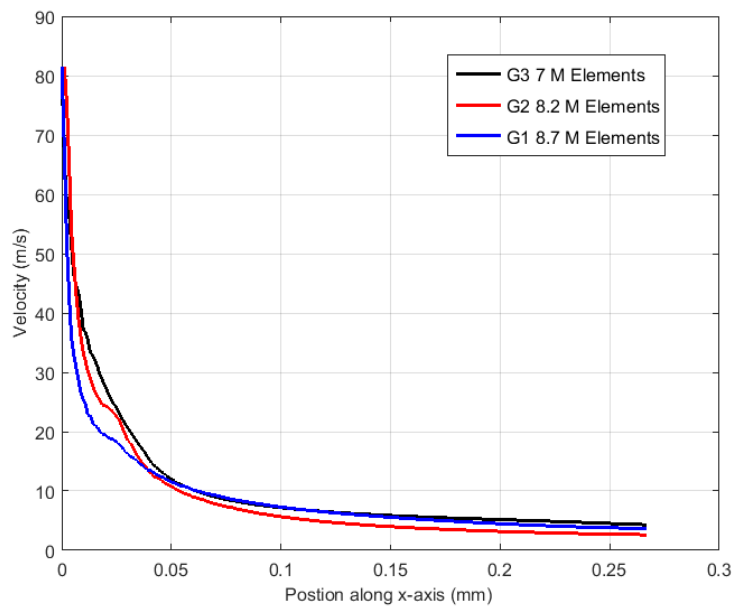

(d) Comparison of the velocity for G1, G2, and G3.

Figure 4.4: Velocity along the $\mathrm{x}$-axis for tested geometries. 
the required amount of time that would allow particles to fully travel through testbox after actuation is found to be $320 \mathrm{~ms}$. 


\section{Chapter 5}

\section{Results and Discussions}

\subsection{Introduction}

In this chapter the experimental results of the tested inhalers, along with the numerical study, are both presented in terms of a temporal and spatial analysis. Firstly, the experimental results of the PDA, ACI and are presented, which are then followed by the uncertainty analysis of the results and High Speed Camera results. The chapter closes by presenting the numerical study for the transient results of the velocity of the pMDI spray. Also, the numerical study shows how the CFD software code can predict the pMDI spray behaviour.

\subsection{Experimental results}

\subsubsection{Temporal analysis of inhalers spray}

The spray issued from a pMDI for each tested inhaler was characterised temporally at each of the axial stations as illustrated in Figure 5.1 (Figure 3.5 is repeated here

for clarification), in terms of the particle velocity and size. It was found by Liu et al. [17] and Dunbar [12] that particles close to the center of a spray plume have 
consistently higher velocities. Therefore, the mean velocity was used to characterize the inhaler spray velocity. As mentioned earlier, that the distance from the nozzle orifice to the mouthpiece edge of the pMDI is $25 \mathrm{~mm}$, whereas this distance is $6.5 \mathrm{~mm}$ for the Spiriva Respimat. A single point measurement is taken at this location (-18.5, 0) for the Spiriva Respimat only. To compare all tested inhalers at the same locations the distance of $25 \mathrm{~mm}$ from the nozzle orifice is set as the first of three axial stations selected for measurement as $x=0 \mathrm{~mm}$, which is the edge of the mouthpiece for the pMDI, where information of the atomization process could be obtained (Dunbar [12]). The second location at $x=75 \mathrm{~mm}$, which is generally considered as the estimation of the distance from the mouthpiece edge to the oropharynx, where the highest impaction occurs [12] and the third location at $x=100 \mathrm{~mm}$, which represents the stage after the oropharynx. The zero time in all figures means the time when the particles start crossing the measurement volume.

\subsubsection{Velocity analysis of the inhaler spray}

To study the temporal evolution of the spray velocity, measurements at the three locations from the origin, on the centerline, are taken to evaluate an ensemble-meann velocity. At the first station (at $x=0 \mathrm{~mm}$ ) the transient event duration for the four inhalers is different for each, where it is approximately $180 \mathrm{~ms}$ for the Ventolin HFA. Whereas, the transient event duration for Novo-Salbutamol HFA, Airomir HFA, and Apo-Salvent CFC Free is approximately $290 \mathrm{~ms}, 220 \mathrm{~ms}$, and $220 \mathrm{~ms}$, respectively. However, the transient event duration for Spiriva Respimat inhaler is approximately $1500 \mathrm{~ms}$.

Figure 5.2(a) shows the raw data for the particle velocities of five actuations for the Ventolin HFA. The red solid line represents the moving average velocity of the released particles using Equation 2.3 for each 50 particles, while the dashed black line illustrates the mean velocity using Equation 2.1. The mean velocity is $43.20 \mathrm{~m} / \mathrm{s}$ and there was 

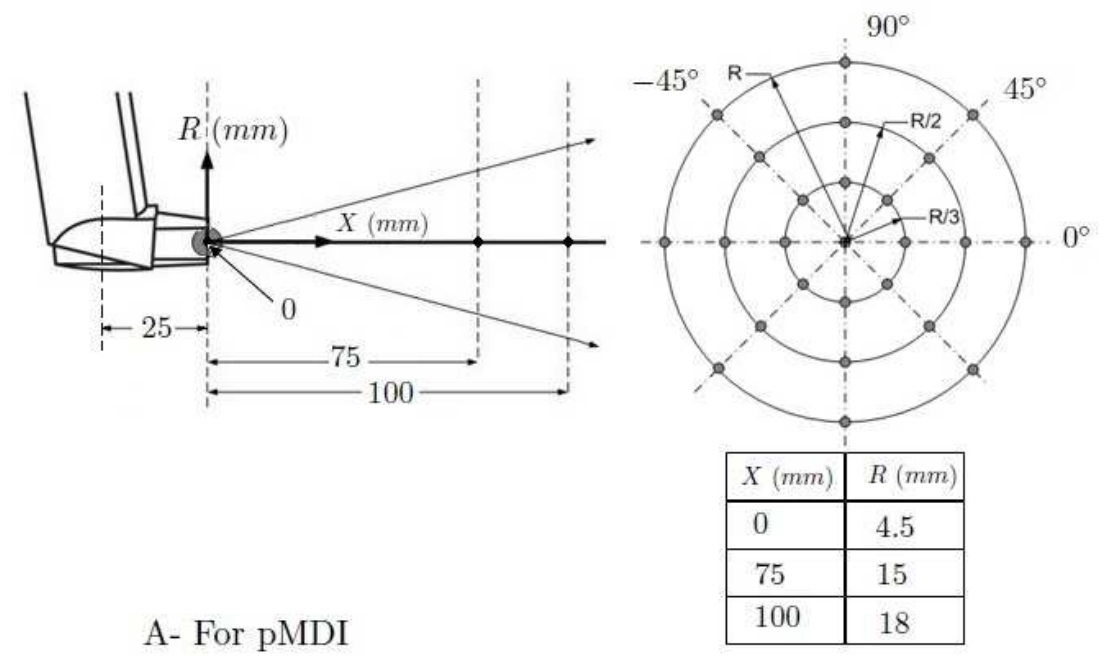

A- For pMDI
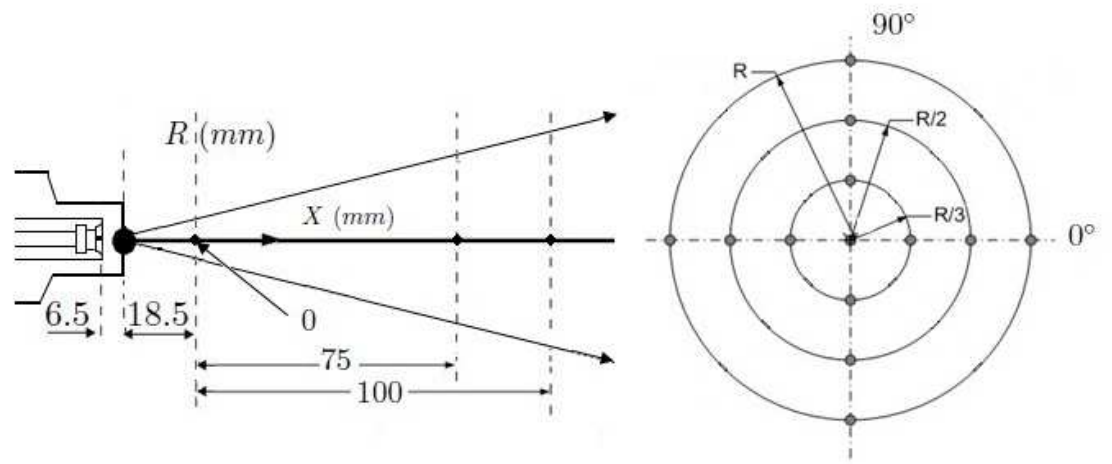

B- For Spiriva Respimat

\begin{tabular}{|l|l|}
\hline$X(\mathrm{~mm})$ & $R(\mathrm{~mm})$ \\
\hline 0 & 4.5 \\
\hline 75 & 6 \\
\hline 100 & 8 \\
\hline
\end{tabular}

Figure 5.1: Experimental measurement locations. 
a high spray density with a moving average velocity of $38.77 \mathrm{~m} / \mathrm{s}$ occurring at the beginning of the actuation. Then, the moving average velocity increased to $52.85 \mathrm{~m} / \mathrm{s}$, which is the first peak at $2 \mathrm{~ms}$, followed by a sharp drop until a time of $20 \mathrm{~ms}$. The second peak appears at $60 \mathrm{~ms}$ with a moving average velocity of $48.50 \mathrm{~m} / \mathrm{s}$. Then, the moving average velocity decreased with fluctuation until $70 \mathrm{~ms}$, where it went below the mean velocity and dropped slightly downwards. The figure also shows a drop in velocity between the two peaks.

Figure 5.2(b) illustrates the raw data for the particle velocities of Novo-Salbutamol HFA for five actuations to demonstrate the repeated transient event. Here the dark solid line illustrates the moving average velocity for the emitted particles, where the dashed line shows the mean velocity, which is $26.94 \mathrm{~m} / \mathrm{s}$. At the beginning of the actuation the high spray density produced a moving average velocity of $28.00 \mathrm{~m} / \mathrm{s}$. The first peak appears at $2 \mathrm{~ms}$ with a moving average velocity of $33.80 \mathrm{~m} / \mathrm{s}$. Then the moving average velocity declined quickly and reached $18.00 \mathrm{~m} / \mathrm{s}$ at a time of $20 \mathrm{~ms}$. Subsequently, the moving average velocity raised slightly with fluctuations until the $70 \mathrm{~ms}$ mark was reached where it went above the mean velocity, until the second peak at $120 \mathrm{~ms}$, with a moving average velocity of $33.70 \mathrm{~m} / \mathrm{s}$. Finally, the moving average velocity dropped moderately until the end of the spray enent.

Figure 5.2(c) shows the raw data for the particle velocities of Airomir HFA for five actuations where the mean velocity in this inhaler is $32.40 \mathrm{~m} / \mathrm{s}$. At the start of the actuation, the high spray density produced a moving average velocity of $26.50 \mathrm{~m} / \mathrm{s}$. The first peak is at $6 \mathrm{~ms}$ with a moving average velocity of $42.00 \mathrm{~m} / \mathrm{s}$. Then the moving average velocity declined quickly and reached $27.89 \mathrm{~m} / \mathrm{s}$ at a time of $40 \mathrm{~ms}$. With fluctuations, the moving average velocity increases slightly until a second peak is reached at $100 \mathrm{~ms}$ with a moving average velocity of $46.16 \mathrm{~m} / \mathrm{s}$. Finally, the moving average velocity dropped moderately until the end of the spray event.

Raw data for the Apo-Salvent CFC Free inhaler is shown in Figure 5.2(d), where 


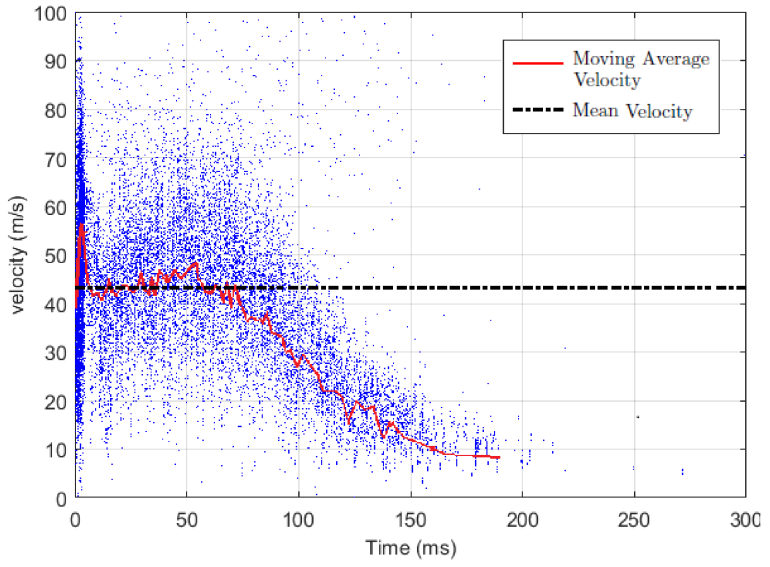

(a) For the Ventolin HFA inhaler.

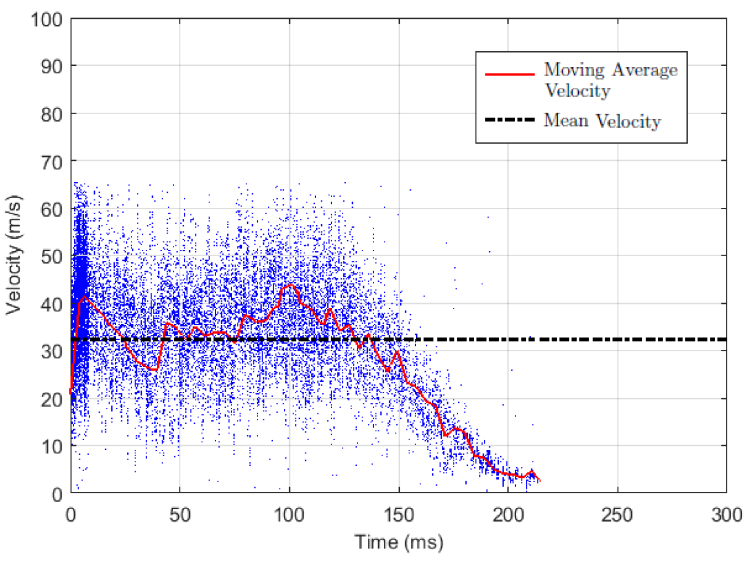

(c) For the Airomir HFA inhaler.

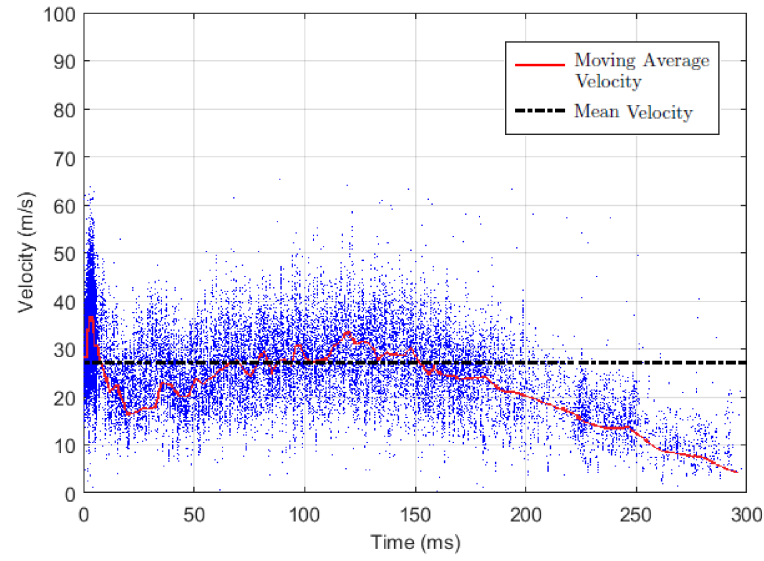

(b) For the Novo-Salbutamol HFA inhaler.

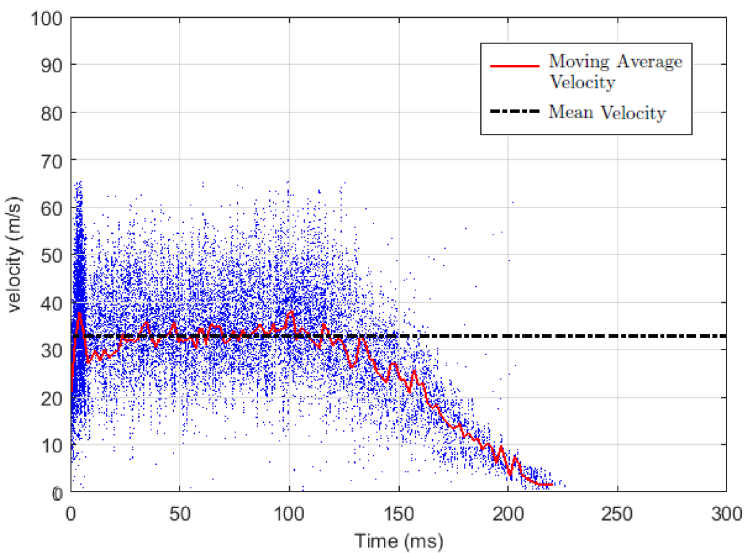

(d) For the Apo-Salvent CFC Free inhaler.

Figure 5.2: Raw data for the particle velocities for five actuations for each inhaler at $x=0 \mathrm{~mm}$. 
it displays particle velocities for five actuations. In this case the mean velocity is $32.87 \mathrm{~m} / \mathrm{s}$. In the high spray density region at the mouthpiece of the inhaler the particles reach a moving average velocity of $23.00 \mathrm{~m} / \mathrm{s}$. A first peak appears at $5 \mathrm{~ms}$ with a moving average velocity of $41.50 \mathrm{~m} / \mathrm{s}$. Then, the peak declines sharply until it drops below the mean velocity, before it increases slightly with fluctuations around the mean velocity until a second peak appears at $100 \mathrm{~ms}$. Thereafter, it decreases with fluctuations until the spray event is completed.

Secondly, at the $75 \mathrm{~mm}$ and $100 \mathrm{~mm}$ stations, only Ventolin HFA inhaler produced two peaks in velocity at the $75 \mathrm{~mm}$ station. At $100 \mathrm{~mm}$ location however, a first peak appears and then the velocity fluctuates around the mean velocity, producing a very small peak, before it declines until the end of the spray. For the other remaining inhalers the velocity behaves in a similar manner as all have a high density region at the start of the spray with no peak, then the velocity fluctuates around the mean until the end of the spray event. Figures 5.3 and 5.4 demonstrate these cases for both locations.

Figure 5.5 shows that for the time-dependent mean spray velocities of the four different pMDI brands, the velocity has two peaks at two different times at $(0,0)$. As can be seen in Figure 5.5(a) where the velocity of Ventolin HFA is compared against the results obtained by Crosland et al. [14] and Dunbar [12]. Two velocity maxima exist for the same location at $(0,0)$; the first as the spray is leaving the mouthpiece, $2 \mathrm{~ms}$ after actuation, and the second appears $60 \mathrm{~ms}$ later. This pattern is present with decreasing radial distance at $(0,-1.5)$, but with a reduction in spray velocity. The two velocity peaks at this position is delayed; the first occurring at $4 \mathrm{~ms}$ and the second at $65 \mathrm{~ms}$. This pattern also appears at location $(0,1.5)$ with less velocity compared to location $(0,0)$ but higher than $(0,-1.5)$. There is only one peak as the spray leaves the mouthpiece for both location $(0,-2.25)$ and $(0,2.25)$. This result indicates a deflection in spray velocity; a phenomenon also observed for the other three inhalers at $(0,0)$.

Figure 5.5(b) illustrates that the first peak occurs for Novo-Salbutamol HFA at 


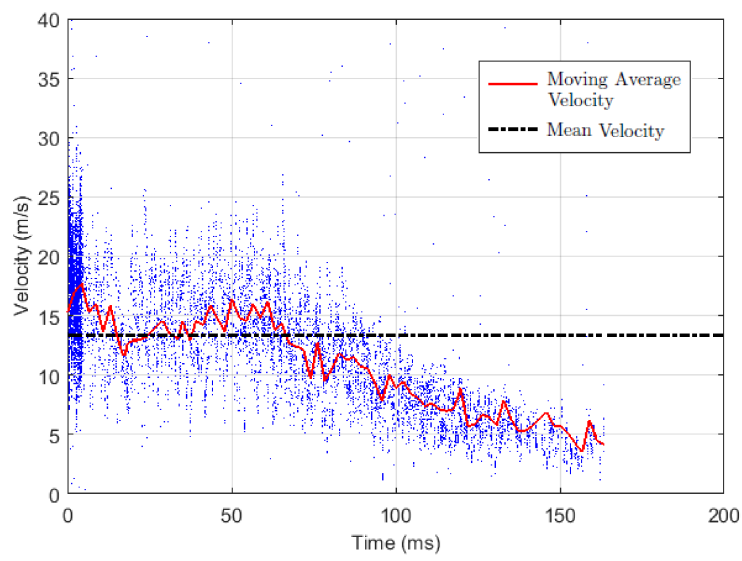

(a) For the Ventolin HFA inhaler.

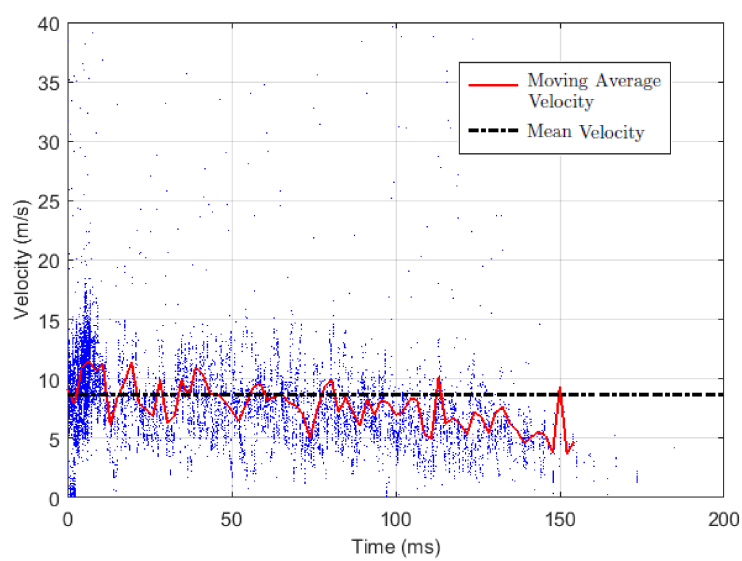

(c) For the Airomir HFA inhaler.

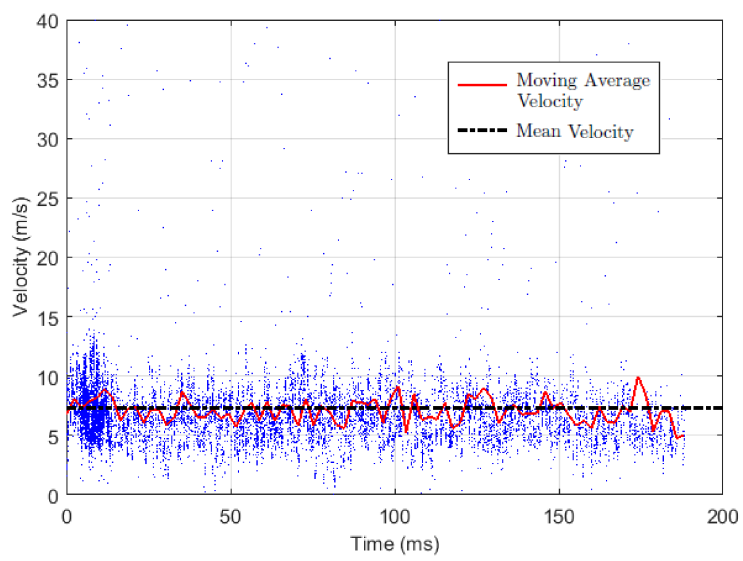

(b) For the Novo-Salbutamol HFA inhaler.

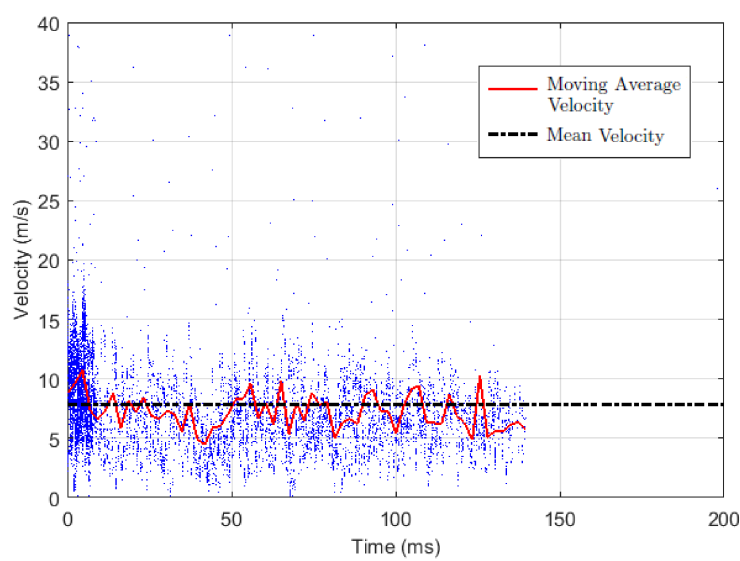

(d) For the Apo-Salvent CFC Free inhaler.

Figure 5.3: Raw data for the particle velocities for five actuations for each inhaler at $x=75 \mathrm{~mm}$. 


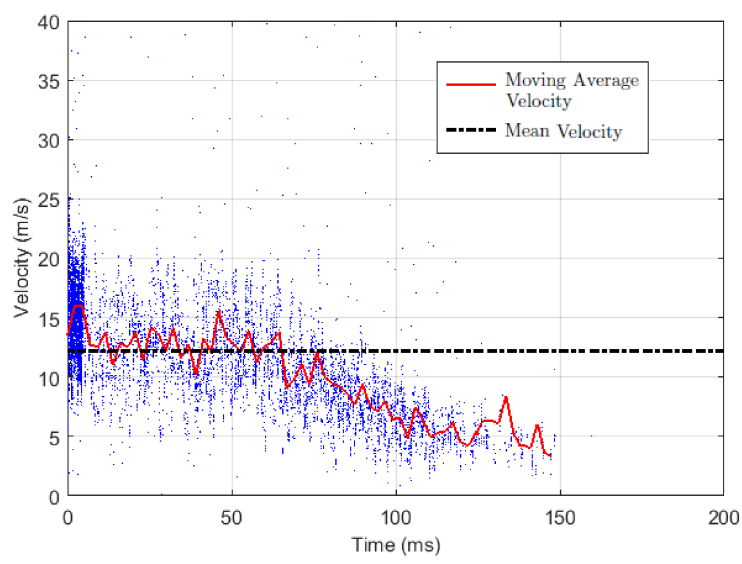

(a) For the Ventolin HFA inhaler.

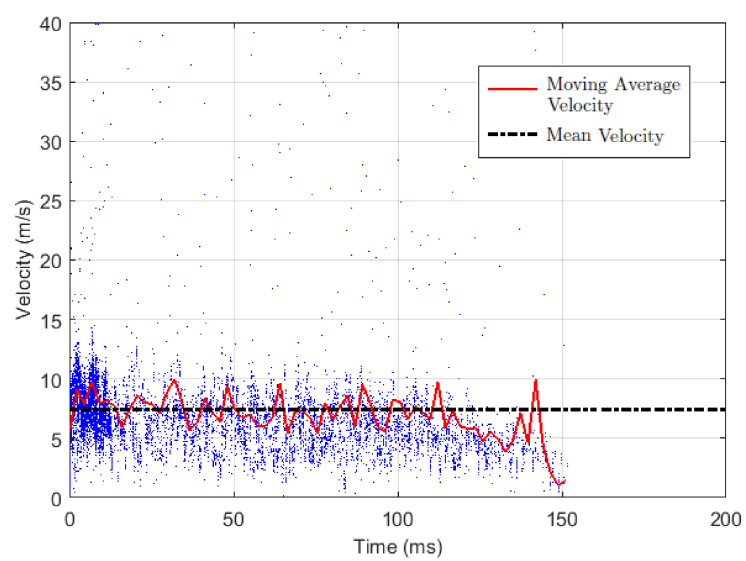

(c) For the Airomir HFA inhaler.

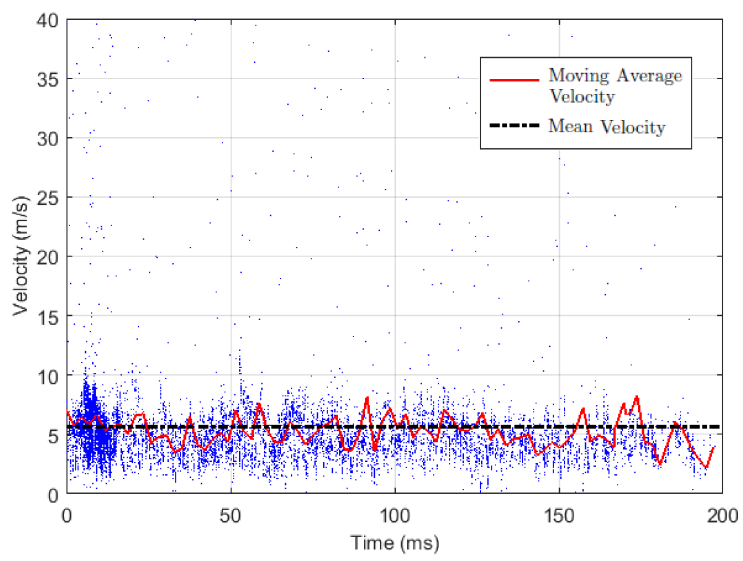

(b) For the Novo-Salbutamol HFA inhaler.

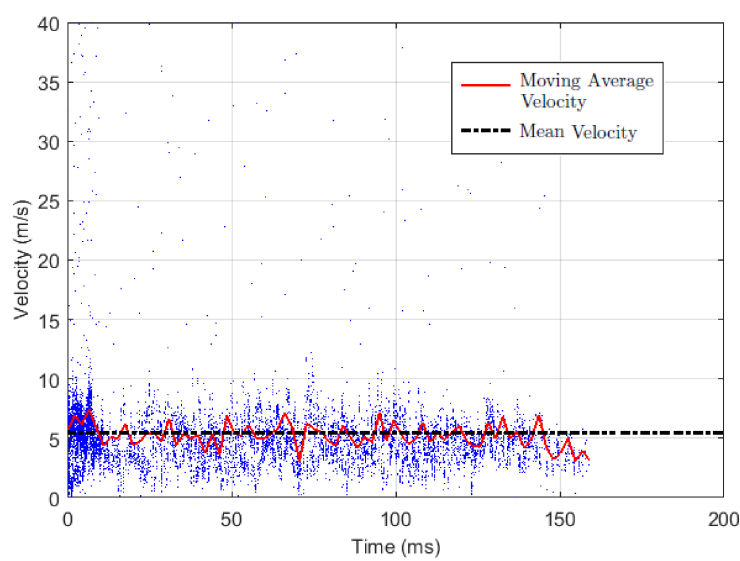

(d) For the Apo-Salvent CFC Free inhaler.

Figure 5.4: Raw data for the particle velocities for five actuations for each inhaler at $x=100 \mathrm{~mm}$. 
$2 \mathrm{~ms}$, the same as Ventolin HFA, whereas it occurs at $5 \mathrm{~ms}$ for the other two. The second velocity peak occurs 95 ms later for both the Airomir HFA 5.5(c), and the ApoSalvent CFC Free 5.5(d). Novo-Salbutamol HFA shows a greater delay for the second peak as it appears $115 \mathrm{~ms}$ after the first one occurs (again, Ventolin HFA exhibits higher velocity in comparison to the rest of tested inhalers).

For Novo-Salbutamol HFA Figure 5.5(b), both locations $(0,1.5),(0,-1.5)$, and $(0,-2.25)$ have two peaks, their first peaks occor at 2, 5, and $7 \mathrm{~ms}$ with velocities of $23,22.5$, and $17 \mathrm{~m} / \mathrm{s}$, respectively. Then, the velocity drops and increases slightly to approximately 20,22 , and $13.3 \mathrm{~m} / \mathrm{s}$. For location $(0,2.25)$ the first peak appears exactly at the time when the spray leaves the mouthpiece at a velocity of $21.5 \mathrm{~m} / \mathrm{s}$ then decreases until a time of $30 \mathrm{~ms}$ at a velocity of $15.5 \mathrm{~m} / \mathrm{s}$. Then, increases to $20.5 \mathrm{~m} / \mathrm{s}$ at $105 \mathrm{~ms}$ after which it declines to the end of the spray duration.

Regarding Airomir HFA measurement locations, Figure 5.5(c), shows that both (0, -1.5) and $(0,-2.25)$ demonstrate the same episode with two velocity maxima appearing at location $(0,0)$. The first occurs as the spray leaves the mouthpiece of the actuator which is at 6 and $7 \mathrm{~ms}$ with velocities of 32 and $23 \mathrm{~m} / \mathrm{s}$ respectively and the second at 110 and $155 \mathrm{~ms}$. At the upper locations $(0,1.5)$ and $(0,2.25)$ the spray velocity rises sharply as it leaves the mouthpiece at 4 and $2 \mathrm{~ms}$. Then, there is a slightly rise in their velocity, until a maxima appears at 63 and $84 \mathrm{~ms}$. The velocity declines until the end of the spray event.

Results for the remaining locations for Apo-Salvent CFC Free are in Figure 5.5(d). Only points $(0,-1.5)$ and $(0,-2.25)$ demonstrate the same trend as point $(0,0)$ with two peaks and a short time delay. The first peak appears at times 6 and $7 \mathrm{~ms}$ with velocity 33 and $29.5 \mathrm{~m} / \mathrm{s}$, respectively. Then the velocity drops for both and increases slightly to approximately 33.5 and $24.5 \mathrm{~m} / \mathrm{s}$ at times 114 and $117 \mathrm{~ms}$ then the reduces until the end of the spray. Locations $(0,1.5)$ has only one peak at a time of $39 \mathrm{~ms}$ with a velocity of $29 \mathrm{~m} / \mathrm{s}$ then, its drops until the end of the spray event. At location $(0,2.25)$ 


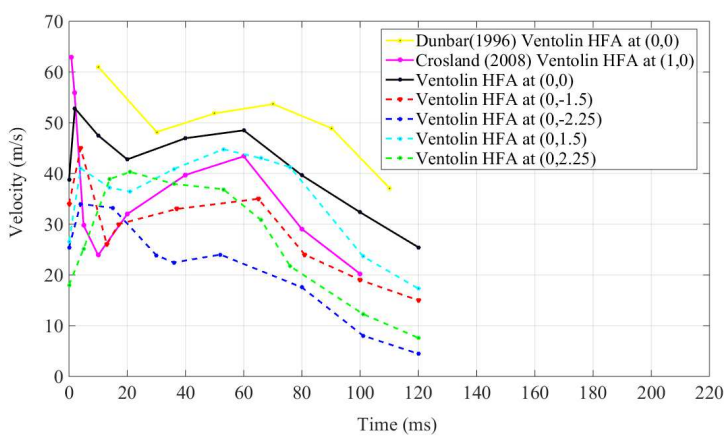

(a) For the Ventolin HFA inhaler.

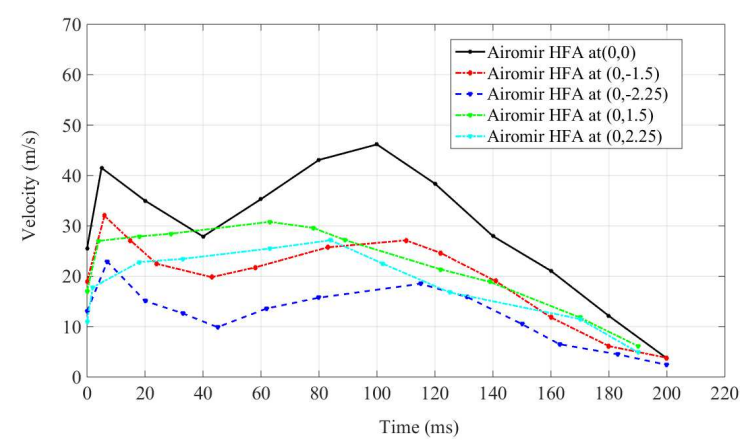

(c) For the Airomir HFA inhaler.

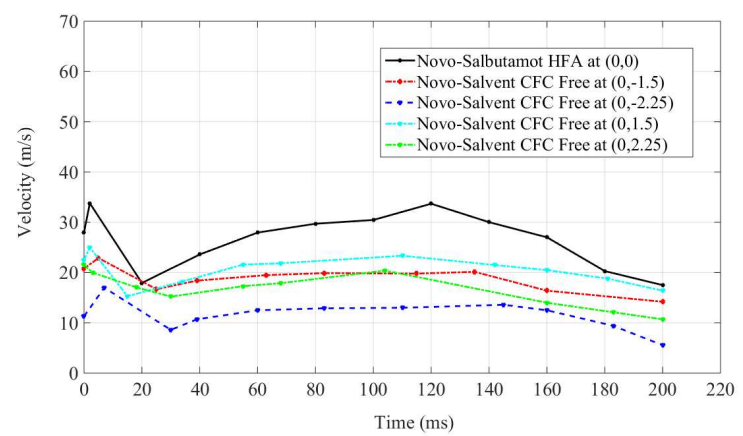

(b) For the Novo-Salbutamol HFA inhaler.

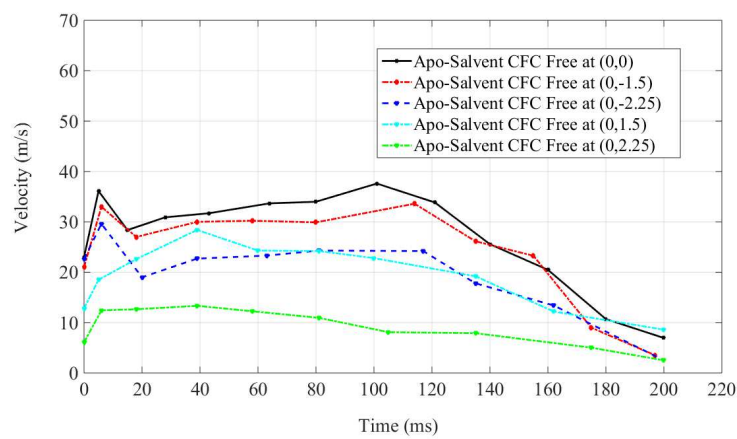

(d) For the Apo-Salvent CFC Free inhaler.

Figure 5.5: Temporal evolution of the measured spray velocity for each inhaler at $x=0$ $m m$.

the velocity increases sharply from $6 \mathrm{~m} / \mathrm{s}$ upon leaving the mouthpiece, to $12.5 \mathrm{~m} / \mathrm{s}$ at a time of $6 \mathrm{~ms}$. It then remains stable at approximately $13 \mathrm{~m} / \mathrm{s}$ until the $58 \mathrm{~ms}$ mark, after which the velocity drops until the end of the spray event.

This bimodal phenomenon was also observed by Dunbar [12] and [11] (both cited studies used the same technique as has been used in the present research), as well as Crosland et al. [14], who used a PIV technique. It is important to notice that Dunbar's [12] numerical study did not match with the experimental data, as the results did not show a bimodal trend in his simulation. The author hypothesized that there was an experimental error between the two maxima due to measurement dropout in dense spray 
regions. Consequently, Wigley et al. [11] concluded that an imbalance in mass-flow, which occurs within the expansion chamber between, in-flow and out-flow during the spray. It is likely that the measurement by Crosland et al. [14] showing that the spray deflecting during actuation (moving downwards and then upwards from the horizontal line) is the main reason for the presence of this bimodal behavior, appearing here.

For the temporal analysis velocity of the Spiriva Respimat inhaler, that the phenomenon of two peaks is not found for all stations as shown in Figure 5.1. For the location -18.5 $\mathrm{mm}$, which is at the inhaler mouthpiece is show the highest velocity compared to other locations. The moving average velocity starts with $17.5 \mathrm{~m} / \mathrm{s}$ at the beginning of the actuation, then decreases slightly but with a value above the mean velocity which is $10.95 \mathrm{~m} / \mathrm{s}$. Then at $120 \mathrm{~ms}$ it goes below the mean and keeps fluctuating around the mean until $1300 \mathrm{~ms}$ when it dropped below the mean which corresponds to the event when the particles have a large size as illustrated in Figure 5.6. All other locations have the same event that the velocity is fluctuating around the moving average velocity.

\subsubsection{Velocity contour}

Spray characteristics are measured at the $0 \mathrm{~mm}$ location for all tested pMDI inhalers. For each inhaler, a polar grid is optimized at this station to provide the spray with a cross sectional area. Figure 5.1 illustrates the measurement grid that has 25 downstream measurement locations. The data-set is then imported into MATLAB, where a custom script file derives a contour plot and a 3D surface of the cross-section using a cubic interpolation. Figure 5.7 demonstrates a contour plot of the axial velocity for the Ventolin HFA at different times. The spray characteristics demonstrate a slight asymmetrical behavior. The velocity profile changes considerably with the axial position. Figure 5.7(a) shows the velocity contour plot at a time of $0 \mathrm{~ms}$, that high velocity is observed on the radial distance $R / 3$ and the region on the lower $-45^{\circ}$ axis 


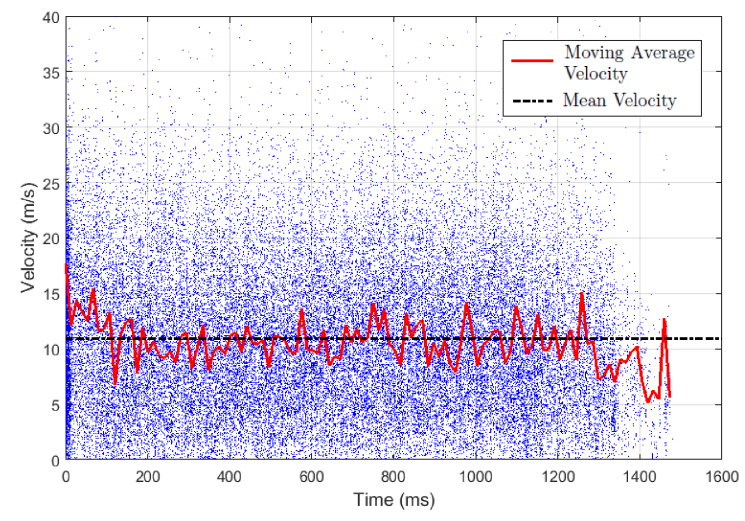

(a) At $x=-18.5 \mathrm{~mm}$.

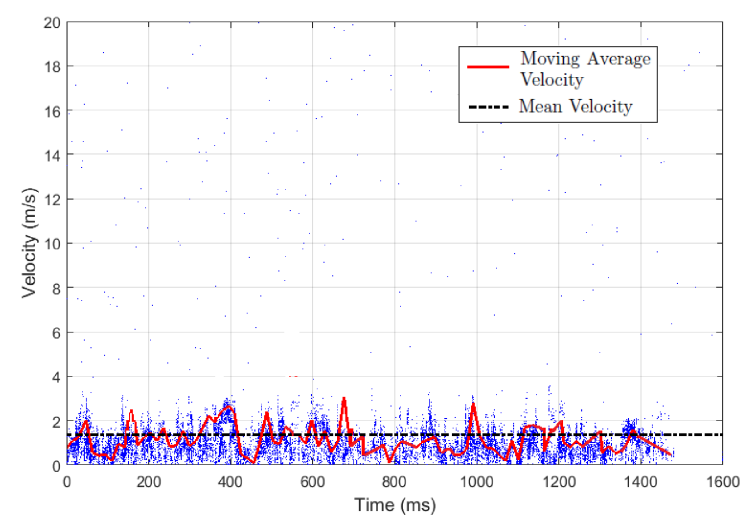

(c) At $x=75 \mathrm{~mm}$.

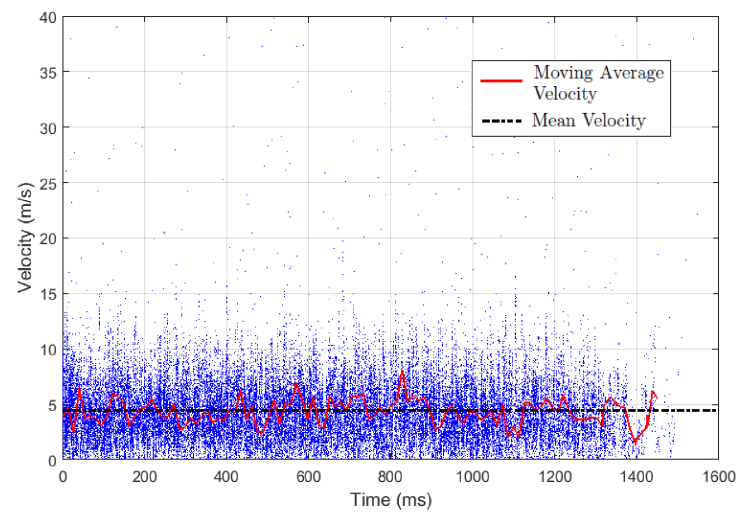

(b) At $x=0 \mathrm{~mm}$.

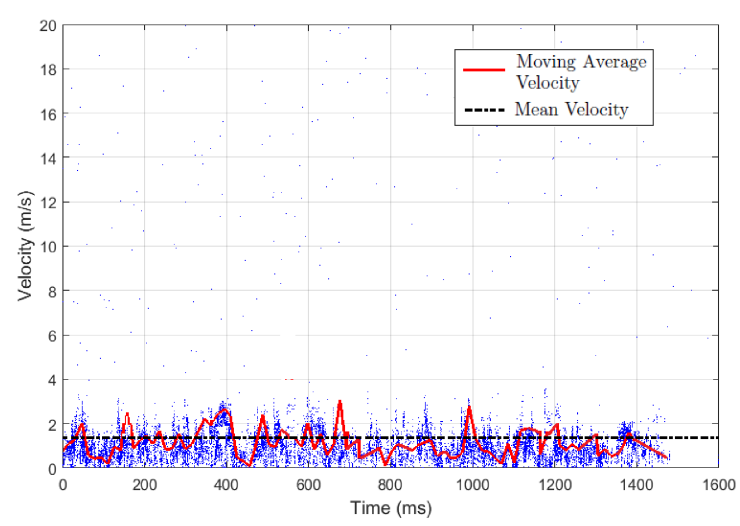

(d) At $x=100 \mathrm{~mm}$.

Figure 5.6: Raw data for the particle velocities for five actuations for Spiriva Respimat inhaler. 
(fourth quadrant) has the highest velocity. As the time increases to $2 \mathrm{~ms}$ the first peak is observed with the highest velocity at the center and lower region on the $90^{\circ}$ axis. Then, the velocity start to decline at $t=20 \mathrm{~ms}$ and rises to its second peak at the $60 \mathrm{~ms}$ mark, as in Figure 5.7(d). The velocity afterwards keeps decreasing until the end of the spray event Figures 5.7(e) and (f).

Figure 5.8 shows a contour plot of the axial velocity for the Novo-Salbutamol HFA inhaler at different interval times. The two peaks phenomenon can also be shown throughout these interval times in Figures5.8(b) and (e). In addition to the velocity increase at the actuation time of $0 \mathrm{~ms}$ (Figure 5.8(a)), it is decreases after the first peak at $20 \mathrm{~ms}$ where it then continue to rise to the second peak (Figure 5.8(d)) at $80 \mathrm{~ms}$.

For the other two pMDI inhalers Airomir HFA and Apo-Salvent CFC-Free both exhibit the same two peaks behavior. Their peaks occur at $5 \mathrm{~ms}$ and $100 \mathrm{~ms}$ with a drop in velocity after each peak. Airomir HFA inhaler has high velocity radial distance $R / 3$ and the region on the lower $90^{\circ}$ axis at its first peak and in upper $45^{\circ}$ axis (first quadrant) at its second peak. Whereas, it was in radial distance $R / 3$ in the region on the lower $45^{\circ}$ (third quadrant), $90^{\circ}$, and $-45^{\circ}$ (fourth quadrant) axes at its first peak, and only on the lower $90^{\circ}$ axis at its second peak. Figures 5.9 and 5.10 show these contour event for the mentioned inhalers.

\subsubsection{Particle diameter analysis of the inhaler spray}

Particle or droplet size could be classified as the most significant factor that impacts the deposition of aerosols in the lungs [60]. Figure 5.11(a) illustrates the raw data for the particle diameters over five actuations for Ventolin HFA. The red solid line represents the moving average diameter of the released particles using Equation 2.3 for each 50 particles, and the dashed black line illustrates the mean $D_{10}$, which is about $3.75 \mu \mathrm{m}$. At

the start of the actuation, when the spray density is high for about $4 \mathrm{~ms}$, the moving average diameter increases gradually from less than the mean $D_{10}$ to reach $5 \mu \mathrm{m}$ at 


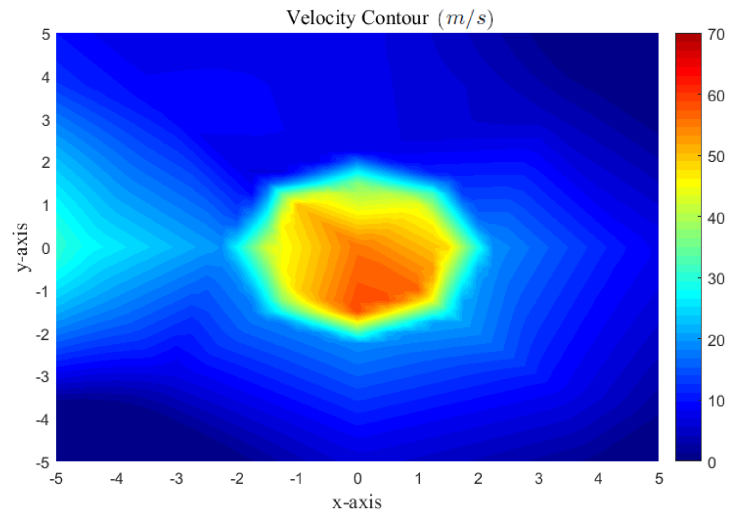

(a) Velocity contour plot at $\mathrm{t}=0 \mathrm{~ms}$.

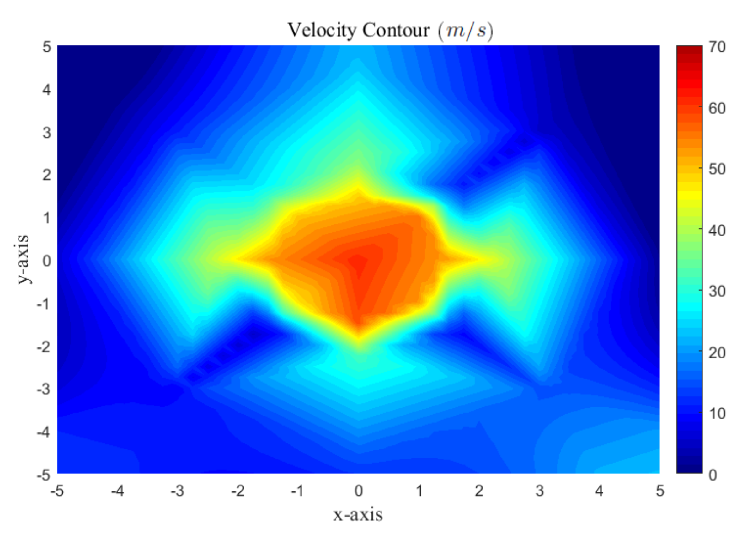

(c) Velocity contour plot at $\mathrm{t}=20 \mathrm{~ms}$.

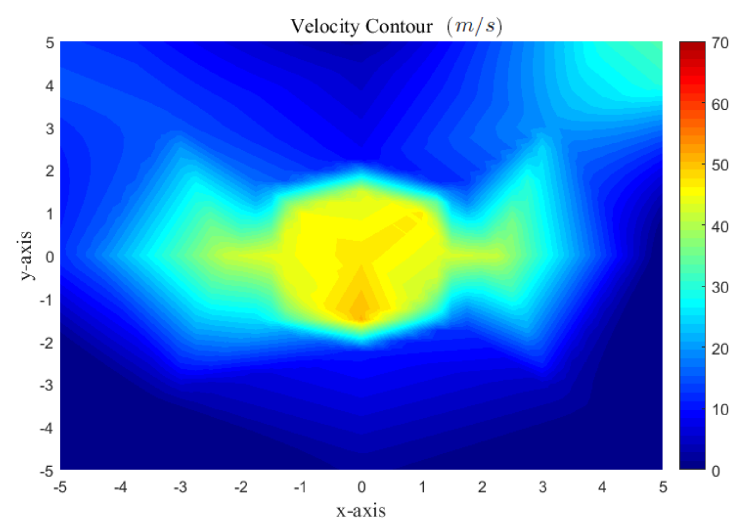

(e) Velocity contour plot at $\mathrm{t}=100 \mathrm{~ms}$.

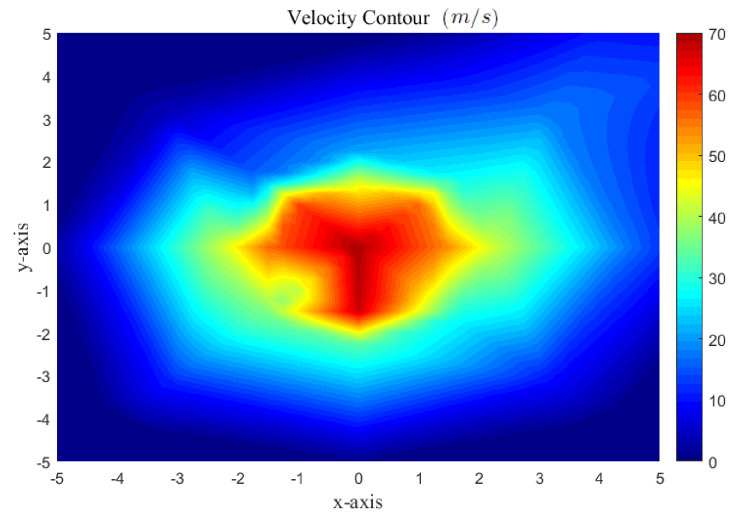

(b) Velocity contour plot at $\mathrm{t}=2 \mathrm{~ms}$.

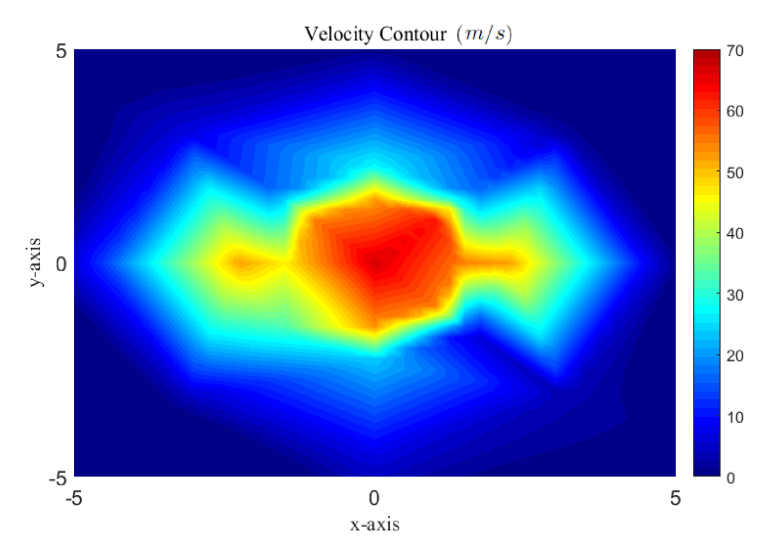

(d) Velocity contour plot at $\mathrm{t}=60 \mathrm{~ms}$.

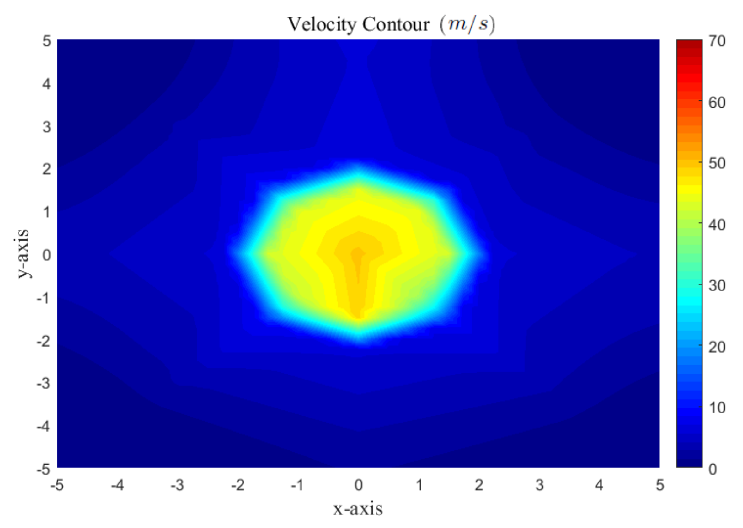

(f) Velocity contour plot at $\mathrm{t}=180 \mathrm{~ms}$.

Figure 5.7: Ventolin HFA inhaler velocity contour plot from PDA measurement at $x=0$ $m m$. 


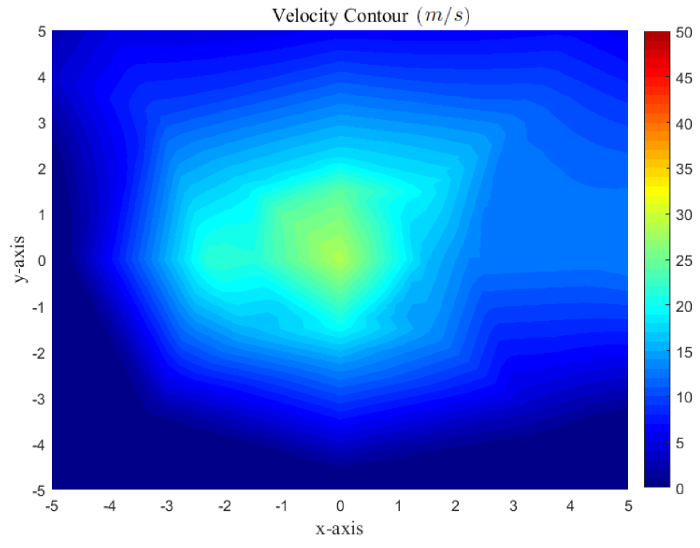

(a) Velocity contour plot at $\mathrm{t}=0 \mathrm{~ms}$.

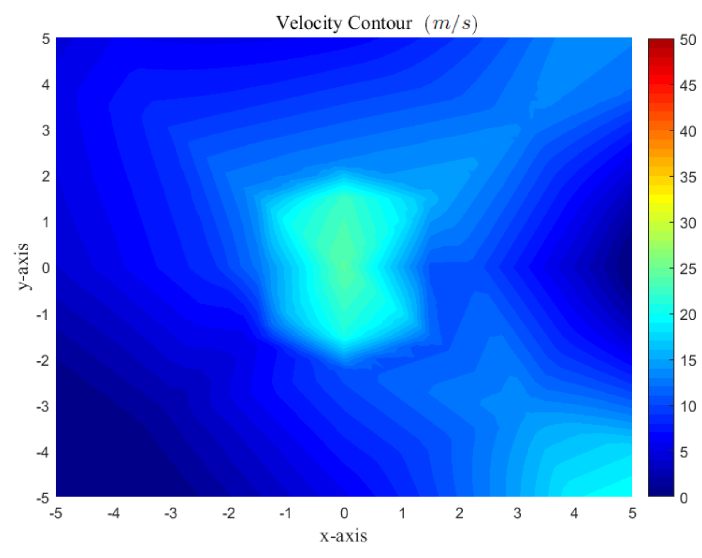

(c) Velocity contour plot at $\mathrm{t}=20 \mathrm{~ms}$.

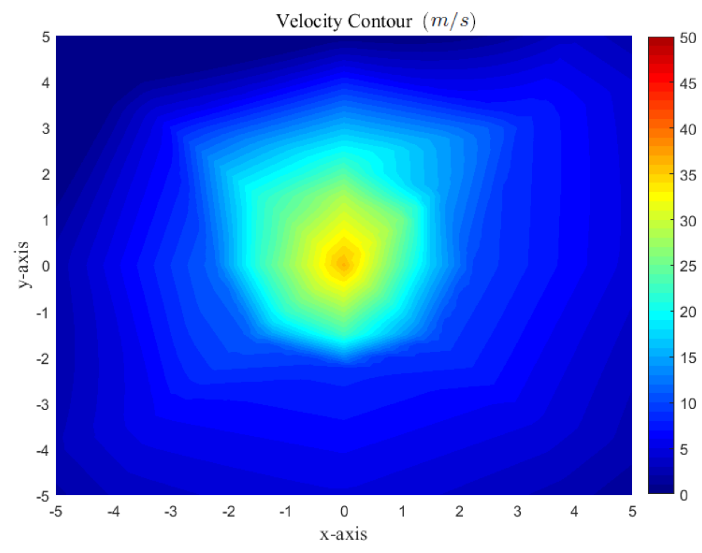

(e) Velocity contour plot at $\mathrm{t}=120 \mathrm{~ms}$.

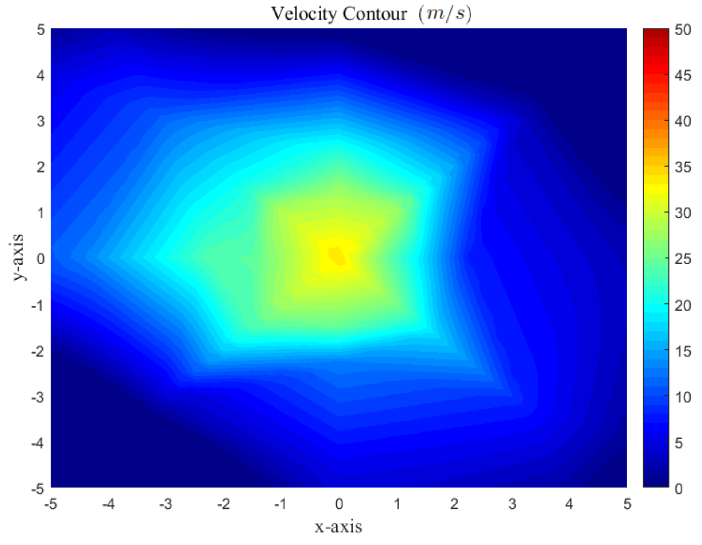

(b) Velocity contour plot at $\mathrm{t}=2 \mathrm{~ms}$.

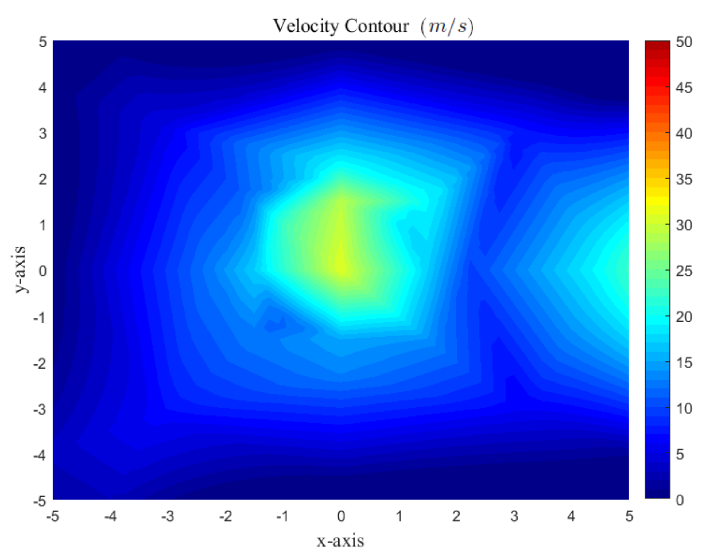

(d) Velcoity contour plot at t $=80 \mathrm{~ms}$.

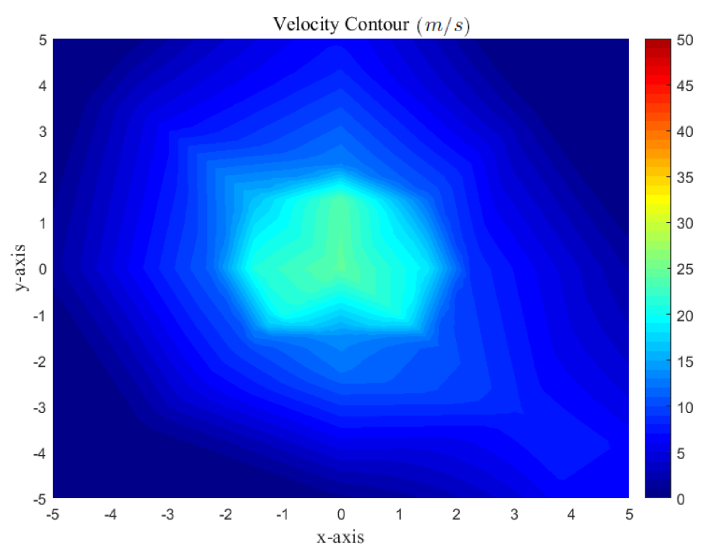

(f) Velocity contour plot at $\mathrm{t}=200 \mathrm{~ms}$.

Figure 5.8: Novo-Salbutamol HFA inhaler velocity contour plot from PDA measurement at $x=0 \mathrm{~mm}$. 


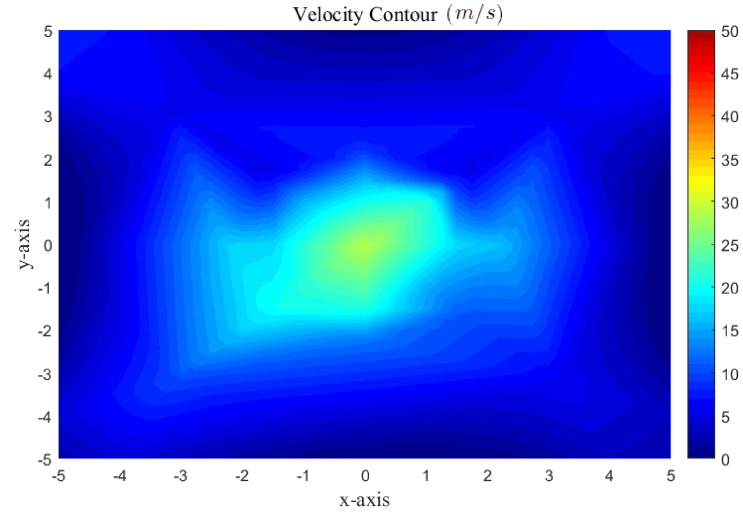

(a) Velocity contour plot at $\mathrm{t}=0 \mathrm{~ms}$.

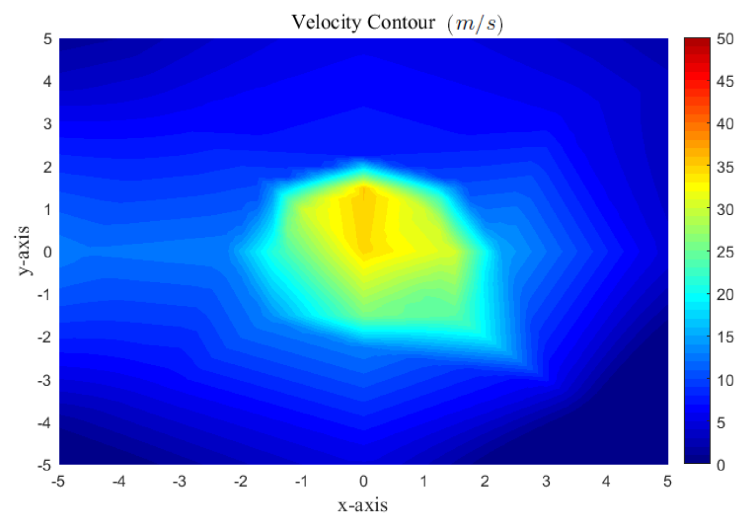

(c) Velocity contour plot at $\mathrm{t}=40 \mathrm{~ms}$.

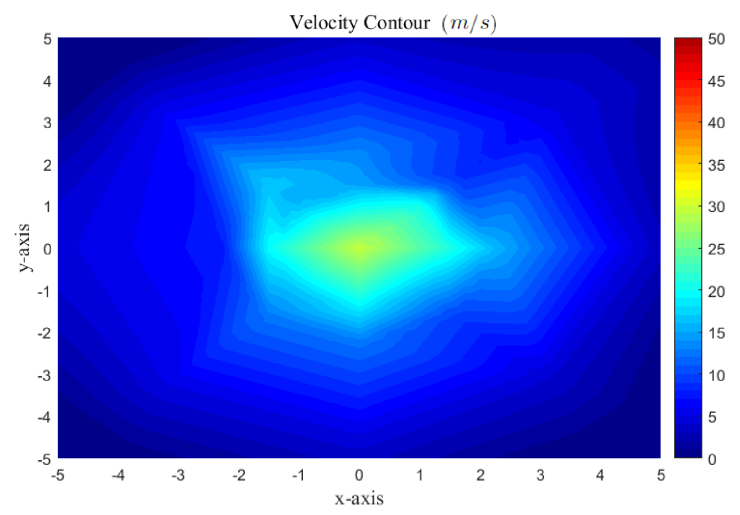

(e) Velocity contour plot at $\mathrm{t}=150 \mathrm{~ms}$.

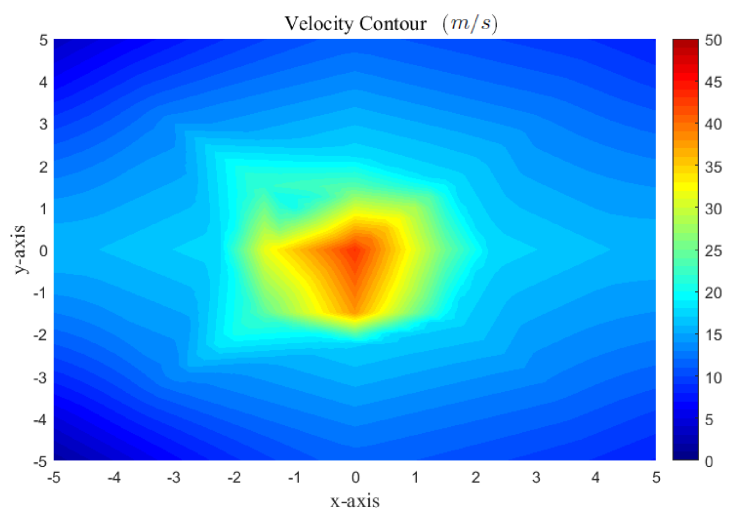

(b) Velocity contour plot at $\mathrm{t}=5 \mathrm{~ms}$.

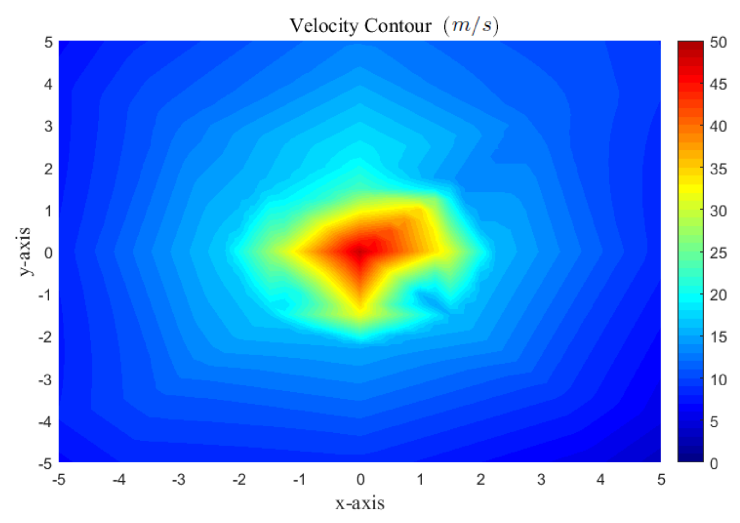

(d) Velcoity contour plot at $\mathrm{t}=100 \mathrm{~ms}$.

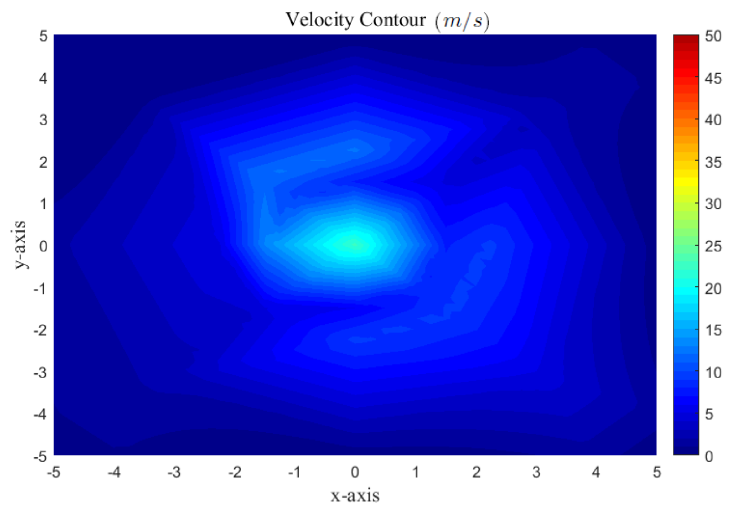

(f) Velocity contour plot at $t=200 \mathrm{~ms}$.

Figure 5.9: Airomir HFA inhaler velocity contour plot from PDA measurement at $x=0$ $m m$. 


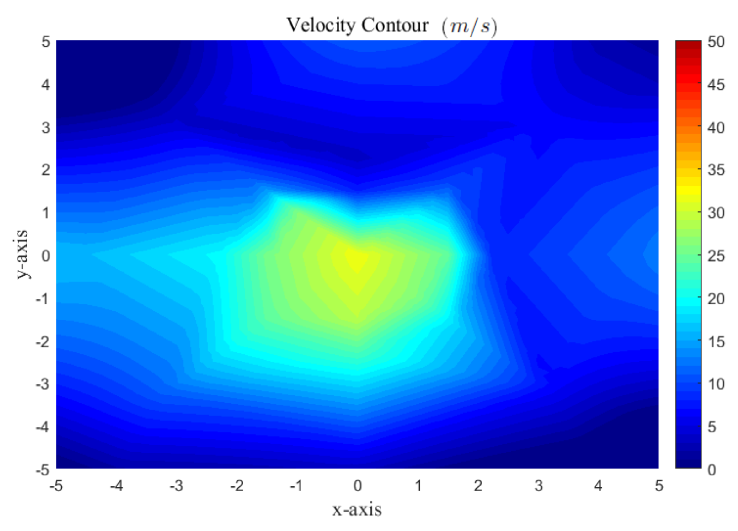

(a) Velocity contour plot at $\mathrm{t}=0 \mathrm{~ms}$.

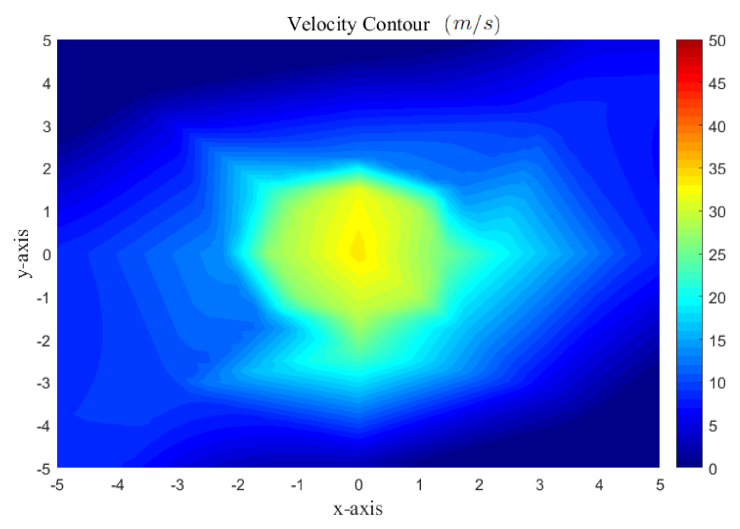

(c) Velocity contour plot at $\mathrm{t}=15 \mathrm{~ms}$.

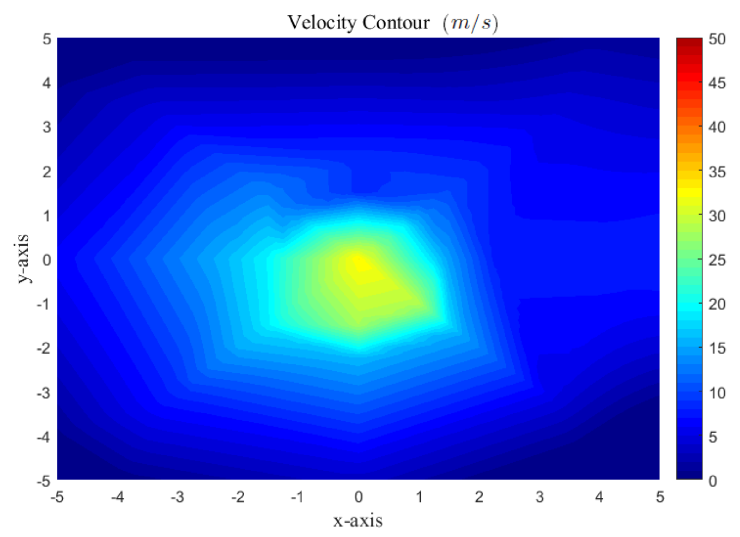

(e) Velocity contour plot at $\mathrm{t}=140 \mathrm{~ms}$.

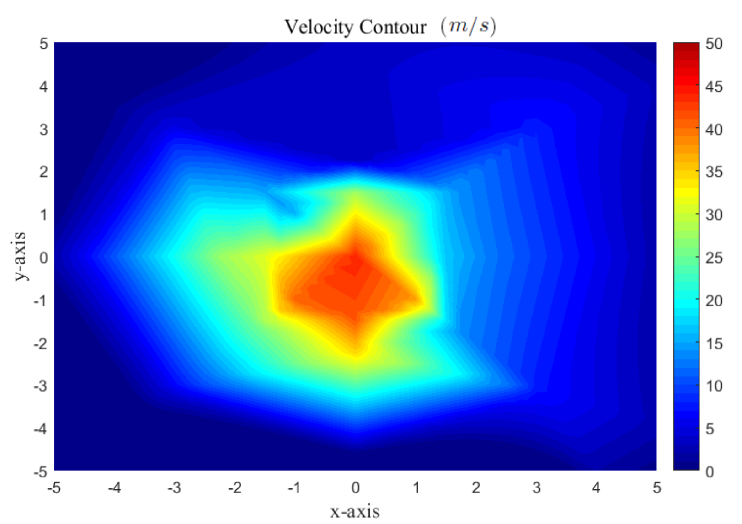

(b) Velocity contour plot at $\mathrm{t}=5 \mathrm{~ms}$.

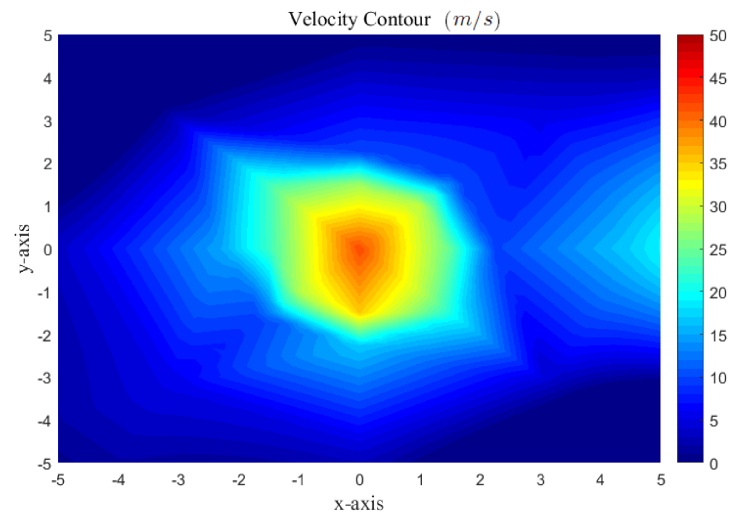

(d) Velcoity contour plot at $\mathrm{t}=100 \mathrm{~ms}$.

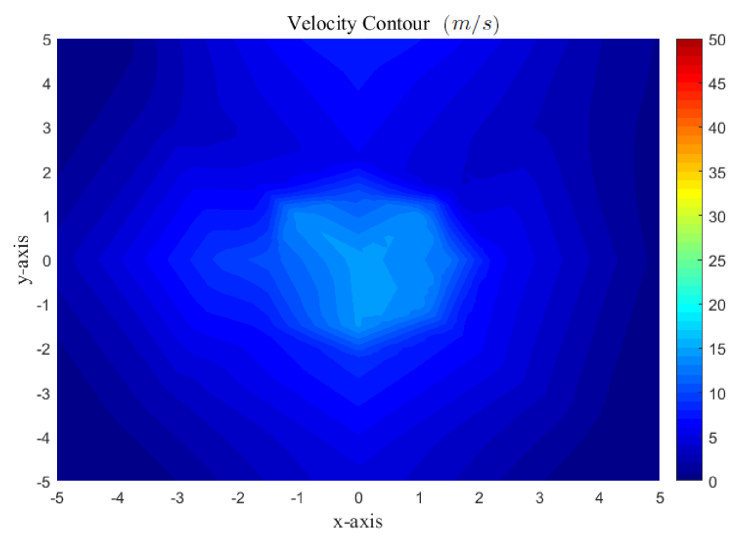

(f) Velocity contour plot at $\mathrm{t}=180 \mathrm{~ms}$.

Figure 5.10: Apo-Salvent CFC Free inhaler velocity contour plot from PDA measurement at $x=0 \mathrm{~mm}$. 
$12 \mathrm{~ms}$. Following this, the moving average diameter decreases with fluctuations until $90 \mathrm{~ms}$ where it is less than the mean $D_{10}$ and drops slightly below. Eventually, it rises slightly upwards in the very low spray density region at the end of the spray event.

Figure 5.11(b) shows the raw data for the particle diameters of five actuations for Novo-Salbutamol HFA, where the mean $D_{10}$ is about $2.78 \mu \mathrm{m}$. At the start of the actuation, the moving average diameter starts with a particle diameter of $2.25 \mu \mathrm{m}$ and goes above the mean at $3 \mathrm{~ms}$, then it keeps increasing until it reaches its peak value of $4.40 \mu \mathrm{m}$ at $17 \mathrm{~ms}$. The moving average diameter then declines with fluctuations above the mean until $93 \mathrm{~ms}$ where it falls slightly below the mean. At $93 \mathrm{~ms}$ it has another peak as a result of low intensity in the spray and it remains decreasing until the end of the actuation.

Also, the raw data for the particle diameters of five actuations of Airomir HFA is presented in Figure 5.11(c). The mean $D_{10}$ here is $2.55 \mu \mathrm{m}$ and the moving average diameter initially starts with a $1.53 \mu \mathrm{m}$ diameter at the beginning of the actuation in the high density region. Then, it reaches its peak at the end of high density area with a $3.53 \mu \mathrm{m}$ moving average diameter at a time of $7.44 \mathrm{~ms}$. After that, it remains higher than the mean until it reaches $110 \mathrm{~ms}$ and keeps declining until the end of the spray.

Similarly, particle diameter data of Apo-Salvent CFC Free is illustrated in Figure 5.11(d). The mean $D_{10}$ for this inhaler is $2.83 \mu \mathrm{m}$. The moving average diameter at first starts with $2.22 \mu \mathrm{m}$ and increases to its peak value at the end of the high density region at $6.91 \mathrm{~ms}$, with a moving average diameter of $3.98 \mu \mathrm{m}$. It remains above the mean until it crosses the mean at $118 \mathrm{~ms}$, where it declines at the end of the actuation period.

The particle diameter data for five actuations of all inhalers for the remaining stations at $75 \mathrm{~mm}$ and $100 \mathrm{~mm}$, is presented in Figures 5.12 and 5.13, respectively. Mostly, the moving average diameter is fluctuating around the mean with some peaks where there are low density areas such as found in the Novo-Salbutamol HFA at both stations 


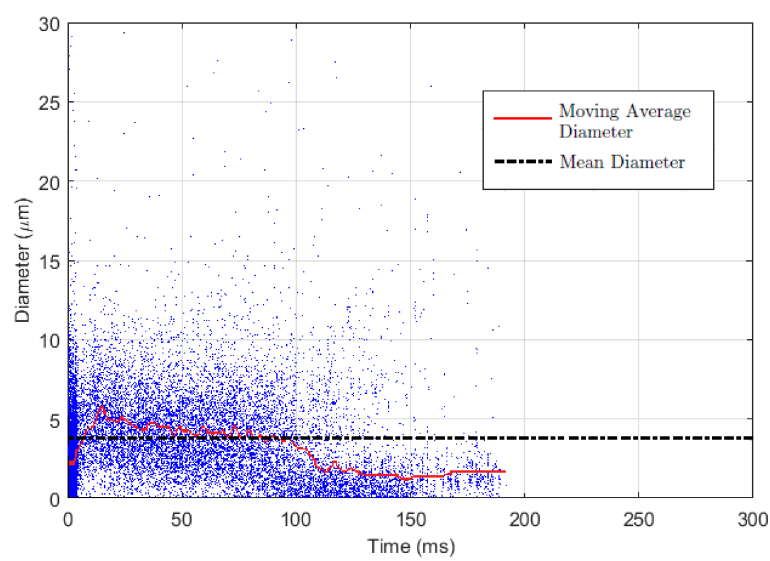

(a) For the Ventolin HFA inhaler.

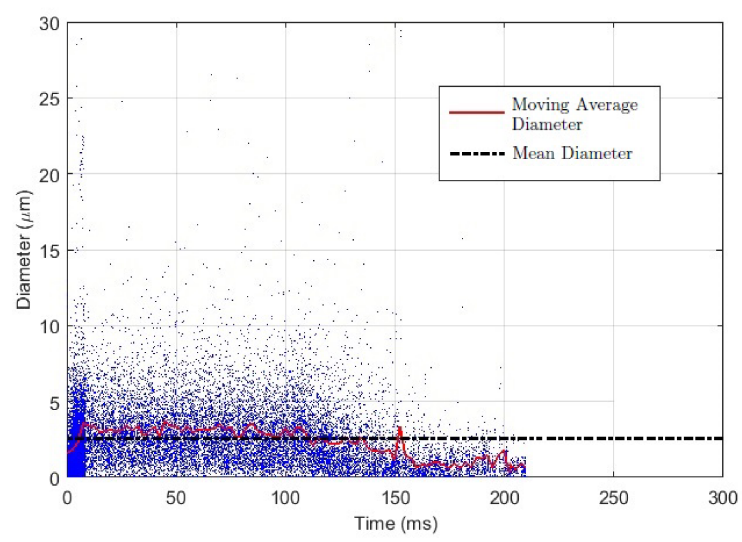

(c) For the Airomir HFA inhaler.

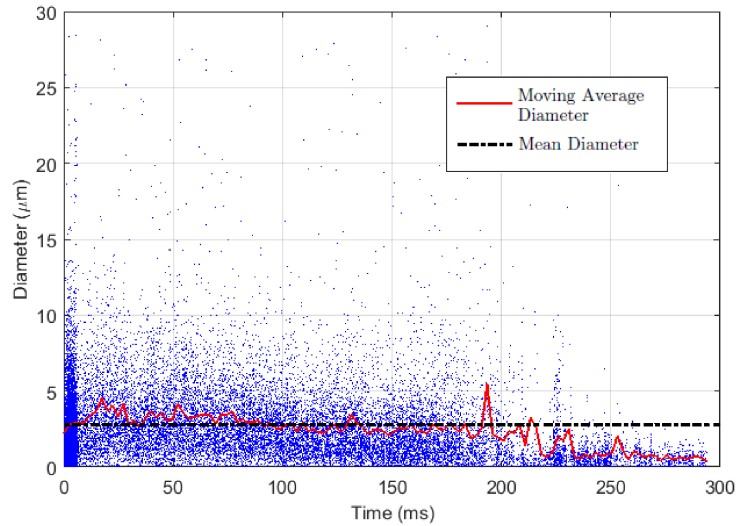

(b) For the Novo-Salbutamol HFA inhaler.

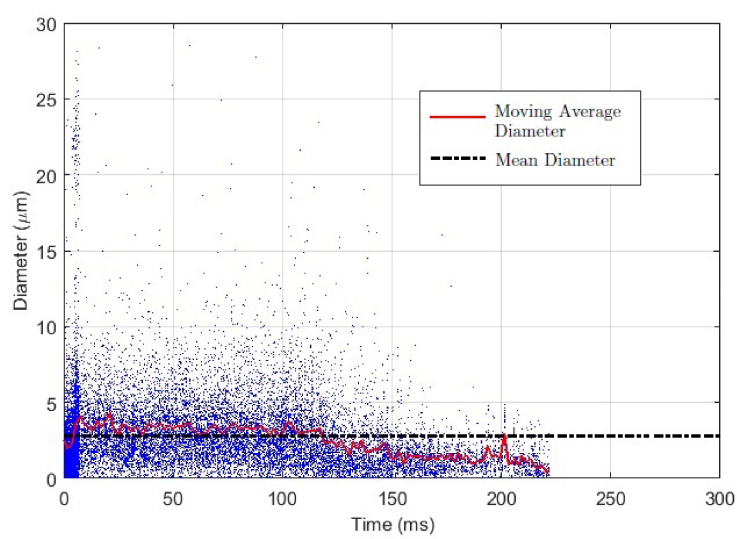

(d) For the Apo-Salvent CFC Free inhaler.

Figure 5.11: Raw data for the particle size for five actuations for each inhaler at $x=0$ $m m$. 


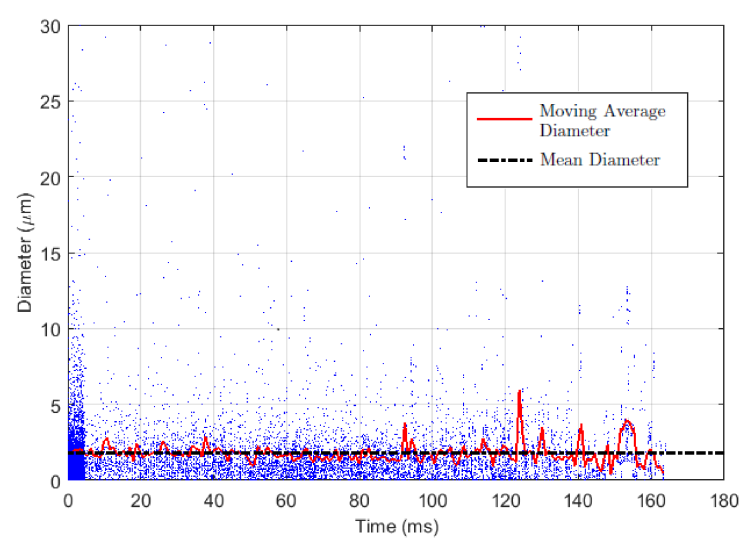

(a) For the Ventolin HFA inhaler.

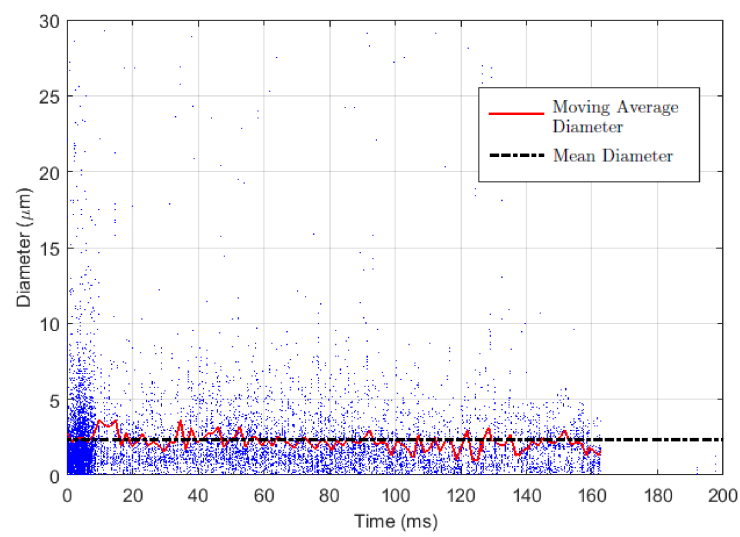

(c) For the Airomir HFA inhaler.

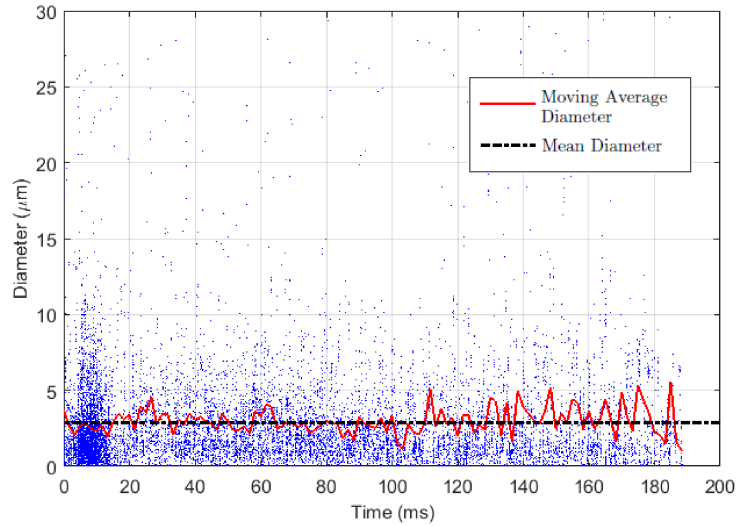

(b) For the Novo-Salbutamol HFA inhaler.

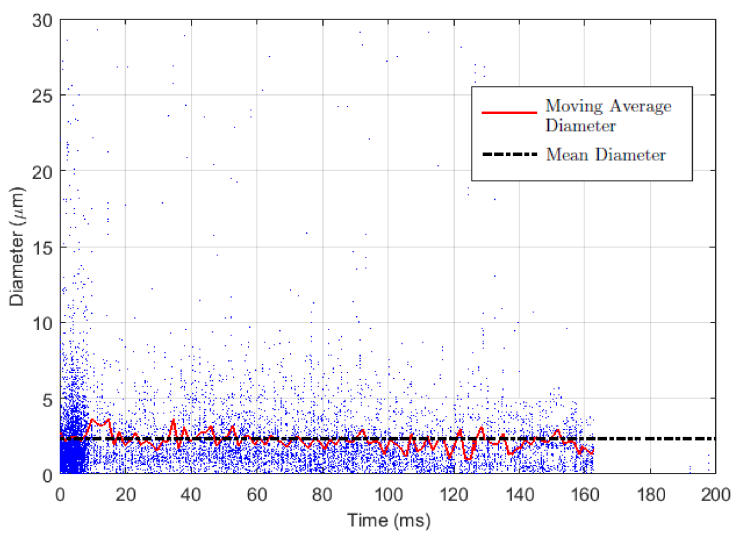

(d) For the Apo-Salvent CFC Free inhaler.

Figure 5.12: Raw data for the particle size for five actuations for each inhaler at $x=75$ $m m$.

at the end of the spray.

The Airomir HFA at $100 \mathrm{~mm}$ shows a larger high density region compared to other brands. The moving average diameter rises higher than the mean in this part (high density region), then it decreases below the mean at $18 \mathrm{~ms}$. Beyond this time, it remains fluctuating above the mean until it starts to decrease below the mean at $85.46 \mathrm{~ms}$ until the end of the spray event.

Finally, the particle diameter data for five actuations of Spiriva Respimat inhaler for 


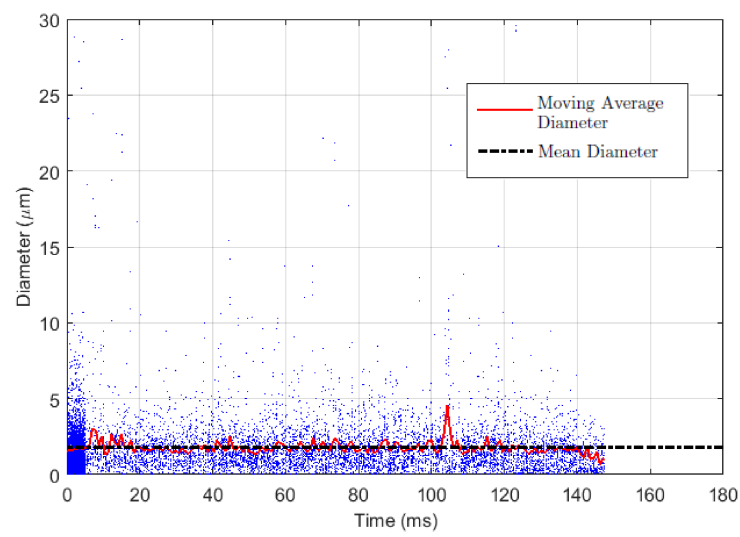

(a) For the Ventolin HFA inhaler.

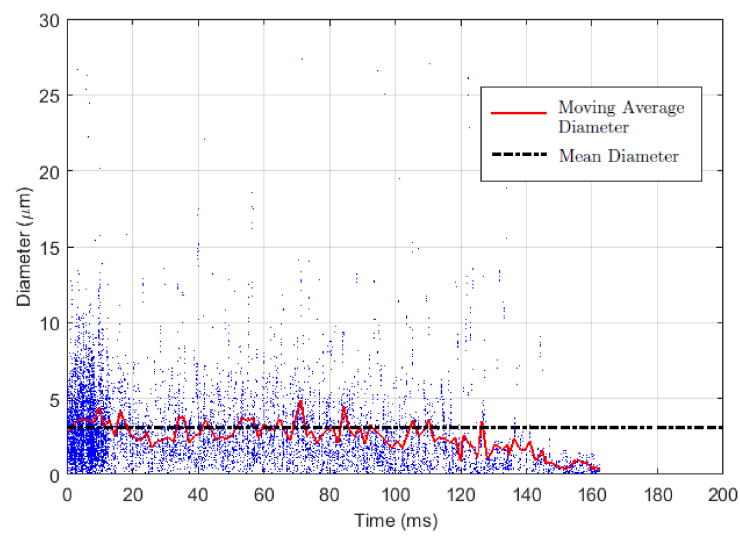

(c) For the Airomir HFA inhaler.

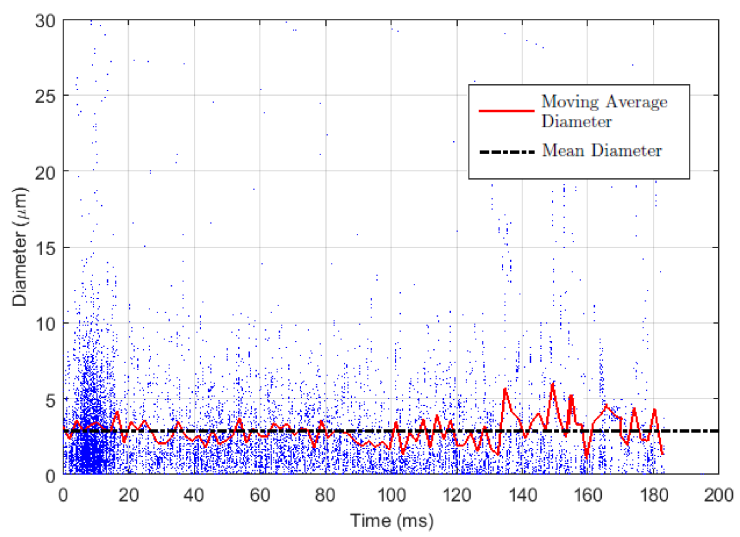

(b) For the Novo-Salbutamol HFA inhaler.

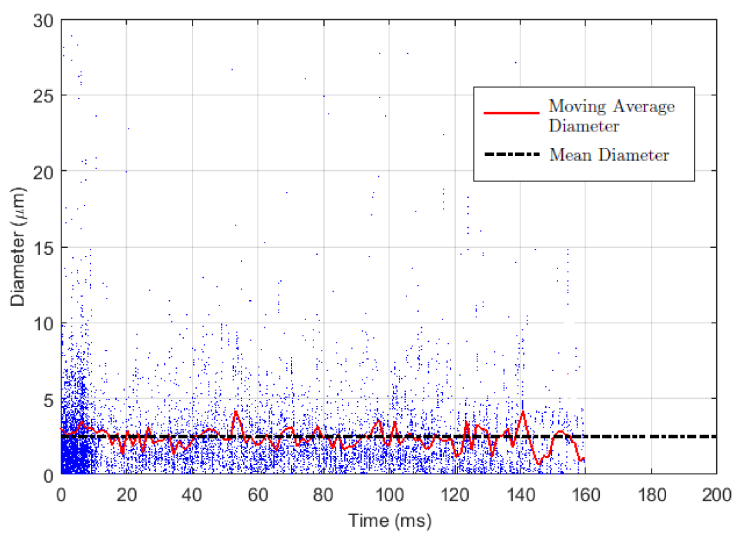

(d) For the Apo-Salvent CFC Free inhaler.

Figure 5.13: Raw data for the particle size for five actuations for each inhaler at $x=100$ $m m$. 
all locations is presented in Figure 5.14. its been observed in the first three locations -18.5, 0 and $75 \mathrm{~mm}$, that there is a large particle size is released at the end of the actuation, this is the dominant phenomenon in most of actuations, which could be due to the actuator and nozzle mechanism. However, in some cases event is not detected as in $100 \mathrm{~mm}$ locations. In addition, for both locations -18.5, $0 \mathrm{~mm}$ the average is below and close to the mean. At location $-18.5 \mathrm{~mm}$ starts with $5 \mu \mathrm{m}$ then it keeps lower than the mean until $1120 \mathrm{~ms}$ when it goes above the mean as a result of the large particles. For location $75 \mathrm{~mm}$ the moving average diameter starts below the mean and keeps fluctuating around it until the starts of the large particles phenomenon at the end of the actuation.

\subsubsection{Spatial analysis of the inhaler spray}

The spray issued from each tested pMDI is characterised spatially at each of the axial stations as discussed in the temporal analysis, in terms of the particle velocity and size. The goal is to examine the impact of the distance on particle velocity and size. A comparison is made to find the influence of the distance increment from the origin along the centerline on the horizontal axis for both of these parameters. This section also presents particle velocity and size at all locations from a single station in the cylindrical coordinate plane around the centerline.

\subsubsection{Velocity analysis of the pMDI spray}

Table 5.1 displays the measured mean particle velocity for the four pMDI products. Results for the pMDI products show that there is a variation in the mean particle velocities at the centerline, where in general the range is from $26.94 \mathrm{~m} / \mathrm{s}$ to $43.20 \mathrm{~m} / \mathrm{s}$ at $0 \mathrm{~mm}$, and from $5.49 \mathrm{~m} / \mathrm{s}$ to $12.22 \mathrm{~m} / \mathrm{s}$, at $100 \mathrm{~mm}$ away from the origin for all tested inhalers. The pMDI Ventolin HFA brand has the highest particle velocity at all three locations, while the other brands show an $18.04 \%$ decrease in the particle velocity 


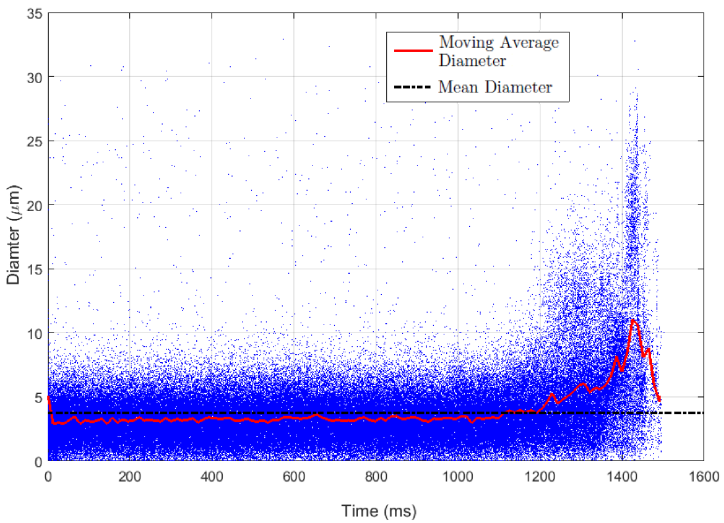

(a) At $x=-18.5 \mathrm{~mm}$.

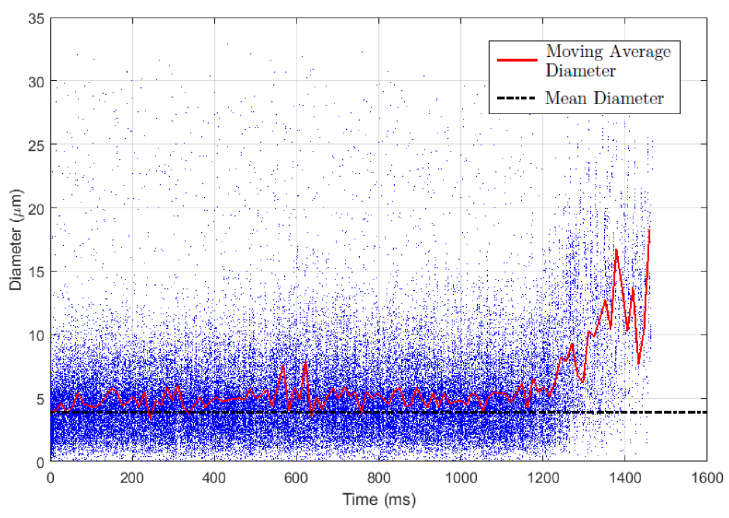

(c) At $x=75 \mathrm{~mm}$.

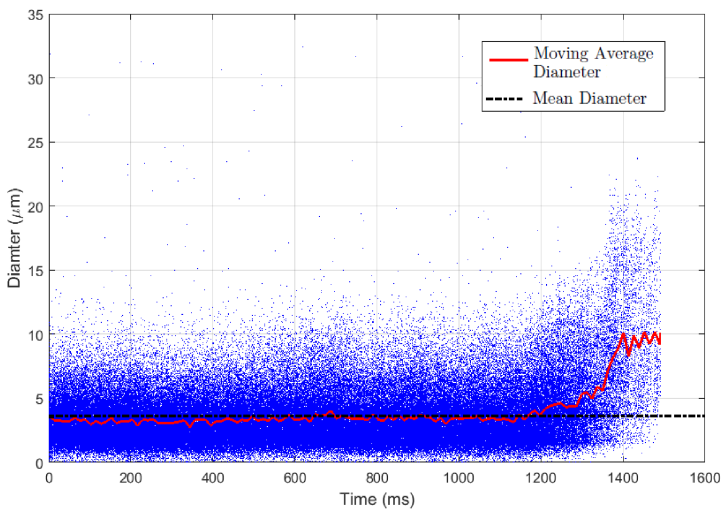

(b) At $x=0 \mathrm{~mm}$.

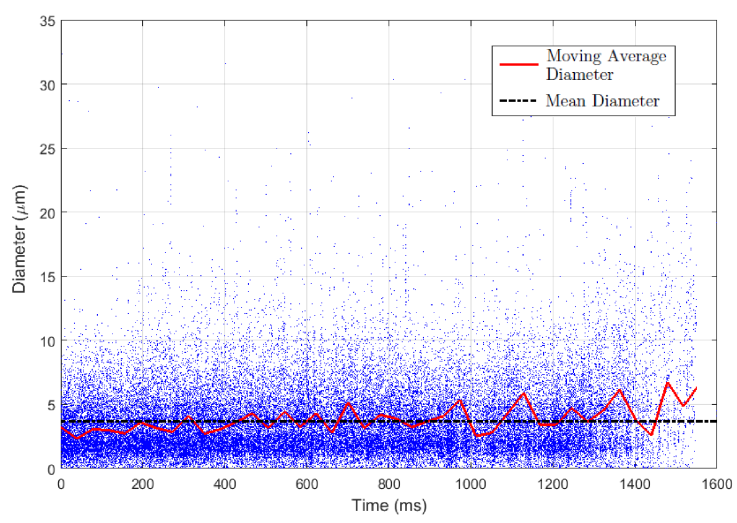

(d) At $x=100 \mathrm{~mm}$.

Figure 5.14: Raw data for the particle size for five actuations for Spiriva Respimat inhaler. 
between the Apo-Salvent CFC Free (highest of the two) and the Novo-Salbutamol HFA (the lowest) with particle velocities of $32.87 \mathrm{~m} / \mathrm{s}$ and $26.94 \mathrm{~m} / \mathrm{s}$, respectively at the $0 \mathrm{~mm}$ location. This reduction in the particle velocity along the centerline of the spray is caused by the evaporation rate of HFA propellants which reduces the kinetic energy quickly and diminishes the particles' velocity. Also, Liu et al. [17] reported that the geometry design (such as the valve and orifice) have a significant impact on particle velocity, rather than the propellant. Their study was to compare both velocity and size at two different locations along the center line, $30 \mathrm{~mm}$ and $60 \mathrm{~mm}$ from the mouthpiece edge. As an example, Albuterol CFC had a velocity of $15.10 \mathrm{~m} / \mathrm{s}$ and $10.80 \mathrm{~m} / \mathrm{s}$ for the two locations respectively with a decrease of $28.50 \%$. Whereas for Ventolin HFA it was $10.30 \mathrm{~m} / \mathrm{s}$ and $9.40 \mathrm{~m} / \mathrm{s}$ with a drop off of $8.00 \%$ only.

Table 5.1: Mean particle velocity measured at 0, 75, and $100 \mathrm{~mm}$ away from the origin along the centerline. (Units: $m / s$ ).

\begin{tabular}{|l|c|c|c|c|c|}
\hline & & & & \multicolumn{2}{|c|}{ Decrease \% } \\
\cline { 5 - 6 } pMDI products & i. At $0 \mathrm{~mm}$ & $i$. At $75 \mathrm{~mm}$ & $i$. At $100 \mathrm{~mm}$ & $i$ \& ii & $i$ \& iii \\
\hline Apo-Salvent CFC Free & 32.87 & 7.89 & 5.49 & 75.99 & 83.29 \\
\hline Airomir HFA & 32.40 & 8.66 & 7.44 & 73.27 & 77.03 \\
\hline Novo-Salbutamol HFA & 26.9 & 7.31 & 5.61 & 72.86 & 79.17 \\
\hline Ventolin HFA & 43.20 & 13.39 & 12.22 & 69.00 & 71.71 \\
\hline
\end{tabular}

A comparison of particle velocities at both locations, $0 \mathrm{~mm}$ and $75 \mathrm{~mm}$ highlights that the decrease in particle velocity in Apo-Salvent CFC Free is higher than the other products with a $75.99 \%$ drop. This decrease is relatively close to Airomir HFA with $73.27 \%$, while, for Ventolin HFA and Novo- Salbutamol HFA the decrease in particle velocity is $69.00 \%$ and $72.86 \%$, respectively. The decline in velocity between the second and third location is not significant in comparison, for any of the brands.

The results of the spatial analysis are presented in cylindrical-polar coordinates with 

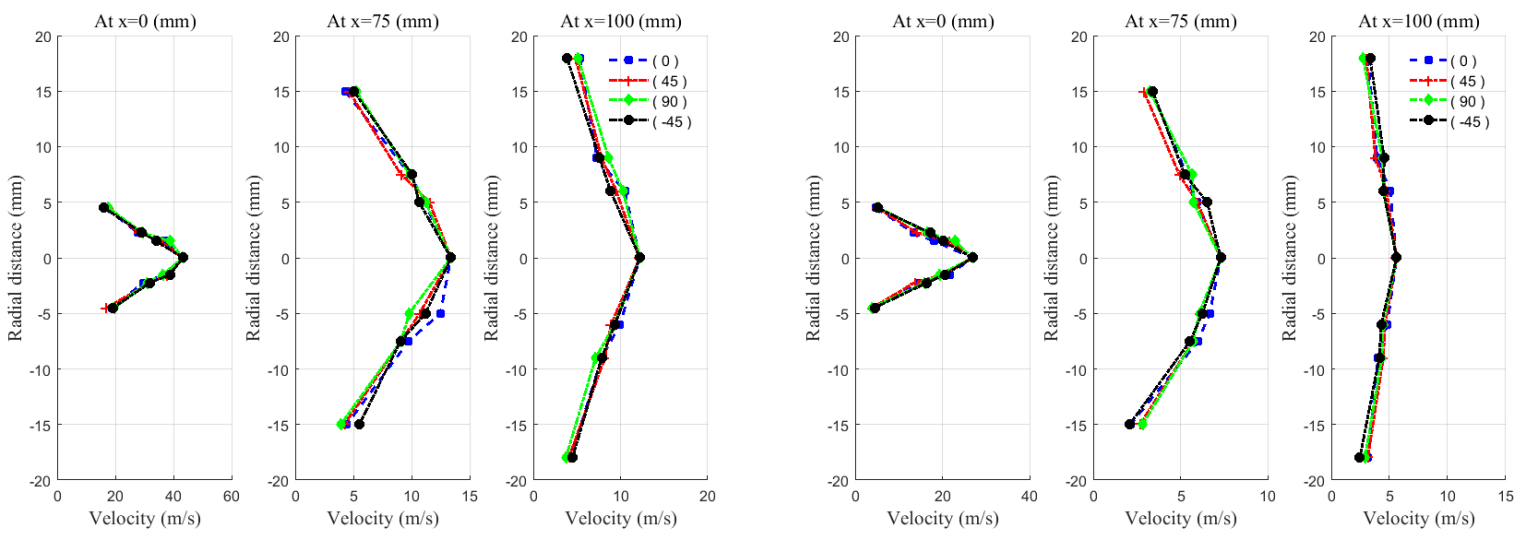

(a) For the Ventolin HFA inhaler.

(b) For the Novo-Salbutamol HFA inhaler.
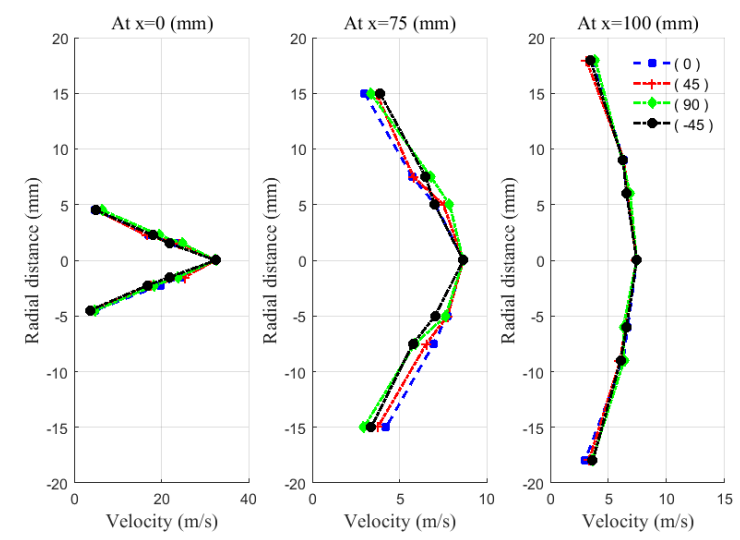

(c) For the Airomir HFA inhaler.
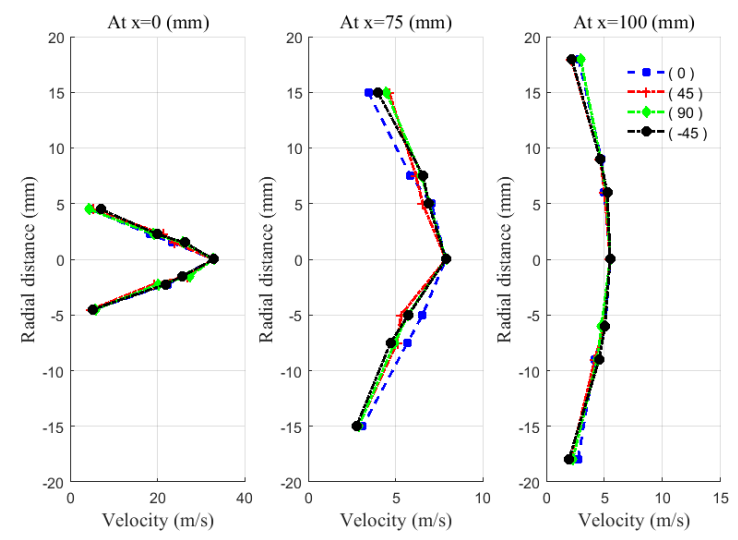

(d) For the Apo-Salvent CFC Free inhaler.

Figure 5.15: Velocity spatial analysis for pMDI inhalers.

radial profiles being presented along cylindrical planes of symmetry, i.e. $0^{\circ}, \pm 45^{\circ}$ and $90^{\circ}$, as shown in Figure 3.5. The radial profiles of the axial particle velocities at $x=0 \mathrm{~mm}$ are illustrated for each tested inhaler in Figure 5.15. The results show an asymmetrical profile for almost all pMDI inhalers and their peaks are the mean velocities as shown in Table 5.1. Ventolin HFA yields a higher velocity on the lower, while the Apo-Salvent CFC Free reveals a particle velocity profile with a peak value similar to the one obtained for Airomir HFA. The results for the remaining stations are also included in Figure 5.15. 
The mean velocity spatial analysis for Spiriva Respimat inhaler is shown in Table 5.2 , that the velocity decreases from $10.95 \mathrm{~m} / \mathrm{s}$ at the edge of the mouthpiece to 1.33 $\mathrm{m} / \mathrm{s}$ at the $100 \mathrm{~mm}$ location. A comparison is made between locations $-18.5 \mathrm{~mm}$ to other locations to calculate the drop in the velocity. This comparison shows a decrease of $58.72 \%$ in the 0 location and its almost equal at other locations. To evaluate the same reduction as pMDI at the same locations, that the decline in the velocity between point 0 and both points 75 and $100 \mathrm{~mm}$ show that both locations have an equal percentage in velocity drop with $70.13 \%$ and $70.57 \%$, respectively. This drop is less than all pMDI inhalers at both locations except for locations $75 \mathrm{~mm}$ its a bit higher than the Ventolin HFA.

Table 5.2: Mean particle velocity measured at $-18.5,0,75$, and $100 \mathrm{~mm}$ away from the origin along the centerline Spiriva Respimat inhaler. (Units: $\mathrm{m} / \mathrm{s}$ ).

\begin{tabular}{|c|c|c|c|c|c|c|c|c|}
\hline \multirow{2}{*}{.At } & ii.At & iii.At & iv.At & \multicolumn{5}{|c|}{ Decrease \% } \\
\cline { 5 - 9 }$-18.5 \mathrm{~mm}$ & $0 \mathrm{~mm}$ & $75 \mathrm{~mm}$ & $100 \mathrm{~mm}$ & $i \& i i$ & $i \&$ \&i & i \& iii & ii \& iii & i \& iv \\
\hline 10.95 & 4.52 & 1.35 & 1.33 & 58.72 & 87.67 & 87.85 & 70.13 & 70.57 \\
\hline
\end{tabular}

The results of the velocity spatial analysis for Spiriva Respimat inhaler are presented in both axis planes of symmetry, i.e. $0^{\circ}$ and $90^{\circ}$. Figure 5.16 shows the radial profiles of the axial particle velocities at locations 0,75 and $100 \mathrm{~mm}$. Again, the results produced an asymmetrical profile almost at 0 , and $75 \mathrm{~mm}$ locations and their peaks are the mean velocities. At location $0 \mathrm{~mm}$ the velocity is slightly lower on the axis $90^{\circ}$. An asymmetrical profile is observed at the last location for both axes as the velocity is higher on one axis in the upper region and vise-versa on the lower one.

\subsubsection{Particle diameter analysis of the inhaler spray}

In this study, the arithmetic mean diameter $\left(D_{10}\right)$, the volumetric mean diameter 

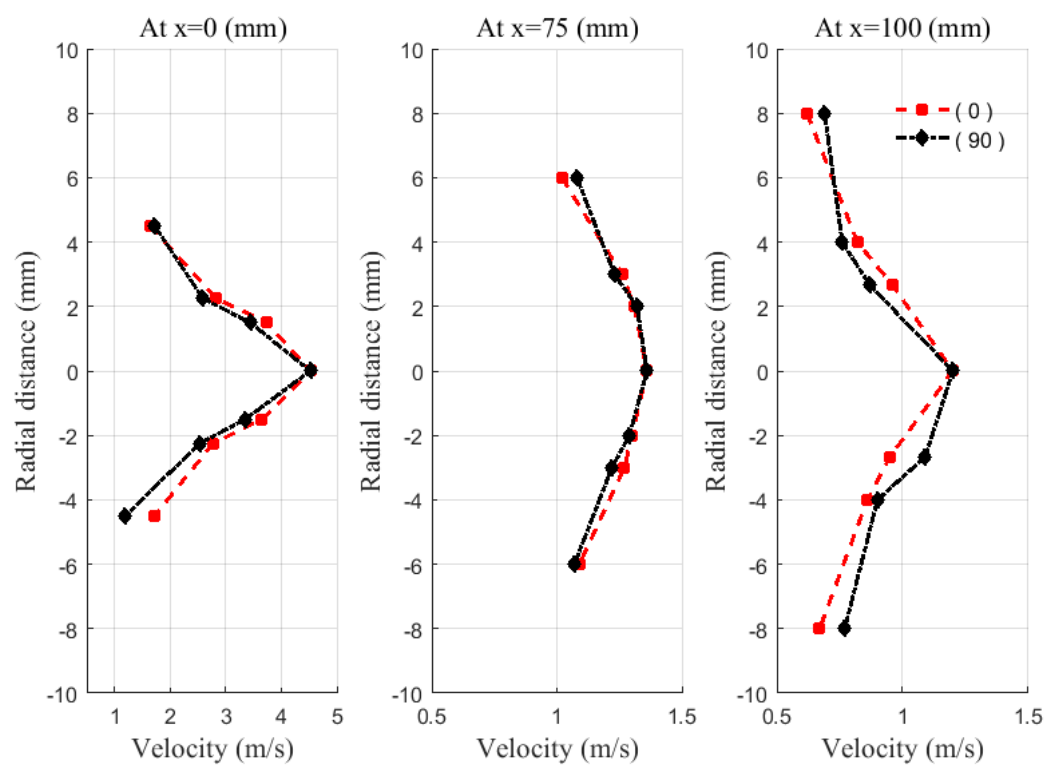

Figure 5.16: Velocity spatial analysis for Spiriva Respimat inhaler.

$\left(D_{30}\right)$, and volume-based median diameter $\left(D_{\mathrm{v} 50}\right)$ are studied at the same three locations, as shown in Figure 5.1. Arithmetic mean diameter $D_{10}$ is the simple average diameter of all the collected particles in a spray and is gained through Equation (2.1). The volume mean diameter $D_{30}$ is calculated from the mean of the particle volumes and is determined by Equation (2.2). Volume Median Diameter $D_{\mathrm{v} 50}$ is the volume averaged particle size; it characterizes the diameter of the droplets by $50 \%$ of the particles diameters, larger or smaller than the stated value of the total particle volume in the spray.

$D_{10}, D_{30}$, and $D_{\mathrm{v} 50}$ for the three locations along $x$-axis are all presented in Table 5.3. As can be seen for $D_{10}$ at the $0 \mathrm{~mm}$ station the values vary for all pMDIs, ranging from $2.55 \mu \mathrm{m}$ for Airomir HFA to $3.75 \mu \mathrm{m}$ for Ventolin HFA, whereas at the second station $(75 \mathrm{~mm})$ it is relatively close for all brands, except Ventolin HFA which had the smallest diameter with $1.84 \mu \mathrm{m}$. The reduction in the particle diameter for distances far away from the mouthpiece is due to the evaporation rate of the propellant. Also, for the Apo-Salvent CFC Free $D_{10}$ is $2.83 \mu m, 2.35 \mu m$, and $2.50 \mu m$ at the three stations 
respectively, whereas Airomir HFA's $D_{10}$ is $2.55 \mu \mathrm{m}$ at the $0 \mathrm{~mm}$ station, $2.76 \mu \mathrm{m}$ and $3.03 \mu \mathrm{m}$ at both the $75 \mathrm{~mm}$ and $100 \mathrm{~mm}$ locations. For Novo- Salbutamol HFA inhaler $D_{10}$ is $2.88 \mu \mathrm{m}$ at the second station, but ranged from $2.78 \mu \mathrm{m}$ to $2.84 \mu \mathrm{m}$ at the first and last stations. The reason why $D_{10}$ did not reduce for the tested inhalers (except for Ventolin HFA), is because the number of particles which are smaller than $D_{10}$ represented $62 \%$ to $68 \%$ of the total number of the released particles, while these only amounted to $57 \%$ for the Ventolin. This means that most of the small droplets would evaporate before reaching the next location. It can also be seen that $D_{30}$ is comparatively equal for the three locations, and that $D_{\mathrm{v} 50}$ has the smallest diameter compared to $D_{10}$ and $D_{30}$, where it ranges from $1.26 \mu \mathrm{m}$ to $3.20 \mu \mathrm{m}$.

The reason $D_{\mathrm{v} 50}$ is smaller than the others is that in a skewed distribution (as illustrated in Section 5.2.3), the mean $\left(D_{10}\right)$ lies closer to the direction of skew (the longer tail) relative to the median $\left(D_{\mathrm{v} 50}\right)$ [94]. In Liu et al. [17], $D_{\mathrm{v} 50}$ had the largest diameter for the particle sizes in both measurements by PDA and laser light scattering (LLS) systems which is based on the mechanism of light scattering by particles. In addition to that, their findings for the diameter ranges between both systems were considerably different, and it was suggested that the main differences in the measurement techniques could lead to the discrepancies in particle sizes.

Table 5.3: Particle size measurement for the four tested MDI inhalers $D_{10}, D_{30}$, and

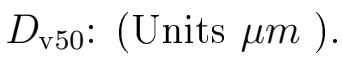

\begin{tabular}{|l|c|c|c|c|c|c|c|c|c|}
\hline \multirow{2}{*}{ pMDI products } & \multicolumn{3}{|c|}{$i$. At $0 \mathrm{~mm}$} & \multicolumn{3}{c|}{$i$. At $75 \mathrm{~mm}$} & \multicolumn{3}{c|}{$i$. At $100 \mathrm{~mm}$} \\
\cline { 2 - 11 } & $D_{10}$ & $D_{30}$ & $D_{\mathrm{v} 50}$ & $D_{10}$ & $D_{30}$ & $D_{\text {v50 }}$ & $D_{10}$ & $D_{30}$ & $D_{\text {v50 }}$ \\
\hline Apo-Salvent CFC Free & 2.83 & 5.17 & 2.24 & 2.35 & 5.51 & 1.61 & 2.50 & 5.30 & 1.76 \\
\hline Airomir HFA & 2.55 & 4.53 & 2.01 & 2.76 & 5.12 & 2.03 & 3.08 & 4.98 & 2.55 \\
\hline Novo-Salbutamol HFA & 2.78 & 5.23 & 2.11 & 2.88 & 6.16 & 1.94 & 2.84 & 5.87 & 1.89 \\
\hline Ventolin HFA & 3.75 & 5.76 & 3.20 & 1.84 & 5.01 & 1.26 & 1.80 & 4.08 & 1.41 \\
\hline
\end{tabular}


To determine whether there is a difference between two particles diameters within the inhalers, a T-test of two samples assuming equal variance in a Microsoft Excel data sheet is performed. Samples had to be taken form each of the two means being tested. In the test, a $5 \%$ level of significance, which equates to a $95 \%$ level of confidance, was selected. Therefore, the level of significance, often referred to as $\alpha$, in this test $\alpha=0.05$. Findings show that all particles diameters are statistically different for each brand of tested inhalers at all locations except for Apo-Salvent CFC Free and NovoSalbutamol HFA both at the $x=0 \mathrm{~mm}$ location.

The results of particle diameters of the spatial analysis, in terms of $D_{10}, D_{30}$, and $D_{\mathrm{v} 50}$, are displayed in Figures 5.17 and 5.18 for each tested puffer at $x=0 \mathrm{~mm}$, for all the cylindrical planes $0^{\circ}, \pm 45^{\circ}$, and $90^{\circ}$. The results obtained for $D_{10}$ (Figure 5.17) show a nearly monodisperse range of small particle diameters, as it varies from $3.67 \mu \mathrm{m}$ to $4.63 \mu \mathrm{m}$ for Ventolin HFA and from $2.63 \mu \mathrm{m}$ to $4.03 \mu \mathrm{m}$ for Novo-Salbutamol HFA with a slight variation in the last location on the top side for all the cylindrical planes. Also, it varies from $2.45 \mu \mathrm{m}$ to $3.67 \mu \mathrm{m}$ for Airomir HFA, and from $2.31 \mu \mathrm{m}$ to $3.40 \mu \mathrm{m}$ for Apo-Salvent CFC Free.

The $D_{30}$, and $D_{\text {v } 50}$ radial profiles shown in Figure 5.18 for all inhalers reveal that the maximum size for $D_{30}$, (which is the second profiles with higher values) occurred on the last locations around the $x$ - axis at $(0, \pm 4.5, \theta)$. It was also observed that for Apo-Salvent CFC Free $D_{30}$ is higher on the top side comparing to the opposite sides at both locations, that is $(0,2.25, \theta)$, and $(0,1.5, \theta)$. As for $D_{\mathrm{v} 50}$ in general, at most locations it stays around, or higher than the size at location $(0,0, \theta)$, except for the Ventolin HFA, where its profile shows that it is slightly lower on the bottom side with an mean value of $2.08 \mu \mathrm{m}$. This is $35 \%$ lower than the mean particle diameters at location $(0,0, \theta)$. Although on the top side the first location at $(0,1.5, \theta)$ except for $(0,1.5,-45)$ reveal the maximum in particle diameters at this station. The results for both other locations at $x=75 \mathrm{~mm}$ and $x=100 \mathrm{~mm}$, regarding their particle diameters 

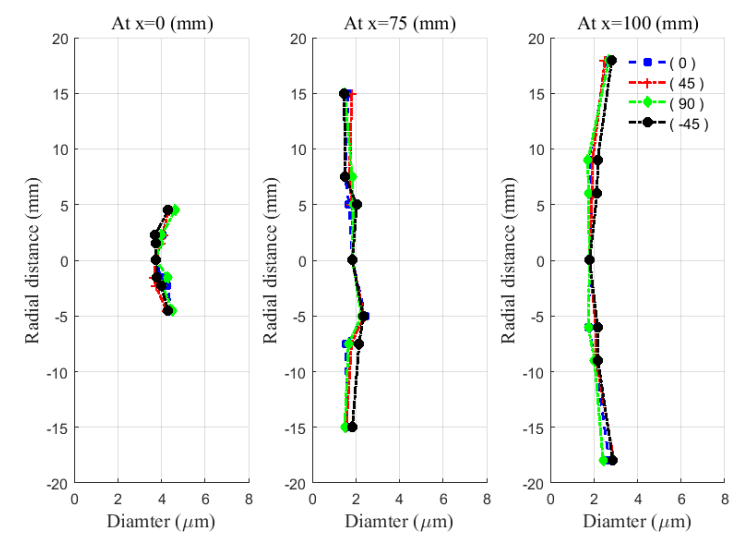

(a) For the Ventolin HFA inhaler.
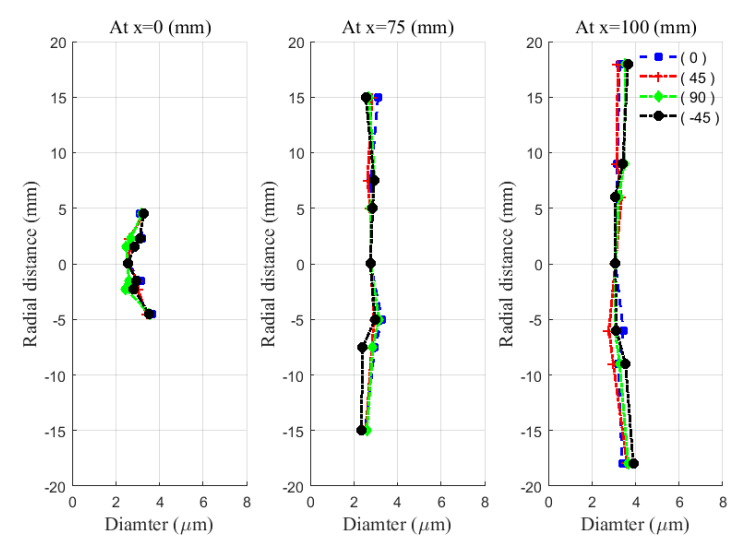

(c) For the Airomir HFA inhaler.
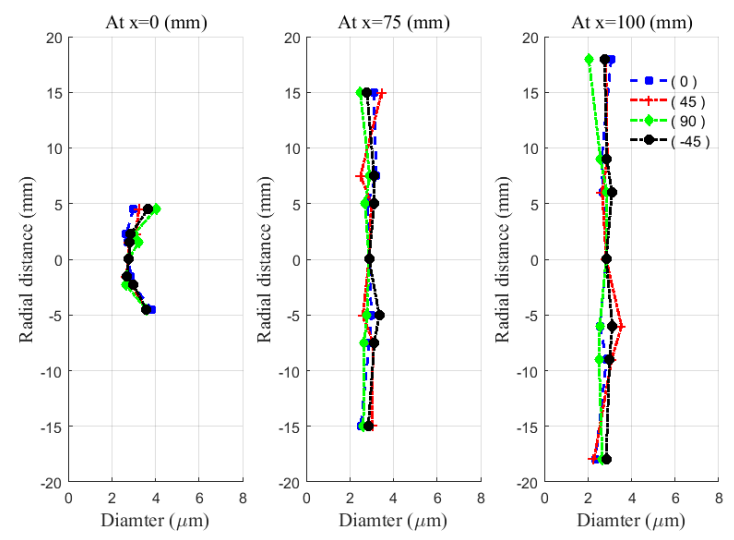

(b) For the Novo-Salbutamol HFA inhaler.
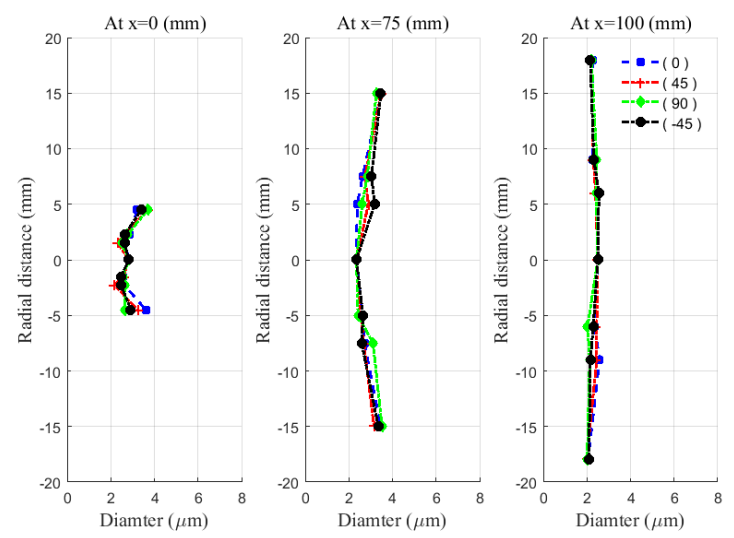

(d) For the Apo-Salvent CFC Free inhaler.

Figure 5.17: $D_{10}$ profiles for particles diameter spatial analysis. 

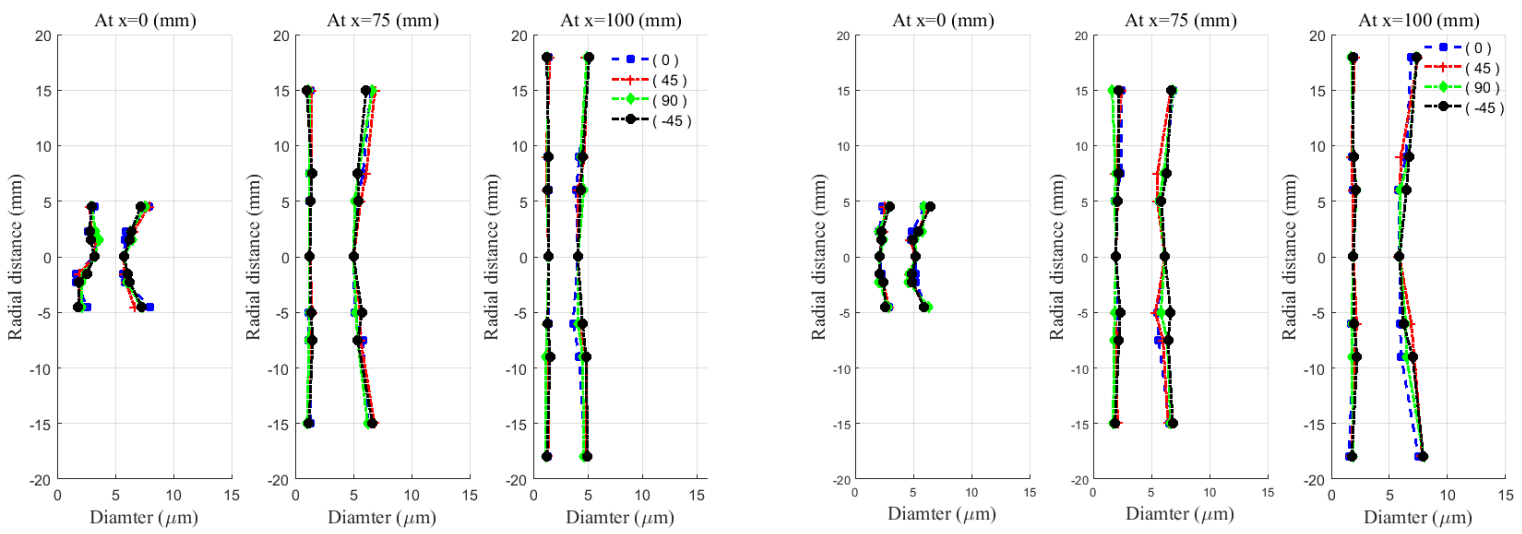

(a) For the Ventolin HFA inhaler.

(b) For the Novo-Salbutamol HFA inhaler.
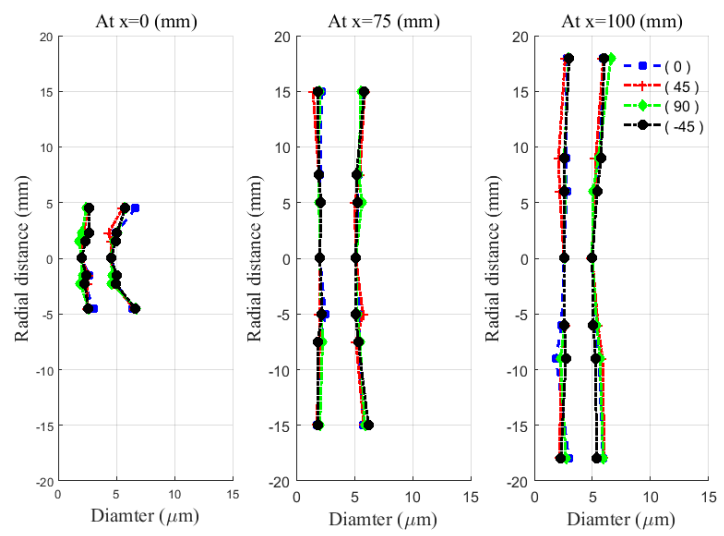

(c) For the Airomir HFA inhaler.
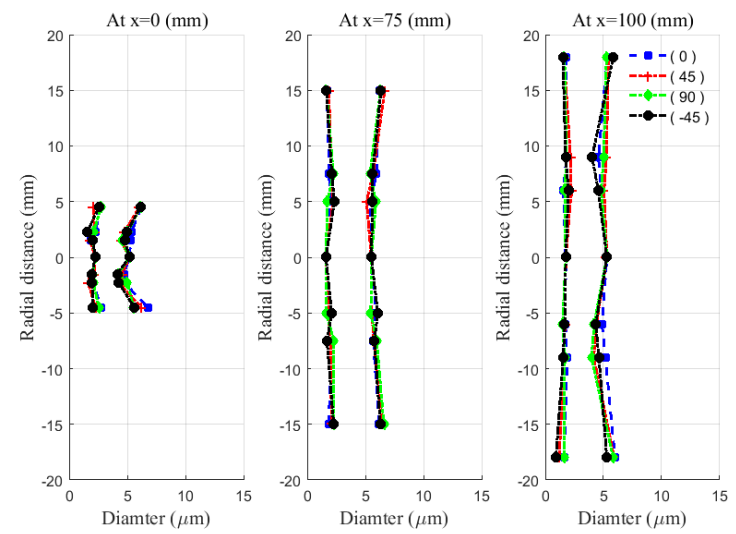

(d) For the Apo-Salvent CFC Free inhaler.

Figure 5.18: $D_{30}$, and $D_{\mathrm{v} 50}$ profiles for particles diameter spatial analysis.

spatial analysis can seen in Figure 5.18.

$D_{10}, D_{30}$ and $D_{\mathrm{v} 50}$ for the four locations along x -axis for Spiriva Respimat inhaler are all presented in Table 5.4. As can be seen for $D_{10}$ values varied for all locations, ranging from $3.97 \mu \mathrm{m}$ at the point $-18.5 \mathrm{~mm}$ to $3.67 \mu \mathrm{m}$ at $100 \mathrm{~mm}$ location. Also, as illustrated in Table 5.4 there is an increase in the particle size in the last point, as it decreases from the point $-18.5 \mathrm{~mm}$ then increases at point $100 \mathrm{~mm}$ as it is observed in the pMDI inhalers in Table 5.1. Also, all $D_{10}, D_{30}$ and $D_{\mathrm{v} 50}$ for Spiriva Respimat inhaler show higher values in comparison to pMDI inhalers for all three locations. 
Table 5.4: Particle size measurement for the four Spiriva Respimat inhaler $D_{10}, D_{30}$, and $D_{\mathrm{v} 50}$ : (Units $\mu m$ ).

\begin{tabular}{|c|c|c|c|c|c|c|c|c|c|c|c|}
\hline \multicolumn{3}{|c|}{$i$. At $-18.5 \mathrm{~mm}$} & \multicolumn{3}{c|}{$i i$. At $0 \mathrm{~mm}$} & \multicolumn{3}{c|}{$i i i$. At $75 \mathrm{~mm}$} & \multicolumn{3}{c|}{$i v$. At $100 \mathrm{~mm}$} \\
\hline$D_{10}$ & $D_{30}$ & $D_{\mathrm{v} 50}$ & $D_{10}$ & $D_{30}$ & $D_{\text {v } 50}$ & $D_{10}$ & $D_{30}$ & $D_{\text {v50 }}$ & $D_{10}$ & $D_{30}$ & $D_{\text {v } 50}$ \\
\hline 3.97 & 6.48 & 3.41 & 3.58 & 4.97 & 3.11 & 3.32 & 5.80 & 2.59 & 3.67 & 5.75 & 2.92 \\
\hline
\end{tabular}
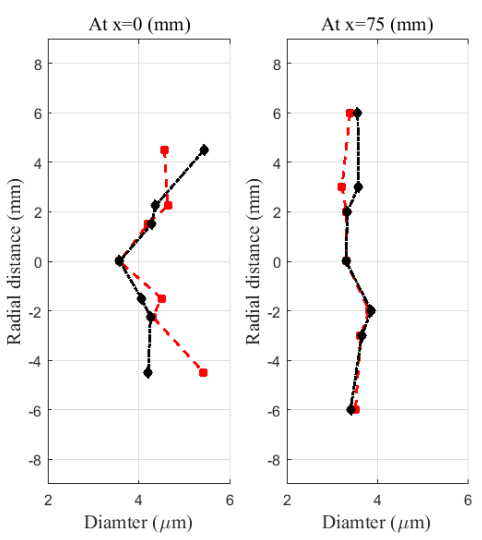

(a) $D_{10}$ profiles for particles diameter.
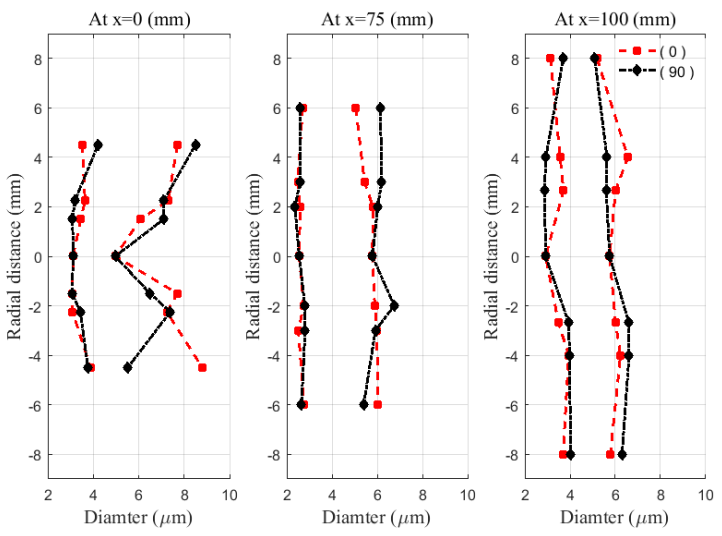

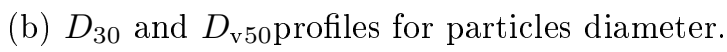

Figure 5.19: Particle diameter spatial analysis for Spiriva Respimat inhaler.

The results of particles diameters of the spatial analysis for Spiriva Respimat inhaler, in terms of $D_{10}, D_{30}$ and $D_{\mathrm{v} 50}$, are displayed in Figures 5.19, for the 0, 75, $100 \mathrm{~mm}$ locations. For $D_{10}$ its mostly higher than the mean value in the center point $(0,0)$ for all locations, whereas, for $D_{30}$ is close to the mean value in the center point $(0,0)$ at all locations. However, $D_{\mathrm{v} 50}$ is varied in location $0 \mathrm{~mm}$ as it goes higher than mean value in the center point and increases as the distance increase from the center point, and it keeps close to the mean value in the center point for both locations 75, and $100 \mathrm{~mm}$.

\subsubsection{Particle size distributions}

The study of particle size distributions from pMDI sprays indicates the frequency of occurrence of particles with different sizes. In this study, distribution fitting techniques 
were applied on measured data to get the most appropriate fitting. There are many functions that can be used to calculate probability density functions (PDFs). In this case three PDFs were selected for the data analysis: (i) Rosin-Rammler, (ii) Log-Normal, and (iii) Nakagami.

The Rosin-Rammler probability distribution function was originally applied to describe the size distribution of crushed coal, although it is successfully used to compute the size distribution in many fields including pharmaceutical aerosols. This function can be expressed as [95]:

$$
f(x ; \lambda, k)=\left\{\begin{array}{cl}
\frac{k}{\lambda}(x / \lambda)^{k-1} e^{-(x / \lambda)^{k}} & , x \geq 0 \\
0 & , x<0
\end{array}\right.
$$

Where $x$ is the independent variable, $\lambda$ is the mean diameter, and $k$ is the distribution spread.

The log-normal probability distribution function is as follows [95]:

$$
f(x ; \mu, \sigma)=\frac{1}{x \sigma \sqrt{2 \pi}} e^{-(\ln x-\mu)^{2} / 2 \sigma^{2}} \quad, x>0
$$

Where $\sigma$ represents the shape parameter (the standard deviation of the log of the distribution), and $\mu$ is the scale parameter (the mean of the distribution).

The Nakagami distribution function is given by Equation (5.5):

$$
f(x ; m, \Omega)=\frac{2 m^{m} x^{2 m-1}}{\Gamma(m) \Omega^{m}} \exp \left(\frac{m}{\Omega} x^{2}\right)
$$

Where $\Gamma$ is the gamma function, $\Omega$ the scaling parameter and $m$ is the Nakagami parameter which is related to the shape of the Nakagami distribution. This function is also used to characterize the particle size distribution in liquid water content during fog conditions [96]. 
The histogram of size distributions for each inhaler obtained at $x=0 \mathrm{~mm}$ from the origin are shown in Figure 5.20. The size distribution data was used as the source data file to calculate the three probability distribution functions and the lines in the figures result from curve fitting for these functions. As it can be seen from Figure 5.20, the Rosin-Rammler PDF (the red line) gives the best fitting of the data compared to the other two PDFs. Also, log-normal PDF shows poor curve fitting. In an earlier study experimental data was gained through the use of an Andersen eight-stage cascade impactor by Dunbar and Hickey [97]. The investigation was conducted to find the best distribution and fitting method for pMDI with CFC formulation and the study found that log-normal PDF showed the best curve fitting. In the current study, however, the Rosin-Rammler PDF demonstrated better curve fitting among the tested PDFs which is in disagreement to the earlier study. Table 5.5 presents the optimum parameters for each PDF function for all pMDI inhalers.

Table 5.5: Optimum parameters for three selected PDFs for tested pMDIs.

\begin{tabular}{|l|c|c|c|c|c|c|}
\hline \multirow{2}{*}{ pMDI products } & \multicolumn{2}{|c|}{ Rosin-Rammler } & \multicolumn{2}{c|}{ Log-normal } & \multicolumn{2}{c|}{ Nakagami } \\
\cline { 2 - 7 } & $\lambda$ & $k$ & $\mu$ & $\sigma$ & $m$ & $\Omega$ \\
\hline Apo-Salvent CFC Free & 4.07 & 1.31 & 0.96 & 1.00 & 0.52 & 22.45 \\
\hline Airomir HFA & 2.72 & 1.20 & 0.54 & 1.01 & 0.46 & 11.78 \\
\hline Novo-Salbutamol HFA & 3.05 & 1.23 & 0.68 & 0.94 & .048 & 14.71 \\
\hline Ventolin HFA & 2.94 & 1.16 & 0.61 & 1.01 & 0.43 & 14.84 \\
\hline
\end{tabular}

Also to find how the experimental data will follow any of the selected PDFs, a series of tests are carried out by changing the bin size to see how this will affect the curve fitting. Again, Rosin-Rammler shows a better curve fitting. In addition, a Chi-squared test is used to find a measure of the goodness of fit of a mathematical model to an experimental data set. The measured data is divided into $K$ bins and the Chi-squared 


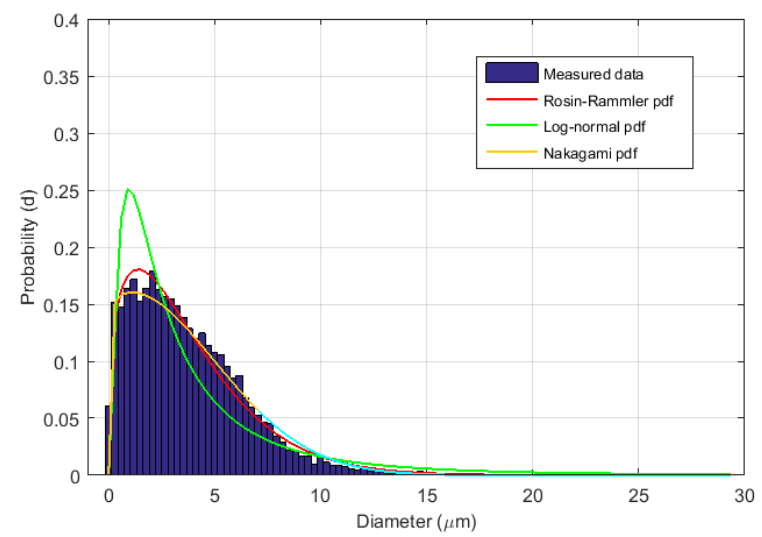

(a) For the Ventolin HFA inhaler.

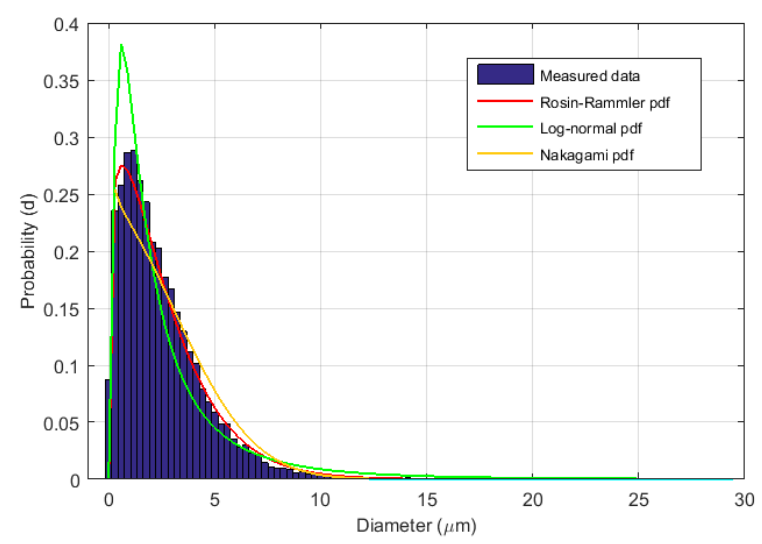

(c) For the Airomir HFA inhaler.

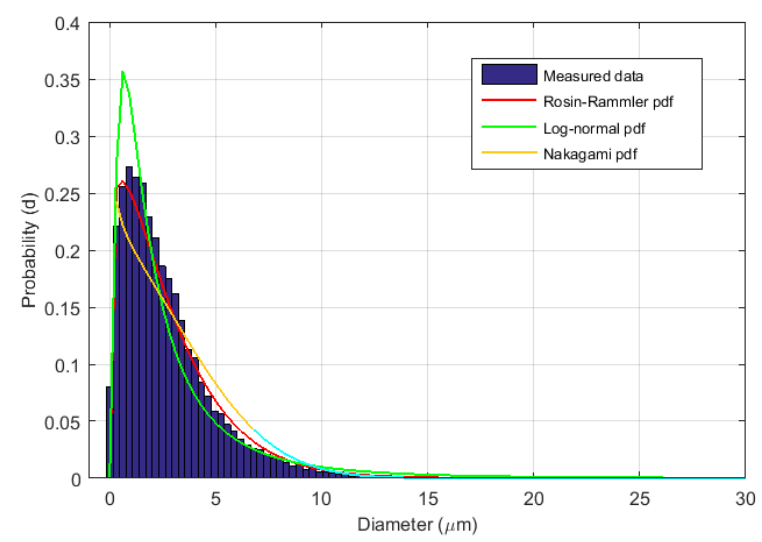

(b) For the Novo-Salbutamol HFA inhaler.

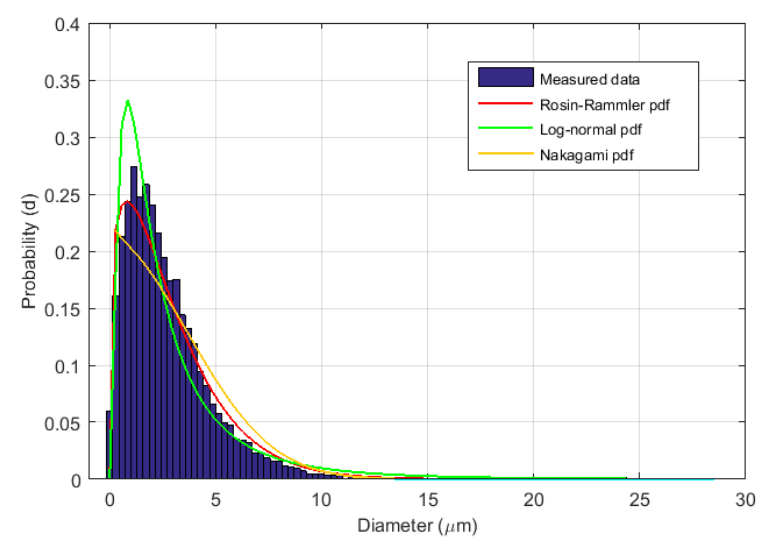

(d) For the Apo-Salvent CFC Free inhaler.

Figure 5.20: Histogram of the experimental data of particles diameter with curve fitting of probability distribution functions at $0(\mathrm{~mm})$. 
statistic test is defined as [98]:

$$
\chi^{2}=\frac{\sum_{j}\left(n_{j}-n_{j}^{\prime}\right)^{2}}{n_{j}^{\prime}} \quad j=1,2, \ldots K
$$

Where $n_{j}$ is the observed frequency for bin $j$ and $n_{j}^{\prime}$ is the expected frequency for bin $j$.

Considering all tested PDFs, the Rosin-Rammler presents a value of $P\left(\chi^{2}\right)<0.05$ for all tested inhalers, indicating a very strong measure of fit to the mentioned distribution. The goodness of fit test is performed using EasyFit Professional (MathWave Technologies) software, which calculates the goodness of fit between multiple PDFs and shows how they rank against each other. For the selected PDFs the Rosin-Rammler PDF ranks 1 for all tested pMDI inhalers, whereas the Nakagami PDF does not fit the experimental data. The goodness of fit parameters used for the three selected PDFs for all pMDI inhalers are similar for both the Rosin-Rammler and the Log-normal, where the degree of freedom, $P$ - Value , and critical value is $14.00,0.00$, and 23.68 respectively.

Therefore, from these results the Rosin-Rammler PDF is used in this study for further analysis, to find-out the particle diameters distributions in time intervals for the tested inhalers at the $x=0 \mathrm{~mm}$ station. Thus, six time intervals are used for the entire spray duration. Figure 5.21(a) displays the particles diameter distributions of six time intervals for Ventolin HFA. The first three curves (the red, the blue, and green curves) represent the time from the start of the actuation to a time less than $90 \mathrm{~ms}$ which is half of the spray time. It illustrates that most of the large particles are released within this time with a probability of $20 \%$ or less. This phenomenon is observed at the second and the third time intervals for both Airomir HFA and Apo-Salvent CFC Free in Figure 5.21(c), Figure 5.21(b) and Figure 5.21(d). Whereas, in Novo- Salbutamol HFA it is only occurring at the second time interval. Ventolin HFA produces fewer small 


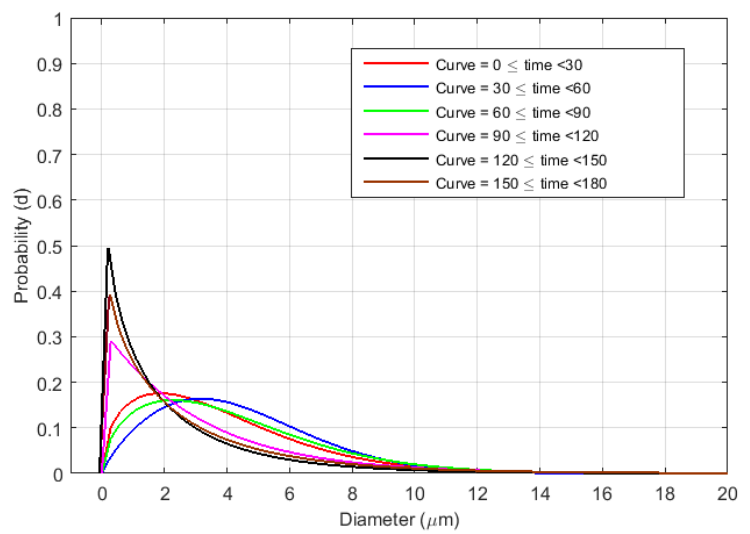

(a) For the Ventolin HFA inhaler.

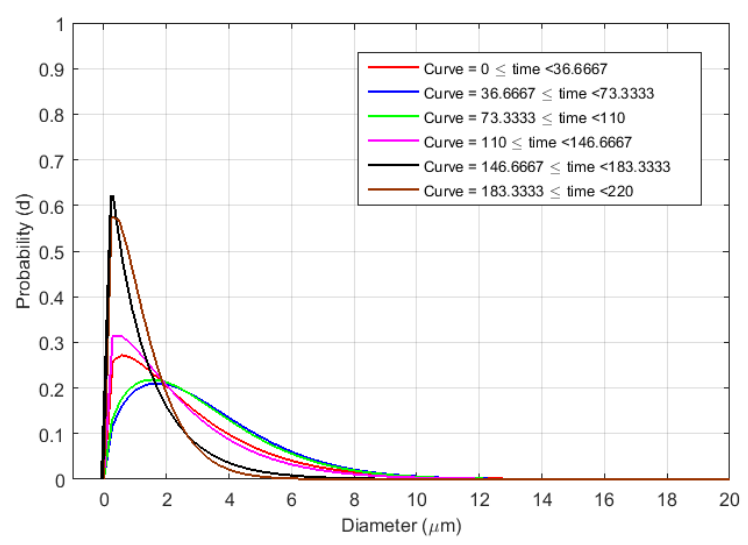

(c) For the Airomir HFA inhaler.

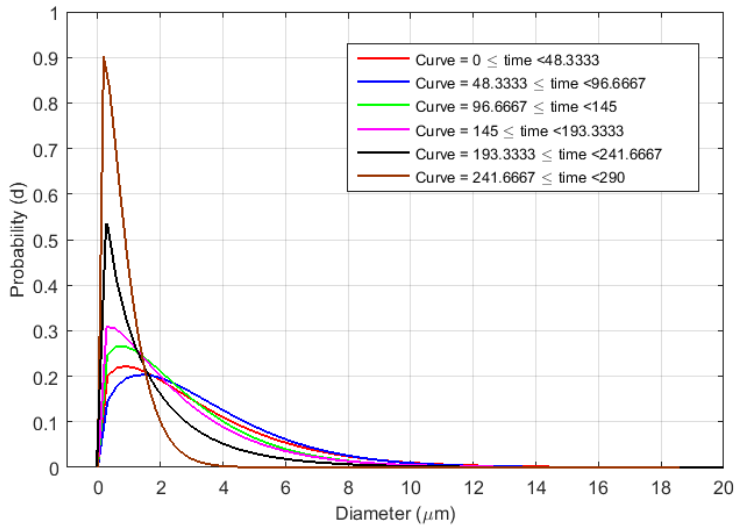

(b) For the Novo-Salbutamol HFA inhaler.

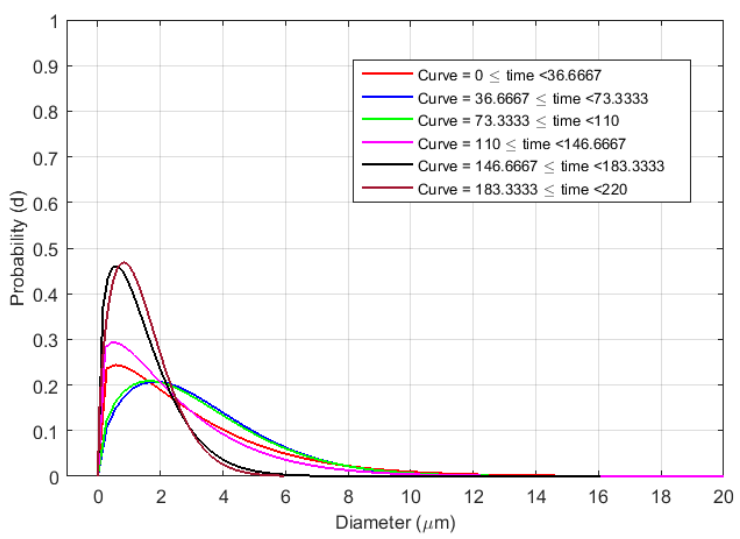

(d) For the Apo-Salvent CFC Free inhaler.

Figure 5.21: The particles diameter distributions of six time intervals for each inhaler at $0(\mathrm{~mm})$.

particles compared to the other inhalers, while Novo- Salbutamol HFA yields only small particles in the sixth time interval of the time window as illustrated in Figure 5.21(b).

Figure 5.22 shows the histogram of the experimental data of particles diameter with curve fitting of probability distribution functions for Spiriva Respimat inhaler for both locations -18.5 and $0 \mathrm{~mm}$. In contract to pMDI inhalers, log-normal PDF shows best curve fitting for Spiriva Respimat inhaler at both locations, but the histogram skews in the $-18.5 \mathrm{~mm}$ location. Table 5.6 presents the optimum parameters for each PDF for 


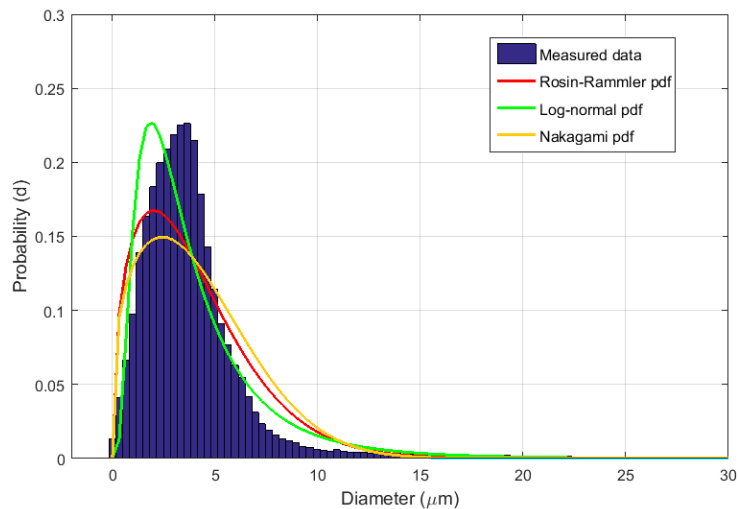

(a) At $-18.5 \mathrm{~mm}$.

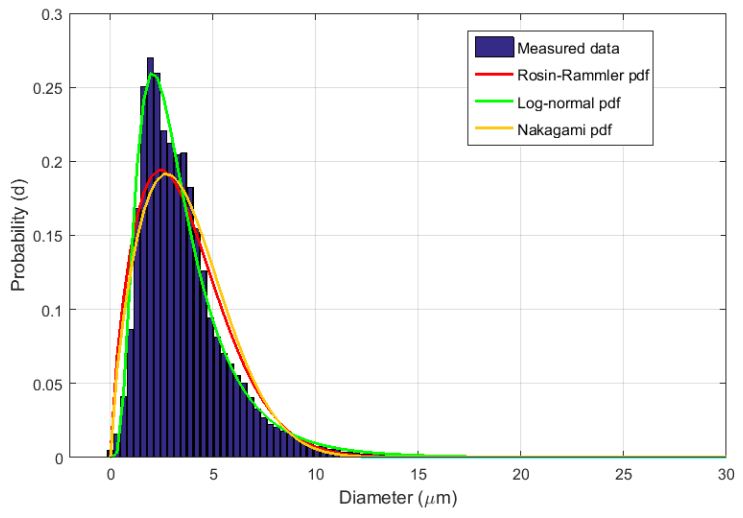

(b) At $0 \mathrm{~mm}$.

Figure 5.22: Histogram of the experimental data of particles diameter with curve fitting of PDFs for Spiriva Respimat inhaler.

both locations.

Table 5.6: Optimum parameters for three selected PDFs for Spiriva Respimat inhaler.

\begin{tabular}{|l|c|c|c|c|c|c|}
\hline \multirow{2}{*}{ Location } & \multicolumn{2}{|c|}{ Rosin-Rammler } & \multicolumn{2}{c|}{ Log-normal } & \multicolumn{2}{c|}{ Nakagami } \\
\cline { 2 - 7 } & $\lambda$ & $k$ & $\mu$ & $\sigma$ & $m$ & $\Omega$ \\
\hline At $-18.5 \mathrm{~mm}$ & 4.41 & 1.46 & 1.14 & 0.72 & 0.66 & 225.37 \\
\hline At $0 \mathrm{~mm}$ & 4.04 & 1.72 & 1.10 & 0.61 & 0.85 & 17.94 \\
\hline
\end{tabular}

Also, the log-normal PDF is used in for further analysis for Spiriva Respimat inhaler, with the same process used to the Rosin-Rammler PDF for pMDI inhalers. Figure 5.23 shows that most large particles are released at the last time intervals (brown curve) at a time around $1300 \mathrm{~ms}$ for both locations -18.5 and $0 \mathrm{~mm}$. This event is in contrast with the tested pMDIs where small particles are emitted at the end of the actuation at $0 \mathrm{~mm}$ location. 


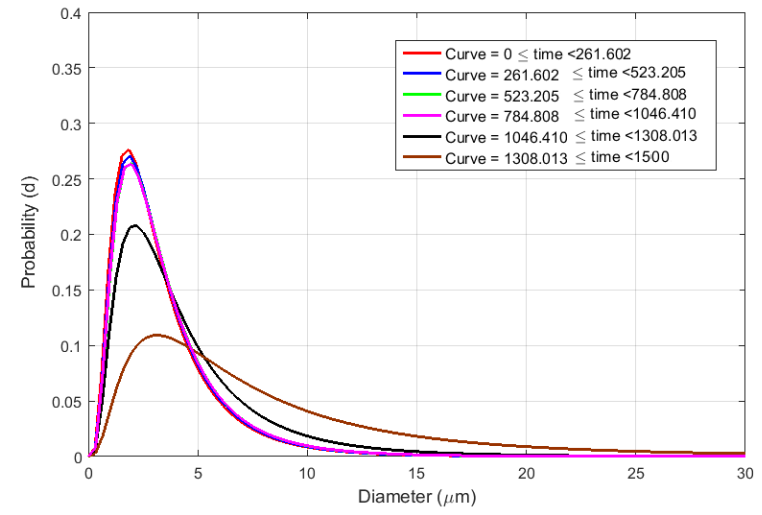

(a) At $-18.5 \mathrm{~mm}$.

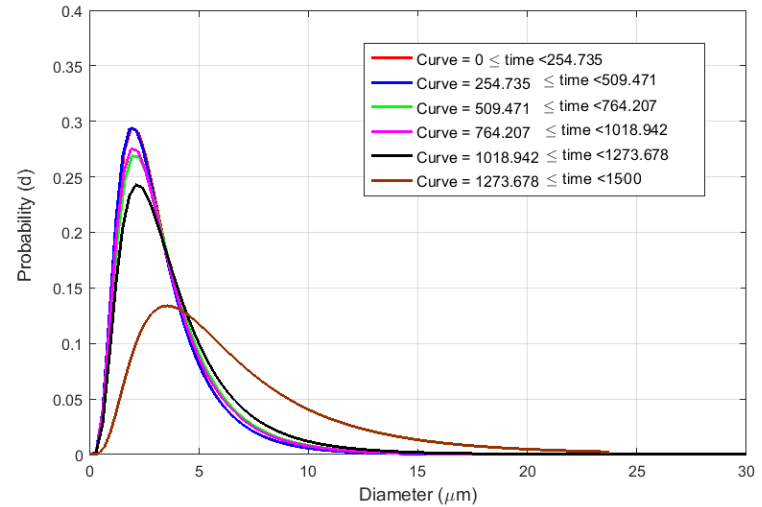

(b) At $0 \mathrm{~mm}$.

Figure 5.23: The particles diameter log-normal PDF of six-time intervals for Spiriva Respimat inhaler. (Time unit: $(m s)$ ).

\subsubsection{Aerosol Characterization using ACI}

\subsubsection{Data and Statistical Analyses}

The drug deposition results were expressed as a percentage of the total recorded medication. The mean $\pm \mathrm{SD}$ of the drug deposition is evaluated from at least 5 repeats for each test case. To ensure the reported results are statistically significant, t-tests were conducted for the drug deposited in the lungs. For each test case, a t-value was calculated using the following Equation:

$$
t=\frac{\bar{X}-\mu}{S / \sqrt{n}}
$$

where $n$ is the number of samples, $\bar{X}$ is the sample mean, and $S$ is the sample standard deviation. The hypothesis variable $\mu$ is assumed to be the expected percentage drug delivered to the lung $(\mu=40 \%)$. $P$-Values of $<0.05$ were considered statistically significant. Calculations are done with MATLAB R2014b software.

To characterize the particle size distribution (PSD), the mass median aerodynamic diameter (MMAD) and the geometric standard deviation (GSD) are used. MMAD, 
which is based on a model that assumes a log-normal distribution of the particle size and mass, is determined at the diameter corresponding to the 50th mass percentile $\left(D_{50}\right)$. GSD describes the spread of the data in the distribution and is evaluated under the log-normal distribution using the following Equation.

$$
\mathrm{GSD}=D_{84} / D_{50}=D_{50} / D_{16}=\left(D_{84} / D_{16}\right)^{1 / 2}
$$

where $D_{16}$ and $D_{84}$ are the diameters corresponding to the 16 th and 84 th mass percentile, respectively. It should be noted that all the PSD data is converted in terms of percentage of the total mass recovered at the different stages and IP (Induction port) of the ACI (Anderson Cascade Impactor) used during the experiment.

\subsubsection{Particle size distribution}

Aerosol size measurements, particles released by Spiriva Respimat inhaler, are compared under normal and humid conditions. Figure 5.24 shows the cumulative aerosol distribution (the value at the cut-off of $10 \mu \mathrm{m}$ represents the mass fraction of all particles below $10 \mu \mathrm{m}$ ) for both conditions with their associated standard deviation displayed as error bars. As can be seen, larger amount of fine particles with diameters less than $4.7 \mu \mathrm{m}$ (representing stages 3 to 7 in the ACI) are obtained at the lower relative humidity. This shift in particle distribution, which was also observed by Ziegler and Wachtel [99], and Martin and Finlay [100], is due to evaporation or condensation where the particles gain or lose mass from their surface [30]. It is conjectured here that the droplet size distribution measured at $90 \% \mathrm{RH}$ (relative humidity) closely resembles the distribution generated by the inhaler. For the 40-50\% RH conditions, generated inhaler droplets will experience a different evaporation rate, thus changing the measured size distribution. 


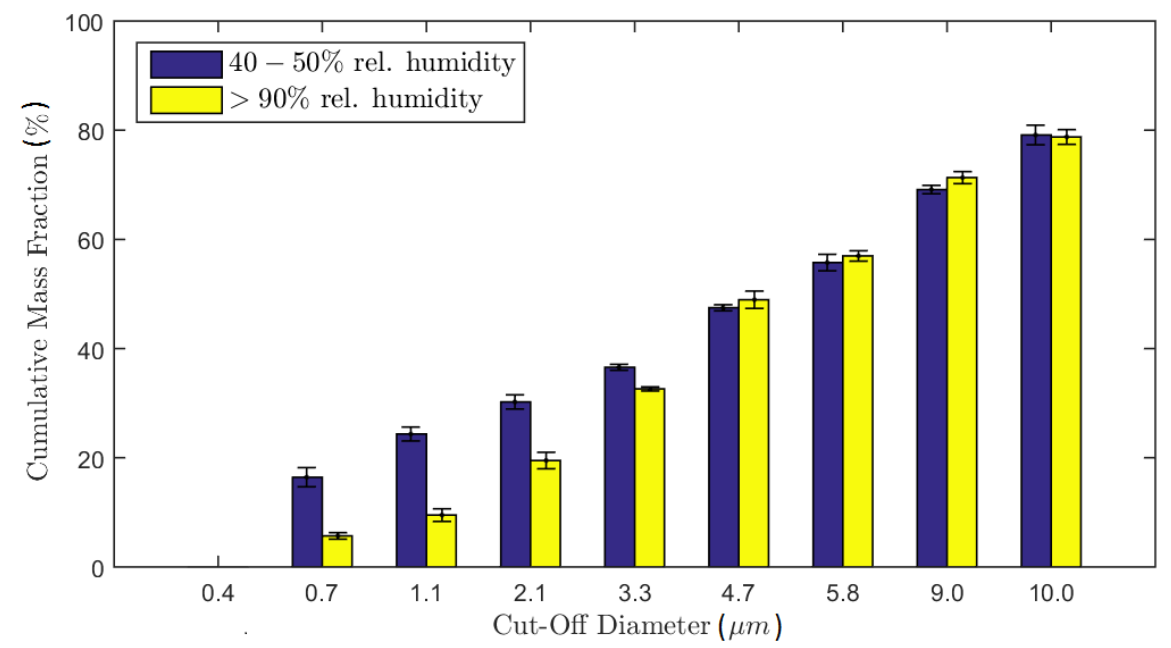

Figure 5.24: Aerodynamic particle size distribution under normal and humid conditions.

Table 5.7 summarizes the MMAD and GSD results for both normal and humid conditions, and their respective standard errors. Although there is a shift in the PSD due to the humidity effect, the difference in MMAD under normal $(5.0 \pm 0.5)$ and humid condition $(4.8 \pm 0.2)$ was negligible (4.0\%). In contrast, humidity had a greater impact on the GSD $(7.5 \pm 0.5$ under the normal condition compared to $2.8 \pm 0.2$ under the humid condition) resulting in a larger amount of fine particles, therefore, more drug is delivered to the lungs' alveoli.

Table 5.7: Particle size distributions of aerosol from Spiriva Respimat inhaler under normal and humid conditions.

\begin{tabular}{|c|c|c|c|}
\hline Condition & Relative Humidity $(\%)$ & MMAD $(\mu m)$ & GSD (-) \\
\hline Normal & $40-50$ & $5.0 \pm 0.5$ & $7.5 \pm 0.5$ \\
\hline Humid & $>90$ & $4.8 \pm 0.2$ & $2.8 \pm 0.2$ \\
\hline
\end{tabular}

Similarly, as can be observed in Figure 5.24, about $48 \%$ (48.9\% for $\mathrm{RH}>90 \%$ and $47.5 \%$ for $40-50 \% \mathrm{RH}$ ) of the medication delivered by the Respimat SMI deposited in the tracheo-bronchial and alveolar regions (aerosol with particle diameters between 
$0.4 \mu \mathrm{m}$ and $4.7 \mu \mathrm{m}$, ACI stages 3 to 7 ) which are sometimes referred to as the lung [101]. During the clinical study performed by Newman et al.,[102] on average $39.2 \%$ and $44.6 \%$ of fenoterol and flunisolide deposited in the lung, respectively. The slight difference in deposition obtained was mainly due to the steady inhalation rate (in addition to the usage of the induction port to simulate the medication losses on the actual mouththroat-trachea geometry of patients) used in the current study as compared to the inhalation technique described in Newman et al., [102] where the subjects inhaled slowly and deeply with a targeted rate of $30 \mathrm{~L} / \mathrm{min}$ and held their breath after inhalation for at least 10 seconds.

Figure 5.6 shows a comparison between both locations at $75 \mathrm{~mm}$ and $100 \mathrm{~mm}$ on the center line, downstream from the nozzle orifice using PDA. For both locations (at $75 \mathrm{~mm}$ and $100 \mathrm{~mm}$ ) only $8.0 \%$ and $17.0 \%$ of the medication delivered (aerosol with particle diameters less than $4.7 \mu \mathrm{m}$ ) by the Respimat SMI deposited in the tracheobronchial and alveolar regions, respectively. In addition, more medication is delivered cumulatively at the $100 \mathrm{~mm}$ location when compared to the previous one, where the difference is due to the evaporation process that affects the particles. The particle fraction measured using PDA is, however, smaller than the particle fraction measured via ACI as it was about $48 \%$. The reason for this is that the conditions of the experiment are different for each test. The PDA measurement is taken at the lab's ambient conditions where the humidity level is about $20 \%$, whereas, it is controlled for the ACI tests. Also, the PDA measurement is a point measurement and can differ from one location to another, while the ACI is a cumulative test for the whole dosage and for 20 actuations. It's been reported by Wachtel and Ziegler [47] that there was a significant change in aerosol size distribution in laser diffraction measurements of the Respimat spray as a function of relative humidity. Consequently, evaporation of the aerosol is anticipated to impact size distribution and deposition characteristics as the particles proceed downstream of the flow. 


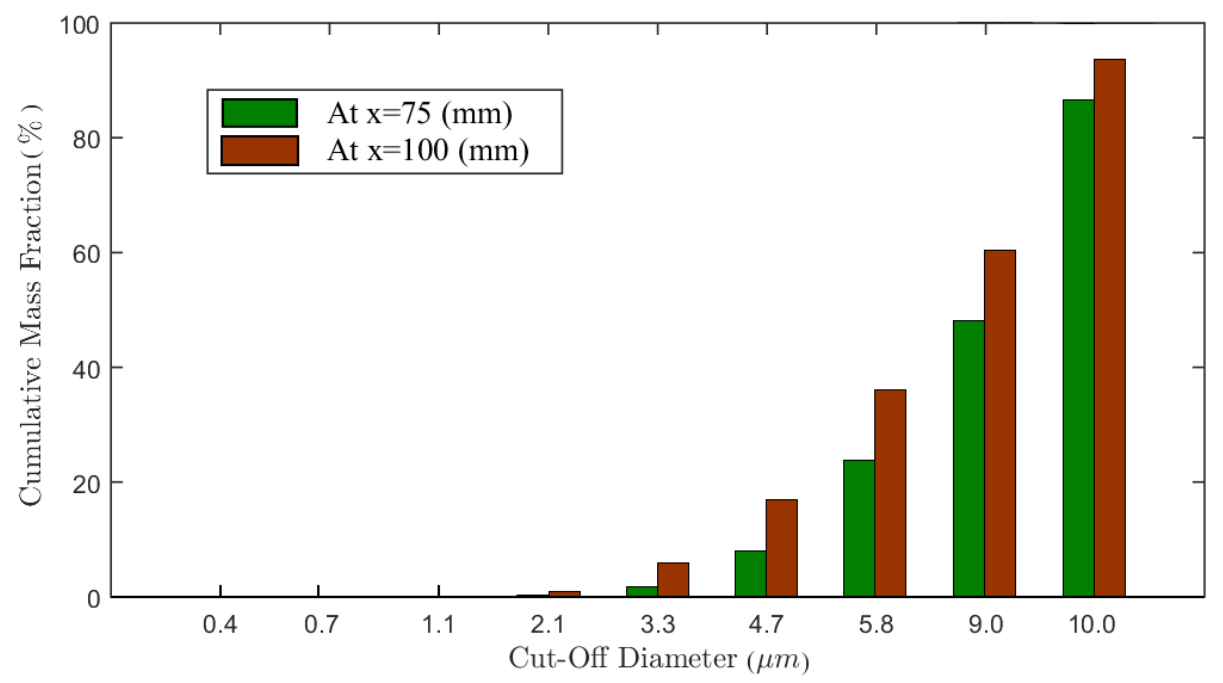

Figure 5.25: Aerodynamic particle size distribution for both locations $75 \mathrm{~mm}$ and 100 $m m$ using PDA.

\subsubsection{Aerosol deposition}

Figure 5.26 shows aerosol deposition measurements as percentage deposition of tiotropium bromide monohydrate at an inspirational flow rate of $28.3 \mathrm{~L} / \mathrm{min}$ for both humidity levels, at the IP and the ACI stages. That most of the deposition is in the IP for both humidity levels by about 21\%. Also, there is much difference in deposition through stages 0 to 3 for both conditions. However, the deposition in stages 4 and 5 at normal humidity $(40-50 \%)$ is relatively close about $6 \%$. Whereas at humid $(>90 \%)$ condition its $13 \%$ and $10 \%$ for both stages, respectively. Also, under normal conditions there is a greater percent deposition of medication in the stages 6 and 7 lung segments. The reason for the larger difference under humid conditions is due to the aerosols having larger mass and size. 


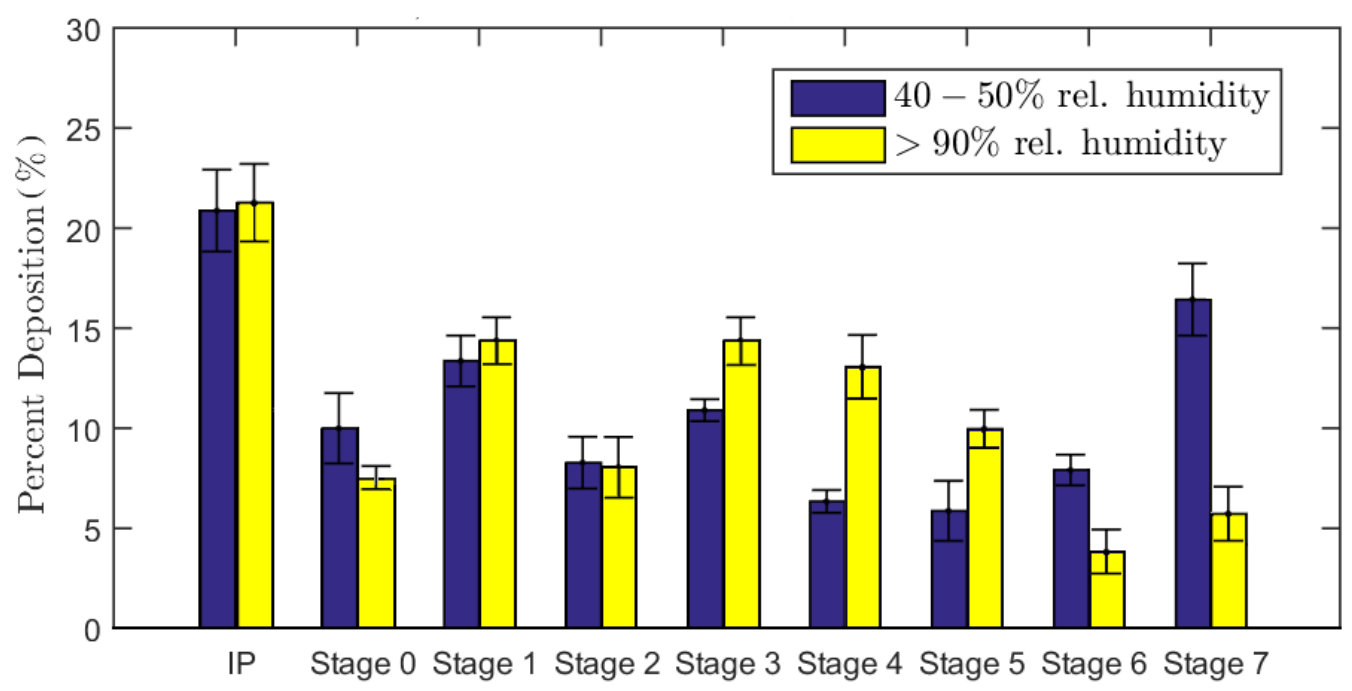

Figure 5.26: Percent deposition of medication at two relative humidity levels of (a) $40-50 \%$ and $(\mathrm{b})>90 \%$.

\subsubsection{Error and Uncertainty}

The accuracy of any measurement can not be entirely correct, therefore, to determine the quality of the measurement an uncertainty analysis is needed to indicate this feature in the measurement [103]. Measurement errors can occur from a variety of sources such as, data reduction errors, data acquisition and calibration [104]. Moreover, the total error is typically classified in two terms: a fixed (bias) error, and a random (precision) error [104].

The bias error is categorized as systematic or fixed, which also is counted as constant through the test. Hence, in repeated measurements of a certain group, each measurement has the same bias $[104,105]$. The total bias limit can be calculated by:

$$
b=\sqrt{\sum_{k=1}^{K} b_{k}^{2}}
$$

Where $K$ is the index for the bias and $b$ is the bias of the equipment. 
In quantitative experiments instrument bias is considered to be a source of measurement bias. From the apparatus used in PDA system measurement the bias errors have been specified as $2 \%$ for the BSA flow software, and $1 \%$ for the $2-\mathrm{D}$ traverse system. On the other hand, from the equipment used in the ACI experiments bias error been stated as $1.6 \%$ Agilent spectrophotometer, $0.7 \%$ for the flow meter, and for ACI each stage has its bias error which given in Table 5.8 [106].

Table 5.8: Bias error for ACI stages.

\begin{tabular}{|c|c|c|c|c|c|c|c|c|}
\hline Stage & 0 & 1 & 2 & 3 & 4 & 5 & 6 & 7 \\
\hline Bais error \% & 1.10 & 1.70 & 2.10 & 3.00 & 4.80 & 9.10 & 7.70 & 7.00 \\
\hline
\end{tabular}

The term precision is used to express the random measurement errors. Those errors can be caused by measuring instruments or environmental conditions. Also, they cannot be eliminated, but their effect can be reduced via incrementing sample sizes and performing several measurements. In order to obtain the precision error of the experiment, the standard deviation of the results needs to be calculated. In addition is required the student t-test value for a $95 \%$ confidence interval with five samples. In this case five samples were taken, giving a value of 2.132. The total error of the experiments is then obtained by combining both bias error and precision error, according to [104], using;

$$
U_{R S S}=\sqrt{\left(B^{2}+\left(t S_{\bar{x}}\right)\right)^{2}}
$$

Where $U_{R S S}$ is the uncertainty, $B$ is the bias, $t$ is the 95 th percentile point for the student t-test and $S_{\bar{x}}$ is the standard deviation. 


\subsubsection{Errors in measurements for PDA system}

The absolute measurement principle of the phase Doppler method requires no system calibration (Albrecht et al. [72]), however, this principle is unable to deliver an adequate measurement accuracy [107] . Also, since PDA measurements will detect information such as velocity and size of any particle that passes through the measurement volume, extra particles that pass through the measurement volume in a given time frame, will result in a bias in the data. This is particularly prevalent in high speed flows. Consequently, averaging the data would over-estimate the acquired mean, and an error would also be introduced in the estimated variance. To solve this issue, by weighing each data sample through the amount of time frame it takes until the next particle arrives. This approach to evaluate the uncertainty of the droplet information is suggested by Kapulla et al. [107]. The scatter of the velocity and diameter estimate at a single point can be treated as normally distributed. Therefore, the determination of the mean of both velocity or diameter uncertainty can be achieved by initially determining if each measured droplet is statistically independent. A lag time for typical atomization sprays is commonly taken between 2 and $4 \mathrm{~ms}$, thus, resulting in a statistically independent acquisition frequency of $250<f<500 \mathrm{~Hz}[107]$.

The mean square error, or the estimator of the variance $\sigma_{\bar{d}}^{2}$, of the calculated count mean diameter $\bar{d}$, as shown in Equation 5.7

$$
\bar{d}=\frac{1}{N} \sum d_{i}
$$

can be calculated by means of

$$
\sigma_{\bar{d}}^{2}=\frac{\sigma_{d}^{2}}{N}
$$

with the estimator of the variance $\sigma_{d}^{2}$ of the observations the measured diameters $d_{i}$ $[72]$ 


$$
\sigma_{\bar{d}}^{2}=\frac{1}{N} \sum\left(d_{i}-\bar{d}\right)^{2}
$$

Therefore, by assuming a normal distribution for the scatter of the estimates for $\bar{d}$, the probability of being within $\pm 1.96 \sigma_{\bar{d}}$ of the true value would be $95 \%$. Table 5.9 shows the mean particle diameter measurement with standard deviation $\sigma_{\bar{d}}$ for the four tested inhalers along with the percentage of the independent samples using the above criteria. This analysis resulted in average uncertainty value of $\pm 0.0224 \mu \mathrm{m}$ to $\pm 0.0268 \mu \mathrm{m}$ on the of mean particle diameter for the $0(\mathrm{~mm})$ location. It also, shows that $37.78 \%$ to $90.96 \%$ of particles acquired are statistically independent at the same location for all tested inhalers. The average uncertainty values increase as the distance from the mouthpiece edge becomes greater for all inhalers except for Ventolin HFA at the location $100(\mathrm{~mm})$ where it is only $\pm 0.0260 \mu \mathrm{m}$. The tables demonstrate these cases and can be found in Appendix A.

Table 5.9: Measurement errors for particle size using PDA system for tested inhalers at $x=0(\mathrm{~mm})$ at $95 \%$ level of confidence.

\begin{tabular}{|l|c|c|c|c|c|}
\hline Inhaler type & $\begin{array}{c}\text { Independent } \\
\text { samples } \%\end{array}$ & $\begin{array}{c}\text { SD } \\
\text { value } \sigma_{\bar{d}} \\
(\mu m)\end{array}$ & $\begin{array}{c}\text { Bias } \\
\text { limit } \\
(\mu m)\end{array}$ & $\begin{array}{c}\text { Precision } \\
\text { limit } \\
(\mu m)\end{array}$ & $\begin{array}{c}\text { Uncertainty } \\
( \pm \mu m)\end{array}$ \\
\hline Apo-Salvent CFC Free & 42.08 & 0.05 & 0.02 & 0.01 & 0.02 \\
\hline Airomir HFA & 37.78 & 0.05 & 0.02 & 0.01 & 0.02 \\
\hline Novo-Salbutamol HFA & 41.93 & 0.05 & 0.02 & 0.01 & 0.02 \\
\hline Ventolin HFA & 42.23 & 0.05 & 0.02 & 0.01 & 0.02 \\
\hline Spiriva Respimat & 90.96 & 0.010 & 0.02 & 0.00 & 0.02 \\
\hline
\end{tabular}

Table 5.10 shows the mean particle velocity measured with uncertainty value at $x=0 \mathrm{~mm}$, away from the origin along the centerline. Ventolin HFA presents a high 
average uncertainty value for the mean particle velocity of $\pm 0.8447 \mathrm{~m} / \mathrm{s}$, whereas the Spiriva Respimat produced the lowest average uncertainty value of $\pm 0.0228 \mathrm{~m} / \mathrm{s}$. The average uncertainty is the lowest at the second location, however it is slightly higher at the third location, with the exception of Ventolin HFA which has the same value of $\pm 0.1333 \mathrm{~m} / \mathrm{s}$ at both $x=75 \mathrm{~mm}$ and $x=100 \mathrm{~mm}$ locations. The uncertainty analysis results on the mean particle velocity for these two locations can be found in Appendix A.

Table 5.10: Measurement errors for particle velocity using PDA system for tested inhalers at $x=0(\mathrm{~mm})$ at $95 \%$ level of confidence.

\begin{tabular}{|l|c|c|c|c|c|}
\hline Inhaler type & $\begin{array}{c}\text { Independent } \\
\text { samples } \%\end{array}$ & $\begin{array}{c}\text { SD } \\
\text { value } \\
\sigma_{\bar{d}}(\mathrm{~m} / \mathrm{s})\end{array}$ & $\begin{array}{c}\text { Bias } \\
\text { limit } \\
(\mathrm{m} / \mathrm{s})\end{array}$ & $\begin{array}{c}\text { Precision } \\
\text { limit } \\
(\mathrm{m} / \mathrm{s})\end{array}$ & $\begin{array}{c}\text { Uncertainty } \\
( \pm \mathrm{m} / \mathrm{s})\end{array}$ \\
\hline Apo-Salvent CFC Free & 42.08 & 0.25 & 0.02 & 0.29 & 0.29 \\
\hline Airomir HFA & 37.78 & 0.30 & 0.02 & 0.43 & 0.43 \\
\hline Novo-Salbutamol HFA & 41.93 & 0.17 & 0.02 & 0.43 & 0.14 \\
\hline Ventolin HFA & 42.23 & 0.43 & 0.02 & 0.84 & 0.84 \\
\hline Spiriva Respimat & 76.29 & 0.031 & 0.02 & 0.00 & 0.02 \\
\hline
\end{tabular}

Table 5.11 summarize the measurement errors for particle size and velocity using PDA system for tested inhalers at $x=0(\mathrm{~mm})$.

\subsubsection{Errors in measurements for particle size and deposition using ACI}

Using the above methods of calculation, the following errors in measurement were found for the experiments using ACI system under normal humidity (40-50\% RH ) for both the particle size and aerosol deposition, which can be seen in Tables 5.12 and 5.13 respectively. Full error measurements for the experiments using ACI system under 
Table 5.11: Measurement errors for particle size and velocity using PDA system for tested inhalers at $x=0(\mathrm{~mm})$ at $95 \%$ level of confidence.

\begin{tabular}{|l|c|c|}
\hline Inhaler type & Particle size $D_{10}(\mu \mathrm{m})$ & Particle velocity $(\mathrm{m} / \mathrm{s})$ \\
\hline Apo-Salvent CFC Free & $2.83 \pm 0.02$ & $32.87 \pm 0.29$ \\
\hline Airomir HFA & $2.55 \pm 0.02$ & $32.40 \pm 0.43$ \\
\hline Novo-Salbutamol HFA & $2.78 \pm 0.02$ & $26.90 \pm 0.14$ \\
\hline Ventolin HFA & $3.75 \pm 0.02$ & $43.20 \pm 0.84$ \\
\hline Spiriva Respimat & $3.58 \pm 0.02$ & $4.52 \pm 0.02$ \\
\hline
\end{tabular}

normal humidity ( $>90 \% \mathrm{RH})$ are given in Appendix A.

\subsection{High Speed Camera results}

\subsubsection{Spray Distance and Duration for Spiriva Respimat inhaler}

The average distance travelled by the spray as a function of time was extracted and compared with a similar experiment from Hochrainer et al. [49]. The result (Figure 5.27 ) shows similar trend within the first $0.1 \mathrm{~s}$. It was also found that the spray travelled a total of $250-270 \mathrm{~mm}$ in about $1.5 \mathrm{~s}$.

\subsubsection{Respimat SMI versus pMDI}

The spray characteristics of Spiriva Respimat SMI and Ventolin HFA pMDI were compared using the same experimental setup. Both videos recorded at $500 \mathrm{fps}$ for $2 \mathrm{~s}$. As previously mentioned, the Respimat SMI travelled a total of $250-270 \mathrm{~mm}$ in about 1.5 s. On the other hand, the spray for the pMDI travelled about $270-300 \mathrm{~mm}$ in about 
Table 5.12: Measurement errors for particle size using ACI system under normal humidity (40-50\% RH ) at $95 \%$ level of confidence.

\begin{tabular}{|l|c|c|c|}
\hline Stage & $\begin{array}{c}\text { Bias } \\
\text { limit }(\mu m)\end{array}$ & $\begin{array}{c}\text { Precision } \\
\text { limit }(\mu m)\end{array}$ & $\begin{array}{c}\text { Uncertainty } \\
( \pm \mu m)\end{array}$ \\
\hline 0 & 0.02 & 0.00 & 0.02 \\
\hline 1 & 0.02 & 0.00 & 0.02 \\
\hline 2 & 0.02 & 0.00 & 0.02 \\
\hline 3 & 0.03 & 0.00 & 0.03 \\
\hline 4 & 0.05 & 0.00 & 0.05 \\
\hline 5 & 0.09 & 0.00 & 0.09 \\
\hline 6 & 0.07 & 0.00 & 0.07 \\
\hline 7 & 0.07 & 0.00 & 0.07 \\
\hline
\end{tabular}

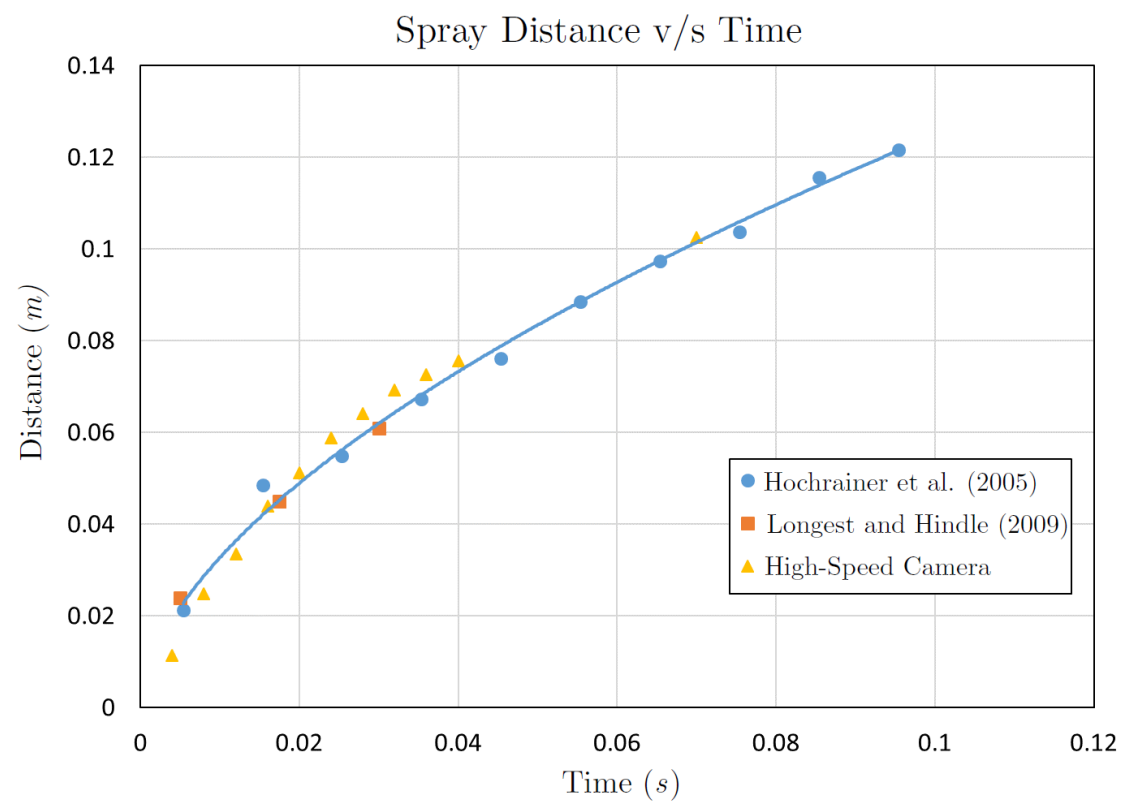

Figure 5.27: Distance travelled by the spray as a function of time. 
Table 5.13: Measurement errors for deposition on ACI system under normal humidity (40-50\% RH ) at $95 \%$ level of confidence .

\begin{tabular}{|l|c|c|c|}
\hline Stage & Bias & $\begin{array}{c}\text { Precision } \\
\text { limit } \%\end{array}$ & $\begin{array}{c}\text { Uncertainty } \\
\%\end{array}$ \\
\hline IP & 0.01 & 0.00 & 0.01 \\
\hline 0 & 0.02 & 0.00 & 0.02 \\
\hline 1 & 0.02 & 0.00 & 0.02 \\
\hline 2 & 0.02 & 0.00 & 0.02 \\
\hline 3 & 0.03 & 0.00 & 0.03 \\
\hline 4 & 0.05 & 0.00 & 0.05 \\
\hline 5 & 0.09 & 0.00 & 0.09 \\
\hline 6 & 0.07 & 0.00 & 0.07 \\
\hline 7 & 0.07 & 0.00 & 0.07 \\
\hline
\end{tabular}


$0.3 \mathrm{~s}$. This considerably longer spray duration was found to help deliver the medication more efficiently. Figure 5.28(a) shows that at the beginning of the actuation Spiriva Respimat spray has a high density than Ventolin HFA. In Figures 5.28(b) and (c) Spiriva Respimat spray after a $100 \mathrm{~mm}$ travel distance the spray deflects down the horizontal line, where as Ventolin HFA goes slightly upwards. Then, Spiriva Respimat spray keeps fluctuating until the end of the spray time Figures 5.28(e) and (f).

\section{$5.4 \quad$ Numerical results}

Transient simulations is performed for the numerical analysis to find how well the software package can predict the pMDI behavior and velocity distribution. Then, the results are validated against the detailed experimental data obtained before.

The simulations results are described using images, which were obtained from the results' view mode available in ANSYS CFX. Using particle tracking and URANS, it was possible to extract the data of the particles for multiple time steps of the simulation showing the time evolution of the process.

The contours of velocity along a longitudinal mid-section plane and tracked particles along the fluid domain at various time steps are shown in Figures 5.29 and 5.30, respectively. As illustrated in Figure 5.29 it is possible to simulate the deflecting flow of the pMDI spray (the deflection in the flow is a result of implementing equation 4.1 at the inlet), based on the findings of Crosland [7]. The velocity magnitude of air in the domain at the time of $t=0.0015 \mathrm{~s}$ as well as in $t=0.03 \mathrm{~s}$, show the deflection in the spray. Figure $5.29(\mathrm{f})$ at $t=0.182 \mathrm{~s}$, corresponds to the end of the injection of the medication from the pMDI.

A direct analysis of the particles diameter and positioning are given for the particles as spheres scaled by their diameter are illustrated in Figure 5.30. Also, small particles 


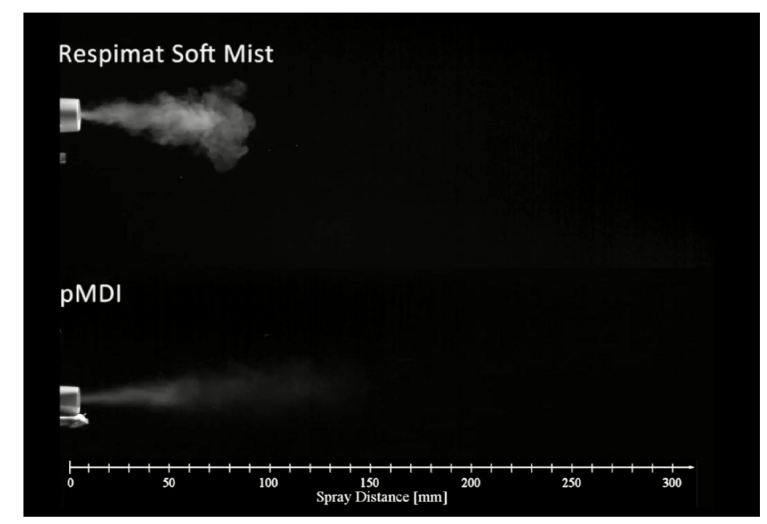

(a) At $t=0.0607 s$.

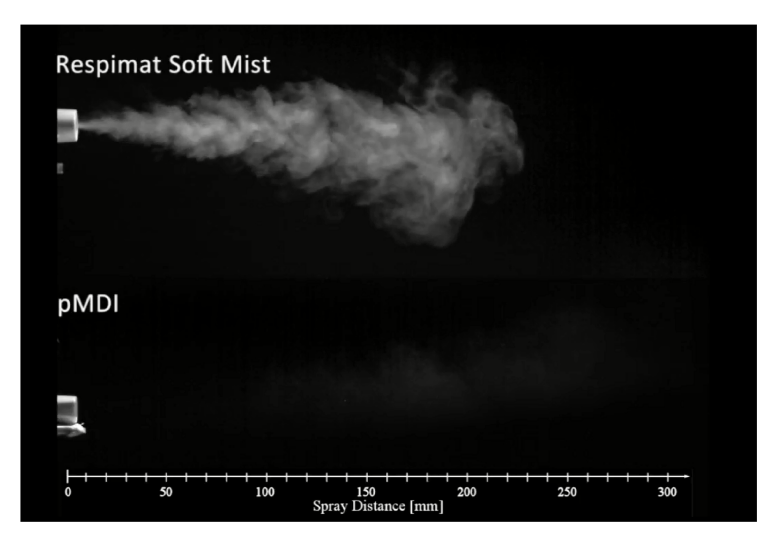

(c) At $t=0.283 \mathrm{~s}$.

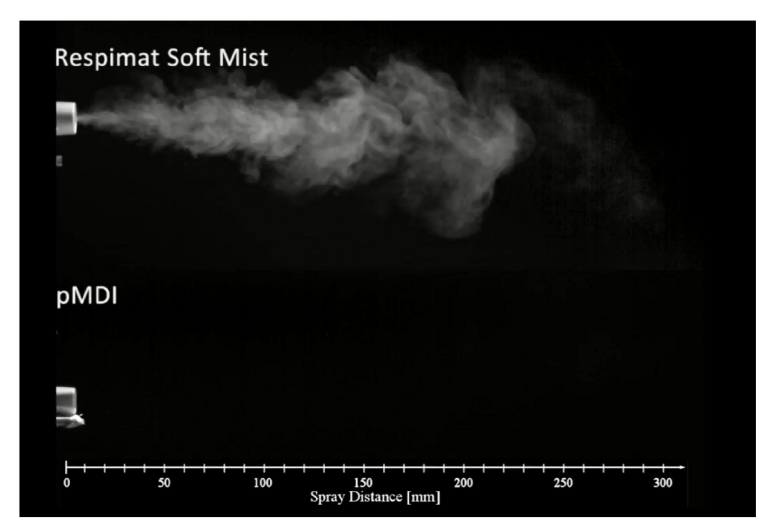

(e) At $t=1.41 \mathrm{~s}$.

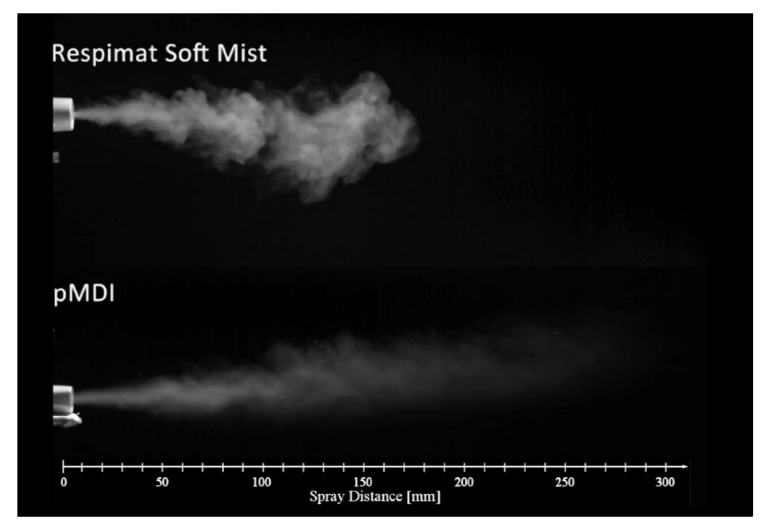

(b) At $t=0.160 \mathrm{~s}$.

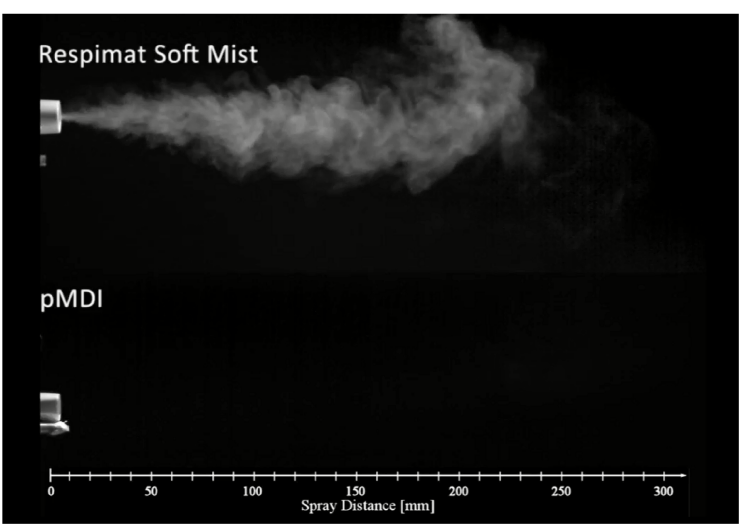

(d) At $t=0.816 \mathrm{~s}$.

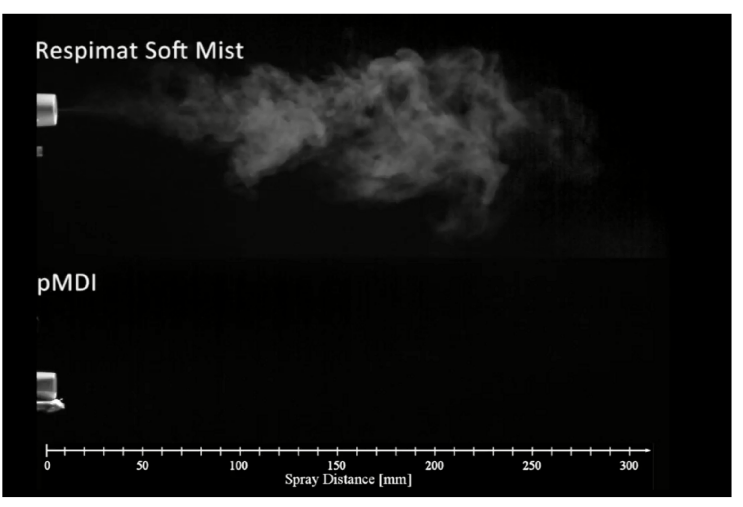

(f) At $t=1.47 \mathrm{~s}$.

Figure 5.28: Spray characteristics of Respimat SMI versus Ventolin HFA pMDI. 


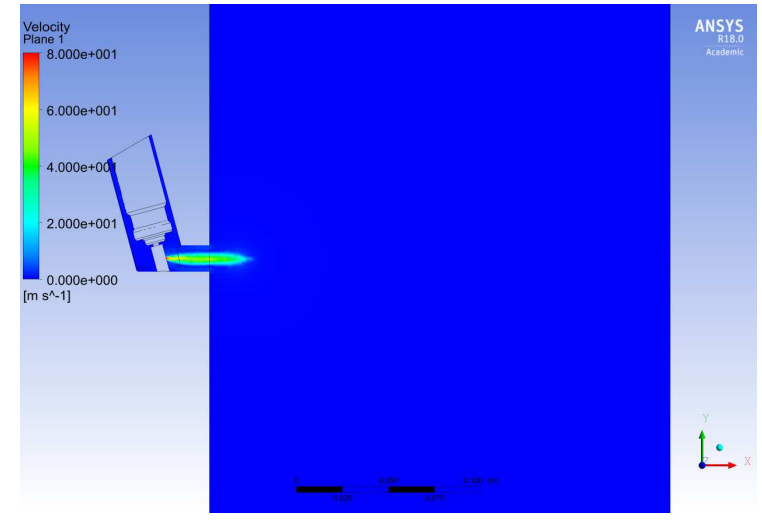

(a) $\mathrm{t}=0.001 s$.

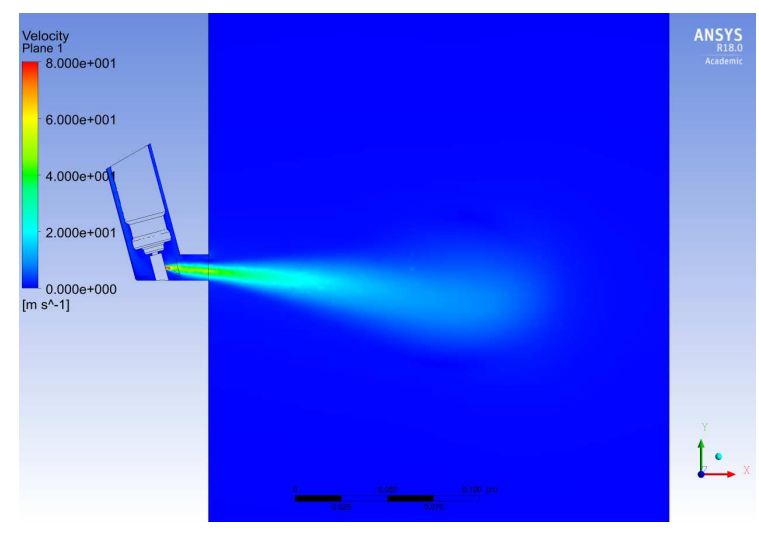

(c) $\mathrm{t}=0.03 \mathrm{~s}$.

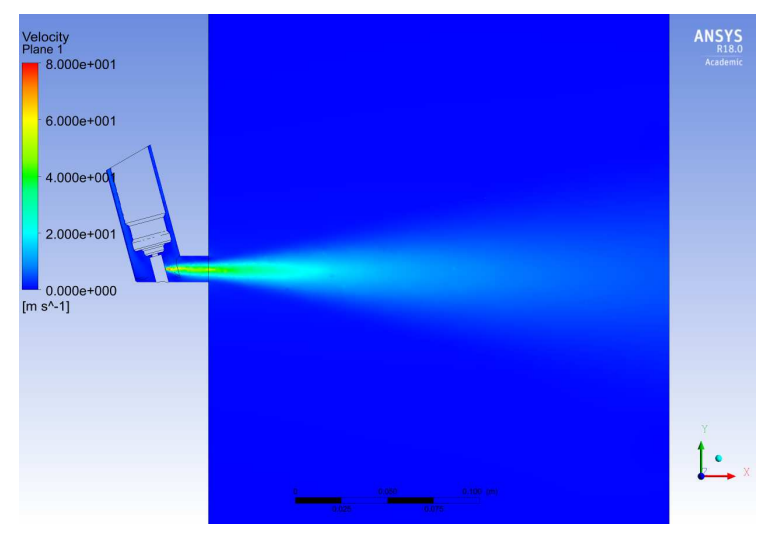

(e) $\mathrm{t}=0.18 \mathrm{~s}$.

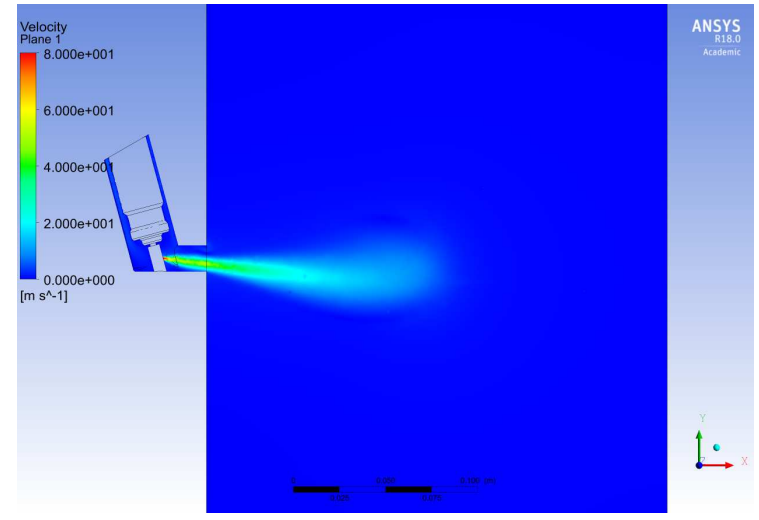

(b) $\mathrm{t}=0.0015 \mathrm{~s}$.

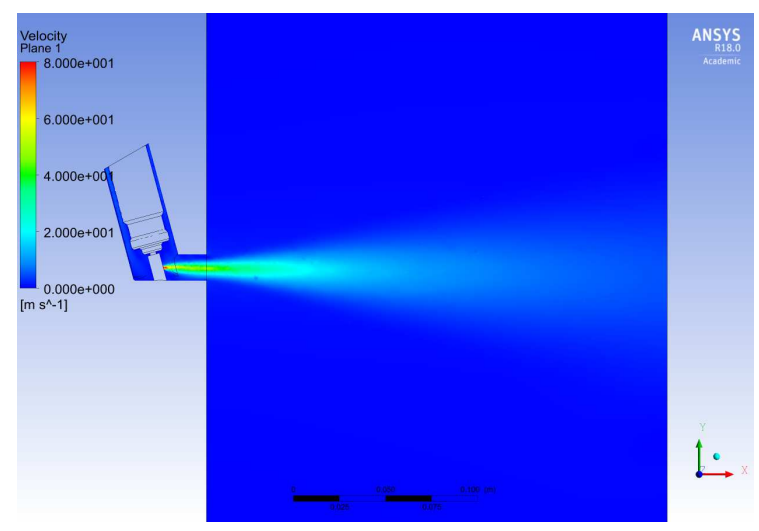

(d) $\mathrm{t}=0.14 \mathrm{~s}$.

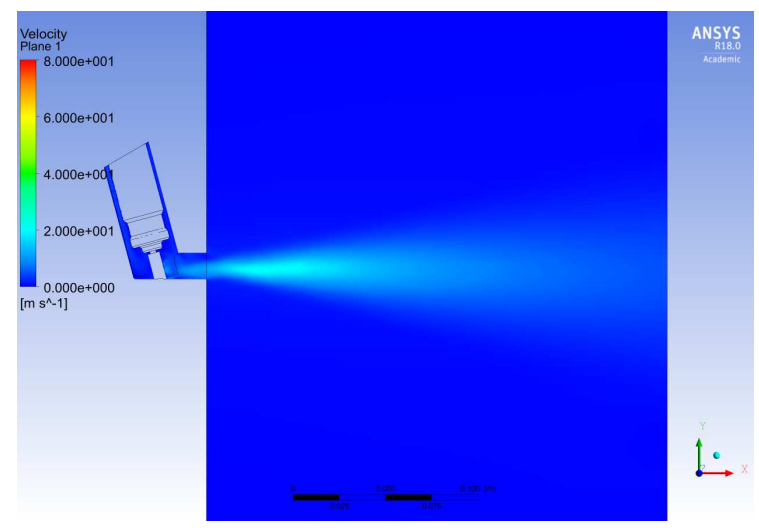

(f) $\mathrm{t}=0.182 \mathrm{~s}$.

Figure 5.29: Velocity magnitude contours of the air in the domain. 
are close to the mouthpiece zone while the larger ones are at the end of the spray. The used Rosin-Rammler particle size distribution produces a result in the anticipated manner, however, the CFX solver injects the same number of particle streams at each instant of the injection, this way of injection could impact the shape of the spray.

Figure 5.31 illustrates the comparison between the spray velocity simulation against the experimental data at three axial locations ( $\mathrm{x}=0,75$, and $100 \mathrm{~mm}$ ). Relatively good agreement is obtained at 0 and $75 \mathrm{~mm}$ locations, although discrepancies exist for 100 $m m$ velocity distributions. 


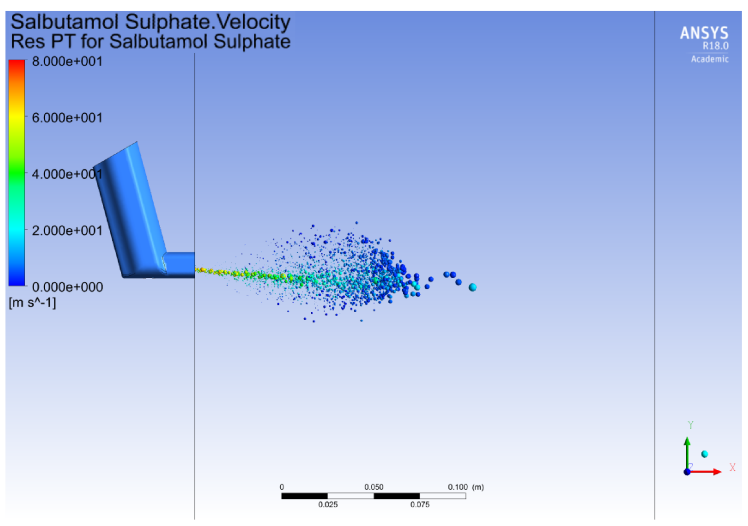

(a) $\mathrm{t}=0.01 s$.

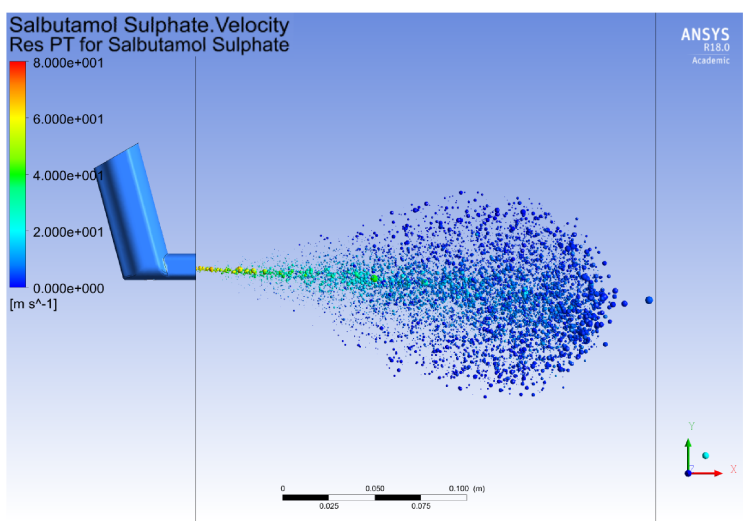

(c) $\mathrm{t}=0.04 \mathrm{~s}$.

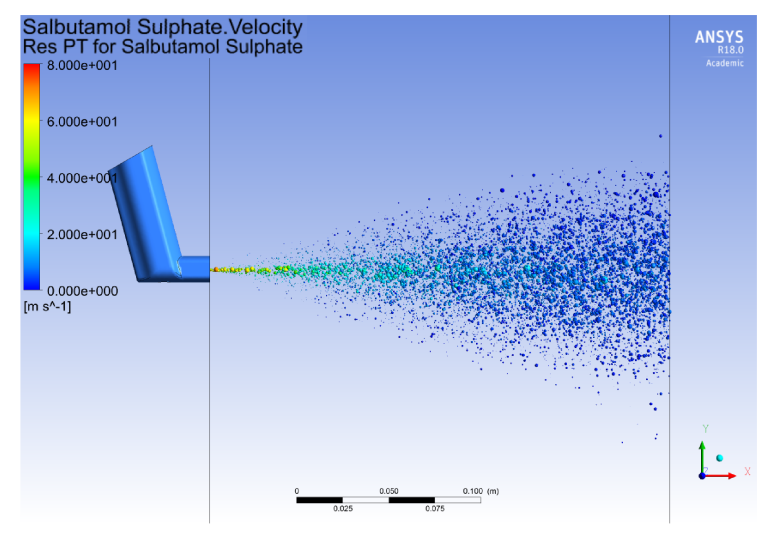

(e) $\mathrm{t}=0.14 \mathrm{~s}$.

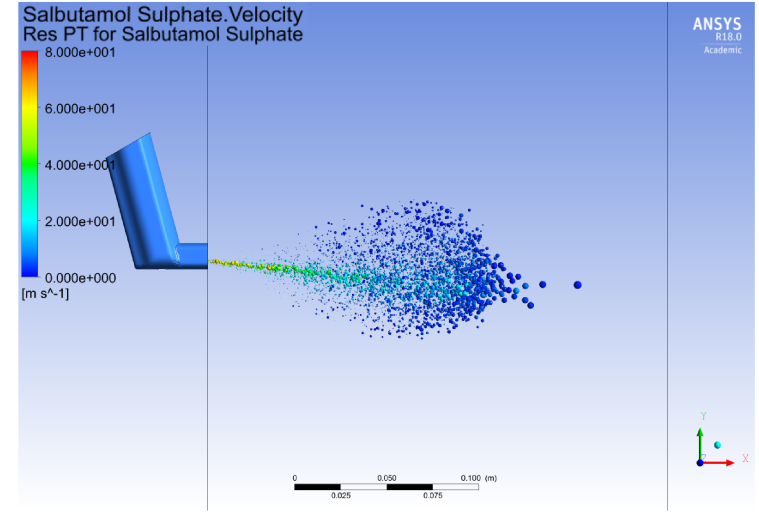

(b) $\mathrm{t}=0.02 s$.

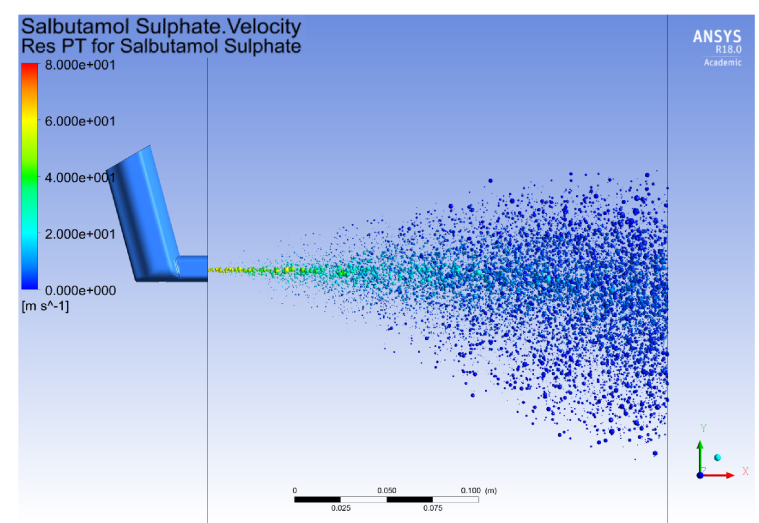

(d) $\mathrm{t}=0.08 s$.

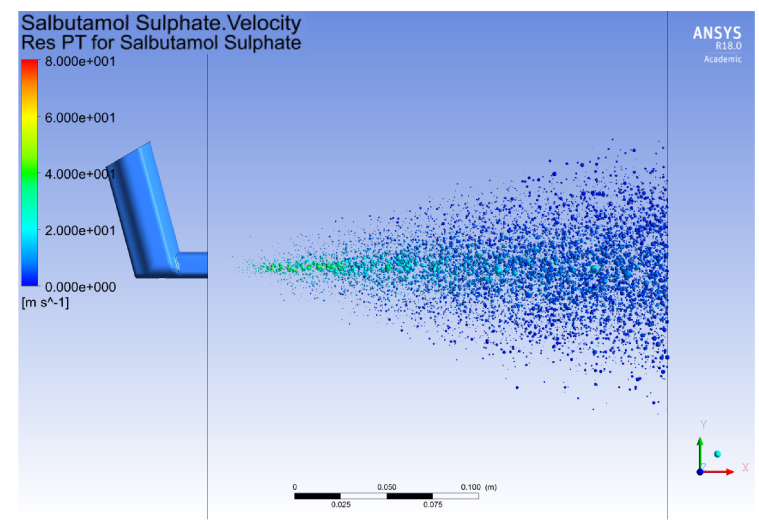

(f) $\mathrm{t}=0.182 \mathrm{~s}$.

Figure 5.30: Velocity magnitude contours of the particle in the domain. 


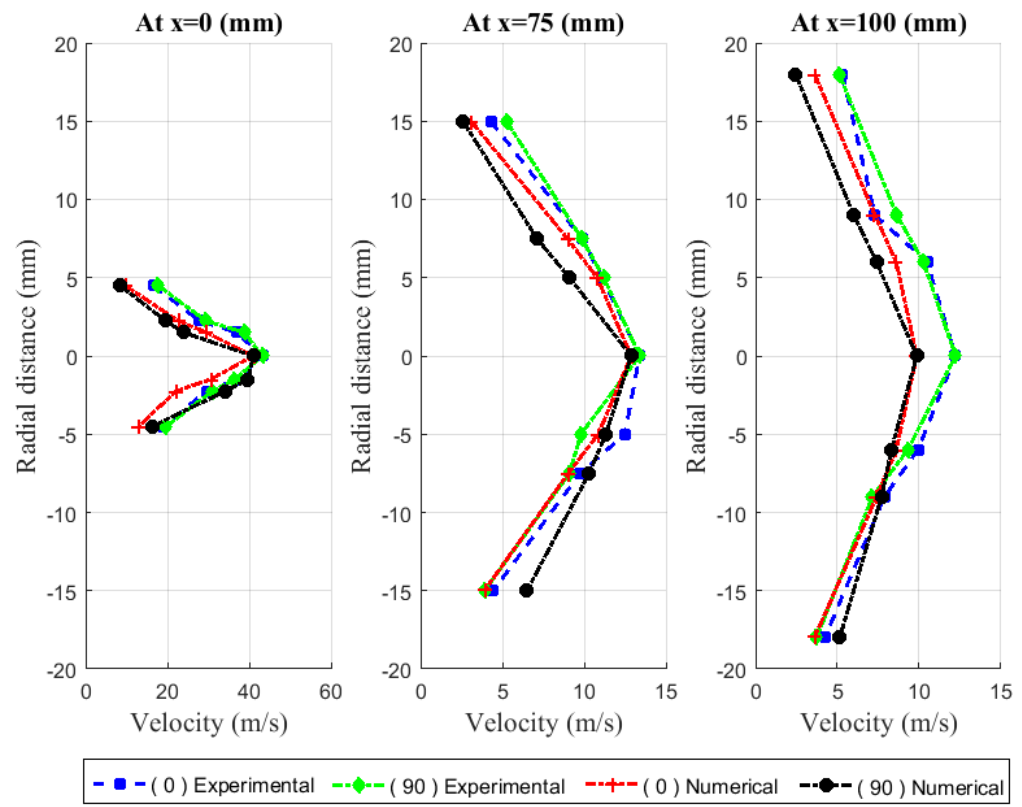

Figure 5.31: Spray velocity comparison numerical against experimental. 


\section{Chapter 6}

\section{Conclusions and Recommendations}

\subsection{Summary of results}

This research investigated both particle size and velocity for four commercial pMDIs and one SMI which were characterized using PDA. The investigation was carried out to examine the effect of sampling distance on the particle velocity and particle size. The following was found: firstly, for temporal analysis of experimental results for the inhalers spray, for all tested pMDI brands, the spray velocities were bimodal in time, with two velocity peaks; the first one occurring as the spray was leaving the mouthpiece and the second around 60,95, 95, and $115 \mathrm{~ms}$ later for the tested inhalers, with a drop in the velocity occurring in between the two peaks. The transient event duration for the four inhalers is different for each of them, where it is about $180 \mathrm{~ms}$ for the Ventolin HFA. Whereas the transient event duration for Novo-Salbutamol HFA, Airomir HFA, and Apo-Salvent CFC Free is 290, 220, and $220 \mathrm{~ms}$, respectively. In contrast, the spray time for the Spiriva Respimat SMI is much longer when compared to pMDI inhalers, as it lasts for approximately $1500 \mathrm{~ms}$. In addition, the velocity contour results show that the spray characteristics demonstrate a slight asymmetrical behavior with high velocity was observed in the radial distance $R / 3$ region. It also demonstrates the 
phenomenon of two peaks throughout the interval times for the tested pMDIs. For particle diameters temporal analysis for all tested pMDIs, that the average diameter of the released particles is below the mean at the start of the actuation, when the spray density is high, then the average increases gradually from less than the mean $D_{10}$ to higher than that with fluctuations until it reaches about $100 \mathrm{~ms}$. Following this, the average decreases with fluctuations below the mean until the end of the spray duration.

Secondly, for spatial temporal analysis of experimental results for the inhaler sprays, there is a difference in the mean particle velocities at the centerline for the tested inhalers; at the sampling point $0 \mathrm{~mm}$, it ranged from 26.94 to $43.20 \mathrm{~m} / \mathrm{s}$. Whereas it was 5.49 to $12.22 \mathrm{~m} / \mathrm{s}$, at $100 \mathrm{~mm}$ away from the origin. The Ventolin HFA pMDI demonstrated the highest particle velocity at all three locations. The Apo-Salvent CFC Free pMDI shows the greatest decrease in particle velocity at both locations 75 and $100 \mathrm{~mm}$. A comparison of particle velocity at both locations 0 and $75 \mathrm{~mm}$ showed that the decrease in particle velocity in Apo-Salvent CFC Free is higher than the other products, with $75.99 \%$. This decrease is relatively close to Airomir HFA with $73.27 \%$. While for Ventolin HFA and Novo-Salbutamol HFA, the decrease in particle velocity was $69.00 \%$ and $72.86 \%$, respectively. The decline in velocity between the second and third location was not significant in comparison to any of the brands. Moreover, the results of the spatial analysis in cylindrical-polar coordinates produced an asymmetrical profile almost all inhalers. The mean velocity spatial analysis for Spiriva Respimat inhaler decreases from $10.95 \mathrm{~m} / \mathrm{s}$ at the edge of the mouthpiece to $1.33 \mathrm{~m} / \mathrm{s}$ at the $100 \mathrm{~mm}$ location. It has a drop in the velocity between point 0 and both points 75 and $100 \mathrm{~mm}$ that both locations have an equal percentage in velocity drop with $70.13 \%$ and 70.57 $\%$, respectively.

Particle diameters are statistically different for each brand of tested inhalers at all locations except for Apo-Salvent CFC Free and Novo-Salbutamol HFA at $0 \mathrm{~mm}$ location. $D_{10}$ at $0 \mathrm{~mm}$ station, the values varied for all pMDIs, ranging from $2.55 \mu \mathrm{m}$ for 
Airomir HFA to $3.75 \mu \mathrm{m}$ for the Ventolin HFA, whereas at the second station $(75 \mathrm{~mm})$, it is relatively close for all brands, except the Ventolin HFA, which had the smallest diameter with $1.84 \mu \mathrm{m}$. Also, the MMD $\left(D_{V 50}\right)$ has the smallest diameter compared to $D_{10}$ and $D_{30}$, where it ranged from $1.26 \mu \mathrm{m}$ to $3.20 \mu \mathrm{m}$ at $0 \mathrm{~mm}$ location for the pMDI, where the Ventolin HFA has the highest and Airomir HFA the lowest, respectively. For the Spiriva Respimat SMI, $D_{10}$ values varied for all locations, ranging from $3.97 \mu \mathrm{m}$ at the point $-18.5 \mathrm{~mm}$ to $3.67 \mu \mathrm{m}$ at $100 \mathrm{~mm}$ location. Whereas, $D_{30}$ and $D_{V 50}$ for Spiriva Respimat inhaler show higher values in comparison to pMDI inhalers for all three locations. The size distribution data were used as the source data to calculate three probability distribution functions, the Rosin-Rammler PDF demonstrates better curve fitting when compared against the other two PDFs (lognormal and Nakagami) for tested pMDI inhalers. On the other hand, log-normal PDF shows the best curve fitting for Spiriva Respimat SMI at both locations -18.5 and $0 \mathrm{~mm}$.

Thirdly, the current study shows no change in the Respimat SMI particle size distribution in a humid environment (>90\% relative humidity, $\mathrm{RH})$ when compared against normal conditions (indoors, air-conditioned environment, 40 to $50 \% \mathrm{RH}$ ), that the difference in MMAD under normal $(5.0 \pm 0.5)$ and humid condition $(4.8 \pm 0.2)$ was negligible $(4.0 \%)$. In contrast, humidity had a greater impact on the GSD $(7.5 \pm 0.5$ under the normal condition compared to $2.8 \pm 0.2$ under the humid condition) resulting in a larger amount of fine particles, therefore, more drug is delivered to the lungs' alveoli. Nonetheless, the prediction of inhaled medication is about $48 \%$ gives better delivery to the lungs, which is still quite significant when compared against common pMDI. That pMDIs deliver between $8-20 \%$ of the metered dose to the lungs $[21,108]$.

Finally, numerical simulations were performed utilizing the experimental data, using ANSYS CFX software package to solve the unsteady Reynolds averaged Navier Stokes equations and its abilities in Lagrangian particle tracking and shear stress transport turbulence models to predict the pMDI spray behaviour. It was found that the results 
showing the contours of velocity along a longitudinal mid-section plane and as streams of tracked particles along the fluid domain and particle tracking at various time steps that it was possible to simulate the flow of the pMDI spray. In addition, the compaction between the experimental and the numerical velocity on both axis $0^{\circ}$ and $90^{\circ}$ reveled that the numerical velocity at both locations 0 and $75 \mathrm{~mm}$ is in good agreement with the experimental one. However, the numerical velocity at locations $100 \mathrm{~mm}$ is lower than the experimental one on both $0^{\circ}$ and $90^{\circ}$ axes.

\subsection{Summary of contributions}

This work provides important contributions to the understanding of the pMDIs and the Respimat SMI spray behaviour. The major accomplishments follow:

1. Characterization of Spray Velocities from a Pressurized Metered-Dose Inhaler. Poster presented at American Association for Aerosol Research (AAAR) 2013 Annual Conference.

2. Characterization of Medication Velocity and Size Distribution from Pressurized Metered-Dose Inhalers by Phase Doppler Anemometry. The results showed the deflection in the spray in several locations which can be considered the first study in the field. Also, this shows why most of the patients complain about the medication taste in their mouth after the inhalation of their medication. This work was published in the Journal of Aerosol Medicine and Pulmonary Drug Delivery in 2016 [109].

3. The results obtained for aerosol deposition measurements with ACI, they fill a gap 
in the literature, providing a better understanding of medication delivery to the lungs. The results indicate that the inhaled medication is delivered to the lungs, which is still quite significant when compared to common pressurized metered dose inhalers. This work will appear in Journal of Respiratory Therapy in 2018.

4. The results obtained for Spiriva Respimat SMI for both method PDA and CCD High Speed Camera deliver new information not found in the literature. As it provides better understanding of the spray behaviour and the increase in the particle diameter at the end of the spray duration. Also, these results will be important for researchers who are investigating particle deposition in the lungs and to those seeking to validate numerical results.

5. The data implementation in the CFD software verified experimentally (predicting experiments performed with the PDA), constitutes another contribution, ensuring the proper implementation of the experimental data and the proper capability of ANSYS CFX software to predict the pMDI spray behaviour in both single phase (air) and particulate domains. This work provides a framework for simulation of spray flows of different inhalers as well as confirming that the equation given by Matida and Alhegagi [84] gives a reasonable deflection angle of the spray velocity. As it was clear that the results exhibit relatively good agreement between simulation and experiment.

\subsection{Recommendations for future work}

There are a number of ways that this research could be developed further, in both experimental and in numerical sides. The following are recommendations for improving 
the experimental results of this thesis:

1. Different pMDI inhalers (as some have different nozzle design and dimensions) with different medication and propellants could also be assessed in the same way.

2. In this study the pMDI inhalers and the Spiriva Respimat SMI are actuated in an open air when PDA is used. To simulate the patient inhalation and to investigate the effect of the co-flow on the spray, an air flow is to be forced through the pMDI casing and through Spiriva Respimat SMI' vents at different flow rates to simulate a patient inhalation.

3. Add-on devices (spacers) can be attached to the pMDI inhaler and the Spiriva Respimat SMI in order to characterize the flow inside the spacer, which could be accomplished by providing an air flow via a vacuum pump.

In order to improve the numerical results through the CFD simulations the following concepts could be implemented:

1. In a real-life case the patient's already taking breath prior to the device actuation. Therefore, the domain could be initialised or by providing a very low flow rate through the pMDI casing during the simulation.

2. To achieve better and accurate results to assess the behaviour of the flow, Large Eddy Simulation (LES) could be used to simulate the eddies in the turbulent flow emitted from the inhaler.

As a further improvement, thereafter, a novel design of nozzle of the actuator with the 
help of PIV technique, would eliminate or reduce the deflection in the velocity spray of the pMDI inhalers. 


\section{Bibliography}

[1] P. H. A. of Canada, "Life and breath: respiratory disease in canada," 2007.

[2] P. H. A. of Canada, "Fast facts about asthma: data compiled from the 2011 survey on living with chronic diseases in canada," 2011.

[3] C. H. M. Survey, "Chronic obstructive pulmonary disease under-diagnosed in canadian adults: Results from cycles 3 and 4 (2012 to 2015) of the canadian health measures survey," 2017.

[4] S. Smith and J. Bernstein, "Therapeutic uses of lung aerosols," Lung biology in health and disease, vol. 94, pp. 233-269, 1996.

[5] S. Fart and G. Taylor, "Insulin inhalation: its potential as a nonparenteral method of administration," Lung biology in health and disease, vol. 107, pp. 371-388, 1997.

[6] K. G. Schüepp, D. Straub, A. Möller, and J. H. Wildhaber, "Deposition of aerosols in infants and children," Journal of aerosol medicine, vol. 17, no. 2, pp. 153-156, 2004.

[7] B. M. Crosland, "An experimental characterisation of the velocities of the spray issued from a pressurised metered-dose inhaler," Master's thesis, 2008.

[8] J. A. Pongracic, "Asthma delivery devices: age-appropriate use.," Pediatric annals, vol. 32, no. 1, p. 50, 2003. 
[9] H. Versteeg and G. Hargrave, "Near-orifice spray and valve flow regime of a pharmaceutical pressurised metered dose inhaler," Zaragoza, vol. 9, p. 11, 2002.

[10] H. Versteeg, G. Hargrave, and M. Kirby, "Internal flow and near-orifice spray visualisations of a model pharmaceutical pressurised metered dose inhaler," in Journal of Physics: Conference Series, vol. 45, p. 207, IOP Publishing, 2006.

[11] G. Wigley, H. Versteeg, and D. Hodson, "Near-orifice pda measurements and atomisation mechanism of a pharmaceutical pressurised metered dose inhaler," Zaragoza, vol. 9, p. 11, 2002.

[12] C. Dunbar, An experimental and theoretical investigation of the spray issued from a pressurised metered-dose inhaler. PhD thesis, University of Manchester, 1996.

[13] C. Dunbar and J. Miller, "Theoretical investigation of the spray from a pressurized metered-dose inhaler," Atomization and Sprays, vol. 7, no. 4, 1997.

[14] B. M. Crosland, M. R. Johnson, and E. A. Matida, "Characterization of the spray velocities from a pressurized metered-dose inhaler," Journal of aerosol medicine and pulmonary drug delivery, vol. 22, no. 2, pp. 85-98, 2009.

[15] H. K. Versteeg, G. K. Hargrave, B. Myatt, D. Lewis, T. Church, and G. Brambilla, "Using phase doppler anemometry \& high speed imaging to analyze mdi spray plume dynamics," 2017.

[16] C. Dunbar, A. Watkins, and J. Miller, "An experimental investigation of the spray issued from a pmdi using laser diagnostic techniques," Journal of aerosol medicine, vol. 10, no. 4, pp. 351-368, 1997.

[17] X. Liu, W. H. Doub, and C. Guo, "Evaluation of metered dose inhaler spray velocities using phase doppler anemometry (pda)," International journal of pharmaceutics, vol. 423, no. 2, pp. 235-239, 2012. 
[18] C. Terzano, "Pressurized metered dose inhalers and add-on devices," Pulmonary pharmacology $\&$ therapeutics, vol. 14, no. 5, pp. 351-366, 2001.

[19] S. P. Newman, "Principles of metered-dose inhaler design," Respiratory care, vol. 50, no. 9, pp. 1177-1190, 2005.

[20] W. Finlay, K. Stapleton, and P. Zuberbuhler, "Errors in regional lung deposition predictions of nebulized salbutamol sulphate due to neglect or partial inclusion of hygroscopic effects," International journal of pharmaceutics, vol. 149, no. 1, pp. 63-72, 1997.

[21] S. P. Newman, "Aerosol deposition considerations in inhalation therapy," Chest Journal, vol. 88, no. 2_Supplement, pp. 152S-160S, 1985.

[22] B. K. Rubin and J. B. Fink, "Optimizing aerosol delivery by pressurized metereddose inhalers," Respiratory care, vol. 50, no. 9, pp. 1191-1200, 2005.

[23] G. Crompton, "Problems patients have using pressurized aerosol inhalers.," European journal of respiratory diseases. Supplement, vol. 119, p. 101, 1982.

[24] B. K. Rubin and J. B. Fink, "The delivery of inhaled medication to the young child," Pediatric Clinics of North America, vol. 50, no. 3, pp. 717-731, 2003.

[25] A. Kamps, P. Brand, and R. Roorda, "Determinants of correct inhalation technique in children attending a hospital-based asthma clinic," Acta Paediatrica, vol. 91, no. 2, pp. 159-163, 2002.

[26] F. Lavorini, C. Corrigan, P. Barnes, P. Dekhuijzen, M. Levy, S. Pedersen, N. Roche, W. Vincken, and G. Crompton, "Retail sales of inhalation devices in european countries: so much for a global policy," Respiratory medicine, vol. 105, no. 7, pp. 1099-1103, 2011. 
[27] D. Price, J. Marshall, and R. Turner, "Pmd92 inhaler use in five european countries: Analysis of sales data from q4 2005 to q4 2011," Value in Health, vol. 15, no. 7, p. A361, 2012.

[28] B. K. Rubin, "What does it mean when a patient says, "my asthma medication isn't working?," Chest Journal, vol. 126, no. 3, pp. 972-981, 2004.

[29] N. Roche and P. R. Dekhuijzen, "The evolution of pressurized metered-dose inhalers from early to modern devices," Journal of aerosol medicine and pulmonary drug delivery, vol. 29, no. 4, pp. 311-327, 2016.

[30] W. Finlay, "Chap. 8," The Mechanics of Inhaled Pharmaceutical Aerosols: An Introduction, Academic Press, London, 2001.

[31] B. Rubin, M. Newhouse, and P. Barnes, "Conquering childhood asthma. hamilton (canada)," 1998.

[32] P. Barry and C. o'Callaghan, "Multiple actuations of salbutamol mdi into a spacer device reduce the amount of drug recovered in the respirable range," European Respiratory Journal, vol. 7, no. 9, pp. 1707-1709, 1994.

[33] D. Clark and B. Lipworth, "Effect of multiple actuations, delayed inhalation and antistatic treatment on the lung bioavailability of salbutamol via a spacer device.," Thorax, vol. 51, no. 10, pp. 981-984, 1996.

[34] J. Berry, S. Heimbecher, J. L. Hart, and J. Sequeira, "Influence of the metering chamber volume and actuator design on the aerodynamic particle size of a metered dose inhaler," Drug development and industrial pharmacy, vol. 29, no. 8, pp. 865876, 2003.

[35] M. Mellon, J. Leflein, K. Walton-Bowen, M. Cruz-Rivera, S. Fitzpatrick, and J. A. Smith, "Comparable efficacy of administration with face mask or mouthpiece of 
nebulized budesonide inhalation suspension for infants and young children with persistent asthma," American journal of respiratory and critical care medicine, vol. 162, no. 2, pp. 593-598, 2000.

[36] F. Lavorini, "The challenge of delivering therapeutic aerosols to asthma patients," ISRN allergy, vol. 2013, 2013.

[37] S. W. Stein and C. G. Thiel, "The history of therapeutic aerosols: a chronological review," Journal of aerosol medicine and pulmonary drug delivery, vol. 30, no. 1, pp. 20-41, 2017.

[38] P. W. Longest and M. Hindle, "Evaluation of the respimat soft mist inhaler using a concurrent cfd and in vitro approach," Journal of aerosol medicine and pulmonary drug delivery, vol. 22, no. 2, pp. 99-112, 2009.

[39] P. Rogliani, L. Calzetta, A. Coppola, F. Cavalli, J. Ora, E. Puxeddu, M. G. Matera, and M. Cazzola, "Optimizing drug delivery in copd: the role of inhaler devices," Respiratory medicine, vol. 124, pp. 6-14, 2017.

[40] R. J. Schick, "Spray technology reference guide: Understanding drop size," Spraying Systems Co. Bulletin B, vol. 459, pp. 8-16, 2006.

[41] A. Gosman and E. Ioannides, "Aspects of computer simulation of liquid-fueled combustors. aiaa 19th aerospace sciences meeting," tech. rep., AIAA-81-0323, St. Louis, Missouri, 1981.

[42] B. Launder and D. Spalding, "Lectures in mathematical models of turbulent flows," 1972.

[43] P. P. Kakade, H. K. Versteeg, G. K. Hargrave, P. Genova, R. C. Williams Iii, and D. Deaton, "Design optimization of a novel pmdi actuator for systemic drug delivery," Journal of Aerosol Medicine, vol. 20, no. 4, pp. 460-474, 2007. 
[44] H. Smyth, G. Brace, T. Barbour, J. Gallion, J. Grove, and A. J. Hickey, "Spray pattern analysis for metered dose inhalers: effect of actuator design," Pharmaceutical research, vol. 23, no. 7, pp. 1591-1596, 2006.

[45] G. Pitcairn, S. Reader, D. Pavia, and S. Newman, "Deposition of corticosteroid aerosol in the human lung by respimat soft mist inhaler compared to deposition by metered dose inhaler or by turbuhaler dry powder inhaler," Journal of aerosol medicine, vol. 18, no. 3, pp. 264-272, 2005.

[46] P. Anderson, "Use of respimat soft mist inhaler in copd patients," International journal of chronic obstructive pulmonary disease, vol. 1, no. 3, p. 251, 2006.

[47] H. Wachtel and J. Ziegler, "Improved assessment of inhaler device performance using laser diffraction," Respiratory Drug Delivery VIII, vol. 2, pp. 379-381, 2002.

[48] M. Spallek, D. Hochrainer, and H. Wachtel, "Optimizing nozzles for soft mist inhalers," in Respiratory Drug Delivery, vol. 8, pp. 375-378, 2002.

[49] D. Hochrainer, H. Hölz, C. Kreher, L. Scaffidi, M. Spallek, and H. Wachtel, "Comparison of the aerosol velocity and spray duration of respimat soft mist inhaler and pressurized metered dose inhalers," Journal of aerosol medicine, vol. 18, no. 3, pp. 273-282, 2005.

[50] A. Heenan, W. Finlay, B. Grgic, A. Pollard, and P. Burnell, "An investigation of the relationship between the flow field and regional deposition in realistic extrathoracic airways," Journal of aerosol science, vol. 35, no. 8, pp. 1013-1023, 2004.

[51] Á. Farkas, I. Balásházy, and K. Szőcs, "Characterization of regional and local deposition of inhaled aerosol drugs in the respiratory system by computational fluid and particle dynamics methods," Journal of aerosol medicine, vol. 19, no. 3, pp. 329-343, 2006. 
[52] H. Jin, J. Fan, M. Zeng, and K. Cen, "Large eddy simulation of inhaled particle deposition within the human upper respiratory tract," Journal of aerosol Science, vol. 38, no. 3, pp. 257-268, 2007.

[53] H. Takano, N. Nishida, M. Itoh, N. Hyo, and Y. Majima, "Inhaled particle deposition in unsteady-state respiratory flow at a numerically constructed model of the human larynx," Journal of aerosol medicine, vol. 19, no. 3, pp. 314-328, 2006.

[54] A. Heenan, E. Matida, A. Pollard, and W. Finlay, "Experimental measurements and computational modeling of the flow field in an idealized human oropharynx," Experiments in Fluids, vol. 35, no. 1, pp. 70-84, 2003.

[55] C. Kleinstreuer, H. Shi, and Z. Zhang, "Computational analyses of a pressurized metered dose inhaler and a new drug-aerosol targeting methodology," Journal of Aerosol Medicine, vol. 20, no. 3, pp. 294-309, 2007.

[56] P. W. Longest, M. Hindle, and S. D. Choudhuri, "Effects of generation time on spray aerosol transport and deposition in models of the mouth-throat geometry," Journal of aerosol medicine and pulmonary drug delivery, vol. 22, no. 2, pp. 67-84, 2009.

[57] G. Alhegagi, "Pressurized metered-dose inhaler(pMDI) aerosol deposition measurements in add-on spacers," in Masters Abstracts International, vol. 48, 2009.

[58] R. F. Oliveira, S. Teixeira, L. F. Silva, J. C. Teixeira, and H. Antunes, "Study of a pressurized metered-dose inhaler spray parameters in fluent ${ }^{T M}$," in Proceedings of the World Congress on Engineering, vol. 2, 2010.

[59] N. Ogrodnik, V. Azzi, E. Sprigge, S. Fiset, and E. Matida, "Nonuniform deposition of pressurized metered-dose aerosol in spacer devices," Journal of aerosol medicine and pulmonary drug delivery, vol. 29, no. 6, pp. 490-500, 2016. 
[60] A. J. Hickey, "Methods of aerosol particle size characterization," Pharmaceutical Inhalation Aerosol Technology, ed, vol. 2, pp. 345-384, 2004.

[61] C. Davies, "Particle-fluid interaction," Journal of Aerosol Science, vol. 10, no. 5, pp. 477-513, 1979.

[62] T. F. Hatch, P. Gross, et al., "Pulmonary deposition and retention of inhaled aerosols.," Pulmonary Deposition and Retention of Inhaled Aerosols., 1964.

[63] S. Alaboud, In-vitro inhalation performance for formoterol dry powder and metred dose inhalers. In-vitro characteristics of the emitted dose from the formoterol dry powder and metred dose inhalers to identify the influence of inhalation flow, inhalation volume and the number of inhalation per dose. $\mathrm{PhD}$ thesis, University of Bradford, 2013.

[64] J. P. Seville and C.-Y. Wu, Particle Technology and Engineering: An Engineer's Guide to Particles and Powders: Fundamentals and Computational Approaches. Butterworth-Heinemann, 2016.

[65] A. Davis, A fundamental study of the flow and droplet delivery from a pressurised metered dose inhaler (pMDI). PhD thesis, (C) AJ Davis, 2008.

[66] N. Labiris and M. Dolovich, "Pulmonary drug delivery. part i: physiological factors affecting therapeutic effectiveness of aerosolized medications," British journal of clinical pharmacology, vol. 56, no. 6, pp. 588-599, 2003.

[67] Chaker, Mustapha and Meher-Homji, Cyrus B and Mee, Thomas, "title=Inlet Fogging of Gas Turbine Engines: Part A- Fog Droplet Thermodynamics, Heat Transfer and Practical Considerations," in ASME Turbo Expo 2002: Power for Land, Sea, and Air, pp. 413-428, American Society of Mechanical Engineers, 2002. 
[68] R. W. Wilmott, T. F. Boat, A. Bush, V. Chernick, R. R. Deterding, and F. Ratjen, Kendig and Chernick's Disorders of the Respiratory Tract in Children E-Book. Elsevier Health Sciences, 2012.

[69] D. S. Gardenhire, Rau's Respiratory Care Pharmacology-E-Book. Elsevier Health Sciences, 2015.

[70] J. Kenney, "Moving averages," Mathematics of Statistics, vol. 1, pp. 221-223, 1962.

[71] E. Whittaker and G. Robinson, "Graduation, or the smoothing of data," The Calculus of Observations: A Treatise on Numerical Mathematics, 4th Ed. Dover, New York, pp. 285-316, 1967.

[72] H.-E. Albrecht, N. Damaschke, M. Borys, and C. Tropea, Laser Doppler and phase Doppler measurement techniques. Springer Science \& Business Media, 2003.

[73] J. A. Kleppe, J. G. Olin, and R. K. Menon, "29.1 pitot probe anemometry," 1999.

[74] T. J. Callahan, T. Ryan III, L. Dodge, and J. Schwalb, "Effects of fuel properties on diesel spray characteristics," tech. rep., Southwest Research Institute, San Antonio, TX, 1987.

[75] S. Ristić, "Laser doppler anemometry and its application in wind tunnel tests," Scientific Technical Review, vol. 57, no. 3-4, pp. 64-76, 2007.

[76] C. Tu, Z. Yin, J. Lin, and F. Bao, "A review of experimental techniques for measuring micro-to nano-particle-laden gas flows," Applied Sciences, vol. 7, no. 2, p. $120,2017$.

[77] D. Dynamics, "Phase Doppler Anemometry (PDA) - PDA HiDense Spray System," Sept. 2002. 
[78] F. Mayinger and O. Feldmann, "Optical measurements: techniques and applications," Optical Measurements: Techniques and Applications, vol. 1, 2001.

[79] S. G. Daviault, "Characterization of the fuel injection process within the ignition quality tester (iqt)," Master's thesis, Carleton University, 2011.

[80] Y. Yeh and H. Cummins, "Localized fluid flow measurements with an hene laser spectrometer," Applied Physics Letters, vol. 4, no. 10, pp. 176-178, 1964.

[81] D. Dynamics, "Particle dynamics analysis- Measurement principles of PDA," Jan. 2001.

[82] J. A. Lock and G. Gouesbet, "Generalized lorenz-mie theory and applications," Journal of Quantitative Spectroscopy and Radiative Transfer, vol. 110, no. 11, pp. 800-807, 2009.

[83] D. Dynamics, BSA flow software installation and user's guides, 2006.

[84] E. Matida and G. Alhegagi, "Numerical simulation of pmdi aerosol deposition in add-on spacers," in American Association for Aerosol Research 29th Annual Conference, Portland, Oregon, 2010.

[85] I. Brezani and F. Zelenak, "Improving the effectivity of work with rosin-rammler diagram by using matlab (r) gui tool," Acta Montanistica Slovaca, vol. 15, no. 2, pp. 152-157, 2010.

[86] H. Versteeg and W. Malalasekera, "An introduction to computional fluid dynamics: The finite volume method," 2007.

[87] F. R. Menter, "Two-equation eddy-viscosity turbulence models for engineering applications," AIAA journal, vol. 32, no. 8, pp. 1598-1605, 1994. 
[88] O. Gryczka, S. Heinrich, N. Deen, M. van Sint Annaland, J. Kuipers, M. Jacob, and L. Mörl, "Characterization and cfd-modeling of the hydrodynamics of a prismatic spouted bed apparatus," Chemical engineering science, vol. 64, no. 14, pp. 3352-3375, 2009.

[89] E. A. Matida, W. H. Finlay, M. Breuer, and C. F. Lange, "Improving prediction of aerosol deposition in an idealized mouth using large-eddy simulation," Journal of aerosol medicine, vol. 19, no. 3, pp. 290-300, 2006.

[90] C. Kleinstreuer, "Modern fluid dynamics: Basic theory and selected applications in macro- and micro-fluidics," in Modern Fluid Dynamics, vol. 87, Springer, 2010.

[91] T. Hayase, J. Humphrey, and R. Greif, "A consistently formulated quick scheme for fast and stable convergence using finite-volume iterative calculation procedures," Journal of Computational Physics, vol. 98, no. 1, pp. 108-118, 1992.

[92] P. W. Longest and S. Vinchurkar, "Effects of mesh style and grid convergence on particle deposition in bifurcating airway models with comparisons to experimental data," Medical Engineering and Physics, vol. 29, no. 3, pp. 350-366, 2007.

[93] J. H. Ferziger and M. PERIĆ, "Further discussion of numerical errors in cfd," International Journal for Numerical Methods in Fluids, vol. 23, no. 12, pp. 1263$1274,1996$.

[94] A. Agresti and B. Finlay, Logistic Regression-Modeling Categorical Responses. Statistical Methods for Social Sciences. Upper Saddle River, Prentice Hall, 1997.

[95] R. F. Oliveira, S. Teixeira, J. C. Teixeira, L. F. Silva, and H. Antunes, "pmdi sprays: theory, experiment and numerical simulation," in Advances in Modeling of Fluid Dynamics, InTech, 2012. 
[96] M. S. Khan, M. S. Awan, S. S. Muhammad, M. Faisal, F. NADEEM, E. LEITGEB, et al., "Probabilistic model for free-space optical links under continental fog conditions.," Radioengineering, vol. 19, no. 3, 2010.

[97] C. A. Dunbar and A. J. Hickey, "Evaluation of probability density functions to approximate particle size distributions of representative pharmaceutical aerosols," Journal of aerosol science, vol. 31, no. 7, pp. 813-831, 2000.

[98] R. S. Figliola and D. Beasley, Theory and design for mechanical measurements. John Wiley \& Sons, 2015.

[99] J. Ziegler and H. Wachtel, "Comparison of cascade impaction and laser diffraction for particle size distribution measurements," Journal of aerosol medicine, vol. 18, no. 3, pp. 311-324, 2005.

[100] A. R. Martin and W. H. Finlay, "The effect of humidity on the size of particles delivered from metered-dose inhalers," Aerosol science and technology, vol. 39, no. 4, pp. 283-289, 2005.

[101] C. Dunbar and J. Mitchell, "Analysis of cascade impactor mass distributions," Journal of aerosol medicine, vol. 18, no. 4, pp. 439-451, 2005.

[102] S. P. Newman, J. Brown, K. P. Steed, S. J. Reader, and H. Kladders, "Lung deposition of fenoterol and flunisolide delivered using a novel device for inhaled medicines: comparison of respimat with conventional metered-dose inhalers with and without spacer devices," Chest Journal, vol. 113, no. 4, pp. 957-963, 1998.

[103] S. Bell, "A beginner's guide to uncertainty of measurement," Measurement good practice guide, vol. 11, p. 1, 1999.

[104] R. Abernethy, R. Benedict, and R. Dowdell, "Asme measurement uncertainty," Journal of Fluids Engineering, vol. 107, no. 2, pp. 161-164, 1985. 
[105] S. Tavoularis, Measurement in fluid mechanics. Cambridge University Press, 2005.

[106] S. Nichols, J. Mitchell, C. Shelton, and D. Roberts, "Good cascade impactor practice (gcip) and considerations for "in-use" specifications," Aaps Pharmscitech, vol. 14, no. 1, pp. 375-390, 2013.

[107] R. Kapulla and S. B. Najera, "Operation conditions of a phase doppler anemometer: droplet size measurements with laser beam power, photomultiplier voltage, signal gain and signal-to-noise ratio as parameters," Measurement Science and Technology, vol. 17, no. 1, p. 221, 2005.

[108] B. J. O'Connor, "The ideal inhaler: design and characteristics to improve outcomes," Respiratory medicine, vol. 98, pp. S10-S16, 2004.

[109] A. Alatrash and E. Matida, "Characterization of medication velocity and size distribution from pressurized metered-dose inhalers by phase doppler anemometry," Journal of aerosol medicine and pulmonary drug delivery, vol. 29, no. 6, pp. 501-513, 2016. 


\section{Chapter 7}

\section{Uncertainty in measurments}

\subsection{Errors in measurements for PDA system}

Table 7.1: Measurement errors for particle size using PDA system for tested inhalers $75(\mathrm{~mm})$ at $95 \%$ level of confidence.

\begin{tabular}{|l|c|c|c|c|c|}
\hline Inhaler type & $\begin{array}{c}\text { Independent } \\
\text { samples } \%\end{array}$ & $\begin{array}{c}\mathrm{SD} \\
\text { value } \sigma_{\bar{d}} \\
(\mu \mathrm{m})\end{array}$ & $\begin{array}{c}\text { Bias } \\
\text { limit } \\
(\mu \mathrm{m})\end{array}$ & $\begin{array}{c}\text { Precision } \\
\text { limit } \\
(\mu \mathrm{m})\end{array}$ & $\begin{array}{c}\text { Uncertainty } \\
( \pm \mu m)\end{array}$ \\
\hline Apo-Salvent CFC Free & 47.48 & 0.08 & 0.02 & 0.03 & 0.03 \\
\hline Airomir HFA & 43.15 & 0.06 & 0.02 & 0.02 & 0.03 \\
\hline Novo-Salbutamol HFA & 46.89 & 0.08 & 0.02 & 0.03 & 0.03 \\
\hline Ventolin HFA & 46.93 & 0.06 & 0.02 & 0.02 & 0.03 \\
\hline
\end{tabular}


Table 7.2: Measurement errors for particle size using PDA system for tested inhalers $100(\mathrm{~mm})$ at $95 \%$ level of confidence.

\begin{tabular}{|l|c|c|c|c|c|}
\hline Inhaler type & $\begin{array}{c}\text { Independent } \\
\text { samples } \%\end{array}$ & $\begin{array}{c}\text { SD } \\
\text { value } \sigma_{\bar{d}} \\
(\mu m)\end{array}$ & $\begin{array}{c}\text { Bias } \\
\text { limit } \\
(\mu m)\end{array}$ & $\begin{array}{c}\text { Precision } \\
\text { limit } \\
(\mu m)\end{array}$ & $\begin{array}{c}\text { Uncertainty } \\
( \pm \mu m)\end{array}$ \\
\hline Apo-Salvent CFC Free & 44.17 & 0.08 & 0.02 & 0.02 & 0.03 \\
\hline Airomir HFA & 45.14 & 0.07 & 0.02 & 0.02 & 0.03 \\
\hline Novo-Salbutamol HFA & 40.76 & 0.08 & 0.02 & 0.03 & 0.04 \\
\hline Ventolin HFA & 48.61 & 0.05 & 0.02 & 0.01 & 0.02 \\
\hline
\end{tabular}

Table 7.3: Measurement errors for particle velocity using PDA system for tested inhalers $75(\mathrm{~mm})$ at $95 \%$ level of confidence.

\begin{tabular}{|l|c|c|c|c|c|}
\hline Inhaler type & $\begin{array}{c}\text { Independent } \\
\text { samples } \%\end{array}$ & $\begin{array}{c}\text { SD } \\
\text { value } \\
\sigma_{\bar{d}}(\mathrm{~m} / \mathrm{s})\end{array}$ & $\begin{array}{c}\text { Bias } \\
\text { limit } \\
(\mathrm{m} / \mathrm{s})\end{array}$ & $\begin{array}{c}\text { Precision } \\
\text { limit } \\
(\mathrm{m} / \mathrm{s})\end{array}$ & $\begin{array}{c}\text { Uncertainty } \\
( \pm \mathrm{m} / \mathrm{s})\end{array}$ \\
\hline Apo-Salvent CFC Free & 47.48 & 0.16 & 0.02 & 0.11 & 0.11 \\
\hline Airomir HFA & 43.15 & 0.15 & 0.02 & 0.10 & 0.10 \\
\hline Novo-Salbutamol HFA & 46.89 & 0.13 & 0.02 & 0.08 & 0.08 \\
\hline Ventolin HFA & 46.93 & 0.17 & 0.02 & 0.13 & 0.13 \\
\hline
\end{tabular}


Table 7.4: Measurement errors for particle velocity using PDA system for tested inhalers $100(\mathrm{~mm})$ at $95 \%$ level of confidence.

\begin{tabular}{|l|c|c|c|c|c|}
\hline Inhaler type & $\begin{array}{c}\text { Independent } \\
\text { samples } \%\end{array}$ & $\begin{array}{c}\text { SD } \\
\text { value } \\
\sigma_{\bar{d}}(\mathrm{~m} / \mathrm{s})\end{array}$ & $\begin{array}{c}\text { Bias } \\
\text { limit } \\
(\mathrm{m} / \mathrm{s})\end{array}$ & $\begin{array}{c}\text { Precision } \\
\text { limit } \\
(\mathrm{m} / \mathrm{s})\end{array}$ & $\begin{array}{c}\text { Uncertainty } \\
( \pm \mathrm{m} / \mathrm{s})\end{array}$ \\
\hline Apo-Salvent CFC Free & 44.17 & 0.16 & 0.02 & 0.12 & 0.12 \\
\hline Airomir HFA & 45.14 & 0.18 & 0.02 & 0.15 & 0.15 \\
\hline Novo-Salbutamol HFA & 40.76 & 0.16 & 0.02 & 0.11 & 0.11 \\
\hline Ventolin HFA & 48.61 & 0.17 & 0.02 & 0.13 & 0.13 \\
\hline
\end{tabular}

Table 7.5: Measurement errors for particle diameter using PDA system for tested Spiriva Respimat inhaler at $95 \%$ level of confidence.

\begin{tabular}{|l|c|c|c|c|c|}
\hline $\begin{array}{l}\text { Location } \\
(\mathrm{mm})\end{array}$ & Independent & SD & Bias & Precision & $\begin{array}{c}\text { Uncertainty } \\
( \pm \mu m)\end{array}$ \\
\hline-18.5 & 71.50 & 0.01 & 0.02 & 0.00 & 0.02 \\
\hline 0 & 90.96 & 0.01 & 0.02 & 0.00 & 0.02 \\
\hline 75 & 66.67 & 0.03 & 0.02 & 0.00 & 0.02 \\
\hline 100 & 98.54 & 0.02 & 0.02 & 0.00 & 0.02 \\
\hline
\end{tabular}


Table 7.6: Measurement errors for particle velocity using PDA system for tested Spiriva Respimat inhaler at $95 \%$ level of confidence.

\begin{tabular}{|l|c|c|c|c|c|}
\hline $\begin{array}{l}\text { Location } \\
(\mathrm{mm})\end{array}$ & $\begin{array}{c}\text { Independent } \\
\text { samples } \%\end{array}$ & $\begin{array}{c}\text { SD } \\
\text { value } \\
\sigma_{\bar{d}}(\mathrm{~m} / \mathrm{s})\end{array}$ & $\begin{array}{c}\text { Bias } \\
\text { limit } \\
(\mathrm{m} / \mathrm{s})\end{array}$ & $\begin{array}{c}\text { Precision } \\
\text { limit } \\
(\mathrm{m} / \mathrm{s})\end{array}$ & $\begin{array}{c}\text { Uncertainty } \\
( \pm \mathrm{m} / \mathrm{s})\end{array}$ \\
\hline-18.5 & 71.88 & 0.06 & 0.02 & 0.02 & 0.03 \\
\hline 0 & 76.29 & 0.03 & 0.02 & 0.00 & 0.02 \\
\hline 75 & 78.84 & 0.05 & 0.02 & 0.01 & 0.02 \\
\hline 100 & 77.29 & 0.07 & 0.02 & 0.02 & 0.03 \\
\hline
\end{tabular}




\subsection{Errors in measurements for particle size and de- position using ACI}

Table 7.7: Measurement errors for particle size using ACI system under $>90 \% \mathrm{RH}$ at $95 \%$ level of confidence.

\begin{tabular}{|l|c|c|c|}
\hline Stage & $\begin{array}{c}\text { Bias } \\
\text { limit } \\
(\mu m)\end{array}$ & $\begin{array}{c}\text { Precision } \\
\text { limit }(\mu m)\end{array}$ & $\begin{array}{c}\text { Uncertainty } \\
( \pm \mu m)\end{array}$ \\
\hline 0 & 0.02 & 0.00 & 0.027 \\
\hline 1 & 0.02 & 0.00 & 0.02 \\
\hline 2 & 0.02 & 0.00 & 0.02 \\
\hline 3 & 0.03 & 0.00 & 0.03 \\
\hline 4 & 0.05 & 0.00 & 0.05 \\
\hline 5 & 0.09 & 0.00 & 0.09 \\
\hline 6 & 0.07 & 0.00 & 0.07 \\
\hline 7 & 0.07 & 0.00 & 0.07 \\
\hline
\end{tabular}


Table 7.8: Measurement errors for deposition on ACI system under $>90 \% \mathrm{RH}$ at $95 \%$ level of confidence.

\begin{tabular}{|l|c|c|c|}
\hline Stage & Bias & $\begin{array}{c}\text { Precision } \\
\text { limit } \%\end{array}$ & $\begin{array}{c}\text { Uncertainty } \\
\%\end{array}$ \\
\hline IP & 0.01 & 0.00 & 0.01 \\
\hline 0 & 0.02 & 0.00 & 0.02 \\
\hline 1 & 0.02 & 0.00 & 0.02 \\
\hline 2 & 0.02 & 0.00 & 0.02 \\
\hline 3 & 0.03 & 0.00 & 0.03 \\
\hline 4 & 0.05 & 0.00 & 0.05 \\
\hline 5 & 0.09 & 0.00 & 0.09 \\
\hline 6 & 0.07 & 0.00 & 0.07 \\
\hline 7 & 0.07 & 0.00 & 0.07 \\
\hline
\end{tabular}

